\title{
Elucidating and Pharmacologically Targeting Secondary Injury Cascades following Neural Injury
}

Brandon P. Lucke-Wold

Follow this and additional works at: https://researchrepository.wvu.edu/etd

\section{Recommended Citation}

Lucke-Wold, Brandon P., "Elucidating and Pharmacologically Targeting Secondary Injury Cascades following Neural Injury" (2016). Graduate Theses, Dissertations, and Problem Reports. 6120.

https://researchrepository.wvu.edu/etd/6120

This Dissertation is protected by copyright and/or related rights. It has been brought to you by the The Research Repository @ WVU with permission from the rights-holder(s). You are free to use this Dissertation in any way that is permitted by the copyright and related rights legislation that applies to your use. For other uses you must obtain permission from the rights-holder(s) directly, unless additional rights are indicated by a Creative Commons license in the record and/ or on the work itself. This Dissertation has been accepted for inclusion in WVU Graduate Theses, Dissertations, and Problem Reports collection by an authorized administrator of The Research Repository @ WVU.

For more information, please contact researchrepository@mail.wvu.edu. 


\section{Elucidating and Pharmacologically Targeting Secondary Injury Cascades following}

\section{Neural Injury}

Brandon P. Lucke-Wold

Dissertation submitted

to the School of Medicine

at West Virginia University

in partial fulfillment of the requirements for the degree of

Doctor of Philosophy in

Neuroscience

Charles L. Rosen, M.D., Ph.D., Chair

Jason D. Huber, Ph.D.

James P. O’Callaghan, Ph.D.

Rae R. Matsumoto, Ph.D.

James Bibb, Ph.D.

Department of Neurosurgery

The Center for Neuroscience

Morgantown, West Virginia

2016

Keywords: chronic traumatic encephalopathy, blast traumatic brain injury, endoplasmic reticulum stress, protein kinase $\mathrm{C}$ activity, oxidative stress

Copyright 2016 Brandon P. Lucke-Wold 


\title{
ABSTRACT \\ Elucidating and Pharmacologically Targeting Secondary Injury Cascades following Neural Injury
}

Brandon P. Lucke-Wold

\begin{abstract}
3.4 million concussions occur each year in the United States. Recent evidence suggests that some of these individuals are susceptible to neurodegenerative disease development following traumatic brain injury. The unknown factor is how acute injury contributes to this degenerative process. A prominent neurotrauma related neurodegenerative disease is chronic traumatic encephalopathy (CTE). CTE is defined by neurofibrillary tau tangles with a perivascular distribution and mood disturbances. In order to elucidate the pathologic changes associated with CTE, it is imperative to utilize adequate preclinical models. We have strategically developed and tested a clinically relevant rodent blast model. The model reliably produces a CTE phenotype including tauopathy, cell death, impulsivity, and cognitive decline. Using this validated model, we investigated several important secondary injury cascades that link acute brain injury to chronic neurodegenerative changes. More importantly, we pharmacologically targeted these pathways and found improved pathologic and behavioral outcomes. In chapter 1, we discuss the potential mechanisms linking acute injury to CTE in athletes and soldiers. In chapter 2, we highlight the physics behind the compression wave produced by our model and how this wave produces injury. In chapter 3, data is presented regarding the CTE phenotype generated by our model following repetitive blast exposure in rodents. Chapter 4 focuses on the role of blood brain barrier disruption and how targeting protein kinase $\mathrm{C}$ activity with bryostatin reduces this disruption. In chapter 5 , we look at the role endoplasmic reticulum stress plays in human pathologic specimens from patients diagnosed with CTE and in rodents following repeat blast. We found that docosahexaenoic acid successfully targeted endoplasmic reticulum stress, reduced tauopathy, and improved cognitive performance. In chapter 6, we looked at lipoic acid and its role in reducing NADPH oxidative stress following repetitive neurotrauma. We found that lipoic acid reduces impulsive-like behavior and decreased cell death. Finally, in chapter 7 we discuss important strategies for improving preclinical models going forward and what needs to be investigated to improve our understanding of CTE. In this dissertation, we highlight important secondary injury cascades including blood brain barrier disruption, protein kinase $\mathrm{C}$ activity, endoplasmic reticulum stress, and oxidative stress that warrant further investigation for the development of novel treatment approaches for CTE.
\end{abstract}


This work is dedicated to my loving and supportive family

Noelle, Antony, Cleo, Lynn, Kevin, Bob, Debra, Michelle, and Trevor 


\section{ACKNOWLEDGEMENTS}

The work generated during my $\mathrm{PhD}$ years could not have been possible without the guidance and support of multiple individuals.

Dr. Turner, thank you for your never-ending mentorship and guidance through the whole process. You continue to provide excellent advice and friendship as we advance through our training.

Aric, Drs. Robson, Bonasso, and Nguyen, thank you for working so closely on the multiple projects. I have thoroughly enjoyed the collaboration. I wish you all the utmost success in your future careers. I look forward to continued collaboration as colleagues.

Drs. Tan, Li, Regele, He, Alkon, Bailes, and Lee, thank you for your individual guidance and expertise on the projects we collaborated on.

Drs. Miller and O'Callaghan, thank you for your guidance on techniques, the use of NIOSH equipment, and advice on science. Diane, thank you for supervising the sleep and TBI review.

Drs. Huber and Dey, thank you for writing all of the recommendation letters. Dr. Huber I appreciated your expert guidance on the blood brain barrier and neuropharmacology. Dr. Dey, thank you for your direction of the Neurobiology and Anatomy Department.

Penny and Mary Anne thank you for your constant support, helping with paperwork, and keeping us on track. We would not have been able to do it without you.

Drs. Matsumoto and Bibb, thank you for your guidance and edits on our papers and helping provide expert advice.

Dr. Ciporen, thank you for allowing me to come work at OHSU for an internship. I learned a great deal from the anatomic simulation studies and appreciated your life and career advice. I look forward to further projects together.

Dr. Rosen, thank you for being there for me throughout the entire process. Your door was always open to discuss projects, talk about life, and provide guidance for career training. You allowed us to pursue projects of interest and supported our intellectual curiosity. Thank you for the multiple hours you spent reviewing papers, grants, and writing recommendation letters. I look forward to the next stage as I transition to clinical work.

Noelle, thank you for being my better half for these past 10 years that we have known each other. You are my biggest support and will always encourage me to be a better man and become the best clinician scientist possible. None of it would be possible without you by my side.

I would also like to thank my funders: the American Association of Pharmaceutical Education Pre-doctoral grant, WVU Distinguished Doctoral Grant, American Medical Association Foundation Seed Grant, Sigma Xi Grants in Aid of Research, and Neurosurgery Research and Education Medical Student Summer Research Fellowship. 


\section{TABLE OF CONTENTS}

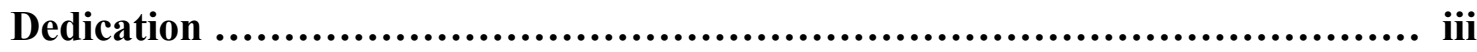

Acknowledgements............................................................... iv

Table of Contents.................................................................... v

List of Figures....................................................................... vii

List of Tables....................................................................... ix

List of Abbreviations............................................................ $x$

Chapter 1: Linking traumatic brain injury to chronic traumatic encephalopathy: Identification of potential mechanisms leading to neurofibrillary tangle development

Abstract............................................................................ 02

Introduction.................................................................... 03

Neuropatholical Findings in CTE................................................. 04

Background on Tau.................................................................. 08

Discussion........................................................................... 14

Clinical Relevance and Conclusions............................................... 17

Acknowledgements................................................................ 18

Chapter 2: Elucidating the role of compression waves and impact duration for generating mild traumatic brain injury in rats

Abstract................................................................................. 26

Introduction....................................................................... 27

Methods........................................................................ 28

Results.......................................................................... 33

Discussion........................................................................ 38

Acknowledgements................................................................ 40

Chapter 3: The quest to model chronic traumatic encephalopathy: A multiple model \& injury paradigm experience

Abstract................................................................................. 50

Introduction........................................................................ 51

Methods............................................................................ 53

Results................................................................................... 58

Discussion..................................................................... 65

Acknowledgements.......................................................... 68

Chapter 4: Bryostatin-1 Restores Blood Brain Barrier Integrity following Blast-Induced Traumatic Brain Injury

Abstract............................................................................. 82 
Introduction............................................................................ 83

Methods.............................................................................. 85

Results............................................................................ 92

Discussion....................................................................... 97

Acknowledgements............................................................... 101

Chapter 5: Endoplasmic reticulum stress implicated in chronic traumatic encephalopathy

Abstract.......................................................................... 120

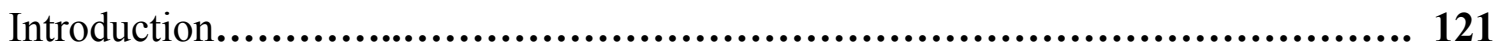

Methods.................................................................................... 123

Results.................................................................................. 130

Discussion.............................................................................. 137

Conclusion........................................................................ 140

Disclosures and Acknowledgements................................................. 141

Chapter 6: Amelioration of NADPH-mediated stress reduces cell death following blastinduced traumatic brain injury

Abstract............................................................................ 163

Introduction........................................................................ 164

Materials and Methods........................................................... 167

Results............................................................................ 173

Discussion............................................................................ 181

Conclusion.............................................................................. 186

Acknowledgements............................................................... 187

Chapter 7: Modeling chronic traumatic encephalopathy: The way forward for future discovery

Abstract........................................................................... 208

Introduction...................................................................... 209

The Quest for the Ideal CTE Model................................................... 211

Implications for Modeling CTE: Results from Prior CTE Modeling Studies.......... 214

Implications for Modeling CTE: Learning from Past Shortcomings with TBI Models 218

Key Questions Going Forward..................................................... 220

Summary................................................................................ 239

Acknowledgements................................................................ 240

Chapter 8: Final Discussion

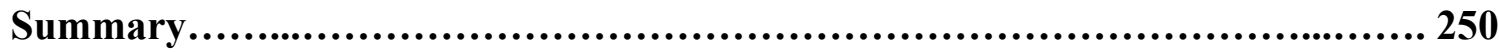

Future Directions.................................................................. 252

References.......................................................................... 254 


\section{INDEX OF FIGURES}

page

\section{Chapter 1}

1.1 Traumatic brain injury leading to tauopathy.................................. 19

1.2 Tau phosphorylation sites....................................................... 20

1.3 Neurofibrillary tangle formation................................................ 21

\section{Chapter 2}

2.1 Geometry of sensor layout..................................................... 42

2.2 Contour plot 2D pressure................................................... 43

2.3 L2 error........................................................................... 44

2.4 x-t diagram...................................................................... 45

2.5 Pressure vs. time by sensor........................................................ 46

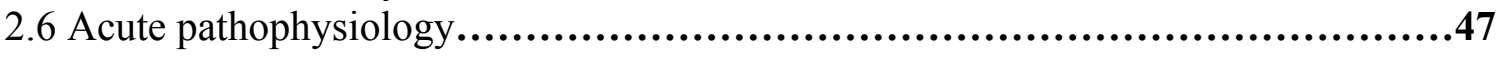

2.7 Neuronal degeneration.............................................................. 48

\section{Chapter 3}

3.1 Experimental schematic............................................................. 69

3.2 Schematic of the models.......................................................... 70

3.3 Increased tau hyperphosphorylation............................................... 71

3.4 Tau hyperphosphorylation in superficial cortex.................................. 73

3.5 Tau conformational change..................................................... 74

3.6 Tau conformation change in superficial cortex.................................... 76

3.7 Perivascular tau................................................................. 77

3.8 Learning and memory deficits post-blast........................................... 78

3.9 Morris water maze track plots...................................................... 79

3.10 Blast increases impulsive-like behavior....................................... 80

\section{Chapter 4}

4.1 Schematic of experimental techniques........................................... 103

4.2 Bryostatin-1 targets PKC isozymes............................................... 104

4.3 Bryostatin-1 is a potent PKC modulator................................................106

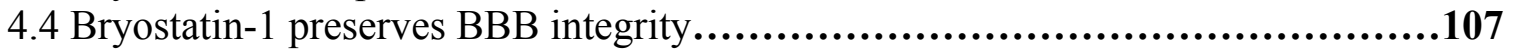

4.5 Vascular disruption after blast exposure..........................................108

4.6 Bryostatin-1 regulates tight junction proteins......................................109

4.7 Tight junction proteins modulated near vasculature................................ 111

4.8 PKC activity prominent in endothelial cells..................................... 112

$4.9 \mathrm{PKC} \delta$ in endothelial cells not astrocytes........................................ 114

4.10 PKC $\varepsilon$ in endothelial cells not astrocytes.......................................... 116

Supplementary figure 4.1 Von Willebrand Factor and $\mathrm{PKC} \alpha . . . . . . . . . . . . . . . . . . . . . . .118$ 


\section{Chapter 5}

5.1 Robust pathologic changes seen after repetitive blast............................ 143

5.2 Human CTE tauopathy........................................................ 145

5.3 ER stress following blast injury............................................. 147

5.4 Tauopathy and ER stress........................................................ 149

5.5 CHOP is increased following blast.............................................. 151

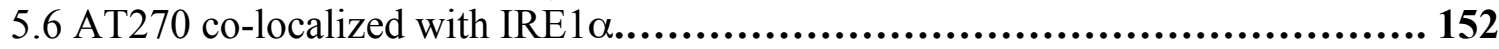

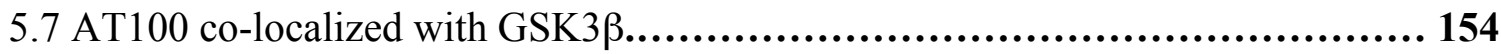

5.8 DHA reduces markers of ER stress.......................................... 156

5.9 ER stress increases tau kinases................................................ 157

5.10 DHA prevents cognitive decline.............................................. 158

Supplementary figure 5.1 Blast schematic........................................ 160

Supplementary figure 5.2 Track plots............................................ 161

\section{Chapter 6}

6.1 NADPH oxidative stress increased following blast................................. 189

6.2 Oxidative stress increased post blast.............................................. 191

6.3 Lipoic acid decreases oxidative stress biproducts................................. 192

6.4 Blast decreases Bcl-2 and HO-1 ............................................... 193

6.5 Pro-apoptotic markers acutely post blast........................................... 195

$6.6 \mathrm{HO}-1$ and Bcl-2 2 weeks post blast............................................... 196

6.7 Pro-apoptotic markers 2 weeks post blast.......................................... 197

6.8 NADPH increased in CTE samples................................................ 199

6.9 Pro-apoptotic markers in CTE samples........................................... 200

6.10 Lipoic acid reduces tau conformational changes................................. 202

6.11 Tauopathy in human CTE brains.............................................. 204

6.12 Lipic acid decreases impulsive-like behavior.................................. 205

Supplementary figure 6.1 Pathway schematic........................................ 206

\section{Chapter 7}

7.1 Methodological challenges of TBI models......................................... 241

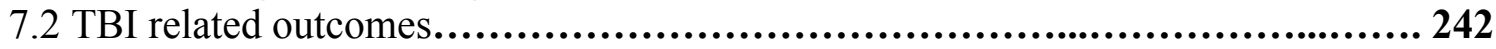




\section{INDEX OF TABLES}

page

Table 1.1 List of tau kinases and function........................................... 24

Table 7.1 Collection of studies for CTE............................................... 243

Table 7.2 Collection of studies for blast-induced neurotrauma......................... 244

Table 7.3 Collection of studies using weight drop model............................... 245

Table 7.4 Collection of studies using controlled cortical impact........................ 246

Table 7.5 Collection of studies using fluid percussion.................................... 247

Table 7.6 Less conventional models of neurotrauma.................................. 248

Table 7.7 In vivo and ex vivo TBI models............................................. 249 


\section{LIST OF ABBREVIATIONS}

A- $\mathrm{A} \beta=$ amyloid Beta, $\mathrm{AD}=\mathrm{Alzheimer}$ 's disease, $\mathrm{AMP} A=\alpha$-amino-3-hydroxy-5-methyl4-isoxazolepropionic acid, ANOVA=one-way analysis of variance, $\mathrm{APO} \varepsilon 4=$ apolipoprotein $\varepsilon 4, \mathrm{APP}=$ amyloid precursor protein, $\mathrm{ATF} 6=$ activating transcription factor 6

B- $\mathrm{BBB}=$ blood brain barrier, $\mathrm{BCA}=$ bicinchoninic acid, $\mathrm{BiP}=$ binding immunoglobulin protein, $\mathrm{Bryo}=$ bryostatin, $\mathrm{bTBI}=$ blast traumatic brain injury

C- $\mathrm{CDK} 5=$ cycline-dependent kinase $5, \mathrm{CFL}=$ Courant-Freidrich-Lewis, $\mathrm{CHOP}=\mathrm{C} / \mathrm{EBP}$ homologus protein, $\mathrm{CNS}=$ central nervous system, $\mathrm{CTE}=$ chronic traumatic encephalopathy, $\mathrm{CTEM}=$ chronic traumatic encephalomyelopathy, $\mathrm{CTRL}=$ control

D- $\mathrm{DAB}=$ diaminobenzadine, $\mathrm{DHA}=$ docosahexaenoic acid, DPBS=Dulbecco's phosphate buffered saline, dTAI=diffuse traumatic axonal injury

E- EB=Evan's blue, eIF2 $\alpha=$ eukaryotic initiation factor 2 alpha, $E R=$ endoplasmic reticulum, ERK1/2=extracellular signal-regulated kinases

F- FJB=fluorojade B, FST=forced swim test

G- GADD34-growth arrest and DNA damage-inducible protein, GFAP=glia fibrillary acidic protein, GSK3 $\beta$, glycogen synthase kinase 3 Beta, $G T=$ glial tangles

H- H\&E=hematoxylin and eosin, $\mathrm{HO}-1=$ heme oxygenase 1

I- IHC=immunohistochemisty, i.p. $=$ intraperitoneal, IRE $1 \alpha=$ inositol requiring enzyme 1 alpha

J- JNK=c-Jun N-terminal Kinase

L- LA=alpha lipoic acid, LTP=long term potentiation

M- mapt $=$ microtubule associated protein tau, MRI=magnetic resonance imaging, MRS=magenetic resonance spectroscopy, $\mathrm{MWM}=$ Morris water maze

$\mathbf{N}$ - NADPH=nicotinamide adenine dinucleotide phosphate, $\mathrm{NFL}=$ national football league, NFTs $=$ neurofibrillary tangles, $\mathrm{NMDA}=\mathrm{N}$-methyl-D-aspartate, $\mathrm{Nox}=\mathrm{NADPH}$ oxidase, NSE=neuron-specific enolase

P- PERK=protein kinase-like ER kinase, PET=positron emission tomography, $\mathrm{PHF}=$ paired helical filament, $\mathrm{PKA}=$ protein kinase $\mathrm{A}, \mathrm{PKB}=$ protein kinase $\mathrm{B}$, $\mathrm{PKC}=$ protein kinase $\mathrm{C}, \mathrm{PSI}=$ pounds per square inch

$\mathbf{R}-\mathrm{RB}=$ repeat blast, $\mathrm{ROS}=$ reactive oxygen species

$\mathrm{S}-\mathrm{SB}=$ single blast, $\mathrm{SD}=$ Sprague Dawley, $\mathrm{SDS}=$ sodium dodecyl sulfate, $\mathrm{SGK}=$ serum and glucocorticoid-regulated kinase, SOD1=superoxide dismutase 
$\mathbf{T}-\mathrm{TBI}=$ traumatic brain injury, $\mathrm{TCA}=$ trichloroacetic acid, $\mathrm{TDP} 43=$ transactive response DNA binding protein $43, \mathrm{TLRs}=$ toll-like receptors

V- VWF $=$ Von Willebrand Factor

W- WWE=world wrestling entertainment

$\mathrm{X}-\mathrm{XBP} 1=\mathrm{X}-$ box binding protein 1

Z- ZO-1=zona occludens 1 


\section{Chapter One}

Linking traumatic brain injury to chronic traumatic encephalopathy: Identification of potential mechanisms leading to neurofibrillary tangle development

This work is published in J Neurotrauma. 2014 Jul 1;31(13):1129-38. doi: 10.1089/neu.2013.3303. Epub 2014 Apr 11. Review. PMID: 24499307

Lucke-Wold BP, Turner RC, Logsdon AF, Bailes JE, Huber JD, Rosen CL 


\section{ABSTRACT}

Significant attention has recently been drawn to the potential link between head trauma and the development of neurodegenerative disease, namely chronic traumatic encephalopathy (CTE). The acute neurotrauma associated with sports related concussions in athletes and blast induced traumatic brain injury in soldiers elevates the risk for future development of chronic neurodegenerative diseases such as CTE. CTE is a progressive disease distinguished by characteristic tau neurofibrillary tangles (NFTs) and occasionally transactive response DNA binding protein 43 (TDP43) oligomers; both of which have a predilection for perivascular and subcortical areas near reactive astrocytes and microglia. The disease is currently only diagnosed post-mortem by neuropathologic identification of NFTs. A recent workshop sponsored by National Institute of Neurological Disorders and Stroke emphasized the need for pre-mortem diagnosis to better understand disease pathophysiology and to develop targeted treatments. In order to accomplish this objective, it is necessary to discover the mechanistic link between acute neurotrauma and the development of chronic neurodegenerative and neuropsychiatric disorders such as CTE. In this review we briefly summarize what is currently known about CTE development and pathophysiology, and subsequently discuss injury-induced pathways that warrant further investigation. Understanding the mechanistic link between acute brain injury and chronic neurodegeneration will facilitate the development of appropriate diagnostic and therapeutic options for CTE and other related disorders.

KEY WORDS: chronic traumatic encephalopathy, neurofibrillary tangles 


\section{INTRODUCTION}

Neurotrauma is one of the most common injuries in contact sports and military conflicts (Goldstein et al 2012). Each year in the United States alone, over 1.7 million traumatic brain injuries (TBIs) occur (Gavett et al 2011b). Many of these TBIs are related to participation in contact sports, such as football and hockey, but a high rate of neurotrauma has also been reported for civilian and military populations in war zones (Stern et al 2011). In fact, the US Department of Defense labeled blast induced TBI (bTBI) as the "signature injury" of the recent wars in Iraq and Afghanistan (Goldstein et al 2012). The estimated annual cost of treatment for bTBI in the United States is 2.5 billion dollars (Shenton et al 2012). The enormous economic burden is due, in part, to the progressive development of cognitive, motor, and psychiatric problems in blast-exposed veterans and civilians (Peskind et al 2013). These clinical symptoms, emerging in former athletes and soldiers alike, are often the first measurable signs for the development of a chronic neurodegenerative disease such as chronic traumatic encephalopathy (CTE) (Stern et al 2011). Clinical presentation of CTE has recently been divided into two categories: young age of onset with primarily psychiatric and behavioral problems, and old age of onset with primarily cognitive and motor deficits (Stern et al 2013). Increased awareness about CTE has prompted widespread investigation into the progression and pathophysiology of this disease (McKee et al 2009).

The two populations at greatest risk for development of CTE are professional athletes and soldiers (Peskind et al 2013). It appears that individuals with one or two copies of the apolipoprotein $\varepsilon 4(A P O \varepsilon 4)$ allele have poorer outcome following head trauma and are at increased risk for developing CTE following TBI (Spillantini et al 
2011). Athletes exposed to subconcussive and concussive injury, as well as soldiers exposed to even a single blast, can develop behavioral and psychiatric problems within a single year following injury (Bailes et al 2013). An area in need of further investigation is how acute neurotrauma relates to and/or causes chronic neurodegenerative diseases in susceptible individuals. In this review, we examine tau-based CTE pathophysiology and disease progression while discussing potential mechanistic pathways that may link acute neurotrauma with chronic neurodegenerative disease development and NFT formation.

\section{NEUROPATHOLOGICAL FINDINGS IN CTE}

Post-mortem examination is currently the only widely-utilized and accepted method by which CTE is diagnosed clinically, although in vivo approaches have been identified and are currently under development for diagnosis and tracking pre-mortem (Small et al 2013, Stern et al 2011). Common neuropathologic findings of CTE include NFTS and transactive response DNA binding protein 43 (TDP43), as well as microglial and astrocyte activation (Lakis et al 2013). Although the mechanistic link responsible for these pathological outcomes is not fully known, the current understanding of the pathologic progression will be discussed in the following paragraphs (Stern et al 2013).

$\underline{N F T s}$

Normal tau binds to tubulin and stabilizes microtubule fibrils in neurons, thereby facilitating neurite outgrowth. When tau becomes hyperphosphorylated it binds to other normal tau proteins, which leads to aggregation (Iqbal et al 2013). Tau hyperphosphorylation in the central nervous system (CNS) is common after TBI and other brain injuries (Mannix et al 2013). TBI can cause normal tau to dissociate from 
tubulin; thereby, exposing multiple phosphorylation sites (Walker \& Tesco 2013). Hyperphosphorylated tau is no longer able to bind to tubulin and translocates from the axon to the neuron soma (Iqbal et al 2013). A primary reason for this translocation is that normal tau is soluble while hyperphosphorylated tau becomes insoluble thus favoring a paired helical filament arrangement that is too large to function in axons (Mannix et al 2013). The paired helical arrangement also leads to poor clearance of hyperphosphorylated tau from the neuron (Walker \& Tesco 2013). Accumulation of insoluble tau within neurons contributes to the development of tau oligomers (Cowan \& Mudher 2013). Tau oligomers are granular intracellular buildups of mutated tau, which precede the development of NFTs (Walker \& Tesco 2013). When tau phosphatases can no longer dephosphorylate oligomers efficiently, NFTs grow and eventually mature (Whittington et al 2013). NFT maturation involves the acetylation of the lysine residue 280 (Cowan \& Mudher 2013). Once NFTs fully mature, they affect large projecting neurons in a progressive hierarchical pattern (de Calignon et al 2012). NFTs can spread to surrounding at-risk neurons through trans-synaptic propagation or extracellular secretion as depicted in figure 1.1 (Le et al 2012). Propagation can occur by direct seeding of tau oligomers into the lipid rafts of cell membranes thus increasing cell permeability and allowing access for the spread of larger secreted NFTs (Whittington et al 2013). After NFTs propagate, the post-translational modifications become finalized and behavioral and motor symptoms begin to surface in patients (Walker \& Tesco 2013). A likely reason for the symptomatic changes is that NFTs cause neurons to become deinnervated, in part, due to decreased neurite outgrowth, which ultimately leads to neuronal death (Cowan \& Mudher 2013). 
Wild-type TDP43 is a nuclear RNA/DNA binding protein that regulates the transcription of thousands of genes (Hebron et al 2013). TDP43 is predominantly found in large motor neurons throughout the CNS (Bandyopadhyay et al 2013). TBI causes an up-regulation of $\mathrm{Ca}^{2+}$-permeable $\alpha$-amino-3-hydroxy-5-methyl-4-isoxazolepropionic acid (AMPA) receptors, which in turn lead to carboxy-terminal-cleaved TDP43 fragments (Yamashita et al 2012). These fragments translocate to the cytosol mediated in part by the process of ubiquitination (Hebron et al 2013). The fragments form intracellular aggregates that are representative of several neurodegenerative diseases including: Alzheimer's disease, Parkinson's disease, amyotrophic lateral sclerosis, and CTE (Bandyopadhyay et al 2013). Aggregates sequester RNA leading to pronounced neurotoxicity (Yamashita et al 2012). One mechanism by which neurotoxicity occurs is TDP43 aggregate induced misfolding of $\mathrm{Cu} / \mathrm{Zn}$ superoxide dismutase (SOD1), which predisposes surrounding cells to free-radical damage (Bandyopadhyay et al 2013). Associated clinical symptoms of TDP43 pathology include cognitive and motor impairment (Yamashita et al 2012). Cognitive impairment may take the form of apathy, poor impulse control, and lack of overall executive judgment (McKee et al 2010). It has yet to be determined exactly how TDP43 aggregates coincide and interact with NFTs to produce the wide spectrum of clinical CTE presentation seen in patients (Le et al 2012).

\section{Astrocyte activation}

Astrocytes in a healthy brain provide a supporting role for neurons (Sirko et al 2013). If brain injury occurs, astrocytes become responsive by increasing expression of a 
microfilament known as glia fibrillary acidic protein (GFAP), while simultaneously releasing cytokines that activate nearby neurons to increase nociceptive receptivity (Wang et al 2009). Astrocytes also trigger self-renewing neurospheres that may help in brain recovery (Sirko et al 2013). bTBI, in particular, causes increased astrocyte activation and GFAP levels within 24 hours post-injury (Kochanek et al 2013). Peripheral GFAP is absorbed and sequestered from the plasma immediately following bTBI leading to an initial decrease in serum levels at 6 hours, but GFAP is subsequently increased by augmented gene expression and changes in membrane permeability at 24 hours (Arun et al 2013a). The process of astrocyte activation involves an increase in astrocyte size, number, and motility that primarily occurs in the white matter following brain or spinal injury (Wang et al 2009). The activation is most pronounced in the corpus callosum, motor, and somatosensory cortex leading to symptoms of increased impulsive behavior and cognitive dysfunction (Kochanek et al 2013). One mechanism by which astrocytes are activated following brain injury is microglia-mediated crosstalk via pro-inflammatory cytokines (Wang et al 2009). These immune cells release interleukin-1 $\beta$ and other cytokines that act on toll-like receptors (TLRs) in astrocytes; thereby, inducing astrocytes to release reactive oxygen species (ROS) (Ferreira et al 2013). ROS indirectly cause excitotoxicity by decreasing the ability of astrocytes to uptake glutamate (Qureshi et al 2004). This excitotoxicity was shown to cause changes in exploratory behavior and distinct motor deficits in mice exposed to cortical impact TBI (Madathil et al 2013).

\section{Microglial activation}

Microglia are ramified immune cells of the brain that are inactive in healthy brains (Lai et al 2013). Following repetitive TBI, localized cell death activates microglia 
in the striatum and thalamus, which can be measured by the markers OX6 and CD68 (Acosta et al 2013). Activated microglia have a bushy appearance with thickened processes and enlarged cell bodies (Lai et al 2013). Furthermore, the microglia foster the spread of neuroinflammation (Hernandez-Ontiveros et al 2013). Short-term activation of microglia is neuroprotective, while chronic activation is involved in neurodegeneration (Acosta et al 2013). Chronic activation becomes more common in an aged brain accounting in part for the progressive nature of neurodegenerative diseases (Kumar et al 2013). Glial tangles (GT), for example, are prominent in the frontal and temporal lobes of CTE brains many years after initial injury (Stern et al 2011). A primary reason for the persistence of microglia activation is due to diffuse traumatic axonal injury (dTAI) (Smith 2013). dTAI can cause the up-regulation of surface antigens on microglia that ultimately triggers the release of inflammatory cytokines (Hernandez-Ontiveros et al 2013). Sensorimotor behavioral deficits following dTAI have been reported, possibly as a result of microglia induced inflammatory tissue damage (Smith 2013).

\section{BACKGROUND ON TAU}

\section{$\underline{\text { Tau isoforms and tau mutations }}$}

The gene responsible for encoding tau is microtubule-associated protein tau (mapt) on chromosome 17 (Rossi et al 2014). After tau is encoded, six isoforms can form by various splicing of exons 2,3 , or 10 on the microtubule binding domain of pre-mRNA (Avale et al 2013). The exon 10 splice-variant, hTau40, is the most important in neurodegenerative disease development and is found specifically in the central nervous system (Agarwal et al 2013). The isoforms consist of three (3R) or four (4R) tau repeats 
inserted at the carboxyl terminus (Takuma et al 2003). Ideally, the ratio between 3R and 4R isoforms in the brain is maintained at 1:1 (Sadik et al 2009b). When mutations occur in the exon 10 splice-variant, the ratio is shifted to favor an increase in the $3 \mathrm{R}$ isoforms (Agarwal et al 2013). Normally, apolipoprotein E in the brain helps catalyze the proteolytic breakdown of mutated tau and restore the ideal 3R to 4R ratio, but the APO 44 allele produces an apolipoprotein that is ineffective in this reaction (Stern et al 2011). When mutations persist, they play an important role in NFT development and the activation of astrocytes (Spillantini et al 2011). The altered tau proteins have widespread pathologic consequences (Le et al 2012).

\section{$\underline{\text { Tau kinase overview }}$}

Tau has 79 potential binding sites, and phosphorylation of these sites plays an important role in embryonic CNS development (Kanemaru et al 1992). 30 functional sites on the normal tau protein can be phosphorylated in the adult brain as depicted in figure 1.2 , but the amount of phosphorylation is kept to a minimum by tau phosphatases (Billingsley \& Kincaid 1997). In the adult brain, tau is regulated through multi-site phosphorylation at serine/threonine residues by proline-kinases such as extracellular signal-regulated kinases (ERK1/2), cycline-dependent kinase 5 (CDK5), and glycogen synthase kinase 3- $\beta$ (GSK3- $\beta$ ) (Tsujio et al 2000). Other non proline-kinases such as protein kinase C (PKC), c-Jun kinase (JNK), Akt, and various tyrosine kinases play a secondary role in tau phosphorylation (Zeng et al 2010). Following TBI, tau hyperphosphorylation is increased due to an elevation in kinases compared to phosphatases as depicted in figure 1.3, marking an initial pathologic change that indicates future development of chronic neurodegeneration (Liu et al 2013). The key tau kinases 
are described in Table 1.1 and discussed in further detail with relation to changes caused by TBI in the following paragraphs.

\section{$\underline{E R K 1 / 2}$}

ERK1/2 is dephosphorylated following a single mild TBI (Kuo et al 2013). The dephosphorylated ERK1/2 triggers apoptosis via caspase3 activation (Zhao et al 2012). Administration of estrone after TBI triggers ERK1/2 phosphorylation and pro-survival (Gatson et al 2012). This hormone warrants further investigation in regards to its potential role in ameliorating tau hyperphosphorylation. Estrone may initiate a neuroprotective priming response that protects against subsequent injury (Gatson et al 2012). Other kinases, such as p70S6K and mitogen and stress activated protein kinase 1, may also be involved in this neuroprotective process (Chen et al 2007, Li et al 2013b). Facilitation of ERK1/2 via the compound PD90859 can additionally foster cell survival after brain injury (Mori et al 2002). Besides mediating the complex balance between cell survival and apoptosis, ERK1/2 plays another unique role by regulating the cytoskeleton of activated astrocytes following TBI (Kramerov et al 2011). ERK1/2 is therefore important not only for tau hyperphosphorylation but also in the process of reactive astrogliosis.

\section{$\underline{C D K 5}$}

Following TBI, CDK5 acutely binds to its receptor and activates a pro-apoptotic cascade (Li et al 2013a). Furthermore, CDK5 triggers cell-cycle activation and microglia activation following controlled cortical impact TBI (Kabadi et al 2012a). The extent and duration of microglia activation mediated by CDK5 requires further investigation. It is 
known that inhibition of CDK5 with the roscovitine derivative, CR8, promotes neuroprotection and decreased apoptosis after controlled cortical impact (Kabadi et al 2012b). Additionally, roscovitine itself can improve cognitive and motor function in Sprague Dawley rats after TBI (Hilton et al 2008). CDK5 inhibitors even improve outcome when administered several hours to days after TBI (Johnson et al 2010a). How these inhibitors alter the progression of tauopathies has yet to be investigated.

\section{$\underline{G S K 3-\beta}$}

Protein kinase B (PKB) and serum and glucocorticoid-regulated kinase (SGK) are activated following TBI, which both subsequently phosphorylate GSK3- $\beta$ (Shapira et al 2007, Wu et al 2013). GSK3- $\beta$ activation via its phosphorylation has been linked to apoptosis and tau hyperphosphorylation (Wang et al 2013b). Furthermore, GSK3- $\beta$ upregulates NMDA receptors following brain injury causing an exacerbation of glutamate excitotoxicity (Deng et al 2014). Inhibition of GSK3- $\beta$ consequently reduces apoptosis and the extent of excitotoxicity (Dash et al 2011). Humanin is a potential inhibitor of GSK3- $\beta$ that increases neuroprotection following brain injury, but further studies are still needed to elucidate the mechanism of action (Wang et al 2013c). GSK3- $\beta$ is also functionally significant in microglial migration, translocation of monocytes across the BBB, and inflammatory cascades following TBI (Lyman et al 2014). GSK3- $\beta$ may therefore be a key target in discovering the link between acute brain injury and chronic neurodegeneration due to its primary roles in both microglia migration and tau hyperphosphorylation.

\section{$\underline{P K C}$}


The five most common isoforms of $\operatorname{PKC}(\alpha, \delta, \varepsilon, \zeta, \eta)$ play various supporting roles as Ser/Thr kinases throughout multiple tissues in the body (Kim et al 2003). Activation of specific PKC isoforms $(\alpha, \delta$, and $\zeta)$ is associated with perturbations in tight junction proteins following brain injury, which ultimately leads to increased BBB permeability (Combs et al 1998). The disruption in the BBB further increases PKC activity thereby triggering the tau kinase, GSK3- $\beta$ (Martin et al 2013). PKC $\eta$ additionally activates the pro-survival tau kinase, Akt, at several days post-injury (Cho 2011). After hyperphosphorylation occurs, PKC $\alpha$ maintains the phosphorylation changes by inhibiting tau phosphatases (Combs et al 1998). PKC prompts signal cascades that work in conjunction with altered calcium homeostasis to propel the development of NFTs (Martin et al 2013). Since PKC involvement is intimately associated with tau hyperphosphorylation and NFT formation, it seems reasonable to investigate the role of selective PKC inhibitors/activators, such as bryostatin and balonol, in the prevention of chronic tauopathies like CTE (Zohar et al 2011).

$\underline{J N K}$

JNK is increased in damaged axons following TBI (Tran et al 2012). JNK activity is also markedly increased in neurons and astrocytes of the hippocampus following TBI (Otani et al 2002). JNK signaling can cause post-traumatic cellular damage within the brain following injury (Tran et al 2012). JNK additionally may phosphorylate p53, which enhances neuronal autophagy (Hong et al 2012). When JNK is inhibited, the extent of abnormal tau hyperphospharylation is lessened (Tran et al 2012). Furthermore, glucagon has been used to inhibit JNK signaling immediately after TBI causing a decrease in intracranial cerebrovasodilation (Armstead et al 2012). Maintaining JNK signaling within 
a tightly controlled range is not only important for tau regulation but also for maintaining blood flow to the brain following TBI (Armstead et al 2011).

$\underline{A k t}$

Akt produces an interesting effect following brain injury by phosphorylating tau at Ser212, but also inhibiting GSK3- $\beta$ (Ksiezak-Reding et al 2003). GSK3- $\beta$ activation may therefore be necessary for initial tau hyperphosphorylation, but Akt activity maintains hyperphosphorylation at later time points (Zeng et al 2010). By inhibiting GSK3- $\beta$, it is thought that Akt triggers an anti-apoptotic pathway allowing for damaged cells to survive and propogate NFTs (Ksiezak-Reding et al 2003). If Akt is inhibited, cell death will occur (Farook et al 2013). Histone deacetylase inhibitors, such as Scriptaid, prevent the dephosphorylation of Akt, and therefore increase the number of surviving neurons after TBI (Wang et al 2013a). Akt regulation warrants further investigation to tease out the level of activation that is necessary for maintaining neuroprotective properties while avoiding the spread of NFTs.

\section{Tau phosphatases}

Two phosphatases, protein phosphatase 1 and 2A, are responsible for maintaining tau in a non-hyperphosphorylated state (Billingsley \& Kincaid 1997). If these two phosphatases become dysfunctional or decreased, the hyperphosphorylated tau is quickly ubiquitinated, which then predisposes the neuron to increased NFT formation (Lambrecht et al 2013). TBI can cause a decrease in tau phosphatases (Kane et al 2012). In particular, protein phosphatase $2 \mathrm{~A}$ is decreased in the hippocampus for several weeks post-TBI, which results in dysfunctional hippocampus plasticity (Bales et al 2010). Tau 
phosphatase activity must drop by half before NFTs will begin to develop (Lambrecht et al 2013). Compounds that increase tau phosphatases, such as sodium selenate, may prove promising in slowing the progression of tauopathies (Corcoran et al 2010).

\section{DISCUSSION}

Although the post-mortem pathology of CTE has been well described, the mechanism by which acute TBI leads to initial tau hyperphosphorylation and the eventual development of neurofibrillary tangles remains poorly understood. Since the term CTE was only recently reintroduced into the medical literature in 2005 , understanding disease pathophysiology is in its infancy (Omalu et al 2005). Despite the link between TBI and CTE being associational rather than mechanistic at this point, the growing prevalence of this disease amongst soldiers, football players, wrestlers, and other athletes exposed to head injuries increases the urgency for finding a causative mechanism, and furthermore locating pharmacologic targets for treating this devastating disease. In the following paragraphs, we discuss a few molecular pathways previously associated with other forms of brain injury that warrant further investigation following TBI.

\section{Endoplasmic reticulum stress}

The endoplasmic reticulum (ER) is responsible for the correct folding and sorting of proteins (Rubovitch et al 2011). Following brain injury, the ER becomes dysfunctional, as evidenced by changes in bound intracellular calcium, leading to the accumulation of unfolded proteins within the cell (Osada et al 2009). The increase in unfolded proteins is known as the ER stress response (Cullinan \& Diehl 2006). Ischemic stroke and TBI can both cause acute activation of the ER stress response (Srinivasan \& 
Sharma 2012). Three arms of the ER stress response (protein kinase-like ER kinase (PERK), inositol requiring enzyme $1 \alpha$ (IRE1 $\alpha$ ), and activating transcription factor 6 (ATF6)) regulate the amount of pro-apoptotic activity following injury (Rubovitch et al 2011). All three arms affect the protein expression of C/EBP-homologous protein (CHOP) (Osada et al 2009). CHOP is noteworthy for its ability to trigger apoptosis via the activation of caspase12 (Srinivasan \& Sharma 2012). If CHOP is maintained belowthreshold by the PERK arm, neuronal apoptosis does not occur (Cullinan \& Diehl 2006). When CHOP is pushed beyond threshold through activation of the IRE1 $\alpha$ and ATF6 arms, neuronal apoptosis does occur, and tau hyperphosphorylation results from GSK3- $\beta$ (Hoozemans et al 2009). Furthermore, two downstream targets of the ATF6 arm of the ER stress pathway are MAPK and JNK, which may subsequently be involved in tau hyperphosphorylation as well (Krajewska et al 2011). In light of these findings, it may prove beneficial to utilize a pharmacologic agent that attenuates the ER stress response in a model of TBI. Salubrinal can increase activity of the neuroprotective PERK arm of the ER stress response and inhibit the pro-apoptotic activity of the IRE1 $\alpha$ arm of the ER stress response (Gong et al 2012). Since GSK3- $\beta$ and caspase12 are increased following brain injury, it may also be worth investigating the GSK3- $\beta$ peptide inhibitors, L803-mts and TDZD-8, and the role they may play in preventing the development of NFTs (Hoozemans et al 2009, King et al 2014).

\section{Glutamate excitotoxicity}

Glutamate excitotoxicity is triggered following brain injury and results in elevated intracellular calcium, formation of reactive oxygen species, and mitochondrial failure (Wagner \& Zitelli 2013). Ischemia and other forms of brain injury can cause an increase 
in calcium that activates $\alpha$-calcium/calmodulin protein kinase II, leading to memory impairment via increased AMPA receptor activity in the hippocampus (Atkins et al 2006). The increased calcium also leads to intracellular accumulation in neuronal mitochondria, making the organelle dysfunctional (Wagner \& Zitelli 2013). Activated microglia and astrocytes concurrently release interleukin-6, which triggers a further increase in intracellular calcium within neurons and sensitizes N-Methyl D-aspartate (NMDA) receptors (Ma et al 2013). Sensitized NMDA receptors promote auxiliary excitotoxicity and foster the release of reactive oxygen species from the mitochondria, which can eventually cause neuronal destruction (Qureshi et al 2004). Caspase3, a proapoptotic factor, is increased following glutamate induced mitochondria dysfunction (Srinivasan \& Sharma 2012). Caspase3 can cause tau cleavage and predisposes the neuron to NFT development (Hoozemans et al 2009). To stem the tide of neuronal destruction and progressive tau changes, it seems befitting to investigate compounds that are known to decrease the amount of reactive oxygen species such as the NADPHoxidase inhibitor, apocynin (Ferreira et al 2013). Furthermore, targeting mitochondrial dysfunction through p38 inhibitors may also prove beneficial following head trauma (Huang et al 2013). By targeting key downstream pathways of glutamate excitotoxicity, it may be possible to alleviate the potential progression to neurodegeneration.

\section{Microglial and astrocyte regulators}

Neurotrauma can result in a dynamic equilibrium between classically activated (M1) and alternatively activated (M2) microglia (Acosta et al 2013). M1 microglia are pro-inflammatory while M2 microglia are anti-inflammatory (Smith 2013). Targeting the activation of M2 microglia immediately following injury may prove beneficial in 
preventing neurodegeneration (Kuo et al 2008). Alternatively, acutely inhibiting M1 microglia with the noncompetitive cholinesterase inhibitor, donepezil, has also decreased neuroinflammation and apoptosis after TBI (Hwang et al 2010). Similarly, neurotrauma triggers two distinct responses, pro-survival or apoptosis, in activated astrocytes depending on the extent and duration of injury (Kochanek et al 2013). If astrocyte activation extends several days post-injury, it was found that nitration of tau occurs, which may lead to a more rapid development of NFTs (Reyes et al 2008). Furthermore, mutations in tau may be occurring in activated astrocytes, resulting in tau oligomers being subsequently secreted into the extracellular milieu (de Calignon et al 2012). Future studies are needed to characterize the time-course of astrocyte activation following TBI, and more importantly at what point it is ideal to inhibit astrocyte activation.

\section{CLINICAL RELEVANCE AND CONCLUSIONS}

The Veterans Affairs Healthcare System reported that patients exposed to repetitive blast waves have quantitative electroencephalogram changes that are comparable to concussive injury (Tompkins et al 2013). Similarly, the detection of repetitive concussions in athletes has increased significantly over the past 20 years (Small et al 2013). The duration between injuries may account for why certain individuals develop rapidly progressive neurodegeneration and increased phospho-tau expression (Mannix et al 2013). The Department of Defense has recently invested 700 million dollars into improving clinical diagnosis and care for the $266,810 \mathrm{bTBI}$ patients who were injured from 2001 onwards (Tompkins et al 2013). Likewise, the National Football League has recently organized a new medical committee to investigate the issue of TBI, and started a multi-prong approach for making football safer for the players (Ellenbogen 
et al 2010). Increased investigation into understanding the pathology of CTE will hopefully aid in pre-mortem diagnosis as well as finding viable treatment options. The pathways mentioned in this review (ER Stress, glutamate excitotoxicty, and microglia and astrocyte modulation) appear promising in understanding the link between acute bTBI and CTE development. Discovering the process of tau hypephosphorylation and NFT development following TBI will likely provide a key for unlocking the unknown mysteries of similar progressive neurodegenerative diseases.

\section{ACKNOWLEDGEMENTS}

We would like to acknowledge Ann Noelle Lucke-Wold for her help in editing this manuscript. 


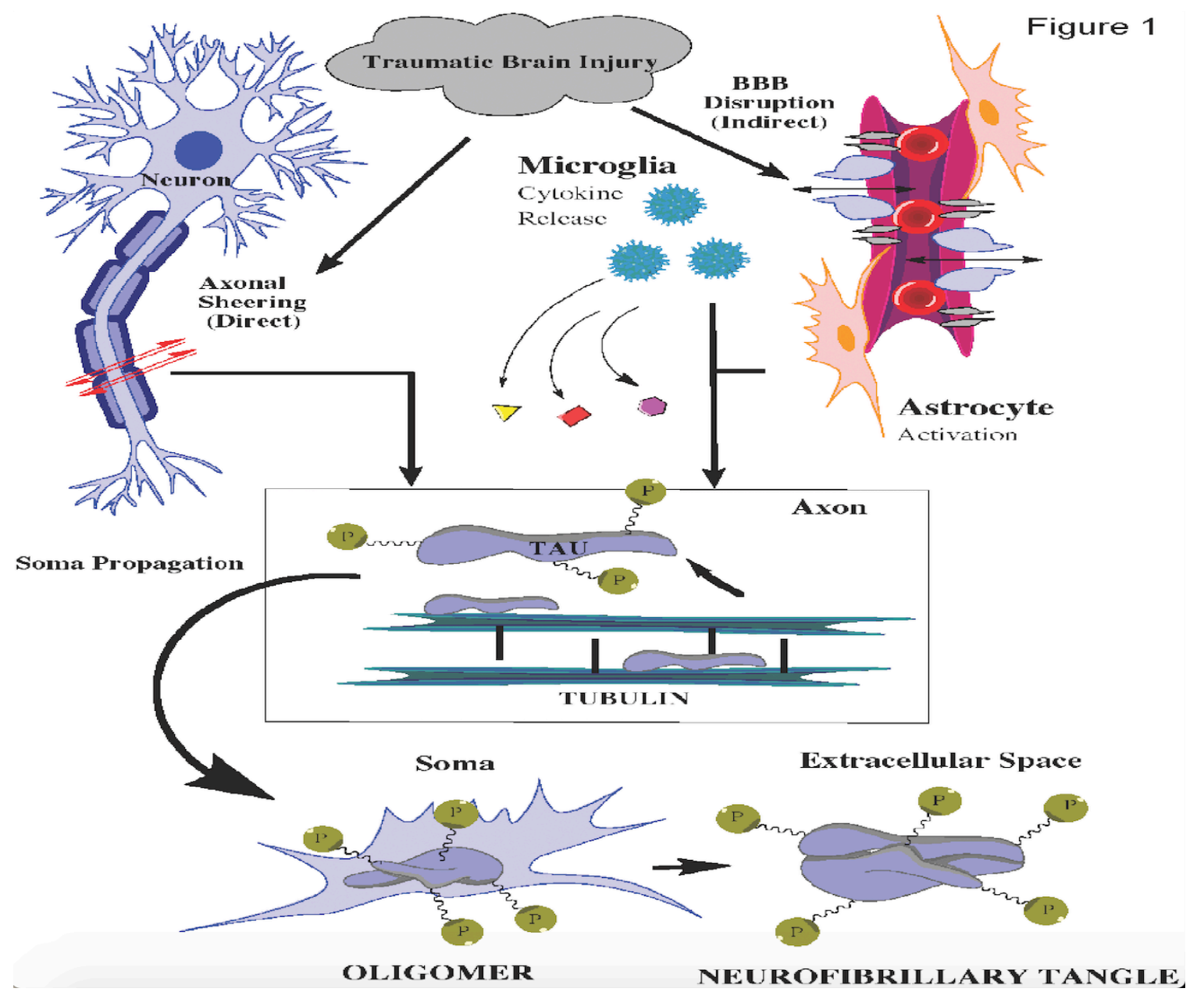

Figure 1.1: Traumatic Brain Injury can lead to diffuse traumatic axonal injury and blood brain barrier disruption. Shearing of axons results in the disruption of tau binding to tubulin. Subsequent hyperphosphorylation of tau leads to formation of tau oligomers in the neuronal soma. Eventually, neurofibrillary tangles form and are secreted into the extracellular milieu or spread to other neurons via trans-synaptic propagation. Concurrent with axonal shearing, traumatic brain injury can cause a rapid blood pressure spike resulting in blood brain barrier disruption. The disruption leads to an inflammatory cascade as well as microglia and astrocyte activation. Microglia and astrocyte activation in conjunction with tauopathy contribute to the pathology of chronic traumatic encephalopathy. 
A.

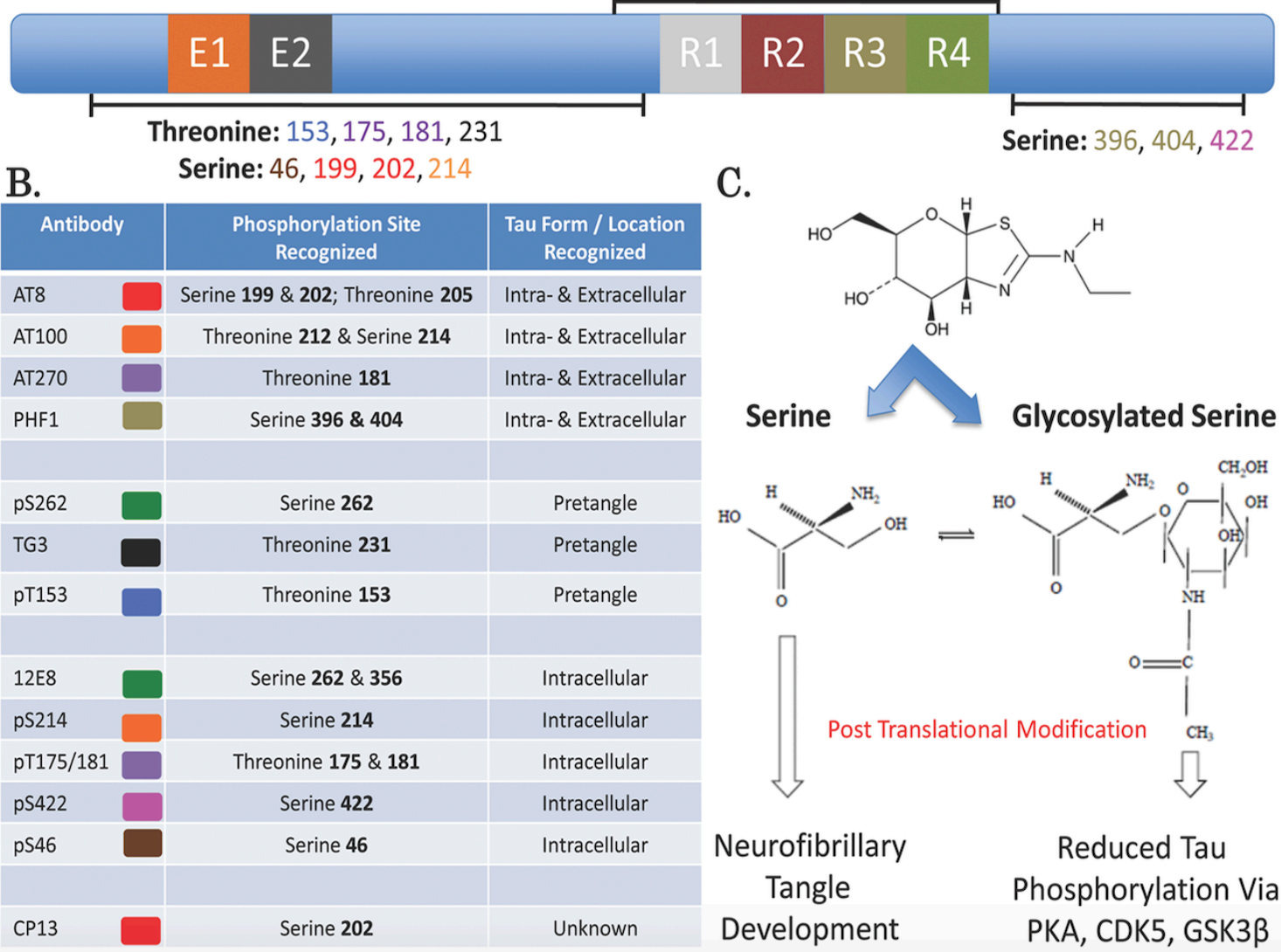

Figure 1.2: Tau is regulated by multiple biochemical processes including: nitration, glycosylation, ubiquitination, acetylation, sumoylation, and phosphorylation. A. We highlight some of the key regulation sites potentially involved in the pathophysiology of chronic traumatic encephalopathy. B. Each site has a specific antibody of interest that can be used to detect changes in intracellular/extracellular tau. C. Deglycosylation allows for conversion of tau tangles into bundles of straight filaments, thus increasing the accessibility of remaining tau located at microtubule edges. Glycosylation however reduces phosphorylation of PKA, CDK5, and GSK3B decreasing formation of neurofibrillary tangles. This example shows how post-translational modification of tau can regulate tau phosphorylation and ultimately lead to the development of neurofibrillary tangles, a hallmark of CTE. 


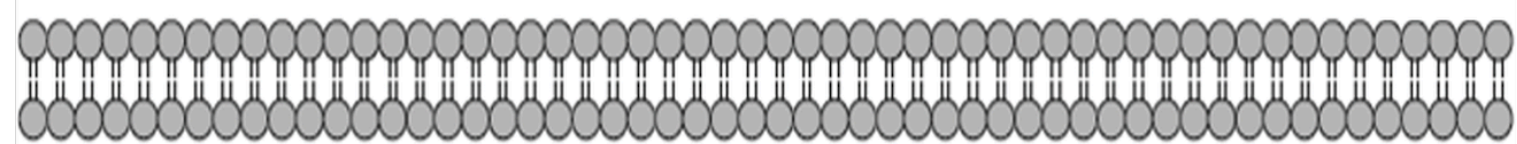

Intracelludar

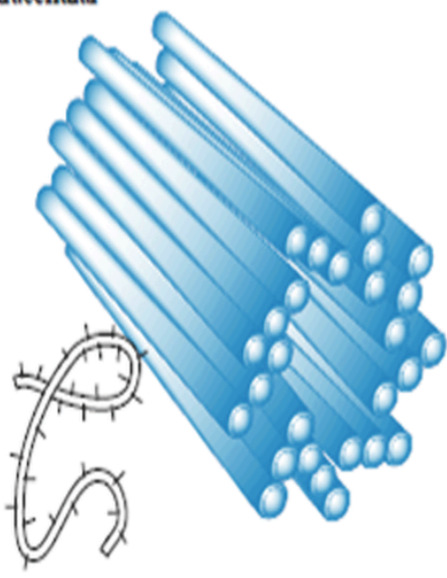

Microtubule-associated Tau Protein

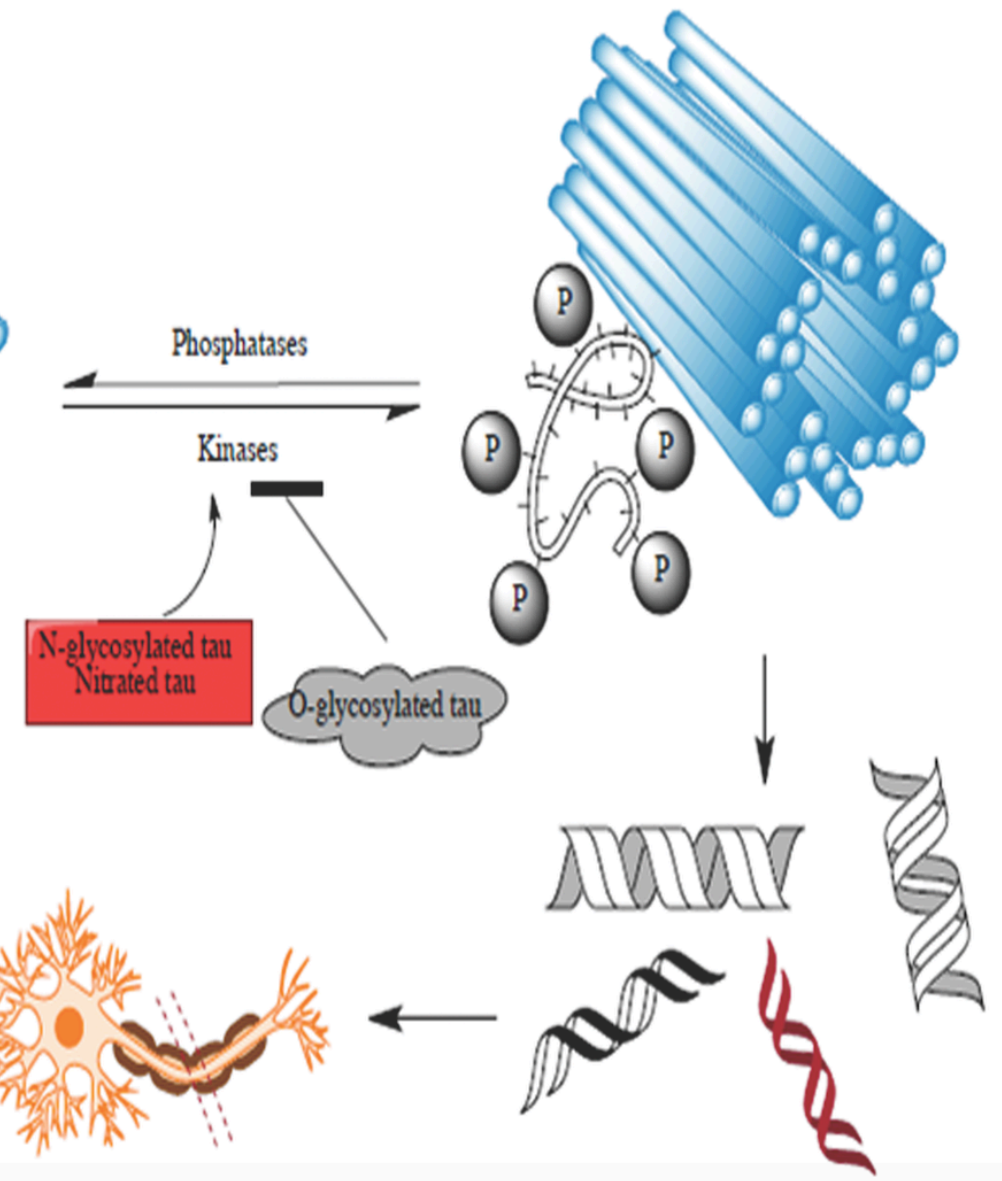

Neurodegeneration \& Impaired Axonal Transport

Neurofibrillary Tangles

Figure 1.3: Neurofibrillary tangle formation involves an imbalance between tau kinase and tau phosphatase activity. If tau kinase activity is increased and the phosphatase activity is decreased, hyperphosphorylation persists and can result in the formation of neurofibrillary tangles. Neurofibrillary tangles contribute to poor outcome by disrupting axonal transport and eventually causing the hierarchical spread of neurodegeneration. Neurodegeneration ultimately causes the classic symptoms seen in patients suspected of having chronic traumatic encephalopathy. 
Table 1.1

\begin{tabular}{|c|c|c|c|}
\hline Kinase Name: & Site of Regulation: & Physiological Role: & Activated by TBI: \\
\hline ERK1/2 & $\begin{array}{l}\text { Phosphorylation of } \\
\text { threonine-x-tyrosine } \\
\text { motif }\end{array}$ & $\begin{array}{l}\text { Important role in } \\
\text { growth factor } \\
\text { signaling, cell } \\
\text { survival, and } \\
\text { apoptosis }\end{array}$ & $\begin{array}{l}\text { Yes (Kuo et al } \\
2013)\end{array}$ \\
\hline CDK5 & $\begin{array}{l}\text { Binding to CDK } \\
\text { Receptor } 1 \text { or CDK } \\
\text { Receptor } 2\end{array}$ & $\begin{array}{l}\text { Plays a role in } \\
\text { neural development, } \\
\text { pain signaling, and } \\
\text { sensory processing }\end{array}$ & Yes (Li et al 2013a) \\
\hline GSK3- $\beta$ & $\begin{array}{l}\text { Requires priming } \\
\text { kinase to } \\
\text { phosphorylate a } \\
\text { substrate prior to } \\
\text { phosphorylation at } \\
\text { tyrosine-216. } \\
\text { Phosphorylation at } \\
\text { serine-9, however, } \\
\text { hides the active site }\end{array}$ & $\begin{array}{l}\text { Implicated in } \\
\text { neuronal } \\
\text { development, } \\
\text { glucose } \\
\text { homeostasis, and } \\
\text { body pattern } \\
\text { organization }\end{array}$ & $\begin{array}{l}\text { Yes (Shapira et al } \\
\text { 2007) }\end{array}$ \\
\hline PKC & $\begin{array}{l}3 \text { categories based on } \\
\text { binding at C-terminal: } \\
\text { conventional requires } \\
\text { diacyl-glycerol and }\end{array}$ & $\begin{array}{l}\text { PKC activity is } \\
\text { involved with } \\
\text { learning and } \\
\text { memory, regulation }\end{array}$ & $\begin{array}{l}\text { Yes (Zohar et al } \\
\text { 2011) }\end{array}$ \\
\hline
\end{tabular}




\begin{tabular}{|c|c|c|c|}
\hline & $\begin{array}{l}\text { calcium for } \\
\text { activation, novel } \\
\text { requires diacyl- } \\
\text { glycerol, and atypical } \\
\text { does not require } \\
\text { calcium or } \\
\text { diacylglycerol. Once } \\
\text { active, the receptors } \\
\text { for activated C-kinase } \\
\text { bind PKC and help } \\
\text { translocate it to the } \\
\text { plasma membrane }\end{array}$ & $\begin{array}{l}\text { of transcription, } \\
\text { controlling cell } \\
\text { growth, and } \\
\text { mediating immune } \\
\text { responses }\end{array}$ & \\
\hline JNK & $\begin{array}{l}\text { Diphosphorylation of } \\
\text { the threonine-proline- } \\
\text { tyrosine motif }\end{array}$ & $\begin{array}{l}\text { JNKs participate in } \\
\text { multiple stress } \\
\text { cascades, the } \\
\text { inflammation } \\
\text { response, and } \\
\text { reactive oxygen } \\
\text { species formation }\end{array}$ & $\begin{array}{l}\text { Yes (Tran et al } \\
\text { 2012) }\end{array}$ \\
\hline AKT & $\begin{array}{l}\text { Akt binds to } \\
\text { phosphatidylinositol } \\
(3,4,5) \text {-triphosphate } \\
\text { on the cell membrane }\end{array}$ & $\begin{array}{l}\text { Akt plays a role in } \\
\text { apoptosis, cellular } \\
\text { metabolism, and cell } \\
\text { migration }\end{array}$ & $\begin{array}{l}\text { Yes (Ksiezak- } \\
\text { Reding et al 2003) }\end{array}$ \\
\hline
\end{tabular}




\begin{tabular}{|l|l|l|l|}
\hline and then is & & \\
phosphorylated at & & & \\
threonine 308 by & phosphoinosotide & & \\
kinase 1 & & \\
\hline
\end{tabular}

Table 1.1 Legend: Table 1.1 provides a list of tau kinases and the physiologic roles in which they function. In addition, it highlights if the overall levels of these kinases are altered by traumatic brain injury. 


\section{Chapter Two}

Elucidating the role of compression waves and impact duration for generating mild traumatic brain injury in rats

Submitted to Brain Injury: accepted pending minor revisions.

Lucke-Wold BP, Phillips M, Turner RC, Logsdon AF, Smith KE, Huber JD, Rosen CL, Regele JD 


\section{ABSTRACT}

1.7 million concussions occur each year in the United States. The mechanisms linking acute injury to chronic deficits are poorly understood. Mild traumatic brain injury has been described clinically in terms of acute functional deficits, but the underlying histopathologic changes that occur are relatively unknown due to limited high-function imaging modalities. In order to improve our understanding of acute injury mechanisms, appropriately designed preclinical models must be utilized. The clinical relevance of compression wave injury models revolves around the ability to produce consistent histopathologic deficits. Repetitive mild traumatic brain injuries activate similar neuroinflammatory cascades, cell death markers, and increases in amyloid precursor protein in both humans and rodents. Humans however infrequently succumb to mild traumatic brain injuries and therefore the intensity and magnitude of impacts must be inferred. As accelerometers become more widely used in athletics, it is imperative that the physical and mechanical properties of pre-clinical concussion models be determined. This increased understanding could link the histopathologic deficits seen in rodents to what might be happening in human brains following repetitive concussions. Advances in mathematical and computer modeling can help characterize the wave properties generated by the compression wave model. While this concept of linking duration and intensity of impact to subsequent histopathologic deficits makes sense, numerical modeling of compression waves has not been performed in this context. In this collaborative interdisciplinary work, numerical simulations were performed to study the creation of compression waves in our experimental model. This work was conducted in 
conjunction with a repetitive compression wave injury paradigm in rats in order to better understand how the wave generation correlates with validated histopathologic deficits.

KEY WORDS: Finite Volume Modeling, Compression Wave, Traumatic Brain Injury, Apoptosis, Pre-clinical Model

\section{INTRODUCTION}

It has been estimated that 1.7 to 3.4 million concussions occur each year in the United States (McClure et al 2014). How mechanical forces cause brain injury and initiate injury cascades is poorly elucidated. Therefore, a diagnosis of concussion is primarily based on observable symptomatic changes (McCrea et al 2003). It is currently unknown how many athletes and soldiers are at risk for developing chronic traumatic encephalopathy (Bailes et al 2015). Chronic traumatic encephalopathy is a degenerative tauopathy characterized by mood disorders and cognitive deficits (McKee et al 2009). The biochemical mechanisms linking acute injury to behavioral deficits and the development of neurodegeneration over time warrants further investigation (Lucke-Wold et al 2014c). Banks and colleagues are conducting the first prospective cohort study to determine the incidence of chronic traumatic encephalopathy in mixed martial arts fighters (Banks et al 2014). Laboratory research can also provide important discoveries for how acute injury contributes to tauopathy. Most importantly, laboratory studies allow for a comparison of force dynamics to observed physiologic changes post-injury(Hua et al 2015).

In order to improve our understanding of mild traumatic brain injury, it is necessary to utilize pre-clinical models that produce relevant pathologic changes 
consistent with human brain injury. In previous papers, we have described acute biochemical changes that occur within a rat's brain following compression-wave injury. These changes include blood brain barrier disruption, endoplasmic reticulum stress, and oxidative stress (Logsdon et al 2014, Lucke-Wold et al 2014a, Lucke-Wold et al 2015a). Recently, we discovered that these acute pathways are implicated in the development of tauopathy, and contribute to long-term cognitive and behavioral deficits (Turner et al 2015). These deficits in humans can manifest as post-traumatic epilepsy, sleep disturbances, and increased risk for suicide (Lucke-Wold et al 2015b, Lucke-Wold et al 2015c, Wasserman et al 2008). In this multidisciplinary project, we sought to delineate the wave dynamics associated with our clinically relevant model and correlate the wave properties to observed histopathologic changes within the rat's brain post-injury. Simulation and numerical modeling can be used to enhance our understanding of the wave properties and impact force experienced by the rat. These properties are critical for determining how mechanical injury causes lasting damage that ultimately leads to behavior changes.

\section{METHODS}

\section{Tabletop model}

The model consists of steel driven and driver section with a mylar membrane sandwiched in-between. The driven and driver section are screwed tight prior to rupturing the membrane. The apparatus is securely attached to a table and the 0.005 " membrane was scored with a hand press prior to use. The intensity of the compression wave was determined by a pressure gage attached to a regulator. Nitrogen gas was used to rupture 
the membrane. The pressure was allowed to build via a needle nose attachment and regulator prior to rupture. The compression tube has a short driver section in order to generate a short-duration wave. Piezoelectric sensors (Model 102AO5; PCB Piezotronics) were placed in the reflected and two incident positions near the exit of the shock tube to produce a linear configuration as shown in figure 2.1. To test for planarity, the three sensors were placed at the edge of the tube. Data was recorded using a sensor signal conditioner (482C Series; PCB Piezotronics) and a data acquisition board (DAQ 23GF; National Instruments). A computer program written with LabView version 12.0 (National Instruments) was used for analysis. The anesthetized rats head was placed at the same position as sensor two. A polyvinyl tube to protect the thoracic cavity from wave exposure protected the body.

\section{Numerical approach}

Numerical simulations are used to reconcile pressure profiles with the experimental setup in order to characterize wave formation. The tube and the surrounding air may generally be modeled by the compressible Navier-Stokes equations describing fluid flow. The flow produces a shock wave and an expansion wave propagating into stationary flow at atmospheric conditions. The timescales of both phenomena are small relative to the timescale of the viscous/diffusive terms within the Navier-Stokes equations. It follows that the boundary layers formed near the interior of the compression tube will have a minimal influence on the flow. Thus, the non-viscous Euler equations are used to simulate the compression waves generated from the tabletop compression tube. 
The Euler equations listed in conservation form are:

$$
\partial \boldsymbol{U} \partial t+\partial \boldsymbol{F} \boldsymbol{x} \boldsymbol{U} \partial x+\partial \boldsymbol{F} \boldsymbol{y} \boldsymbol{U} \partial y=0 .
$$

where,

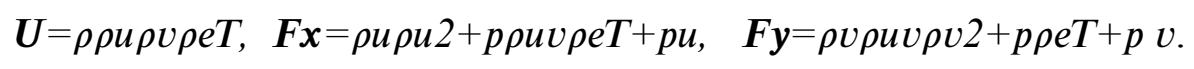

The conservative variables inside the vector $\boldsymbol{U}$ represent density, $\rho$, x-momentum, $\rho u$, ymomentum, $\rho v$, and total energy, $\rho e T$. The total energy per unit mass may be defined as the summation of the internal and kinetic energy:

$$
e T=p \rho \gamma-1+12 u 2+v 2 \text {. }
$$

An ideal gas equation of state $P=\rho$ Rair $T$ is used to close the system of equations.

The compression tube is modeled using an axisymmetric geometry, which reduces computation time by solving for a three-dimensional flow field on a twodimensional domain. The computational domain illustrated in figure 2.2 shows the compression tube with a large domain at the compression tube exit to capture the propagation of the blast wave after it exits the tube. The surrounding fluid region is chosen to be large enough to capture the transient evolution of the compression wave past the sensor two location without experiencing any interactions with the domain boundaries until much later.

The burst of the diaphragm is modeled by using a discontinuous initial condition where the pressure is higher in the driver section and lower outside. The initial velocity is zero and the initial temperature is $20^{\circ} \mathrm{C}$ everywhere. The initial conditions across the 
diaphragm are $110 \mathrm{psi}$ in the driver section at atmospheric conditions, with $14.7 \mathrm{psi}$ in the driven section and the domain outside the tube.

The governing equations are solved numerically using Star-CCM+. The WeissSmith Precondition Roe's Scheme (Weiss et al 1999) is used to discretize the physical domain into cells, and the governing equations are solved with a finite volume scheme. The scheme uses a multidimensional linear reconstruction to calculate the fluxes at each cell edge. The reconstruction uses a Taylor series expansion at the cell face to retain higher order accuracy. A multiple stage Runge-Kutta second order explicit scheme is utilized for the time integration (Huang \& Ayoub 2008). The time-step used in each simulation is determined using the Courant-Freidrich-Lewis (CFL) number. The CFL number is defined

$$
C F L=(u+c) \Delta t \Delta x,
$$

where $u$ and $c$ are local fluid velocity. The maximum CFL inside the domain was used to choose the time-step. The CFL number used for the simulations in this work is 0.5 .

A range of grid spacing is used in this work and range from $0.3175 \mathrm{~cm}$ to 0.0595 $\mathrm{cm}$. A grid sensitivity study is performed to ensure that the solution is not grid dependent. Computing the $L 2$ norm assesses grid sensitivity. The $L 2$ error is typically calculated by comparing the solution for the different mesh refinements to an exact solution. Since no exact solution is available, the solution with the finest mesh is used as an exact solution.

The $L 2$ error may be represented as, 


\section{$L 2=\int P M e s h-P E x a c t 2 d x 0.5$}

The $L 2$ error value represents the error over an entire domain with individual contributions from each cell.

\section{$\underline{\text { Animals }}$}

The Institutional Animal Care and Use Committee at West Virginia University approved all experiments involving animals. The experiments were conducted in accordance with the Guide for the Care and Use of Laboratory Animals. Sixteen (16) young-adult male Sprague dawley rats (Hilltop Lab Animals, Inc.) were used for immunohistochemistry (IHC). The rats were divided into two groups: repeat injury (6 compression waves every other day over 2 weeks) and anesthetized (4\% isoflurane with $2 \%$ maintenance) control. Repeat injury exposure was performed with the $0.005 \%$ membrane according to the methods previously described (Turner et al 2013). A power analysis was conducted (DSS Research) with the equation $n=1+\left[2 \mathrm{C}(\mathrm{s} / \mathrm{d})^{2}\right]$, where $\mathrm{C}$ is a value dependent upon the $\mathrm{a}$ and $\mathrm{b}$ values chosen. For this study $\mathrm{a}=0.05$ and $\mathrm{b}=0.2$ for $\mathrm{a}$

power of $1-b=0.8 . C_{(0.05,0.8)}=7.85$ (obtained from table). The number needed is based on effect size and standard deviation, which was estimated from previous work. Rats were housed with a 12h:12h light to dark cycle and food and water available ad libitum.

\section{Immunohistochemistry}

Animals were anesthetized as described above followed by decapitation. The brain was removed and immediately flash frozen in isopentane and stored at $-80^{\circ} \mathrm{C}$. Brains were sliced to a thickness of $20 \mu \mathrm{m}$ using a cryostat. Standard Hematoxylin and 
Eosin, Fluorojade B, Thioflavin, and DAB (Sigma Aldrich) staining protocols were utilized to stain for cellular morphology and Amyloid precursor protein (APP) (Cell Signaling).

Images were obtained from a blinded observer using a Zeiss Axio Imager 2 and an Olympus MVX10 for brightfield and fluorescent imaging. For immunohistological DAB stains, 100 total cells were counted in the stereologic selected region of interest within the prefrontal cortex and determined to be either expressing or non-expressing. The ratios between groups were compared using chi-squared analysis. For fluoroscent stains, a blinded observer used image $\mathrm{J}$ quantification. The measurements were adjusted for background giving corrected total cell fluorescence. The total cell fluorescence was compared between groups.

\section{$\underline{\text { Statistical analysis }}$}

Data were analyzed using GraphPad Prism version 4.0. An observer blinded to experimental group did imaging quantification. A student's t-test was used to compare across control and repeat injury groups for fluorescent imaging. A $\chi^{2}$ analysis was used for DAB staining. A p-value of $<0.05$ was considered statistically significant for all studies.

\section{RESULTS}

\section{Compression wave formation}

Figure 2.2 shows a sequence of pressure contours, which illustrate the formation and propagation of a traditional blast wave. At $\mathrm{t}=0$, the diaphragm separates the fluid 
inside the driver section from the fluid inside the driven section. The fluid inside the driver section is at higher pressure than the atmospheric pressure in the driven section. Once the diaphragm bursts, the $t=0.1 \mathrm{~ms}$ frame shows that a shock wave moves to the left and an expansion wave moves to the right. At $\mathrm{t}=0.3 \mathrm{~ms}$ the shock wave has exited the tube and expands in all directions. The exit of the shock wave creates an expansion wave that moves inside the shock tube. The induced flow from the shock wave as it propagates to the left further away from the compression tube creates a low-pressure region behind the shock wave. This region is where the pressure drops below atmospheric pressure.

A one-dimensional axis is selected down the center of the compression tube to evaluate the error. The pressure values along the axis are compared between different mesh refinements at a physical time of $1 \mathrm{~ms}$. Figure 2.3 shows the $L 2$ error as a function of grid spacing on a log-log scale. The solution with the finest resolution is used as an exact solution. Figure 2.3 also shows that the slope is 1.841 , which indicates that the solution is converging at a rate between first and second order accuracy. This convergence rate is consistent with the scheme's accuracy since the reconstruction step makes the solution second order accurate everywhere except for discontinuities such as shocks and contact discontinuities.

Figure 2.4 shows a contour plot of pressure on an $\mathrm{x}$-t diagram using the solution data located along the axis in the center of the compression tube. The green dashed lines in figure 2.4 represent the analytical solution for an ideal one-dimensional compression tube. Looking exclusively at the shock wave and the head of the expansion wave propagation, both phenomena are represented relatively accurately compared to a purely 
1-D shock tube case. It may also be seen that the expansion wave reflects off the driver wall at $\mathrm{t}=0.4 \mathrm{~ms}$ and begins propagating in the direction of the shock wave. The solid blue lines represent the end of the compression tube and the initial diaphragm location. When the shock exits the compression tube at $\mathrm{t}=0.149 \mathrm{~ms}$ an expansion wave is created as the wave expands radially outward in all directions. The contour shows that the pressure behind the transmitted compression wave decays with distance from the tube exit. The red dashed lines represent the locations of each of the pressure transducers in the experiment.

\section{Pressure sensors}

Pressure readings are obtained using pressure taps at specific locations within the compression tube and at the location where the rat will be placed. Figure 2.1 provides the geometry and location of each of the sensors from the experiment. The pressures versus time profiles were analyzed for each pressure sensor placed linearly. The waves were mapped and quantified with MATLAB as previously published (Turner et al 2013). Three transducers were then placed in the same plane at the edge of the tube. These three transducers showed variation in the pressure histories, which suggests that non-planar shock waves are produced. This non-planar wave behavior is likely caused by inconsistent burst patterns of the diaphragm. The diaphragms are scored in similar patterns with a press to improve the planarity of the wave. Since there is some planar variability in pressure histories from case to case, an average pressure history is calculated using 7 membrane ruptures. The averaged pressure histories are then readily compared with the simulation results. 


\section{Pressure profiles}

The pressure profiles for each sensor are shown in figure 2.5 for both the averaged experimental data and the numerical simulation. The pressure histories of the experimental data were shifted to match time of peak pressure occurrence. The time shift allows an average between pressure profiles and direct comparison between the averaged experiment and the simulation.

The third sensor shows the same general trend between the experiment and the simulation. The over-pressure does not reach the peak value observed in the simulation and the rise time is finite. The lower peak pressure and more gradual pressure increase indicate a wave that more closely resembles a strong compression wave. This is likely due to incomplete or partial diaphragm rupture. However, since the pressure ratio across the diaphragm is sufficiently large to create a shock (approximately 7.5), the wave created inside of the tube from the diaphragm burst will still be regarded as a shock wave. It can also be seen that the time scale of the pressure recovery matches between the simulation and the experiment. This pressure recovery occurs from the shock exiting the tube and the expansion wave travels inside.

The first sensor is near the exit of the tube and shows a similar behavior between the experiment and the simulation. The peak of the simulation is almost twice the magnitude of the experiment. Similar to the third sensor, the first sensor pressure recovery timescale is consistent between the experiment and the simulation.

The second pressure sensor is oriented for the experiment as a reflected sensor where the pressure transducer is pointed into the flow direction. The pressure tap is held 
within a large metal plate approximately 2 inches from the end of the shock tube. As can be expected, the pressure trace for sensor two shows that the experiment and numerical model are consistent on the arrival time of the blast wave at the transducer two location. Beyond this time the two pressure histories deviate because the numerical simulation does not model a reflecting plate. Instead, the numerical simulation measures what the pressure profile would be in the absence of an object that alters the wave profile. In the experiment, the pressure rises much higher and retains that high pressure for approximately $1.5 \mathrm{~ms}$. This higher pressure rise occurs because of the wave reflection that occurs at sensor 2 from the wall plate. It should be noted that the reflection of the wave alters the wave profile to make the wave duration much longer than an unobstructed wave. This suggests that very different pressure histories may be recorded for the same compression wave depending upon the pressure transducer placement and geometry.

\section{Immunohistochemistry}

Compression wave TBI can lead to long-term changes in the brain. Neurons are acutely injured and can undergo apoptosis or survive with aggregated proteins. We show nuclear fragmentation, pyknotic nuclei, blebbing, and chromatolysis in the contralateral prefrontal cortex of repeat TBI animals compared to control animals (Figure 2.6 A-B). A significant increase in amyloid precursor protein was also seen 72 hours post repeat TBI $(41 / 100)$ compared to controls $(2 / 100)$ in the contralateral prefrontal cortex $\chi^{2}=81$, $\mathrm{p}<0.001$ (Figure 2.6 C-D). In addition to apoptosis, damaged tissue can degenerate over time. Fluorojade B was used to mark early signs of neurodegeneration. We found a significant increase in the contralateral prefrontal cortex at 72 hours post repeat TBI compared to controls $\mathrm{t}=8.96, \mathrm{p}<0.001$ (Figure $2.7 \mathrm{~A}-\mathrm{B}$ ). We also found a significant 
increase in fluorojade $\mathrm{B}$ in the contralateral hippocampus 72 hours post repeat TBI compared to controls $\mathrm{t}=3.22, \mathrm{p}<0.01$ (Figure $2.7 \mathrm{C}-\mathrm{D}$ ). The compression wave caused significant degeneration in the contralateral hemisphere due to rapid acceleration/deceleration forces. Finally, a significant increase in thioflavin staining was seen in the prefrontal cortex following repetitive TBI 72 hours post-injury compared to controls $\mathrm{t}=3.636, \quad \mathrm{p}<0.01 \quad$ (Figure $2.7 \quad \mathrm{E}-\mathrm{F}$ ). Thioflavin marks increased tau hyperphosphorylation and protein aggregation.

\section{DISCUSSION}

Panzer and colleagues recently urged for better understanding of pre-clinical TBI models and how these models produce head injury (Panzer et al 2012). Gupta proposed that the most clinically relevant understanding of TBI is to determine how compression waves penetrate into tissue (Gupta \& Przekwas 2013). The numerical simulations contained in this work show that the tabletop model produces a pressure wave consistent with a compression wave. The expansion wave is created after the shock wave exits the shock tube and combines with the shock wave to produce the compression wave. The simulation results correlated well with the model recordings for sensor one and sensor three. The wave duration for the numerical simulation at the sensor two location was five times shorter than that observed from the averaged pressure transducer history. This suggests that obstacles in the flow can significantly alter incident wave profiles. Compression waves such as these can cause rapid acceleration/deceleration contributing to damage at density gradients within the brain (Boulet et al 2011). This type of injury from compression waves is most frequently seen in clinics due to athletic concussions and motor vehicle collisions (DeKosky et al 2013). In this multidisciplinary paper, we 
show that the tabletop model creates compression waves and that these waves cause significant acceleration/deceleration injury measured with immunohistochemistry.

The acceleration/deceleration can cause shearing of vessels and axons ( $\mathrm{Su} \&$ Bell 2016). We observed significant increases in amyloid precursor protein and thioflavin after repetitive TBI showing damage from axonal shearing and persistent neuronal injury. Likewise H\&E and fluorojade B staining revealed increased cell death and nuclear damage consistent with compression wave penetration into tissue. The damage was particularly pronounced at the white/grey junction within the cortex and within the hippocampus. It is therefore representative of an impact producing substantial coup and contra-coup effects due to the acceleration/deceleration of the skull over time (Ommaya et al 1971). Readnower and colleagues observed substantial disruption of the blood brain barrier on the ipsilateral hemisphere following blast exposure likely due to a longer duration compression wave (Readnower et al 2010). Due to the rapid acceleration/deceleration seen with our model, we observe the disruption in the BBB on the contralateral hemisphere where the brain collides acutely with the skull (Lucke-Wold et al 2014a). The coup/contre-coup phenomenon is much more relevant to what is seen in the clinical setting instead of models using a fixed skull (Sato et al 2014).

Going forward, the use of advanced animal imaging can help determine the extent and progression of injury over time following exposure to repetitive compression waves (Tompkins et al 2013). Functional magnetic resonance imaging would help detect some of the more subtle differences produced by the injury that is not as readily detected by traditional assays. These changes could then be mapped in conjunction with advanced simulation techniques. Finite element and finite volume modeling are needed to further 
map the waves progression through the skull and into the brain (Jean et al 2014). Combining the computational modeling with in vivo injury data will continue to require interdisciplinary collaboration (Ganpule et al 2013). The development of these multidisciplinary teams is necessary to address the complexities of acceleration/deceleration neurotrauma. By developing better understanding of pre-clinical models, important injury factors relevant to the brain such as duration until degeneration, number of impacts needed for cognitive decline, and the time scale of acute injury cascades initiated post-injury can be determined. We provide evidence that compression waves cause acceleration/deceleration injuries consistent with those seen clinically.

\section{CONCLUSION}

The use of numerical modeling provides a unique understanding of the wave dynamics generated by our TBI model. The model reliably produces compression waves. The wave is of sufficient duration and force to cause both acceleration/deceleration and direct penetration. The injury profile outlined by immunohistochemistry confirms that cell death, axonal shearing, and tau hyperphosphorylation occurs following repetitive injury. These injury patterns may provide important clues for what is occurring in the brains of athletes following repetitive injuries. The use of advanced imaging will improve the ability to correlate between pre-clinical models and human pathology.

\section{ACKNOWLEDGMENTS}

A Neurosurgery Research and Education Foundation Medical Student Summer Fellowship, an American Medical Association Foundation Seed Grant, a Sigma Xi 
Grants-in-Aid of Research, and an American Foundation of Pharmaceutical Education Pre-doctoral Fellowship supported Brandon Lucke-Wold. 


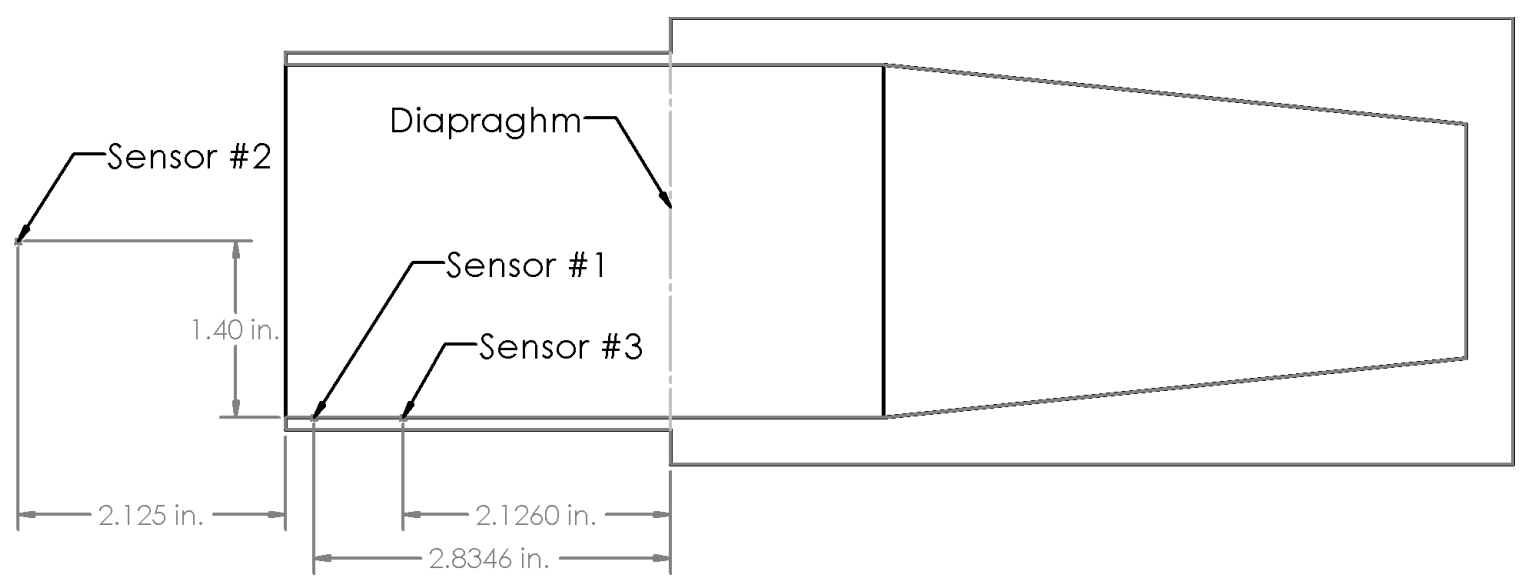

Figure 2.1: Geometry of Sensor Layout. A two dimensional representation shows the geometrical configuration of the problem. 

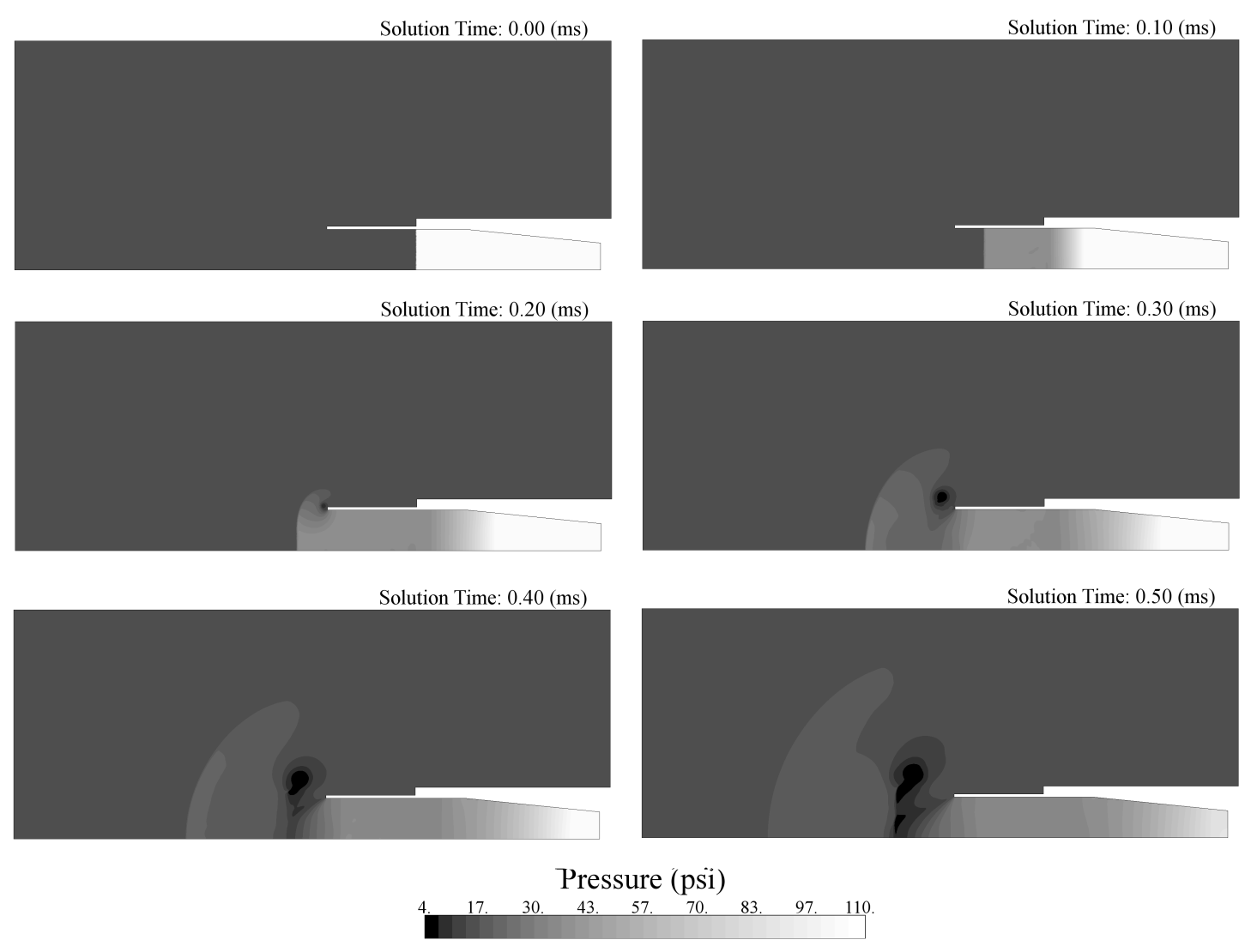

Figure 2.2: Contour Plot 2D Pressure. Domain pressure profile shown at different times provides initial conditions and follows the shock and expansion wave development and propagation. 


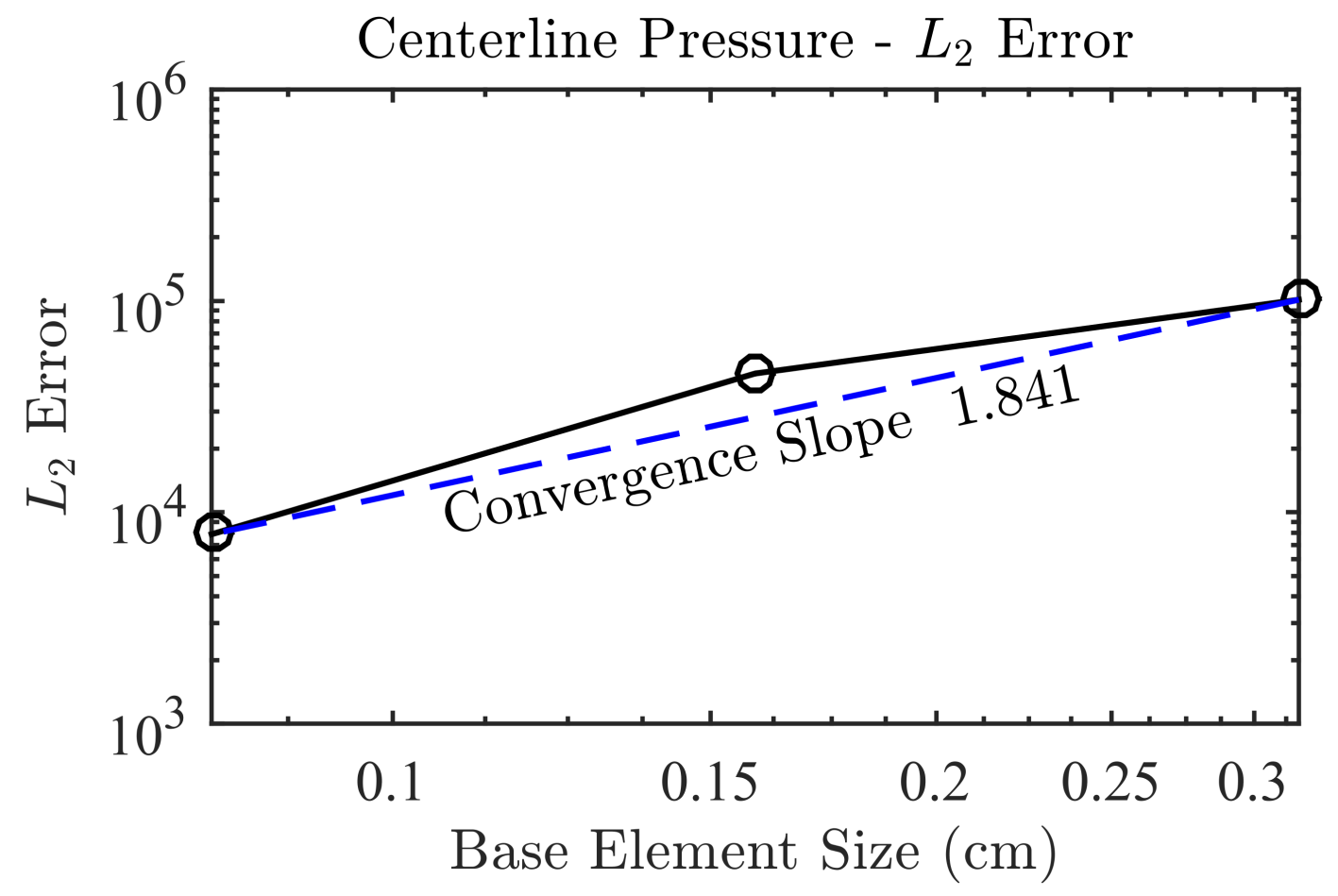

Figure 2.3: L2 Error. A log-log plot shows converging simulation results with increasing grid resolution. 


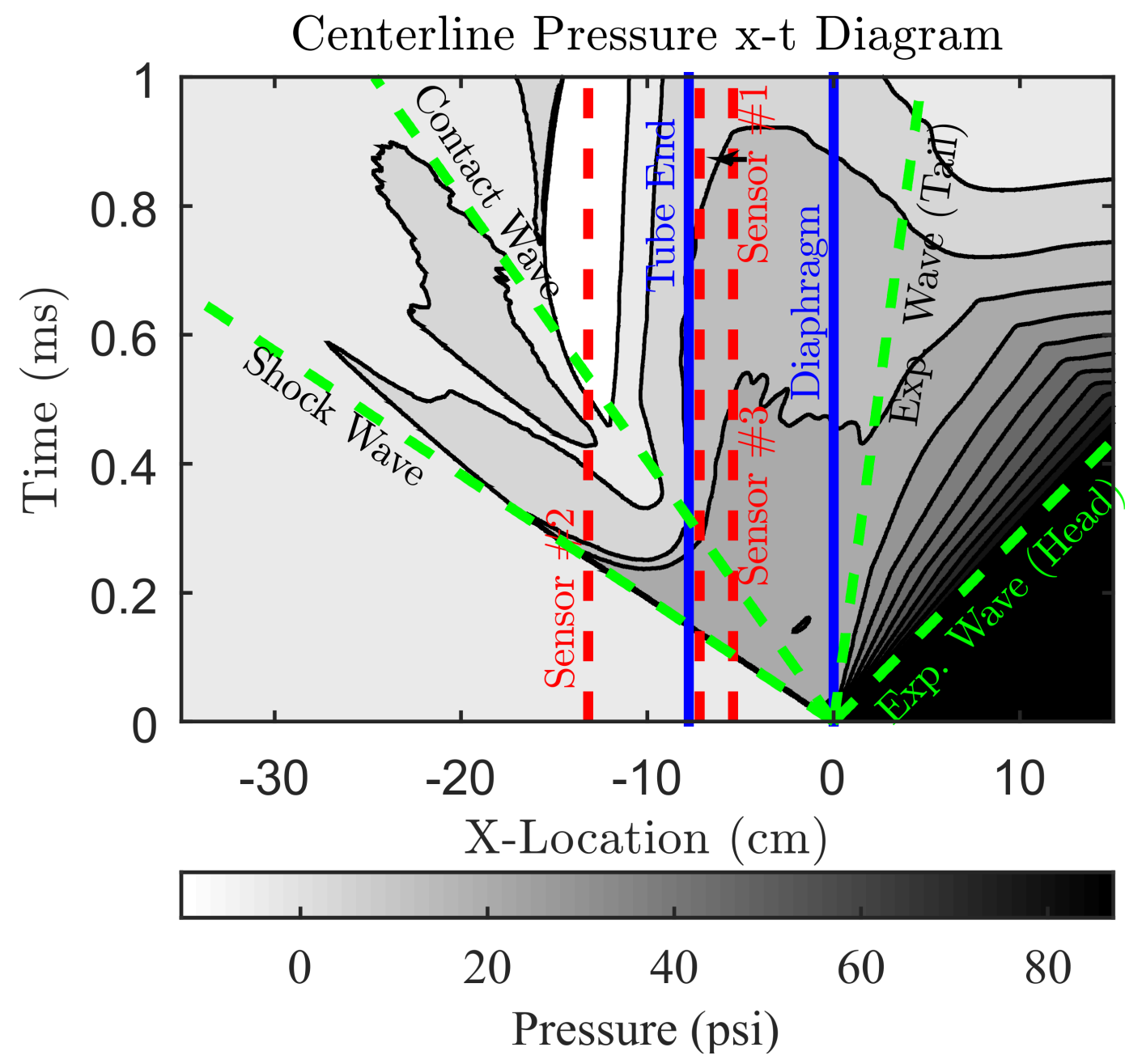

Figure 2.4: $x-t$ Diagram. An $x-t$ pressure contour plot shows the evolution of the shock and expansion waves after the diaphragm bursts. 

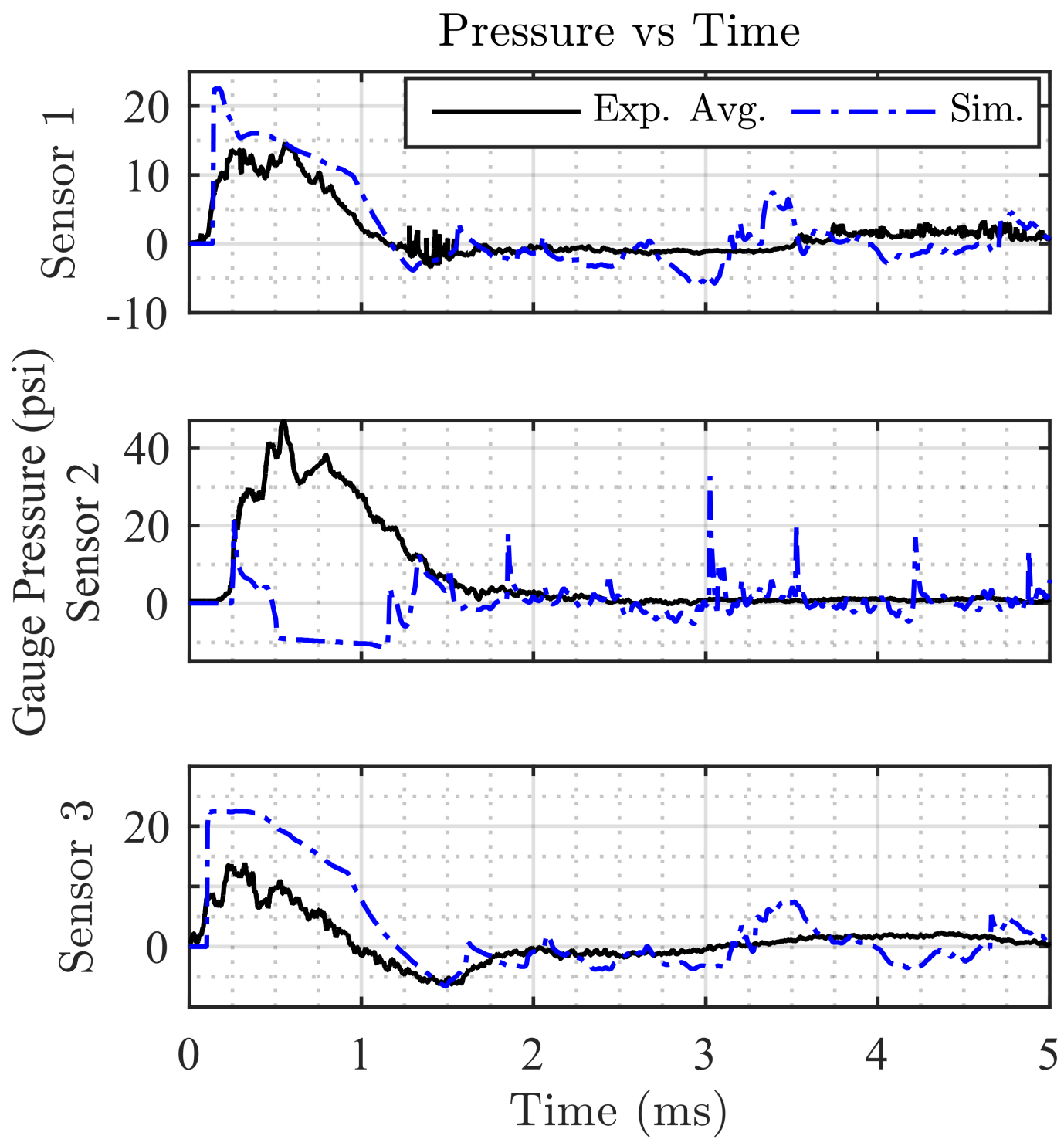

Figure 2.5: Pressure vs. Time by Sensor. A line plot provides a comparison between experiment and simulation. The experiment uses pressure taps to measure gage pressure while the simulation uses a point probe to measure static pressure. 

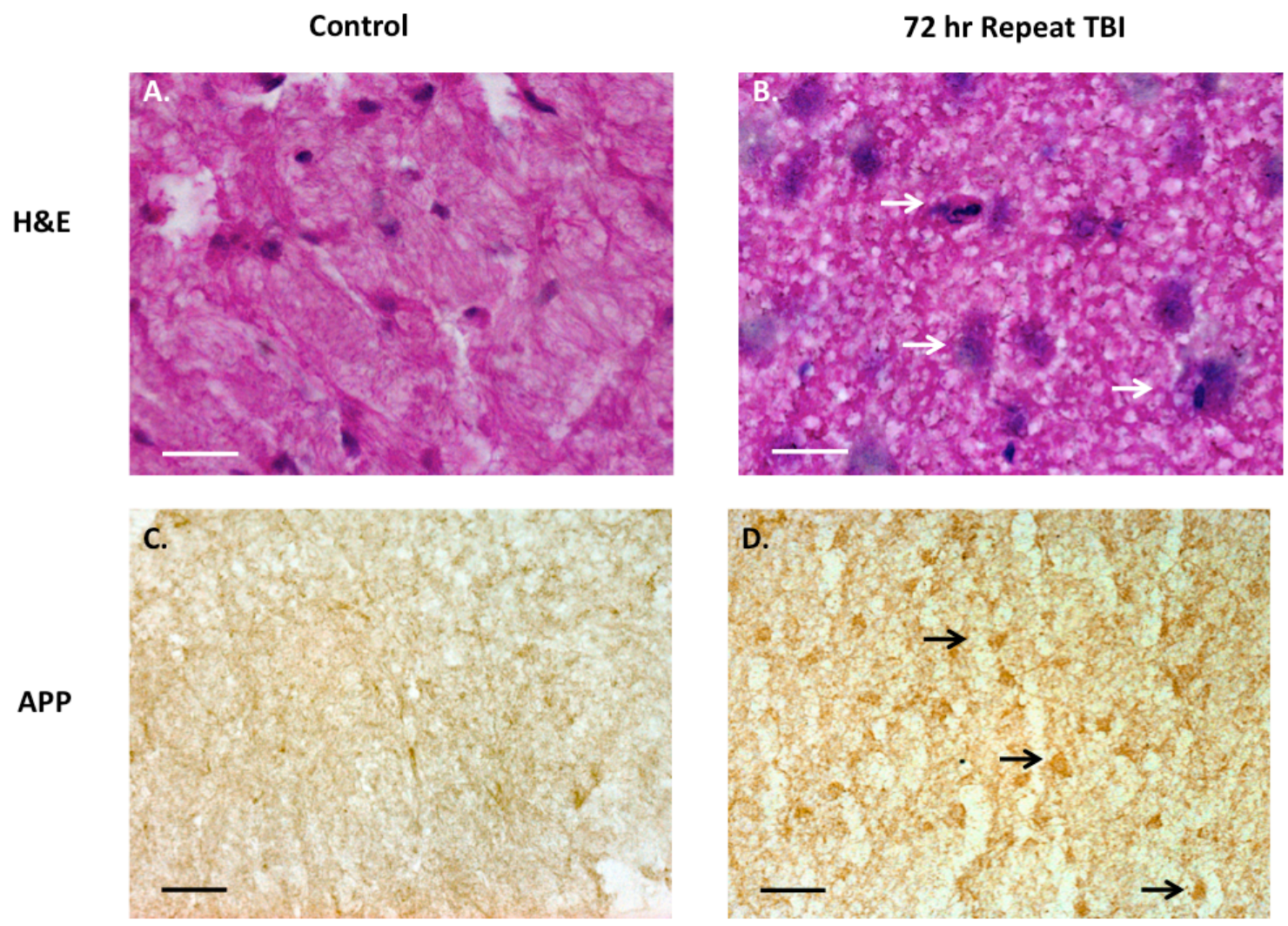

Figure 2.6: Acute Pathophysiology. H\&E staining reveals nuclear enlargement and fragmentation in the contralateral prefrontal cortex 72 hours post repetitive TBI. A significant increase in APP was also observed $\chi^{2}=81, \mathrm{p}<0.001$. Scale bar $=20 \mu \mathrm{m}$. 


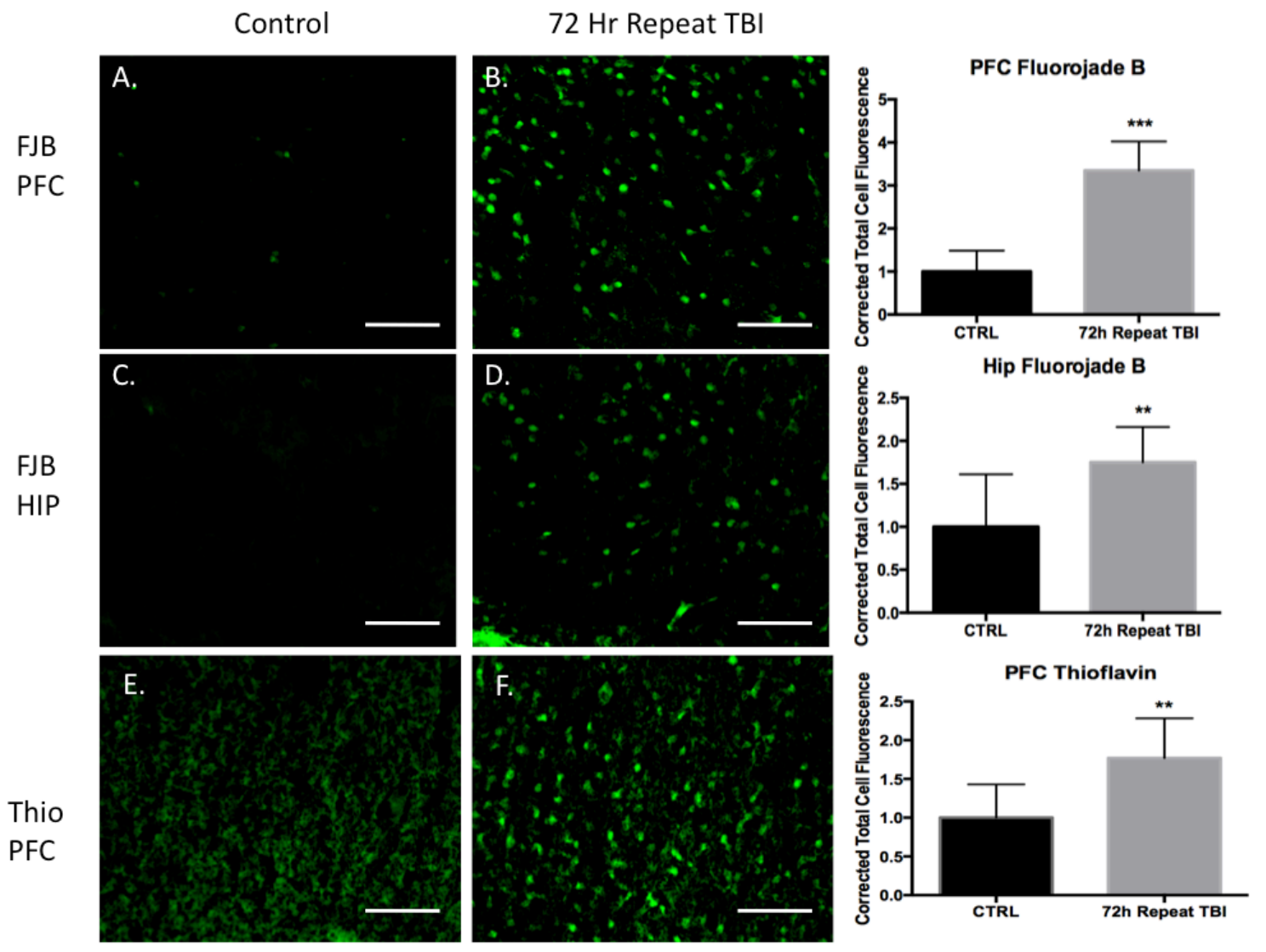

Figure 2.7: Neuronal Degeneration. A significant increase in fluorojade B was seen in the contralateral prefrontal cortex and hippocampus 72 hours following repetitive TBI. Thioflavin shows tau hyperphosphorylation and protein aggregation 72 hours following repetitive TBI. ${ }^{* *}=\mathrm{p}<0.01, * * * \mathrm{p}<0.001$. Scale bar $=50 \mu \mathrm{m}$. 


\section{Chapter Three}

The quest to model chronic traumatic encephalopathy: A multiple model \& injury paradigm experience

This work is published in Front Neurol. 2015 Oct 20;6:222. doi:

10.3389/fneur.2015.00222. eCollection 2015. PMID: 26539159

Turner RC, Lucke-Wold BP, Logsdon AF, Robson MJ, Dashnaw ML, Huang JH, Smith

KE, Huber JD, Rosen CL, Petraglia AL 


\section{ABSTRACT}

Chronic neurodegeneration following a history of neurotrauma is frequently associated with neuropsychiatric and cognitive symptoms. In order to enhance understanding about the underlying pathophysiology linking neurotrauma to neurodegeneration, a multi-model pre-clinical approach must be established to account for the different injury paradigms and pathophysiologic mechanisms. We investigated the development of tau pathology and behavioral changes using a multi-model and multiinstitutional approach, comparing the pre-clinical results to tauopathy patterns seen in post-mortem human samples from athletes diagnosed with chronic traumatic encephalopathy (CTE). We utilized a scaled and validated blast-induced traumatic brain injury model in rats and a modified pneumatic closed-head impact model in mice. Tau hyperphosphorylation was evaluated by western blot and immunohistochemistry. Elevated plus maze and Morris water maze were employed to measure impulsive-like behavior and cognitive deficits respectively. Animals exposed to single blast ( $\sim 50$ PSI reflected peak overpressure) exhibited elevated AT8 immunoreactivity in the contralateral hippocampus at 1 month compared to controls $(q=3.96, p<0.05)$. Animals exposed to repeat blast ( 6 blasts over 2 weeks) had increased AT8 $(q=8.12, p<0.001)$ and $\operatorname{AT270~}(q=4.03, p<0.05)$ in the contralateral hippocampus at 1 month post-injury compared to controls. In the modified controlled closed-head impact mouse model, no significant difference in AT8 was seen at 7 days, however a significant elevation was detected at 1 month following injury in the ipsilateral hippocampus compared to control $(q=4.34, p<0.05)$. Elevated plus maze data revealed that rats exposed to single blast (q $=3.53, \mathrm{p}<0.05)$ and repeat blast $(\mathrm{q}=4.21, \mathrm{p}<0.05)$ spent more time in seconds 
exploring the open arms compared to controls. Morris water maze testing revealed a significant difference between groups in acquisition times on days 22-27. During the probe trial, single blast $(\mathrm{t}=6.44, \mathrm{p}<0.05)$ and repeat blast $(\mathrm{t}=8.00, \mathrm{p}<0.05)$ rats spent less time in seconds exploring where the platform had been located compared to controls. This study provides a multi-model example of replicating tau and behavioral changes in animals and provides a foundation for future investigation of CTE disease pathophysiology and therapeutic development.

KEY WORDS: Chronic Traumatic Encephalopathy, Tauopathy, Animal Models, Cognitive Performance, Perivascular Pathology

\section{INTRODUCTION}

Current limitations in understanding CTE pathophysiology are unlikely to be addressed in a clinical population in the near future due to the challenges associated with establishing long-term, prospective cohort studies in such a population. Preclinical rodent models serve to fill this gap in knowledge for various disorders and could allow for further investigation of the molecular mechanisms responsible for CTE, as well as testing the potential of diagnostic and therapeutic approaches under development. Few preclinical models of CTE have been proposed that sufficiently demonstrate both the requisite tauopathy and behavioral changes attributed to CTE (Aungst et al 2014b, Bailes et al 2014, Blaylock \& Maroon 2011, Brody et al 2015, Creeley et al 2004, DeFord et al 2002, DeRoss et al 2002, Donovan et al 2014, Friess et al 2009, Fujita et al 2012, Genovese et al 2013, Goldstein et al 2012, Hawkins et al 2013, Klemenhagen et al 2013, Longhi et al 2005, Lucke-Wold et al 2014b, Mannix et al 2014, Mannix et al 2013, 
Mouzon et al 2012, Mouzon et al 2014, Petraglia et al 2014b, Petraglia et al 2014c, Shitaka et al 2011, Turner et al 2012a, Uryu et al 2002).

The purpose of this paper is to present two preclinical models that successfully reproduce some neuropathological and behavioral changes consistent with CTE-like phenotypes, and discuss future directions for CTE animal modeling. Brody and colleagues (Brody et al 2015) present several unanswered questions that we expand upon in our accompanying review such as the role of 1) inter-injury interval, 2) number of impacts, 3) impact severity, 4) age at time of impacts, 5) mechanism of impact, 6) genetics, 7) gender, and 8) effect of environment on the likelihood and/or progression of CTE development. We recently reported that endoplasmic reticulum stress might be a contributing factor linking acute neurotrauma to behavioral deficits (Logsdon et al 2014). The quest for elucidating CTE pathophysiology development is ongoing with the goal of targeting specific injurious cascades a key priority in order to prevent the emergence of clinical symptoms.

In this work we present novel models of neurotrauma-induced neurodegeneration in both mice and rats following exposure to single or repetitive brain injury, respectively. These models replicate both the tauopathy and some of the behavioral changes implicated in CTE in the clinical population. We believe the blast model is particularly relevant clinically due to utilization of a scaled, short-duration blast exposure. This is a striking contrast to long-duration blasts utilized in numerous studies that may more closely approximate an atomic blast than a blast from an improvised explosive device (Bass et al 2012, Panzer et al 2012, Panzer et al 2014a, Turner et al 2013). Park and colleagues show that by having the animal outside of the tube, the wave is directed towards the skull 
causing neurologic injury without extensive lung injury (Park et al 2011). Extended duration waves are generated when the tube has disproportionate length and volume ratios for the driven and driver sections. When the animals are placed within a tube that has not been scaled, the impulse exposure to the skull might not be representative of human exposure (Cho et al 2013). The modified closed-head injury in unanesthetized animals described previously by Petraglia and colleagues offers the benefit of injuring awake animals, more closely replicating the clinical picture seen in athletes than most other TBI models (Petraglia et al 2014b, Petraglia et al 2014c). Further studies are required to address injury paradigms that do or do not contribute to CTE development but utilization of these models appears promising in not only modeling CTE but also identifying therapeutic targets based upon other recently published work by our groups (Logsdon et al 2014, Lucke-Wold et al 2014a).

\section{METHODS}

\section{Animals and human samples}

All experiments involving animals were approved by either the Institutional Animal Care and Use Committee of West Virginia University or that of the University of Rochester and were performed based upon principles of the Guide for the Care and Use of Laboratory Animals. Fifty-six (56) young-adult male rats (300-350 grams) were acquired from Hilltop Laboratories (Scottdale, PA). All blast procedures were performed at West Virginia University. Twelve (12) young-adult male C57BL/6J mice were acquired from Jackson Laboratories (Bar Harbor, ME) and used for modified controlled cortical impact at the University of Rochester. All animals were allowed to acclimate 
upon arrival for 1 week prior to any experimentation. At all times animals were provided food and water ad libitum and maintained on a 12 hour light-dark cycle. Human samples were from deceased professional athletes that were previously diagnosed with chronic traumatic encephalopathy (Omalu et al 2010a). The tissue was collected from the entorhinal cortices.

\section{Experimental groups}

Fifty-six $(\mathrm{n}=56)$ rats were divided into three primary experimental groups for behavior - anesthetized controls $(\mathrm{n}=24)$, a single blast injury $(\mathrm{n}=16)$, and repeat blast injuries $(n=16)$. Each of these groups was sacrifed at 1 month following the final blast or sham-injury and after undergoing functional assessment. Elevated-plus maze was done at 7 days post-blast ( $\mathrm{n}=24 ; 8$ controls, 8 single blast, and 8 repeat blast) and the Morris water maze started at 21 days post-blast $(\mathrm{n}=32 ; 16$ controls, 8 single blast, and 8 repeat blast). Following behavioral analysis, rats from the EPM group were divided into two separate groups with one utilized for immunohistochemistry $(\mathrm{n}=10 ; 4$ controls, 3 single blast, and 3 repeat blast $)$ and the other for western blotting $(n=14 ; 4$ controls, 5 single blast, and 5 repeat blast). Twelve (12) mice were divided into three primary experimental groups - anesthetized controls $(n=4)$, single injury with sacrifice at one week $(n=4)$, and single injury with sacrifice at one month $(n=4)$. All mice were utilized for western blotting techniques at time of sacrifice. An experimental schematic can be seen in figure

\section{1.}

\section{$\underline{\text { Traumatic brain injury }}$}


Briefly, all blast injuries administered to rats as part of this work were of moderate intensity ( $\sim 50$ PSI peak reflected overpressure) as determined previously and completed under isoflurane-based anesthesia (Logsdon et al 2014, Lucke-Wold et al 2014a, Turner et al 2013). Blast exposure occurred on the right side of the animal and was only administered to the head and neck region. The rat was outside of the blast tube and a rigid barrier protected the remainder of the animal. Blast waves were of a shortduration ( $\sim 2 \mathrm{~ms}$ ) to ensure clinical relevance based on elucidated scaling parameters (Figure $3.2 \mathrm{~A}$ ). The scaling parameters are based on impulse dynamic measurements, which are more representative of human blast than closed models based on duration (Needham et al 2015).

Mice were utilized in a manner previously described (Petraglia et al 2014b, Petraglia et al 2014c). Briefly, mice were placed, unanesthetized, into a rodent restraint bag/cone and immobilized on top of a foam bed of known spring constant. A helmet was secured to the head using an elastic band, allowing for administration of a diffuse impact through force distribution. The helmet is made of stainless steel and measures $3 \mathrm{~mm}$ in thickness and $6 \mathrm{~mm}$ in diameter. Impacts were delivered using a modified controlled cortical impact device that was adjusted to include an altered tip of vulcanized rubber (Figure $3.2 \mathrm{~B}$ ). The impact was zeroed so that it was directly perpendicular to the helmet surface and orthogonal to the skull. The tip was driven $1 \mathrm{~cm}$ past zero point with a $100 \mathrm{~ms}$ impact.

\section{Western blot}


Animals were anesthetized and sacrificed by rapid decapitation. Brains were extracted and immediately placed in a lysate buffer with protease and phosphatase inhibitors as described elsewhere (Logsdon et al 2014). Brains were dissected and tissue flash frozen and stored at $-80{ }^{\circ} \mathrm{C}$ prior to blotting. Hippocampal protein samples were dissolved in $0.5 \mathrm{~mL}$ of $1 \%$ sodium dodecyl sulfate (SDS) prior to sonication and protein assay. Pre-cast 10\% 12-well gels (Life Technologies, Carlsbad, CA) were loaded with 30 $\mu \mathrm{g}$ of protein per well and run with $2 \mathrm{X}$ Lammeli buffer. Wet transfer was performed using nitrocellulose membranes (Bio-Rad, Contra Costa, CA) at 60 volts for 2.5 hours. Primary antibodies against AT8 (Pierce; Rockford, IL), AT270 (Pierce; Rockford, IL), CP13 (kindly supplied by Dr. Peter Davies), and PHF-1 (kindly supplied by Dr. Peter Davies) were utilized and detected utilizing the corresponding secondary. Membranes were imaged using a LI-COR fluorescent scanner (LI-COR; Lincoln, NE) and images converted to gray scale. Analysis was performed using background subtraction (Odyssey Processing Software, LI-COR) and values normalized to $\beta$-actin levels, resulting in a normalized intensity value.

\section{$\underline{\text { Immunohistochemistry }}$}

For immunohistochemistry preparation, animals were anesthetized using isoflurane and transcardially perfused with ice-cold physiologic saline followed by $10 \%$ formalin for a total of 10 minutes. Brains were extracted and placed in fresh $10 \%$ formalin for a minimum of 24 hours prior to blocking and subsequent paraffin embedding. Sections were prepared in $6 \mu \mathrm{m}$ thickness using a Leica RM2235 microtome (Leica Microsystems, Wetzlar, Germany). Staining was performed using standard protocols used within the field and previously by our laboratory, using the antibodies 
described above as well as thioflavin for detecting neurofibrillary tangles (Goldstein et al 2012, McKee et al 2010, Stern et al 2013).

\section{$\underline{\text { Behavioral assessments }}$}

Learning and memory was assessed using the Morris water maze. Spatial acquisition trials began at Day 21 after the final blast exposure (in both single and repeat injury paradigms). The pool utilized was $\sim 180 \mathrm{~cm}$ in diameter and filled with water at ambient temperatures $\left(18-21^{\circ} \mathrm{C}\right)$. A platform $(10 \mathrm{~cm} \times 10 \mathrm{~cm})$ was submerged $2.5 \mathrm{~cm}$ below the surface of the water. A series of objects was placed in the environment around the pool to provide visual cues for the animal during trials. The training paradigm (spatial acquisition) consisted of 6 days with a total of 4 trials occurring each day per animal. Animals were placed into the maze from four different locations each day (4 trials) with a total of 2 minutes (maximum) allowed per trial. Upon finding the platform, animals were allowed 15 seconds for acquisition to occur. If unsuccessful in finding the platform, animals were placed on the platform at the conclusion of 2 minutes by the investigator. On the probe trial day (platform removed), animals were placed in the maze at a novel location and allowed to explore the maze for 1 minute. Data was acquired using AnyMaze ${ }^{\mathrm{TM}}$ video tracking software (Stoelting Co., Wood Dale, IL) throughout all studies and allows for acquisition of latency, distance, and speed data to be analyzed across maze regions/quadrants.

Impulsivity was determined, at 7 days after the final blast, as previously described using an elevated-plus maze and measuring exploratory behavior (Johnson et al 2013, Logsdon et al 2014, Mosienko et al 2012). The apparatus was placed at a height of $60 \mathrm{~cm}$ 
from the floor and consisted of two open and two closed arms, with open arms opposing one another and intersecting perpendicularly with the opposed closed arms. Each arm was $50 \mathrm{~cm}$ long by $10 \mathrm{~cm}$ wide. Open arms were surrounded by clear plastic edging $\sim 1.5$ $\mathrm{cm}$ high. Closed arms were encased with black walls $30 \mathrm{~cm}$ tall, creating a 3-sided and comforting enclosure for the rodent. At the start of each 5-minute trial, animals were placed in the middle of the intersecting arms facing an open arm prior to release. Animals were allowed to explore the apparatus for the duration of the trial. AnyMaze ${ }^{\mathrm{TM}}$ software was utilized to record the animals' position, distance traveled, and entry pattern into various arms throughout the trial. An increased percentage of time spent in the open arms was considered a sign of impulsive behavior (Johnson et al 2013, Logsdon et al 2014, Mosienko et al 2012).

\section{Statistical analysis}

An observer blinded to experimental condition performed all data acquisition. One-way Analysis of Variance (ANOVA) was used for statistical analysis of all tests except spatial acquisition trials of the Morris water maze and immunohistochemical comparisons in which a two-way, repeated measures ANOVA and students t-test were utilized, respectively. Bonferroni post-hoc comparison was used to determine differences between experimental groups on two-way ANOVA with repeated measures for spatial acquisition. For all other comparison's, a Tukey's post-hoc was utilized. Analysis was completed using GraphPad Prism 5.0 (GraphPad Software, Inc.; La Jolla, CA). A p $<0.05$ was considered statistically significant for all data analyzed.

\section{RESULTS}


Neurotrauma induces tau hyperphosphorylation in both blast and closed-head injury

$\underline{\text { models }}$

To elucidate the effect of neurotrauma on the development of CTE-like neuropathology, animals were exposed to sham-injury, single-injury (either blast or modified closed-head), or repeat-injury (blast). Animals were first assessed for tau phosphorylation, using AT8 and AT270 antibodies, believed to be the initial precursor of neurofibrillary tangle development. AT8 forms a single band in rats and a double band in mice (Hawkins et al 2013). Conversely, AT270 forms a double band in rats, but only a single band in mice (Green et al 2007). The number of bands is indicative of calpain dependent phosphorylation, which is regulated uniquely between rats and mice (Medeiros et al 2012). Increased phosphorylation of tau was observed after blast exposure and modified closed-head injury but the location of the increase was model-dependent. Specifically, tau phosphorylation was observed in the contralateral hemisphere in rats exposed to repeat blast injury but only in the ipsilateral hemisphere, and only with the AT8 antibody, in mice receiving a single modified closed-head injury.

In rats exposed to blast, no significant difference in tau phosphorylation was observed in the ipsilateral hippocampus after blast injury for AT8 (Figure $3.3 \mathrm{~A}$ ) or AT270 (Figure 3.3 E). A significant difference was observed in AT8 $\left(\mathrm{F}_{2,11}=16.64, p<\right.$ $0.001)$ and $\operatorname{AT} 270\left(F_{2,9}=4.37, p<0.05\right)$ levels in the contralateral hippocampus of rats exposed to blast injury. At one month following a single blast, a significant increase in tau phosphorylation recognized by AT8 increase was measured compared to control $(\mathrm{q}=$ 3.96, $p<0.05$ ) (Figure 3.3 B). A significant increase in phosphorylation detected by AT8 was also observed after repeat blast $(\mathrm{q}=8.12, p<0.001)$ (Figure $3.3 \mathrm{~B})$. 
In the modified closed-head injury model utilized on the mice, a significant difference was observed in tau phosphorylation AT8 $\left(\mathrm{F}_{2,9}=4.93, p<0.05\right)$ in the ipsilateral hippocampus. At one-month following injury, AT8 expression was elevated in comparison to sham-injury $(\mathrm{q}=4.34, p<0.05)$ (Figure $3.3 \mathrm{C})$. No significant difference was observed at 7-days post-injury on the ipsilateral side or between any groups or time points on the contralateral side (Figure 3.3 D).

At one-month following repetitive blast exposure, a significant increase phosphorylation recognized by the AT270 antibody was measured in the contralateral hippocampus of rats compared to anesthetized control animals $(\mathrm{q}=4.03, p<0.05)$ (Figure $3.3 \mathrm{~F}$ ). No significant differences were observed in tau phosphorylation recognized by AT270 in either ipsilateral (Figure $3.3 \mathrm{G}$ ) or contralateral (Figure $3.3 \mathrm{H}$ ) hippocampus of mice receiving modified closed-head injury.

Hyperphosphorylation of tau following neurotrauma occurs in perivascular brain regions

Clinical case series documenting patients with CTE have demonstrated the deposition of neurofibrillary tangles (NFTs), an end product of tau hyperphosphorylation, in perivascular regions, which is a distinct difference from other tauopathies such as Alzheimer's disease (AD). Possible mechanisms leading to NFTs in perivascular regions include the disruption of the blood-brain barrier (BBB), leading to punctate microhemorrhages, red blood cell breakdown, oxidative stress, and finally, persistent neuroinflammation. We observed a significant difference in tau hyperphosphorylation within perivascular regions of the contralateral hippocampus in rats exposed to repeat 
blast in comparison to control when identified with both AT8 $(\mathrm{t}=4.46, p<0.001)$ and AT270 $(\mathrm{t}=11.47, p<0.001)$ at 1 -month post-injury (Figure 3.4$)$. These findings were consistent with prior reports of CTE in humans and from our collection of post-mortem human samples with documented CTE.

Neurotrauma is associated with conformational changes in tau that are recognized precursors of neurofibrillary tangle formation

Following hyperphosphorylation, tau is purported to undergo conformational changes associated with subsequent insolubility and deposition/precipitation in the form of neurofibrillary tangles. Following neurotrauma in rats and mice, we found an elevation in markers of conformational change of the tau molecule based on immunoblotting with PHF-1 and CP13. After blast exposure, markers of tau conformational change were observed in the contralateral hemisphere of rats, while after the impact procedure in mice, the markers were found ipsilaterally. No significant differences in markers of tau conformation change were observed in the ipsilateral hippocampus after blast injury for neurofibrillary precursor PHF-1 (Figure 3.5 A) or CP13 (Figure 3.5 E).

A significant difference was observed in PHF-1 $\left(\mathrm{F}_{(2,11)}=7.92, p<0.01\right)$ and CP13 $\left(\mathrm{F}_{(2,9)}=6.03, p<0.05\right)$ levels in the contralateral hippocampus of rats exposed to blast injury. At one month following a single blast, a significant increase in PHF expression was measured compared to control $(\mathrm{q}=4.76, p<0.05)$ (Figure $3.5 \mathrm{~B})$.

A significant difference was observed in PHF-1 $\left(\mathrm{F}_{(2,9)}=7.18, p<0.05\right)$ levels in the ipsilateral hippocampus of mice exposed to injury. At one month following modified 
impact, a significant increase in PHF-1 expression was measured compared to control (q $=5.17, p<0.05$ ) (Figure 3.5 C). No significant differences were observed in PHF-1 expression in the contralateral hippocampus of mice exposed to cortical impact (Figure 3.5 D).

At one month after repetitive blast exposure, a significant increase in CP13 expression was measured in the contralateral hippocampus of rats compared to control (q $=4.73, p<0.05)$ (Figure 3.5 F). A significant difference was observed in $\mathrm{CP} 13\left(\mathrm{~F}_{(2,9)}=\right.$ 21.70, $p<0.001)$ levels in the ipsilateral hippocampus of mice exposed to injury. At one month following impact, a significant increase in CP13 expression was measured compared to control $(q=9.28, p<0.001)$ (Figure $3.5 \mathrm{G})$. No significant differences were observed in CP13 expression in the contralateral hippocampus of mice exposed to cortical impact (Figure $3.5 \mathrm{H}$ ).

Similar findings were seen using immunohistochemistry when comparing repeatinjured animals to sham-injured animals at 1 month post-injury. Specifically, a significant difference was seen on PHF-1 staining $(\mathrm{t}=6.06, p<0.001)$ and CP-13 staining $(\mathrm{t}=3.88$,

$p<0.01$ ) (Figure 3.6). Again, the distribution of the staining was notable for being perivascular in nature, a finding shared across the clinical specimens diagnosed with CTE as seen in figure 3.7. We show that PHF, AT8, and a thioflavin stained neurofibrillary tangle are increased perivascular in human CTE specimens. CP-13 is increased in a perivascular distribution following repeat blast in a rat.

\section{Neurotrauma produces cognitive impairments}


Cognitive deficits have long been associated with the neuropathological diagnosis of CTE in the clinical population, particularly amongst the population diagnosed with CTE at a later age in life. Similarly, cognitive impairments in both learning and memory have been associated with neurotrauma but have not been presented in the context of a corresponding tauopathy in both a single and repeat blast injury paradigm. In cohorts of animals subjected to either sham-, single-, or repetitive-injury, spatial acquisition (learning) was assessed 3 weeks after the injury in single-injury animals and the final injury in repeat-injury paradigms (Days 21-27 post-injury). Blast injury exposure was associated with worsened performance in the Morris water maze as evident by latency to find the platform in seconds when analyzed using a two-way repeated measures ANOVA. Post-hoc tests revealed significant differences between anesthetized controls and single-injury animals during spatial acquisition on Days 22-24, and Day 26 (Day 21: $\mathrm{t}=1.38, p>0.05$; Day 22: $\mathrm{t}=3.70, p<0.01 ;$ Day 23: $\mathrm{t}=4.12, \mathrm{p}<0.001 ;$ Day 24: $\mathrm{t}=$ 4.38, $p<0.001$; Day 25: $\mathrm{t}=2.17, p>0.05$; Day 26: $\mathrm{t}=3.20, p<0.01)$. Similarly, posthoc tests demonstrated a significant impairment in acquisition between anesthetized controls and repetitively injured animals on Days 22-26 (Day 21: $\mathrm{t}=0.66, p>0.05$; Day 22: $\mathrm{t}=5.01, p<0.001 ;$ Day 23: $\mathrm{t}=4.16, p<0.001 ;$ Day 24: $\mathrm{t}=4.72, p<0.001 ;$ Day 25: $\mathrm{t}$ $=3.69, p<0.01$; Day 26: $\mathrm{t}=3.76, p<0.01)$. Notably, no differences were seen between single- and repeat-injured animals during the course of acquisition trials, despite the notable difference in tau pathology at this time presented earlier (Figure 3.8). The differences in latency to platform between blast-injured animals and anesthetized control animals is apparent as well based on visualization of the track plots recorded during data acquisition (Figure 3.9). Clearly different swimming patterns emerge with blast-injured 
animals exhibiting what appears to be more thigmotaxis (circling around the outer edge of the pool) than anesthetized control animals that reached statistical significance between controls and repeat-injured animals on Day $23(\mathrm{q}=3.58, p<0.05)$, although this data point did not reach significance on Days 21 and 26 based on automated measurements generated using AnyMaze ${ }^{\mathrm{TM}}$ (Figure 3.8).

Memory, as measured during the probe test (time spent in area surrounding the now-removed platform) conducted on Day 27, again demonstrated deficits in animals subjected to blast-induced neurotrauma when analyzed using a one-way ANOVA $\left(\mathrm{F}_{2,29}=\right.$ 20.01, $p<0.0001)$. Post-hoc tests showed significant differences between sham and single injury animals $(\mathrm{t}=6.44, p<0.05)$ as well as between sham and repetitively injured animals $(\mathrm{t}=8.00, p<0.05)$ but no difference between single injury and repeat injury paradigms (Figure 3.8). These findings were confirmed visually using track plots generated during data acquisition (Figure 3.9). Anesthetized control animals exhibit a greater preponderance of pool crossings and swimming behavior within the region near the platform in contrast to blast-injured animals (Figure 3.9).

\section{Impulsivity is increased following neurotrauma}

Clinically, impulsive behavior has been described extensively in those with a history of repetitive neurotrauma and the context of CTE. To make an analogous comparison between our animal model of blast-induced traumatic brain injury and clinically reported symptoms, the elevated-plus maze was utilized to measure impulsivelike behaviors based upon the percentage of time spent in the open-arms of the maze. Significant differences between groups were observed when analyzed using a one-way 
ANOVA $\left(\mathrm{F}_{2,32}=5.03, p<0.05\right)$. Post-hoc tests revealed a significant difference between sham and single injury animals $(\mathrm{q}=3.53, p<0.05)$ as well as between sham and repetitively injured animals $(\mathrm{q}=4.21, p<0.05)$. No difference was seen between single and repeat injury paradigms with regards to percentage of time spent in the open arms of the apparatus (Figure 3.10). While not statistically significant, a trend towards a greater distance traveled in the open arms was observed with increasing levels of neurotrauma, evidence consistent with the percentage of time spent in the open arms (Figure 3.10). Conversely, a trend was also present regarding distance traveled in the closed arms with animals subjected to neurotrauma traveling less distance (Figure 3.10). Post-hoc tests revealed no statistical significance when comparing sham to single injury groups but significance was reached when comparing sham to repetitively-injured animals $\left(\mathrm{F}_{2,33}=\right.$ $3.06 ; \mathrm{q}=3.47, p<0.05)$.

\section{DISCUSSION}

While the clinical literature has been inundated with reports of CTE in athletes and soldiers alike, few experimental tools exist for investigating disease pathophysiology, establishing diagnostic criteria, and discovering preventative or therapeutic agents. For these reasons, preclinical models of CTE are highly desirable. In this work we demonstrate two preclinical models capable of generating some biochemical and behavioral hallmarks of CTE, namely tauopathy and impulsive-like behavior. Furthermore, this work illustrates the utility of two distinct models and injury paradigms, namely blast exposure versus a more traditional modified closed-head injury, and repeat versus single injury paradigms respectively. Likewise, the fact that this work was

completed in two different species and builds upon prior work by both West Virginia 
University and the University of Rochester laboratories validates the use of both mice and rats in the preclinical modeling of neurotrauma related neurodegeneration (Logsdon et al 2014, Petraglia et al 2014b, Petraglia et al 2014c). The modified closed-head injury represents a helmeted design with controlled placement of the impact likely accounting for ipsilateral deficit. The tauopathy development period following modified controlled impact was not previously elucidated therefore warranting the 7-day time point. Petraglia and colleagues show that astrocyte activation and cell death occurs at 7 days post modified controlled impact (Petraglia et al 2014c). We previously reported that tauopathy following blast in rats does not develop until weeks after injury and is on the contralateral side due to coup/contra-coup injuries (Lucke-Wold et al 2015d).

The potential utility of these, as well as other preclinical models of neurotrauma related neurodegeneration, is highly promising for investigation of disease pathophysiology, particularly as related to biochemical endpoints associated with CTE. To fully develop a CTE model, transgenic rodents will be needed that include amyloid, tau, and TDP43 pathology. The quest for elucidating the underlying mechanisms behind CTE development is ongoing. In previous work, we have shown blast causes substantial blood brain barrier disruption (Lucke-Wold et al 2014a), endoplasmic reticulum stress activation (Logsdon et al 2014), and oxidative stress (Lucke-Wold et al 2015a). We show in this work the induction of hyperphosphorylated tau, conformational changes in tau, and more advanced precursors of NFT formation with the usage of CP13 and PHF-1 antibodies. AT8 binds to serine 199 and 202 as well as threonine 205. AT270 binds to threonine 181, PHF binds to serine 396 and 404, and CP13 binds to serine 202. Serine 396 and 202 are only exposed after tau undergoes conformational change (Lucke-Wold et 
al 2014c). These changes are the ultimate result of tau hyperphosphorylation and protein misfolding/aggregation, and are likely related to dysregulation of the tau kinase/phosphatase system. Peclinical modeling of neurotrauma related neurodegeneration will allow for the elucidation of how and when these kinases and phosphatases become dysregulated, and will allow for increased understanding of the disease process. Additionally, these studies will provide targets for direct or indirect therapeutic development. Similarly, preclinical models may prove instrumental in identification of diagnostic and prognostic tests for establishing a diagnosis of CTE and tracking disease progression. There is a clinical need for a rapid, cheap, and reliable diagnostic test to predict severity of CTE. Currently neuropathological examination remains the gold standard for diagnosis with some studies purporting the use of PETbased imaging for diagnosis (Gandy \& DeKosky 2014, Mitsis et al 2014, Small et al 2013). It is possible that development and validation of these techniques, and others such as diffusion tensor imaging and magnetic resonance spectroscopy may be accelerated through the application to preclinical models. The current limitation is that advanced imaging is expensive, cumbersome, and requires expertise from subspecialty radiologists.

Despite this work illustrating what we believe represents a clear step forward in the study of neurotrauma related neurodegeneration, it is clear that further study is warranted. Pre-clinical models must be utilized to more fully characterize behavioral, biochemical, imaging, and electrophysiological/functional changes associated with the development of CTE. Future studies will likely address the development of behavioral/functional deficits in relation to biochemical changes temporally and assess the chronicity of changes based on the number, severity, and inter-injury interval of 
neurotrauma-related events. The development of better transgenic models is critical as the field moves forward. Performing studies such as these will allow for the questions raised in our accompanying review be addressed. Specifically, what is the role of the 1) inter-

injury interval, 2) number of impacts, 3) impact severity, 4) age at time of impacts, 5) mechanism of impact, 6) genetics, 7) gender, and 8) effect of environment on the likelihood and/or progression of CTE development.

\section{ACKNOWLEDGEMENTS}

A West Virginia University Research Funding Development grant funded the project. An American Foundation of Pharmaceutical Education Pre-doctoral Fellowship supported Brandon Lucke-Wold and Aric Logsdon. An American Medical Association Foundation Seed Grant and a Neurosurgery Research and Education Foundation Medical Student Summer Research Fellowship supported Brandon Lucke-Wold. 

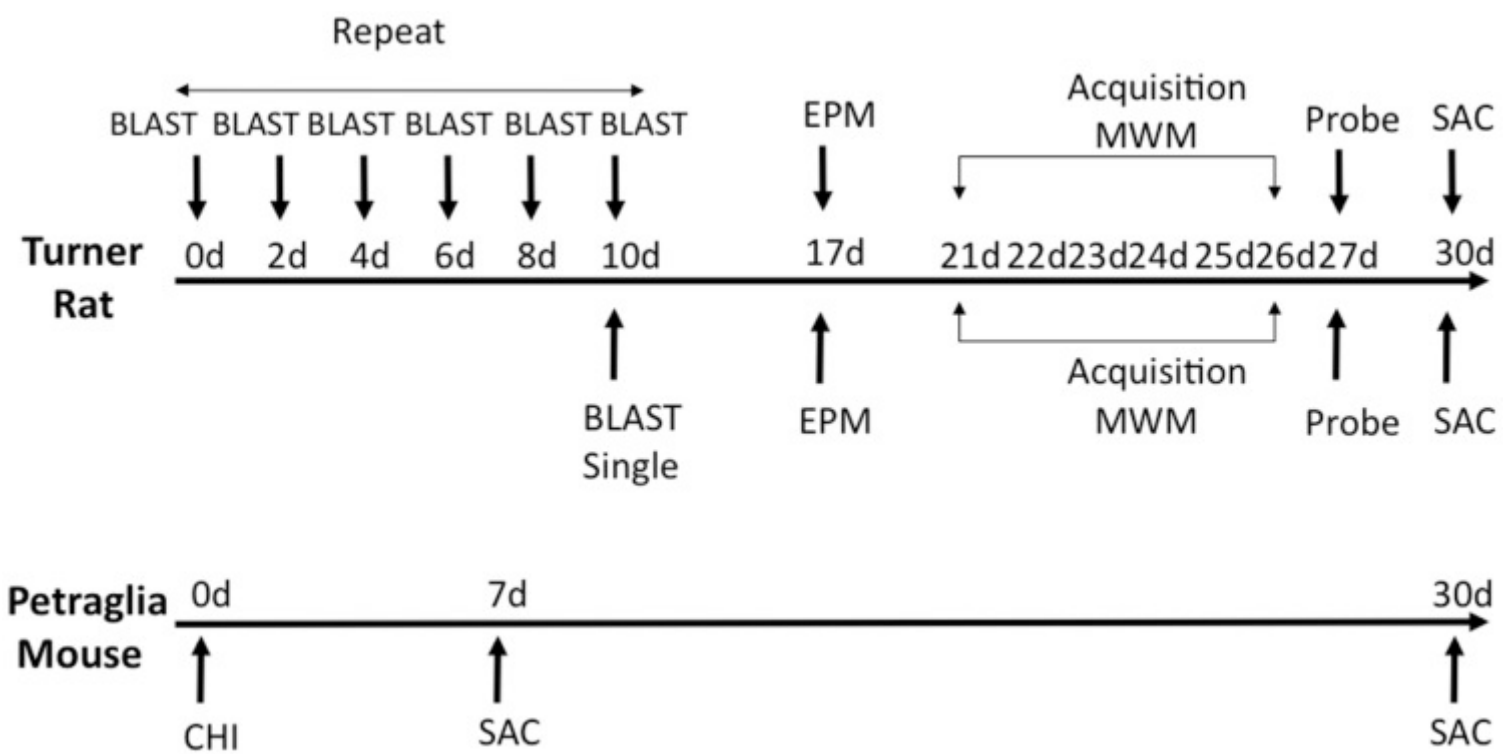

Figure 3.1: Schematic showing experimental design and behavioral experiments. Time of sacrifice for biochemical experiments is also shown. 


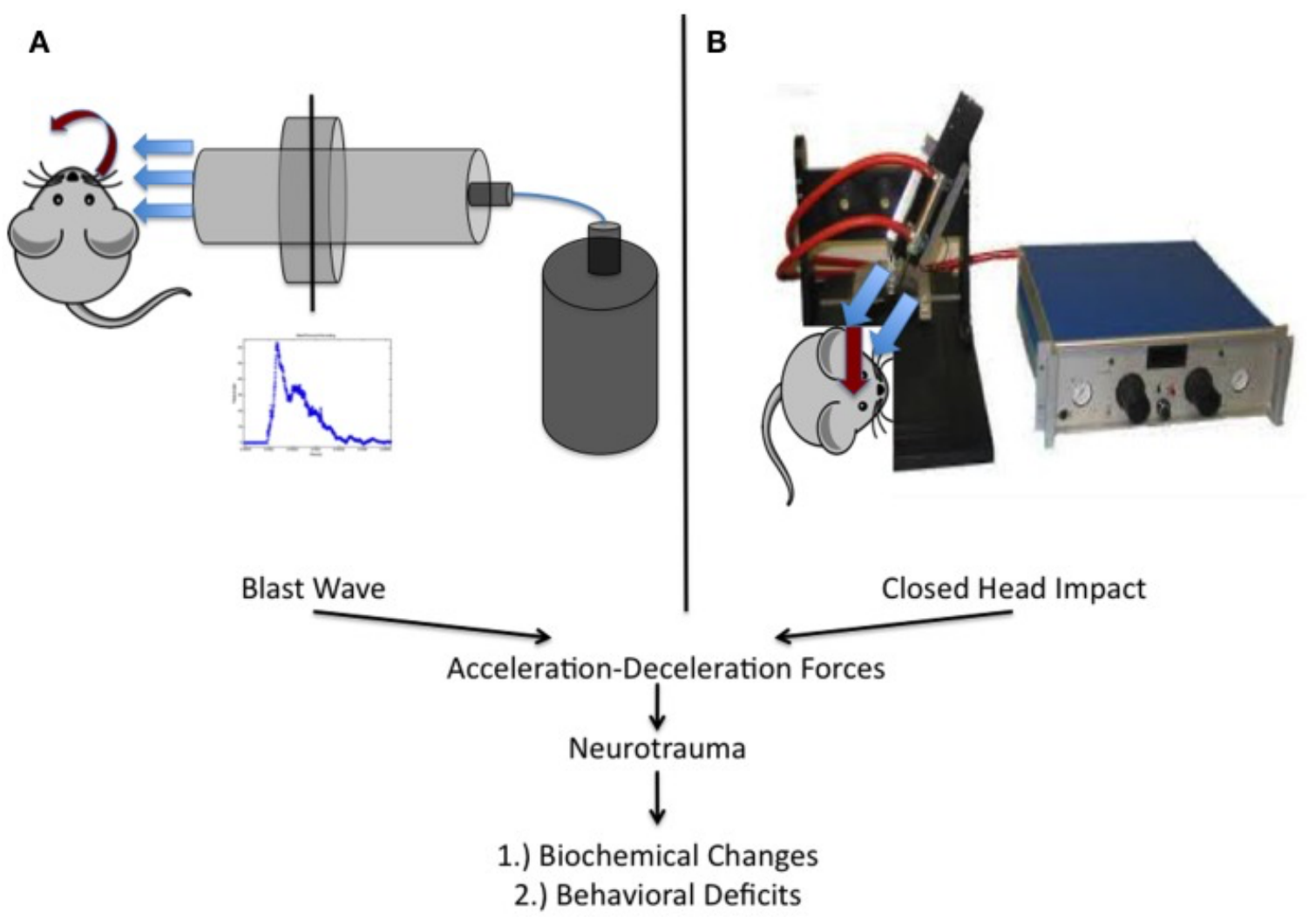

Figure 3.2: Schematic representation of the two injury models utilized within this work.

(A) Experimental setup utilized at West Virginia University for the study of blast-induced neurotrauma. The shock tube consists of a high-pressure driver section (nitrogen gas filled) and a low-pressure driven section (ambient air filled). When the membrane dividing the chambers ruptures, the blast wave is formed and released, encountering the rat from the right side of the cranium. (B) Depiction of the model utilized at the University of Rochester in un-anesthetized mice. An electromagnetic impactor used in CCI was modified with a rubber tip and a specially designed helmet was placed on the mouse as previously described. 


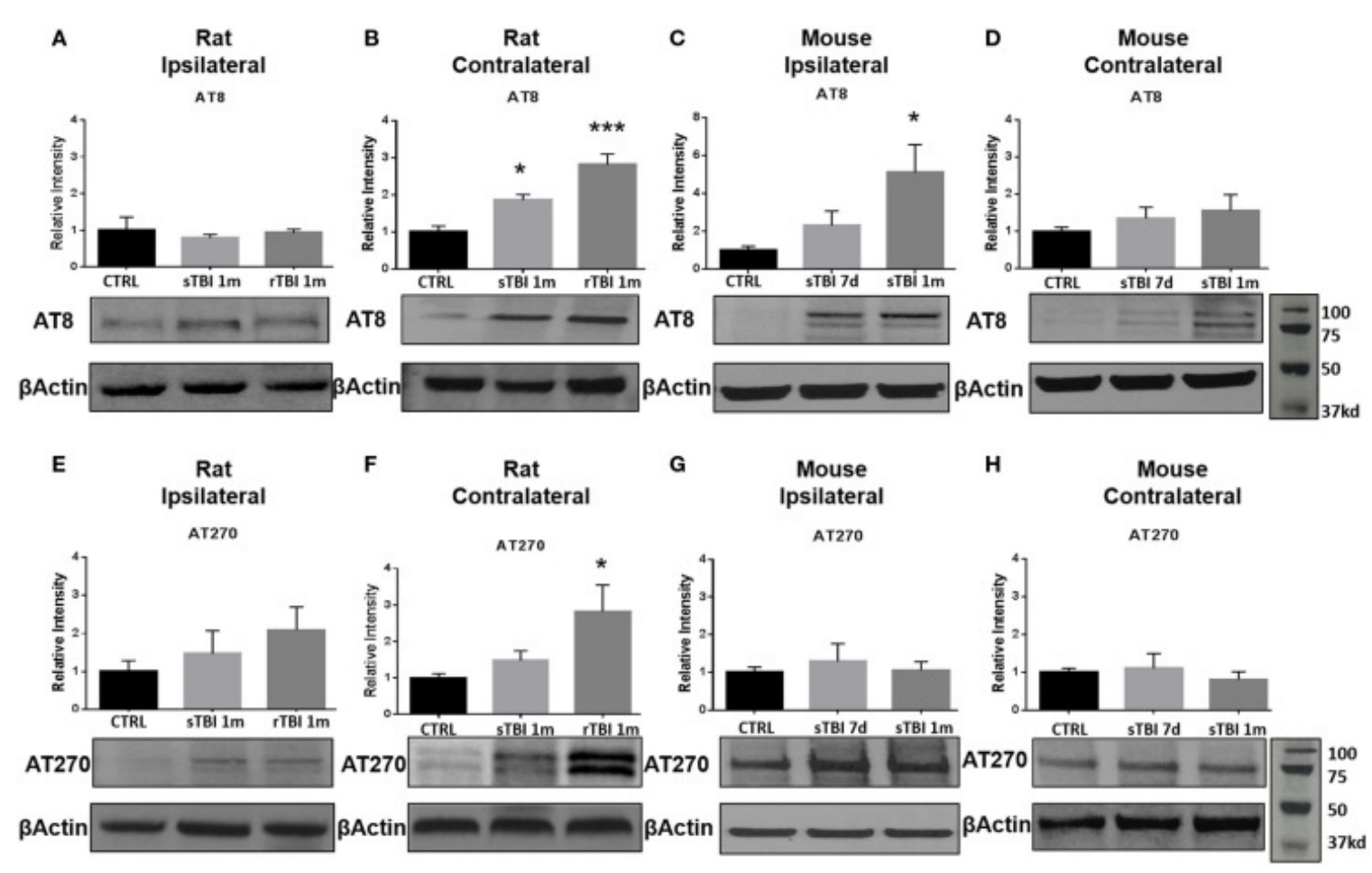

Figure 3.3: Blast and closed-head injury models both induced tau hyperphosphorylation.

(A) Immunoblots show no significant difference in tau phosphorylation at serine sites 199/202 and threonine site 205 (AT8) at one month after single and repeat blast exposures in the ipsilateral rat hippocampus. (B) A significant increase in AT8 expression was measured after single ( ${ }^{*} p<0.05$ vs. CTRL) and repeat blast exposure $(* * * p<0.001$ vs. CTRL) in the contralateral rat hippocampus. (C) AT8 expression was significantly increased at one month after closed-head injury in the ipsilateral mouse hippocampus $(* p<0.05$ vs. CTRL). (D) No significant differences were observed in AT8 expression in the contralateral mouse hippocampus. (E) Immunoblots show no significant difference in tau phosphorylation at threonine site 181 (AT270) at one month after single and repeat blast exposure in the ipsilateral rat hippocampus. (F) A significant increase in AT270 expression was measured at one month after repeat blast exposure $\left({ }^{*} p<0.05\right.$ vs. CTRL) in the contralateral rat hippocampus. (G) No significant differences 
were observed in AT270 expression in the ipsilateral mouse hippocampus, $(\mathbf{H})$ or the contralateral mouse hippocampus after closed-head injury. One-way ANOVA Tukey's post-hoc analysis (values represent mean \pm s.e.m.; normalized to $\beta$-actin) $(n=3-5)$. 

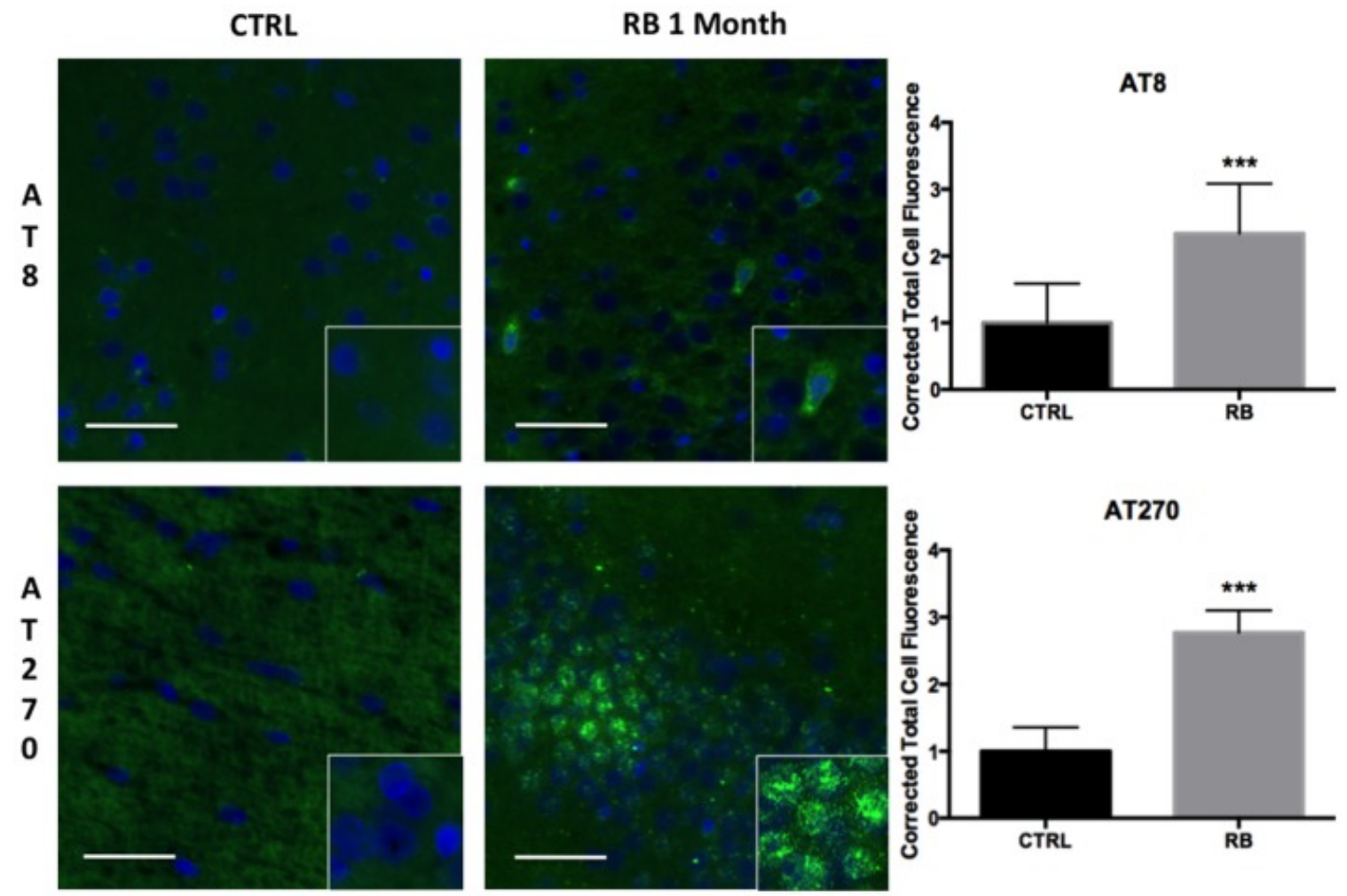

Figure 3.4: Tau hyperphosphorylation is seen in microfoci throughout the contralateral superficial cortex. AT8 was significantly increased at 1 month post repetitive blast $(\mathrm{t}=$ $4.455, p<0.001)$. AT270 was also significantly increased following repetitive blast $(\mathrm{t}=$ $11.47, p<0.001)$. AT270 was increased in a circular distribution. Tau hyperphosphorylation is an indicator of progressive pathology. Values were calculated using a student's t-test comparing the mean difference between groups. 


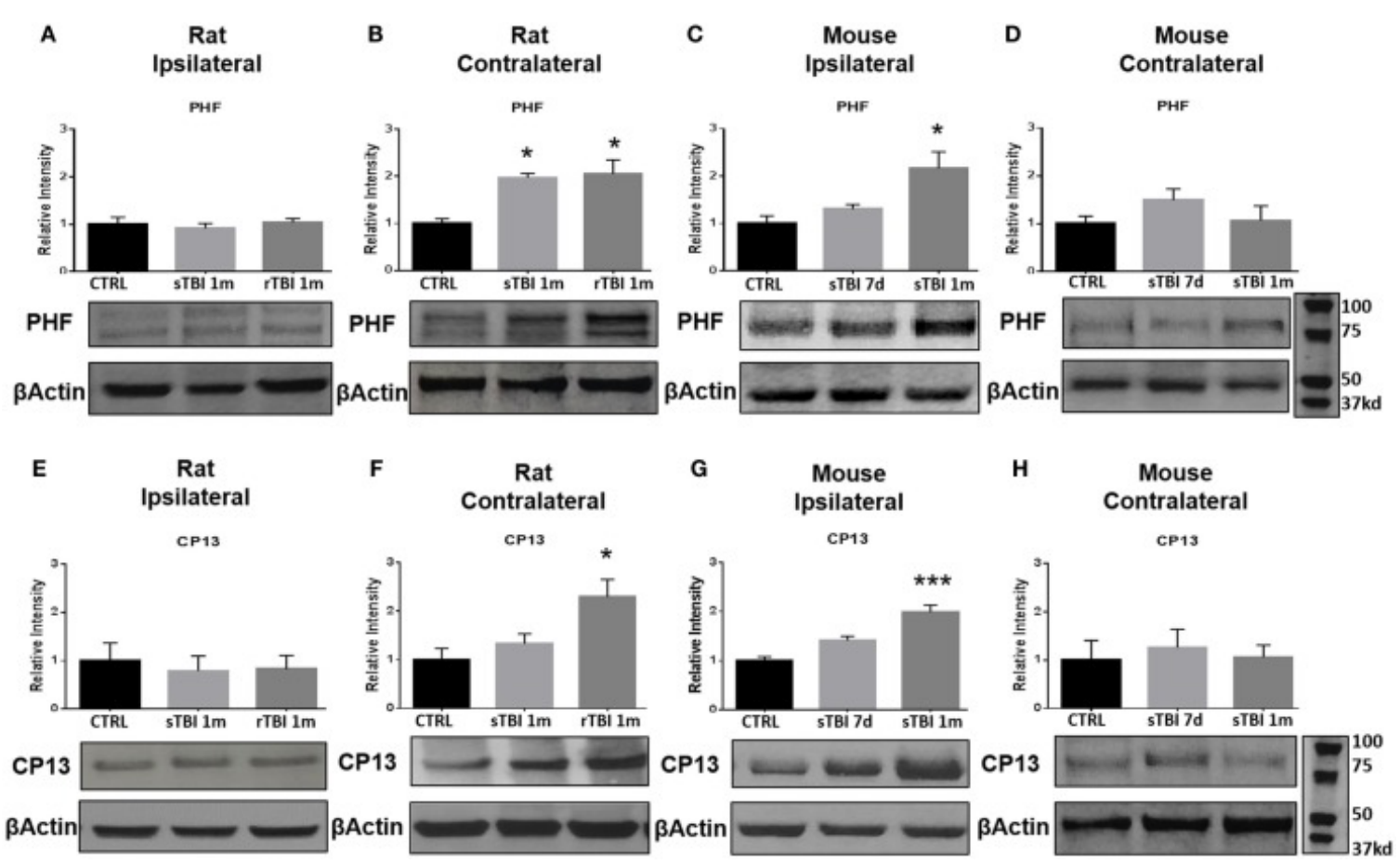

Figure 3.5: Blast and closed-head injury models both induce conformational changes in tau proteins. (A) Immunoblots show no significant difference in markers of tau conformational change at serine site 396/404 (PHF) at one month after single and repeat blast exposure in the ipsilateral rat hippocampus. (B) A significant increase in PHF expression was measured after single ( $* p<0.05$ vs. CTRL) and repeat blast exposure $\left({ }^{*} p<0.05\right.$ vs. CTRL) in the contralateral rat hippocampus. (C) PHF expression was significantly increased at one month after closed-head injury in the ipsilateral mouse hippocampus. (D) No significant differences were observed in PHF expression in the contralateral mouse hippocampus. (E) Immunoblots show no significant difference in markers of tau conformational change at serine site 202 (CP13) at one month after single and repeat blast exposure in the ipsilateral rat hippocampus. (F) A significant increase in CP13 expression was measured at one month after repeat blast exposure $\left({ }^{*} p<0.05\right.$ vs. CTRL) in the contralateral rat hippocampus. (G) A significant increase in CP13 
expression was measured at one month after closed-head injury in the ipsilateral mouse hippocampus $(* * * p<0.001$ vs. CTRL). (H) No significant differences were observed in CP13 expression in the contralateral mouse hippocampus after closed-head injury. Oneway ANOVA Tukey's post-hoc analysis (values represent mean \pm s.e.m.; normalized to $\beta-\operatorname{actin})(n=3-5)$. 

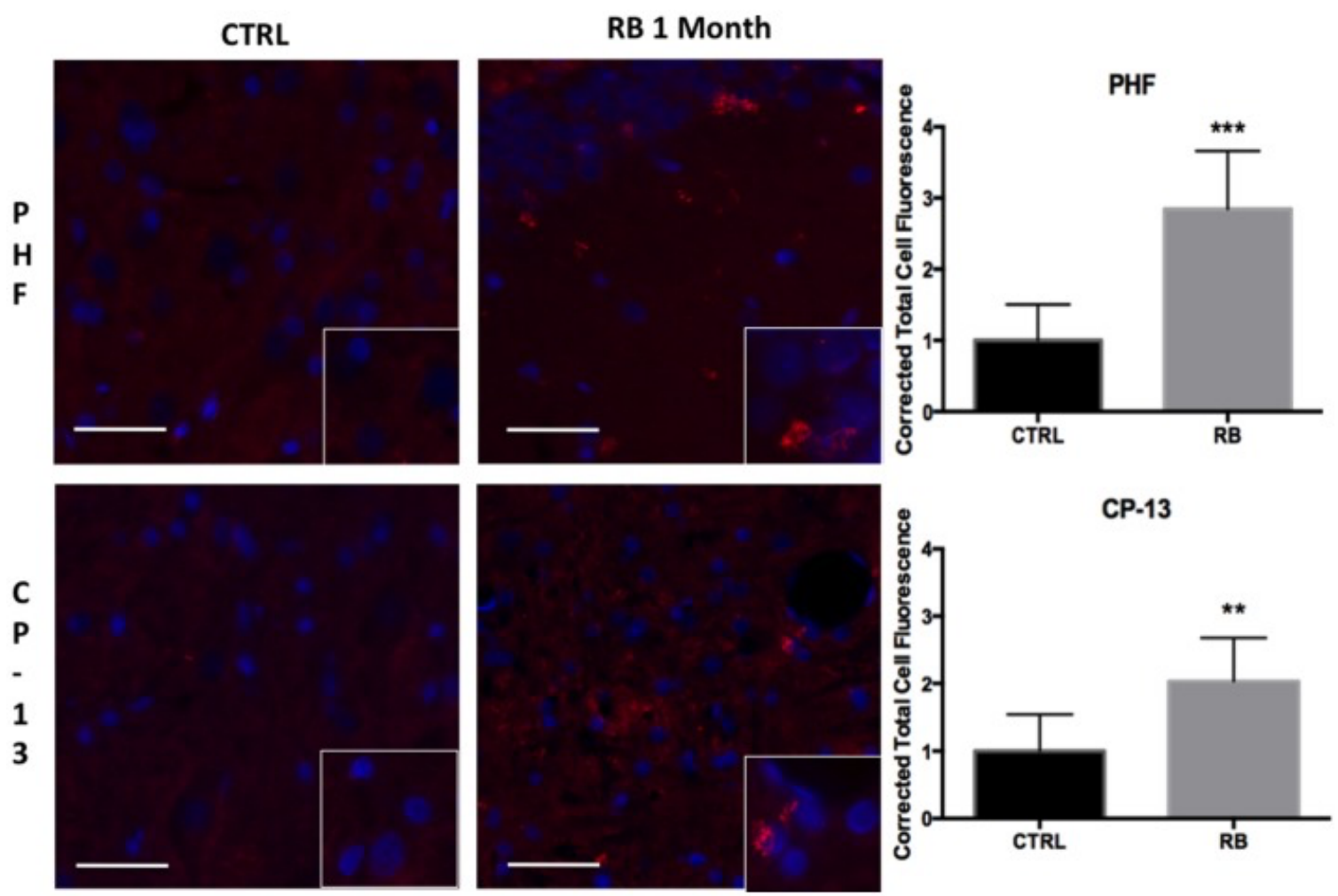

Figure 3.6: Tau conformational markers were increased throughout the contralateral superficial cortex. PHF was significantly increased at 1 -month post repetitive blast $(\mathrm{t}=$ $6.055, p<0.001)$. CP-13 staining was also significantly increased following repetitive blast $(\mathrm{t}=3.883, p<0.01)$. CP-13 was increased in a perivascular distribution. Tau conformational change is required for the formation of neurofibrillary tangles. Values were calculated using a student's t-test comparing the mean difference between groups. 


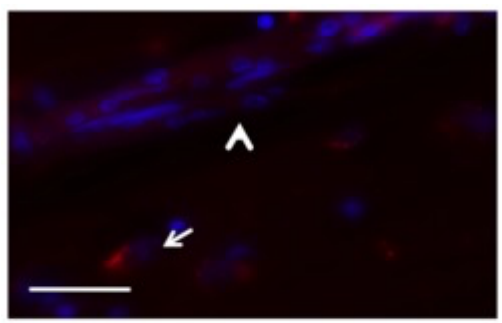

Human PHF

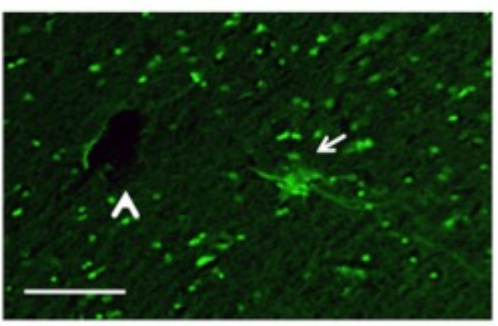

Human NFT

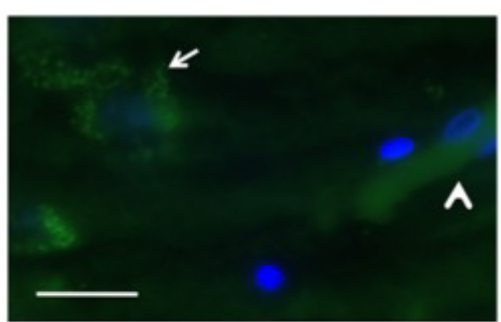

Human AT8
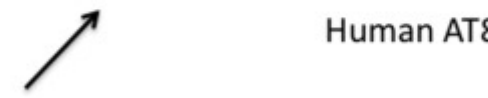

\section{Perivascular}

Tau
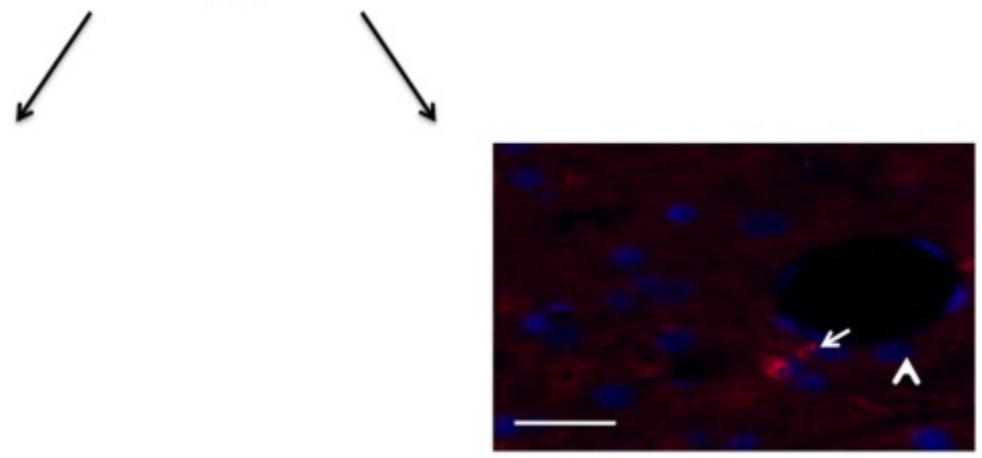

Rat CP-13

Figure 3.7: Tauopathy is seen in a perivascular distribution. PHF and AT8 were increased adjacent to longitudinal microvessels in human patients diagnosed with chronic traumatic encephalopathy. A neurofibrillary tangle stained with thioflavin was seen adjacent to a cut vessel lumen in the brain of a retired professional football player. Similarly, CP-13 was increased next to a cut vessel lumen in the contralateral cortex 1 month following repeat blast in the rat. Arrows indicate tauopathy while arrowheads point out vessels. 


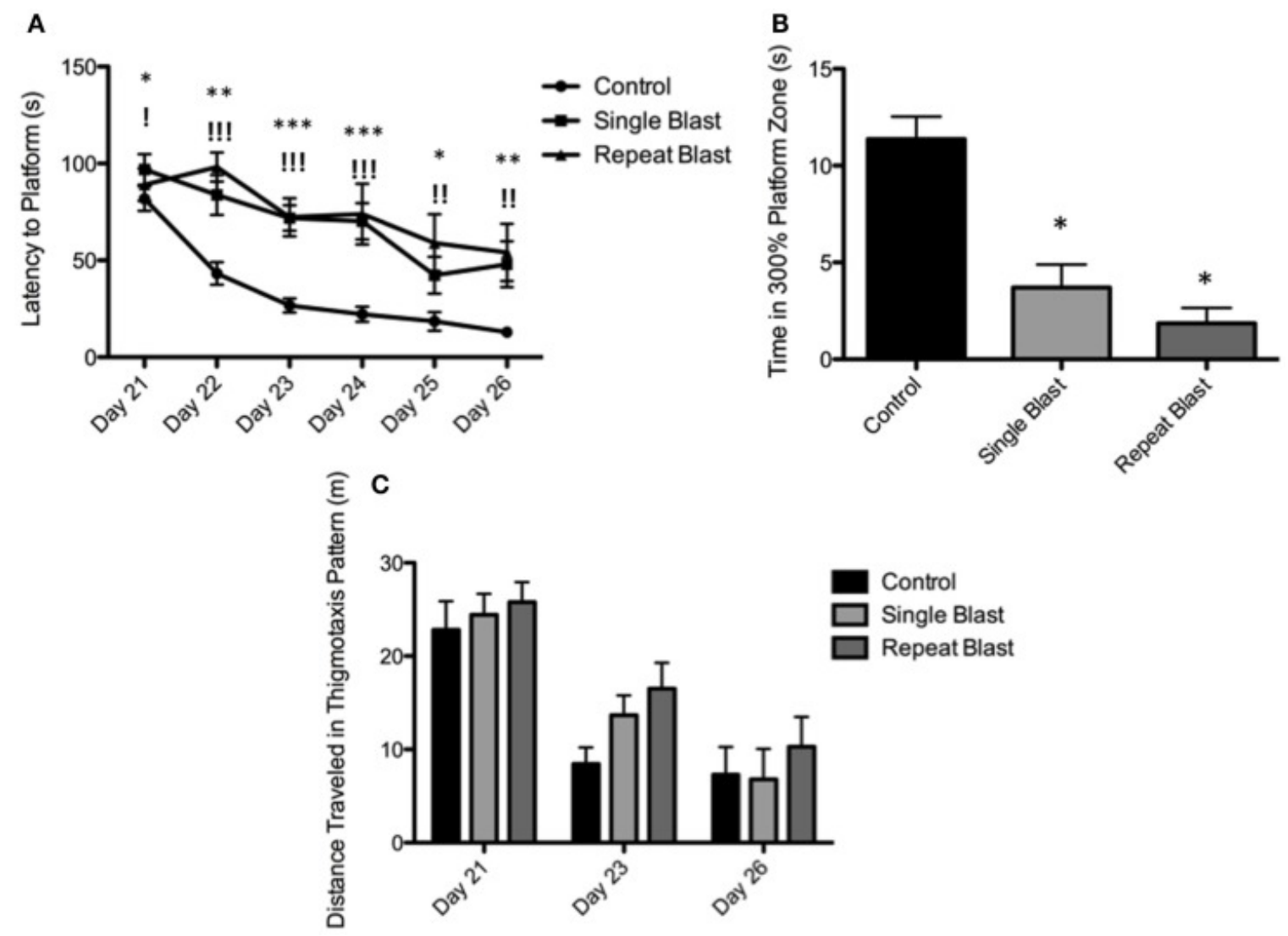

Figure 3.8: Blast-induced brain injury produces deficits in learning and memory in rats.

(A) Spatial acquisition trials conducted on Days 21-26 post-injury revealed deficits in learning in single-injury animals on Days 22-24 and 26 in comparison to control.

Repeat-injury was associated with deficits on Days 22-26 in comparison to control. No differences were seen between single- and repeat-injury paradigms. (B) The probe test on Day 27 revealed a statistically significant difference in time spent near the prior platform location between injured animals (single or repeat) and control animals. No difference was seen between single- and repetitively-injured rats. (C) Animals exposed to neurotrauma exhibited a trend towards increased thigmotaxis during spatial acquisition procedures but this finding did not reach statistical significance. 

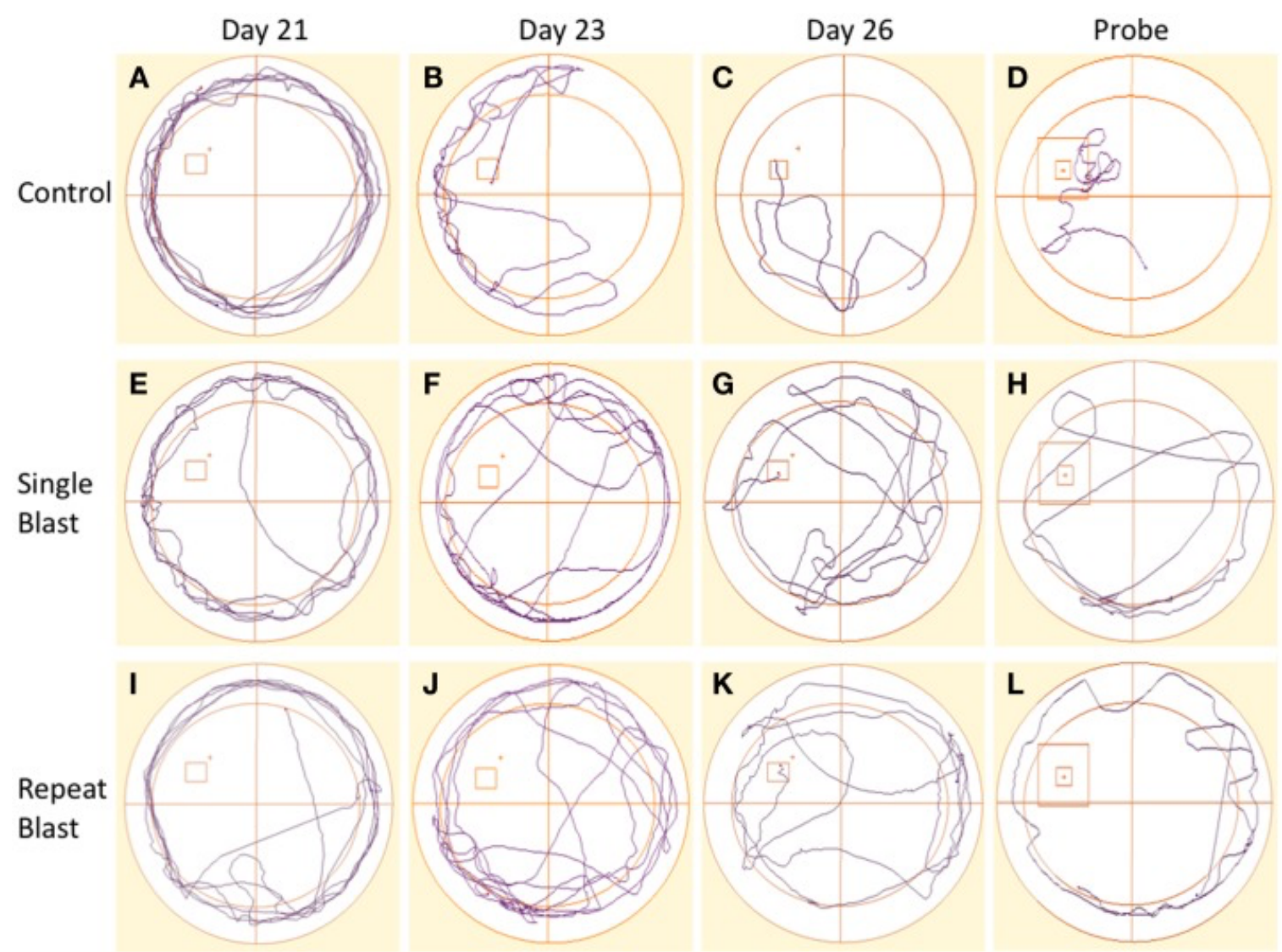

Figure 3.9: Track plots acquired across spatial acquisition and probe testing in rats. (AD) Track plots generated from anesthetized control animals on Day 21, Day 23, Day 26, and during the probe test. (E-H) Track plots generated from single-injured animals on Day 21, Day 23, Day 26, and during the probe test post-injury. (I-L) Track plots generated from repetitively-injured animals on Day 21, Day 23, Day 26, and during the probe test post-injury. Injured animals appear to spend more time exhibiting thigmotaxis behaviors (circling edge of pool) on later days and in the probe trial fail to spend as much time as the anesthetized control animals within the probe region and the direct area surrounding the probe region. 

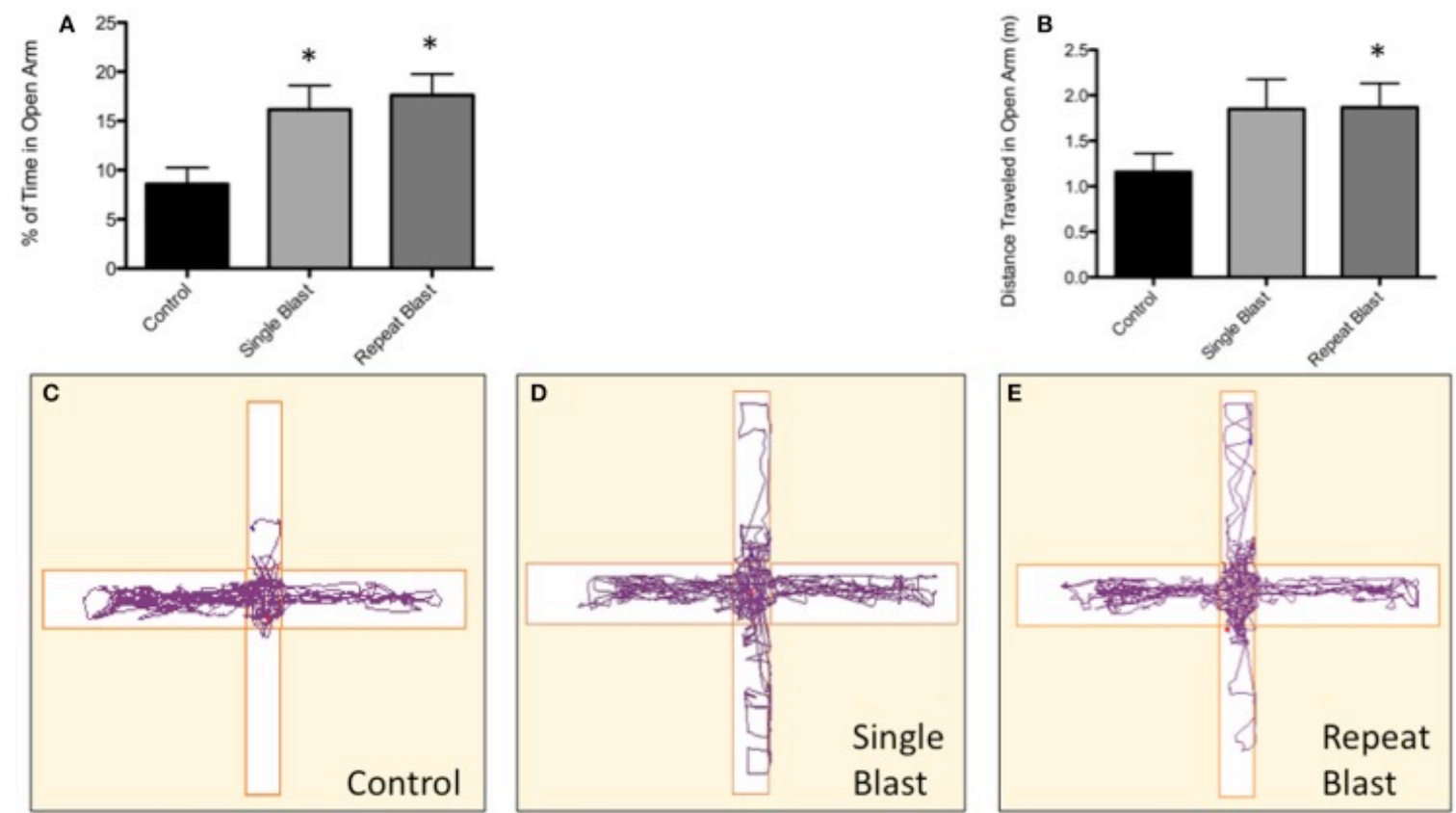

Figure 3.10: Blast-induced brain injury in rats produces an increase in impulsive behavior based on the elevated-plus maze. (A) A significant difference ( $p<0.05$ vs. CTRL) was seen between anesthetized control animals and both single and repeat blasts with regards to the percentage of time spent in the open arm of the elevated-plus maze. No difference was measured between single and repeat-injury paradigms. (B) While not reaching statistical significance, a trend towards greater distance traveled in open arms was seen with neurotrauma, a finding consistent with the documented increase in time spent in the open arms. (C) Animals subjected to neurotrauma appeared to travel less distance in the closed arms of the maze, although this finding did not reach statistical significance. (D) Track plot showing little exploration of the open arm by control animals. (E) Track plot showing increased exploration of the open arms of the elevatedplus maze by single-injured animals. (F) Track plot showing increased exploration of the open arms of the elevated-plus maze by repetitively injured animals. 


\section{Chapter Four}

Bryostatin-1 Restores Blood Brain Barrier Integrity following Blast-Induced Traumatic Brain Injury

This work is published in Mol Neurobiol. 2015 Dec;52(3):1119-34. doi: 10.1007/s12035014-8902-7. Epub 2014 Oct 10. PMID: 25301233

Lucke-Wold BP, Logsdon AF, Smith KE, Turner RC, Alkon DL, Tan Z, Naser ZJ, Knotts CM, Huber JD, Rosen CL 


\section{ABSTRACT}

Recent wars in Iraq and Afghanistan have accounted for an estimated 270,000 blast exposures amongst military personnel. Blast traumatic brain injury (TBI) is the 'signature injury' of modern warfare. Blood brain barrier (BBB) disruption following blast TBI can lead to long-term and diffuse neuroinflammation. In this study, we investigate for the first time the role of bryostatin-1, a specific protein kinase $\mathrm{C}$ (PKC) modulator, in ameliorating BBB breakdown. 37 Sprague dawley rats were used for this study. We utilized a clinically relevant and validated blast model to expose animals to moderate blast exposure. Groups included: control, single blast exposure, and single blast exposure + bryostatin-1. Bryostatin-1 was administered i.p. $2.5 \mathrm{mg} / \mathrm{kg}$ after blast exposure. Evan's blue, immunohistochemistry, and western blot analysis were performed to assess injury. Evan's blue binds to albumin and is a marker for BBB disruption. Single blast exposure caused an increase in permeability compared to control $(\mathrm{t}=4.808, \mathrm{p}<0.05)$, and a reduction back towards control levels when bryostatin-1 was administered $(\mathrm{t}=5.113, \mathrm{p}<0.01)$. Three important PKC isozymes, PKC $\alpha, \delta$, and $\varepsilon$, were co-localized primarily with endothelial cells but not astrocytes. Bryostatin-1 administration reduced toxic PKC $\alpha$ levels back towards control levels ( $\mathrm{t}=4.559, \mathrm{p}<0.01)$, and increased the neuroprotective isozyme $\mathrm{PKC} \varepsilon(\mathrm{t}=6.102, \mathrm{p}<0.01)$. Bryostatin-1 caused a significant increase in the tight junction proteins VE-cadherin, ZO-1, and occludin through modulation of PKC activity. Bryostatin-1 ultimately decreased BBB breakdown potentially due to modulation of PKC isozymes. Future work will examine the role of bryostatin-1 in preventing chronic neurodegeneration following repetitive neurotrauma. 
KEY WORDS: Blood brain barrier, bryostatin-1, protein kinase $C$, tight junction proteins

\section{INTRODUCTION}

1.7 million Americans experience a traumatic brain injury (TBI) each year in the United States (Kou \& Vandevord 2014). Over 70\% of those patients sustain some form of disability following TBI (Schneider et al 2014). The mechanisms by which TBI contributes to permanent homeostatic changes within the central nervous system (CNS) remain poorly understood. Recent evidence implicates disruption of the blood brain barrier (BBB) as an important indicator of brain injury (Puvenna et al 2014). Blast TBI, in particular, contributes to a vascular pressure surge that challenges BBB integrity (Simard et al 2014). Blast TBI also causes significant alterations in ZO-1, occludin, and VE-cadherin, important tight junction proteins (Hue et al 2014). The disruption is not universal across vasculature however, with scattered lesions spread across the brain at specific microfoci (Yeoh et al 2013). Oxidative stress from damaged tissue causes increased BBB permeability at these microfoci (Abdul-Muneer et al 2013). The resulting BBB dysfunction contributes to inflammatory cascades and injury expansion (Perez-Polo et al 2013). The increase in blast-induced BBB permeability is transient with a return of tight junction function within 3 days, but the subsequent injury response is long-lasting (Readnower et al 2010).

One possible reason for these long-lasting effects is changes in protein kinase $\mathrm{C}$ (PKC) isozyme activity after injury. Some $\mathrm{PKC}$ isozymes, $\mathrm{PKC} \alpha, \beta$, and $\varepsilon$, translocate to the plasma membrane within 3 hours after TBI and remain active for days (Muscella et al 
2008, Padmaperuma et al 1996, Yang et al 1993). PKC $\alpha$ activity has been linked to neuronal apoptosis following TBI (Chen et al 2012), and is closely tied with glutamate receptor signaling (Luo et al 2014b). PKC $\alpha$ and $\delta$ activity can cause an uncoupling of NMDA receptors from spectrin mediated through sigma-1 receptor activation leading to calcium oscillations (Geddes-Klein et al 2006, Giordano et al 2005, Roh et al 2010). The calcium oscillations contribute to mitochondrial dysfunction and cell death (Pandya et al 2013). PKC $\alpha$ and $\delta$ can also hyperphosphorylate structural proteins such as tau and TBI61 within the hippocampus following injury (Yang et al 1994). Interestingly, PKC activity also decreases cerebral edema following TBI (Karasu et al 2010, Yang et al 1993). Three important isozymes play important roles in cerebral vasculature. PKCa regulates ZO-1 and occludin tight junction proteins (Kim et al 2010). PKC $\delta$ activity regulates vessel constriction and vascular tone (Kizub et al 2014). PKC $\varepsilon$ decreases vessel tone and provides neuroprotection (Lin et al 2012). Despite these associations, the role of specific PKC isozymes in BBB disruption following blast TBI has yet to be elucidated.

Although PKC activity has not been well studied in TBI, available evidence suggests that PKC isozymes play an important role in neural injury. An $80 \%$ increase in PKC activity was reported 3 hours after TBI and contributes to secondary neuronal injury (Yang et al 1993). Compounds that modulate PKC isozymes therefore warrant further investigation. The potent PKC modulator, bryostatin-1, has profound neuroprotective effects in animal models of neural injury. The single study using bryostatin-1 for treatment of mild TBI showed profound protection against learning and memory deficits in mice by increasing the $\alpha$-secretase, ADAM10 (Zohar et al 2011). Although PKC modulation has not been extensively studied in TBI models, in an Alzheimer's disease 
model it increased $\mathrm{PKC} \varepsilon$, which facilitated amyloid $\beta$ plaque degradation by increasing the enzyme neprilysin (Lim \& Alkon 2014). It also enhanced gabaergic signaling improving learning and memory through PKC $\alpha$ modulation and decreased synaptic loss (Hongpaisan et al 2011, Xu et al 2014). Following ischemic stroke, bryostatin-1 modulated PKC $\alpha$ and $\varepsilon$ to enhance survival and reduce edema in aged Sprague-Dawley rats (Tan et al 2013). Although Alzheimer's disease and stroke are vastly different than TBI, they do share similar secondary mechanisms of injury, such as PKC and microglia activation, that should be carefully investigated

Herein, we show for the first time that PKC isozymes are increased after blastinduced TBI, and are correlated with acute BBB breakdown and tight junction protein changes. Using our clinically relevant and validated blast model (Turner et al 2013), we observed statistically significant acute changes in BBB permeability 6 hours after single blast exposure. Interestingly, the PKC modulator bryostatin-1 reduced BBB permeability, decreased levels of the detrimental PKC $\alpha$ isozyme, and increased the beneficial PKCE isozyme. The mechanism of bryostatin-1's beneficial effects is closely linked to regulation of tight junction proteins through specific modulation of PKC isozymes. Occludin closely interacts with the scaffolding proteins ZO-1 and VE-Cadherin (Morrow et al 2010), and our data show that bryostatin-1 significantly elevated levels of occludin, VE-Cadherin, and ZO-1. Increasing the levels of tight junction proteins has been shown to help maintain BBB integrity (Goncalves et al 2012). Modulation of PKC expression may therefore be a promising treatment strategy for preventing BBB disruption following acute neurotrauma.

\section{METHODS}




\section{Animals and treatments}

Thirty-seven (37) young adult (300-350g) male Sprague Dawley rats (Hilltop Lab Animals, Inc.) were used for this study. Animals were housed in pairs with $12 \mathrm{~h}: 12 \mathrm{~h}$ dark to light cycle and food available ad libitum. The Institutional Animal Care and Use Committee of West Virginia University approved all procedures and experiments involving animals. The experiments were performed in accordance with the Guide for the Care and Use of Laboratory Animals. Ten (10) animals were used for Evan's Blue (EB) absorbance analysis. Eighteen (18) animals were used for immunohistochemisty (IHC) staining and western blot analysis. Nine (9) animals were used for microvessel isolation and subsequent western blot analysis (Fig. 1). Bryostatin-1 was administered by intraperitoneal injection $(2.5 \mathrm{mg} / \mathrm{kg}$ ) (Tan et al 2013), 5 minutes after blast exposure for all blast + bryostatin-1 groups. Bryostatin-1 was dissolved in vehicle (10\% ethanol in $0.9 \%$ normal saline). Bryostatin-1 was obtained by Dr. Daniel Alkon from the National Cancer Institute, National Institutes of Health, Bethesda, MD. The dose of bryostatin-1 was selected in accordance with animal protocols and according to a dose response curve showing that bryostatin- 1 enters the brain but is maintained well below the maximal tolerated dose (Berkow et al 1993, Sun \& Alkon 2006). Control animals were anesthetized and injected with vehicle. Bryostatin-1 is a potent modulator of PKC isozymes with selectivity for PKC $\alpha$ and $\varepsilon$ (Sun et al 2014).

\section{$\underline{\text { Blast exposure }}$}

The animal was oriented perpendicular to the blast apparatus, with the driver section placed near the right side of the skull. A polyvinyl chloride shield protected the 
rest of the body. We utilized a membrane with 0.005 inch thickness to generate a blast exposure with peak reflected overpressure of $~ 50$ PSI, and peak incident overpressure of $\sim 15$ PSI (Figure 4.1 B-C). The exposure produced moderate blast injury as previously characterized (Turner et al 2013). The primary extent of injury has been localized to the left hemisphere due to coup and counter-coup acceleration/deceleration mechanisms (Turner et al 2012b). Briefly, nitrogen gas builds pressure behind a clear polyester film that subsequently ruptures to produce a blast wave. Piezoelectric sensors (Model 102AO5; PCB Piezotronics) were placed in the reflected and incident positions at the exit of the shock tube and data was recorded with a data acquisition board (DAQ 23GF, National Instruments) and sensor signal conditioner (482C Series; PCB Piezotronics). LabView software version 12.0 (National Instruments) was used to reconstruct accurate pressure recording graphs (Figure 4.1 D). The short driver section generates briefduration waves comparable to those seen from improvised explosive devices when scaled properly for the rodents size based on principles previously highlighted by Bass and colleagues (Bass et al 2012, Panzer et al 2014b).

\section{Evan's blue}

Groups for Evan's Blue (EB) included: control $(n=3)$, single blast $(n=4)$, and single blast + bryostatin-1 $(n=3)$. BBB permeability was assessed 6 hours after blast exposure using EB as a tracer molecule. EB binds to albumin, and albumin will only enter the brain if the $\mathrm{BBB}$ is compromised. Animals were anesthetized with inhaled 4\% isoflurane (Halocarbon) and maintained with $2 \%$ isoflurane before normal saline containing $\mathrm{EB}(2 \%, 5 \mathrm{ml} / \mathrm{kg})(2 \% \mathrm{w} / \mathrm{v}$ in saline) was administered intravenously (femoral vein) 30 minutes prior to perfusion. The rats were transaortally perfused with normal 
saline for 10 minutes. The brains were excised; meninges and ependymal organs removed, hemispheres separated, and left prefrontal cortex sectioned. The prefrontal cortex is one of the brain regions most susceptible to BBB disruption following injury (Yen et al 2013). The left prefrontal cortex samples were then weighed, and homogenized in $1 \mathrm{ml}$ of $50 \%$ trichloroacetic acid (TCA). The resulting suspensions were placed in $0.5 \mathrm{~mL}$ aliquots. The aliquots were incubated for 24 hours at $37^{\circ}$, and centrifuged at $10,000 \times \mathrm{g}$ for 10 minutes. The supernatant was collected and measured by absorbance spectroscopy at $620 \mathrm{~nm}$ for EB determination. Calculations were based on external standard readings $(50 \mu \mathrm{g} / \mathrm{mL}$ of EB dissolved in saline with 8 serial dilutions). The extravasated dye in brain tissue was expressed as ng EB/mg of brain tissue.

\section{Microvessel isolation}

Rats were anesthetized with $4 \%$ isoflurane, decapitated, and the brain was removed. The brain was placed in $10 \mathrm{~mL}$ of MVI Buffer two $(6.02 \mathrm{~g} / \mathrm{L} \mathrm{NaCl}, 0.35 \mathrm{~g} / \mathrm{L}$ $\mathrm{KCl}, 0.37 \mathrm{~g} / \mathrm{L} \mathrm{CaCl}$, $0.16 \mathrm{~g} / \mathrm{L} \mathrm{KH} 2 \mathrm{PO} 4,0.3 \mathrm{~g} / \mathrm{L} \mathrm{MgSO}$, and $3.57 \mathrm{~g} / \mathrm{L}$ HEPES) with protease inhibitor cocktail. The meninges and choroid plexus were removed and the cerebral hemispheres were placed in a glass homogenization tube with $4 \mathrm{~mL}$ of MVI Buffer one (6.02 g/L NaCl, 0.35 g/L KCl, 0.37 g/L CaCl2, 0.16 g/L KH2PO4, 0.3 g/L MgSO4, 3.57 g/L HEPES, 2.1 g/L NaHCO3, 1.80 g/L Glucose, 0.11 g/L Na Pyruvate, and $10 \mathrm{~g} / \mathrm{L}$ Dextran $(64 \mathrm{~K}))$ and protease inhibitor cocktail. MVI one and two were $\mathrm{pH}$ adjusted to 7.4. With a Teflon pestle, the tissue was homogenized and filtrate placed in a $16 \mathrm{~mL}$ tube with $4 \mathrm{~mL}$ of $26 \%$ dextran. The tubes were centrifuged at 5,800 $\mathrm{g}$ g for 10 min at $4{ }^{\circ} \mathrm{C}$. The supernatant was aspirated and the pellet was re-suspended in $5 \mathrm{~mL}$ of MVI buffer two with protease inhibitor cocktail. The homogenate was filtered through 
$100 \mu \mathrm{m}$ filter, and centrifuged at $5,800 \mathrm{xg}$ for $5 \mathrm{~min}$ at $4^{\circ} \mathrm{C}$. The supernatant was decanted and microvessel pellet saved for western blot analysis.

\section{Western blot}

Western blot analysis was performed similar to work previously published by our laboratory (Dinapoli et al 2010). Briefly, 24 hours after blast exposure, animals were anesthetized with $4 \%$ isoflurane, brains were removed by decapitation, and the brains were subsequently sectioned. Groups included: control, single blast, and single blast + bryostatin-1. Protein samples from the left prefrontal cortex were dissolved in $0.5 \mathrm{~mL}$ hot (85-95o C) 1\% sodium docecyl sulfate (SDS), sonicated, and a Bicinchoninic Acid (BCA) protein assay kit (Thermo Fisher Scientific, Rockfield, IL) was used to determine protein concentration. The pre-stained standard SeeBlue ${ }^{\circledR}$ Plus2 (Life Technologies, Carlsbad, CA) was used. 2X Lammeli buffer combined with $30 \mu \mathrm{g}$ of protein was loaded per well and run on a Bolt $®$ Mini tank system (Life Technologies) with pre-cast Bolt $₫$ Bis-Tris Plus 10\% 12-well gels (Life Technologies). PVDF membranes (Bio-Rad, Contra Costa, CA) were soaked in methanol and used for wet transfer (Bio-Rad) at 60 volts for 2.5 hours. Primary antibodies used were occludin rabbit 1:1000 (Life Technologies), PKC $\alpha, \delta, \varepsilon$ mouse 1:200 (Santa Cruz Biotechnology, Santa Cruz, CA), and VE-Cadherin rabbit 1:1000 (Cell Signaling, Danvers, MA). Microvessel isolation tissue was used to detect changes in PKC isozymes. LI-COR secondary antibodies IRDye ${ }^{\circledR} 800 \mathrm{CW}$ and IRDye® 680RD (LI-COR, Lincoln, NE) were used with an Odyssey fluorescent scanner at wavelengths 800 or 700 , intensity 6.0 , and 84 resolution with high image quality. Images were analyzed after background subtraction, and normalized to $\beta$-actin to give relative overall intensity. 


\section{Immunohistochemistry}

Experimental groups included control $(\mathrm{n}=3)$, single blast $(\mathrm{n}=3)$, and single blast + bryostatin-1 ( $n=3)$. Rats were anesthetized with $4 \%$ isoflurane and transcardially perfused with $0.9 \%$ saline followed by $4 \%$ paraformaldehyde at 24 hours after blast exposure. The rats were decapitated, and brains were removed and stored in $4 \%$ paraformaldehyde until processing. The tissue was sectioned and paraffin embedded. $6 \mu \mathrm{m}$ slices from the left prefrontal cortex were cut with a Leica RM2235 microtome (Leica Microsystems, Wetzlar, Germany) and mounted on slides. Slides were washed in xylene, $100 \% \mathrm{EtOH}$ and $95 \% \mathrm{EtOH}$ for 5 minutes each in order to remove the paraffin, followed by rehydration in $\mathrm{dH} 2 \mathrm{O}$ for 5 minutes. Using 10\% methanol and 10\% $\mathrm{H} 2 \mathrm{O} 2$ in Dulbecco's phosphate buffered saline (DPBS), slides were quenched for 15 minutes, followed by three rinses in DPBS for 5 minutes each. The slides were then added to permeabilizing solution (1.8\% L-Lysine, $4 \%$ horse serum, and $0.2 \%$ Triton X-100 in DPBS) for 30 minutes. Slices were circumscribed and incubated overnight with a primary antibody and $4 \%$ horse serum. The next day slides were rinsed and incubated for 3-hours with a fluorescent secondary antibody. A second set of primary and secondary antibodies were used in the case of co-localization staining. Slides were then fixed with Permount mounting media. Primary antibodies include: PKC $\alpha, \delta$, and $\varepsilon$ mouse 1:500 (Santa Cruz Biotechnology), Claudin-5 mouse 1:200 (Life Technologies), occludin rabbit 1:200 (Life Technologies), VE-Cadherin rabbit 1:150 (Cell Signaling), ZO-1 Alexa Fluor® 488 mouse 1:200 (Life Technologies), Glial Fibrillary Acidic Protein (GFAP) mouse 1:150 (Cell Signaling), GFAP rabbit 1:500 (DAKO, Glostrup, Denmark), Neuronal/glia proteoglycan 2 (NG-2) rabbit 1:500 (Santa Cruz Biotechnology) and Von Willebrand 
Factor (VWF) rabbit 1:200 (Sigma Aldrich, St. Louis, MO). Secondary antibodies include Alexa Fluor 594 goat anti-mouse 1:1000 (Life Technologies), Alexa Fluor 594 goat anti-rabbit 1:500 (Life Technologies), Alexa Fluor 488 donkey anti-mouse 1:500 (Life Technologies), and Alexa 488 donkey anti-rabbit 1:1000 (Life Technologies). 20 cells per slide were randomly selected, outlined, and measured with ImageJ software $(\mathrm{NIH})$ by an observer blinded to experimental group. Density was adjusted per mean area to give corrected total cell fluorescence normalized to background for claudin-5, occludin, VE-Cadherin, ZO-1, and PKC $\alpha, \delta$, and $\varepsilon$. Co-localization was quantified with the ImageJ plugin Just Another Co-localization plugin to give Pearson's coefficient for controls and overlap coefficient for treatment groups (Bolte \& Cordelieres 2006). For percentage of co-localization, an observer blinded to experimental group counted 100 cells and the ratio of positive cells to total cells was recorded. $\chi^{2}$ analyses was used to compare between groups.

\section{$\underline{\text { Statistical Analysis }}$}

Biochemical assay data were analyzed using GraphPad Prism 6.0 (GraphPad Software, Inc., La Jolla, CA) by an observer blinded to experimental group. A one-way ANOVA with Tukey's post-hoc was used to compare across groups for EB absorbance, western blot analysis, and IHC quantification. Overlap coefficient was calculated via the ImageJ plugin with the following equation: $\mathrm{k}^{\wedge} 2=\mathrm{k} 1 * \mathrm{k} 2$ with all values adjusted to threshold. Pearson's coefficient was obtained for controls. $\chi 2$ analyses was used to compare the percentages of positive co-localized cells between groups. $\mathrm{P}<0.05$ was considered statistically significant for all data analyzed. 


\section{RESULTS}

\section{Bryostatin-1 significantly modulates PKC $\alpha$ and $\varepsilon$ but not PKC $\delta$ expression after blast}

Bryostatin-1 has previously been shown to be neuroprotective following mild TBI in a murine model by reducing $\beta$-secretase and $\beta$ amyloid production (Zohar et al 2011). Furthermore, our lab has published that bryostatin-1 decreases PKC $\alpha$ and increases PKC $\varepsilon$ following neural injury in an aged-female Sprague-Dawley model (Tan et al 2013). The role bryostatin-1 plays in regulating PKC activity at the neurovascular unit has yet to be determined. We examined perivascular regions of the left prefrontal cortex using quantitative IHC. A significant difference in PKCa was observed between groups $(F(2,24)=7.743, p<0.01) 24$ hours after injury. Post-hoc comparison revealed a significant difference between control and single blast groups $(t=5.043, p<0.01)$ and between single blast and single blast + bryostatin-1 groups $(\mathrm{t}=4.559, \mathrm{p}<0.01)$ (Figure 4.2 A-G). A significant difference in PKC $\delta$ was observed between groups $(\mathrm{F}(2,27)=16.73$, p $<0.001) 24$ hours after injury. Post-hoc comparison revealed a significant difference between control and single blast groups $(\mathrm{t}=8.049, \mathrm{p}<0.001)$ and between control and single blast + bryostatin-1 groups $(\mathrm{t}=5.292, \mathrm{p}<0.01)($ Figure $4.2 \mathrm{H}-\mathrm{N})$. No significant difference was seen between single blast and single blast + bryostatin- 1 , consistent with the notion that bryostatin-1 negligibly alters PKC $\delta$ expression. A significant difference in PKC $\varepsilon$ was observed between groups $(F(2,27)=14.73, \mathrm{p}<0.001) 24$ hours after injury. Post-hoc comparison revealed a significant difference between control and single blast + bryostatin- 1 groups $(\mathrm{t}=7.509, \mathrm{p}<0.001)$ and between single blast and single blast + bryostatin-1 groups $(\mathrm{t}=5.133, \mathrm{p}<0.01)$ (Figure $4.2 \mathrm{O}-\mathrm{U})$. We also examined changes in 
PKC expression using western blot analysis. A significant difference between groups was observed for $\mathrm{PKC} \alpha(\mathrm{F}(2,14)=7.672, \mathrm{p}<0.01)$. Post-hoc comparison revealed a significant difference between control and single blast $(\mathrm{t}=4.576, \mathrm{p}<0.05)$, and between single blast and single blast + bryostatin-1 $(\mathrm{t}=5.113, \mathrm{p}<0.01)$ (Figure $4.3 \mathrm{~A})$. A significant difference between groups was observed for $\operatorname{PKC}(F(2,6)=13.25, p<0.01)$. Post-hoc comparison revealed a significant difference between control and single blast $(\mathrm{t}=7.197$, $\mathrm{p}<0.01)$, and between control and single blast + bryostatin- $1(\mathrm{t}=4.549, \mathrm{p}<0.05)$ (Figure 4.3 B). A significant difference between groups was observed for $\operatorname{PKC} \varepsilon(\mathrm{F}(2,15)=10.05$, $\mathrm{p}<0.01)$. Post-hoc comparison revealed a significant difference between control and single blast $(\mathrm{t}=4.546, \mathrm{p}<0.05)$, and between control and single blast + bryostatin- 1 $(\mathrm{t}=6.102, \mathrm{p}<0.01)($ Figure $4.3 \mathrm{C})$.

\section{Bryostatin-1 maintained BBB integrity following blast exposure}

BBB integrity can be measured by EB extravasation into the brain parenchyma (Abdul-Muneer et al 2013). EB binds to albumin, and albumin does not readily penetrate an intact BBB. NG-2 is a pericyte proteoglycan that is increased during BBB disruption and subsequent neovascularization (Girolamo et al 2013). Disruption of the BBB has multiple effects including neuroinflammation and formation of reactive oxygen species (Abdul-Muneer et al 2014). A significant difference between groups was observed for EB extravasation 6 hours after blast exposure $(F(2,7)=7.38, p<0.05)$ for the left prefrontal cortex (Figure 4.4 A). Post-hoc comparison was significant between control and single blast $(\mathrm{t}=4.808, \mathrm{p}<0.05)$ and between single blast and single blast + bryostatin- $1(\mathrm{t}=4.347$, $\mathrm{p}<0.05)$. No significant difference was seen between control and single blast + bryostatin-1. Bryostatin-1 successfully preserved the integrity of the BBB apparent on 
gross exam of the left hemisphere (Figure 4.4 B). A significant difference in NG-2 was observed 24 hours after blast exposure $(\mathrm{F}(2,27)=20.42, \mathrm{p}<0.001)$ for the left prefrontal cortex (Figure 4.4 C-H). Post-hoc comparison was significant between control and single blast $(\mathrm{t}=5.504, \mathrm{p}<0.01)$, and between single blast and single blast + bryostatin- $1(\mathrm{t}=8.961$, $\mathrm{p}<0.001$ ), but not between control and single blast + bryostatin- 1 .

\section{PKC modulation alters tight junction proteins at the $B B B$}

Activation of PKC isozymes is thought to play a crucial role in BBB disruption (Shao \& Bayraktutan 2014). PKCa, in particular, has been linked to redistribution of tight junction proteins leading to increased vascular permeability (Stamatovic et al 2006). $\mathrm{PKC} \alpha$ is also known to activate sonic hedgehog protein leading to dysfunctional synthesis of tight junction proteins (Guo et al 2012, Xia et al 2013). Alternatively, increasing $\mathrm{PKC} \varepsilon$ facilitates tight junction protein translocation from the nucleus to the plasma membrane (Chamorro et al 2009). Gross examination of the neurovascular unit (VWF co-localized with GFAP) revealed histological disruption of the BBB following blast injury but maintenance of barrier integrity when bryostatin-1 was administered following blast exposure (Figure 4.5 A-C). A significant difference between groups was not observed for Claudin-5 (Figure 4.5 D-J) using fluorescent IHC. A significant difference between groups was observed for VE-Cadherin $(F(2,6)=18.58, p<0.01)$ using western blot. Post hoc comparison revealed a significant difference between control and single blast $(\mathrm{t}=4.571, \mathrm{p}<0.05)$, and between control and single blast + bryostatin- 1 $(t=8.616, p<0.01)$ (Figure 4.6 A). A significant difference between groups was observed for occludin $(\mathrm{F}(2,6)=49.02, \mathrm{p}<0.001)$ using western blot. Post hoc comparison revealed a significant difference between control and single blast $(\mathrm{t}=6.955, \mathrm{p}<0.01)$, between 
control and single blast + bryostatin- $1(\mathrm{t}=14, \mathrm{p}<0.001)$, and between single blast and single blast + bryostatin-1 $(\mathrm{t}=7.048, \mathrm{p}<0.01)($ Figure 4.6 B). A significant difference between groups was observed for ZO-1 $(\mathrm{F}(2,26)=35.68, \mathrm{p}<0.001)$ using fluorescent IHC. Post hoc comparison revealed a significant difference between control and single blast + bryostatin-1 $(\mathrm{t}=11.45, \mathrm{p}<0.001)$, and between single blast and single blast + bryostatin-1 ( $\mathrm{t}=8.553, \mathrm{p}<0.001)$ (Figure 4.6 C-I). Additionally, a significant difference between groups was observed for VE-Cadherin $(\mathrm{F}(2,27)=5.9, \mathrm{p}<0.05)$ using fluorescent IHC. Post hoc comparison revealed a significant difference between control and single blast $(\mathrm{t}=4.016, \mathrm{p}<0.05)$, and between control and single blast + bryostatin- $1(\mathrm{t}=4.375$, $\mathrm{p}<0.05$ ) (Figure 4.7 A-G). A significant difference was also observed between groups for occludin $(F(2,27)=79.88, \mathrm{p}<0.001)$. Post hoc comparison revealed a significant difference between control and single blast $(\mathrm{t}=8.304, \mathrm{p}<0.001)$, between control and single blast + bryostatin-1 $(\mathrm{t}=17.86, \mathrm{p}<0.0001)$, and between single blast and single blast + bryostatin-1 $(\mathrm{t}=9.557, \mathrm{p}<0.001)($ Figure $4.7 \mathrm{H}-\mathrm{N})$.

\section{$\underline{P K C} \alpha, \delta$, and $\varepsilon$ are co-localized with endothelial cells but not astrocytes after blast} exposure

The neurovascular unit and the associated BBB consists of endothelial cells, astrocytes, and pericytes that form a structural basement membrane (Amtul \& Hepburn 2014). The unit acts as a consortium that dictates vascular constriction and dilation in response to stimuli (Muoio et al 2014). The role of PKC isozymes in mediating changes to vasculature tone following TBI has yet to be elucidated. We examined the extent of colocalization for PKC $\alpha, \delta$, and $\varepsilon$ with endothelial cells (VWF) or astrocytes (GFAP) in the left prefrontal cortex of control animals and single blast exposed animals 24 hours after 
injury. The Pearson's coefficient for control animals for PKC $\alpha$ and VWF was $r=0.395$ (Figure 4.8 A-F). The overlap coefficient for PKC $\alpha$ and VWF after single blast exposure (threshold $\mathrm{A}=20$, threshold $\mathrm{B}=14$ ) was $\mathrm{r}=0.954$ with $\mathrm{k} 1=0.734$ and $\mathrm{k} 2=1.238$ (Figure 4.8 G-L). The Pearson's coefficient for control animals for PKC $\alpha$ and GFAP was $r=0.351$ (Figure 4.8 M-R). The overlap coefficient for PKC $\alpha$ and GFAP after single blast exposure (threshold $\mathrm{A}=29$, threshold $\mathrm{B}=22$ ) was $\mathrm{r}=0.379$ with $\mathrm{k} 1=0.768$ and $\mathrm{k} 2=0.187$ (Figure 4.8 S-X). The Pearson's coefficient for control animals for PKC $\delta$ and VWF was $r$ $=0.61$ (Figure 4.9 A-F). The overlap coefficient for PKC $\delta$ and VWF after single blast exposure (threshold $\mathrm{A}=34$, threshold $\mathrm{B}=14$ ) was $\mathrm{r}=0.88$ with $\mathrm{k} 1=0.444$ and $\mathrm{k} 2=1.744$ (Figure 4.9 G-L). The Pearson's coefficient for control animals for PKCס and GFAP was $\mathrm{r}=0.029$ (Figure 4.9 M-R). The overlap coefficient for PKC $\delta$ and GFAP after single blast exposure (threshold $\mathrm{A}=52$, threshold $\mathrm{B}=20$ ) was $\mathrm{r}=0.449$ with $\mathrm{k} 1=0.227$ and k2=0.89 (Figure 4.9 S-X). The Pearson's coefficient for control animals for PKC $\varepsilon$ and VWF was $r=0.004$ (Figure 4.10 A-F). The overlap coefficient for PKCE and VWF after single blast exposure (threshold $\mathrm{A}=44$, threshold $\mathrm{B}=23$ ) was $\mathrm{r}=0.978$ with $\mathrm{k} 1=0.765$ and k2=1.249 (Figure 4.10 G-L). The Pearson's coefficient for control animals for PKC $\varepsilon$ and GFAP was $r=0.133$ (Figure 4.10 M-R). The overlap coefficient for PKC $\varepsilon$ and GFAP after single blast exposure (threshold $\mathrm{A}=24$, threshold $\mathrm{B}=22$ ) was $\mathrm{r}=0.394$ with $\mathrm{k} 1=0.286$ and $\mathrm{k} 2=0.543$ (Figure $4.10 \mathrm{~S}-\mathrm{X}$ ). We examined the percentage of positive cells for PKC $\alpha$ co-localized with VWF in perivascular regions of the left prefrontal cortex 24 hours after injury (Supplementary Figure 1). The ratio of positively stained cells to total cells for control was 18/100, for blast-exposed animals 57/100, and for blast exposed 
animals treated with bryostatin-1 36/100. $\chi 2=20.595$ with two degrees of freedom and a two-tailed $\mathrm{P}$ value equal to 0.0001 .

\section{DISCUSSION}

Bryostatin-1 has been successfully used in numerous animal models of neural injury including TBI, ischemic stroke, auditory neurodegeneration, and Alzheimer's disease, and is currently under investigation in clinical trials (Lallemend et al 2005, Sun \& Alkon 2014, Sun et al 2009, Zohar et al 2011). Recent findings have shown that secondary injury mechanisms such as neuroinflammation, endoplasmic reticulum stress, and PKC activity are conserved despite the type of injury (Hoozemans \& Scheper 2012, Lucke-Wold et al 2014d, Wu et al 2014). The timing of activity might vary but the pathways are similar and theoretically targeted in a similar fashion. For example, bryostatin-1 protects against loss of pre-synaptic synaptophysin and loss of post-synaptic spinophylin following TBI in mice (Zohar et al 2011). In an aged-female SpragueDawley stroke model, bryostatin-1 improved survival and reduced ischemic damage (Tan et al 2013). Likewise, bryostatin-1 decreased apoptosis and prevented deficits in synaptogenesis in a global model of cerebral ischemia (Sun et al 2009, Sun et al 2008). Despite the different injury models bryostatin-1 was a successful therapeutic because it upregulates PKC activity early and downregulates activity when used at excess doses or at chronic time points (Sun \& Alkon 2013). At the dose used in this study, it is possible that an early activation of PKC $\alpha$ is followed by a drastic downregulation observed at 24 hours. PKC $\varepsilon$ may also be increased not only due to upregulation but also from increased synthesis (Hongpaisan et al 2011). Future studies will be required to look at PKC changes at both earlier time points and also the beneficial effects of bryostatin- 1 when 
administered at subacute time points. In Alzheimer's disease early upregulation of PKC activity enhances neuronal repair and decreases amyloid mediated degeneration while maintaining long-term potentiation (Kim et al 2012a, Nelson \& Alkon 2009). In addition, bryostatin-1 facilitates neurite outgrowth, maintains cognitive function (Lallemend et al 2005), and enhances synaptic connectivity and spatial learning (Sun et al 2014). For affective disorders bryostatin-1 downregulates ERK dependent PKC activity at late time points, which can have beneficial effects for treating substance abuse, depression, and aversive emotional memories (Mogha et al 2012, Sun \& Alkon 2014). Similar molecular PKC targets appear to be altered in TBI (Luo et al 2014b).The potential for bryostatin-1 as a treatment for TBI warrants further investigation. The necessity for different dosing strategies as well as altering the timing of dosing must be carefully considered in a series of future studies. Furthermore, specific activation of select isozymes will offer compelling insight into their protective properties following TBI. In this study, we examined how a single $2.5 \mathrm{mg} / \mathrm{kg}$ dose of bryostatin- 1 affects BBB integrity acutely following blast-induced TBI.

We found that a single moderate TBI significantly increased EB extravasation into the left prefrontal cortex six hours after injury. Bryostatin-1 administration ameliorated this disruption. A potential mechanism by which this occurs is through regulation of $\mathrm{PKC} \alpha$ and $\mathrm{PKC} \varepsilon$, which subsequently increases the tight junction proteins VE-Cadherin, occludin, and ZO-1 at the BBB. It has been previously shown in an ischemic stroke model that inhibiting PKCa increases the tight junction proteins ZO-1, occludin, and VE-Cadherin ( $\mathrm{Yu}$ et al 2012). PKCa has limited regulatory function for claudin-5 accounting for the non-significant findings observed. Tight junction proteins 
overall are essential for maintaining limited and selective permeability into the brain parenchyma and preventing neuroinflammation (Chodobski et al 2011). A switch to PKC $\alpha$ activation following TBI has been shown to accelerate neuroinflammation (Dai et al 2010). Our data show that bryostatin-1 decreased levels of PKC $\alpha$ when given after blast. A separate isozyme, $\mathrm{PKC} \delta$, mediates vasoconstriction, but its role in tight junction disruption is poorly understood (Novokhatska et al 2013). Our data show that bryostatin-

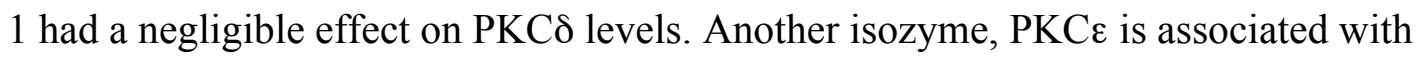
neuroprotection in various models of brain injury (Feng et al 2013). Bryostatin-1 was previously shown to regulate tight junction proteins in vitro via modulation of PKC $\varepsilon$ (Yoo et al 2003). PKCE is essential for exportation of zona occluden proteins to the site of functional activity (Chamorro et al 2009). We found that bryostatin-1 significantly elevated PKC $\varepsilon$ when administered after blast injury.

Increasing VE-Cadherin, occludin, and ZO-1 strengthens the BBB therefore maintaining its integrity (Liu et al 2014a). Other therapeutic approaches for TBI such as hyperbaric oxygen and sevoflurane were previously shown to significantly increase tight junction protein expression (Chen et al 2014, Thal et al 2012). Our data provide compelling evidence that bryostatin-1 also increases tight junction proteins leading to maintenance of the BBB and decreased permeability after blast injury. Future work will examine the functional capacity of these tight junction proteins as well as their efficient means of trafficking (McCaffrey \& Davis 2012). Utilizing in vitro modeling with astrocyte and endothelial co-culture may represent an effective way to examine functional capacity (Yang et al 2012). Designing an effective in vitro model of blast TBI and BBB dynamics is an area requiring focused investigation (Arun et al 2013b, Effgen 
et al 2014). Another avenue requiring further investigation is how changes in PKC function affect the gliovascular unit. Our data show that PKC changes were localized to the endothelial cells not glia. What has yet to be discovered is how cytokines released from endothelial cells may alter the surrounding gliovascular unit (Alves 2014). In order to investigate this important area, specific PKC modulators such as (Alphatomega; HFKKQGSFAKKK-NH(2)) for PKC $\alpha$ and the PKC $\varepsilon$ peptide activator must be used in future investigative studies (Kang et al 2008, Teng et al 2008). A malfunctioning gliovascular unit can contribute to chronic neurodegeneration (Barzilai 2013).

An area of ongoing research is how the progression of acute BBB disruption leads to chronic detrimental outcomes. Subtle changes can occur in a young developing brain post TBI that often take years to manifest into symptoms (Adelson et al 2013). Microbleeds from a disrupted BBB following TBI can cause long-term negative outcomes such as glial scarring and white matter degeneration (Glushakova et al 2014, Lucke-Wold et al 2014c, Wu et al 2012). These markers of injury have been associated with mid-to-late life diseases such as chronic traumatic encephalopathy, autoimmune encephalomyelitis, vascular dementia, and early onset Alzheimer's disease (Marcelo \& Bix 2014, Stein et al 2014, Turner et al 2012a). Interestingly, stabilization of the BBB through PKC modulation decreases chronic negative effects acutely (Lanz et al 2013). If BBB disruption does occur, calcium imbalance triggers a host of downstream events. Current understanding indicates that glia communicate with neurons through calcium signaling, and calcium signaling is often disrupted following TBI (De Bock et al 2014). Disrupted calcium signaling can lead to activation of endoplasmic reticulum stress and mitochondrial dysfunction (Naviaux 2014). Long term outcomes include the formation of 
reactive oxygen species, amyloid pathology, and tau hyperphosphorylation (Eckert et al 2013). PKC isozymes are known tau kinases that are increased in neurodegenerative disease (Kamat et al 2014). Long-term bryostatin-1 treatment has been shown to modulate tau hyperphosphorylation in a murine model of dementia (Ekinci \& Shea 1997, Sun \& Alkon 2014). A large prospective cohort study has recently shown that veterans diagnosed with TBI have a 1.57 adjusted hazard ratio for the onset of dementia compared to controls (Barnes et al 2014). Ongoing work will investigate the long-term role bryostatin-1 treatment plays in preventing neurodegeneration following repetitive blast exposure. Appropriate dosing strategies will be carefully developed for single and repetitive injury. The effects of bryostatin-1 are likely two fold: protection of BBB acutely and preservation of neuronal stability chronically. Timing and appropriate dosing is a necessity to tease out the most appropriate protective treatment windows. PKC modulation offers a promising therapeutic approach for preventing the chronic sequelae associated with neurotrauma.

\section{ACKNOWLEDGEMENTS}

The authors would like to thank Ryan W. Holt for his help with IHC and western blot analysis. We thank Diana Richardson CDC/NIOSH for tissue preparation for IHC. The authors also thank Dr. Rae Matsumoto and Dr. Steven Frisch for use of western blot resources in their respective laboratories. We thank James E. Robson and Peter Bennett

for construction of the blast model and Dr. Robert Gettens and Nic St. John for design of the blast model. A Research Funding and Development Grant (RFDG) from the West Virginia University Health Sciences Center Office of Research and Graduate Education 
supported this work along with WVU Department of Neurosurgery. R.C.T. was supported by a NIH training grant (GM08174). 


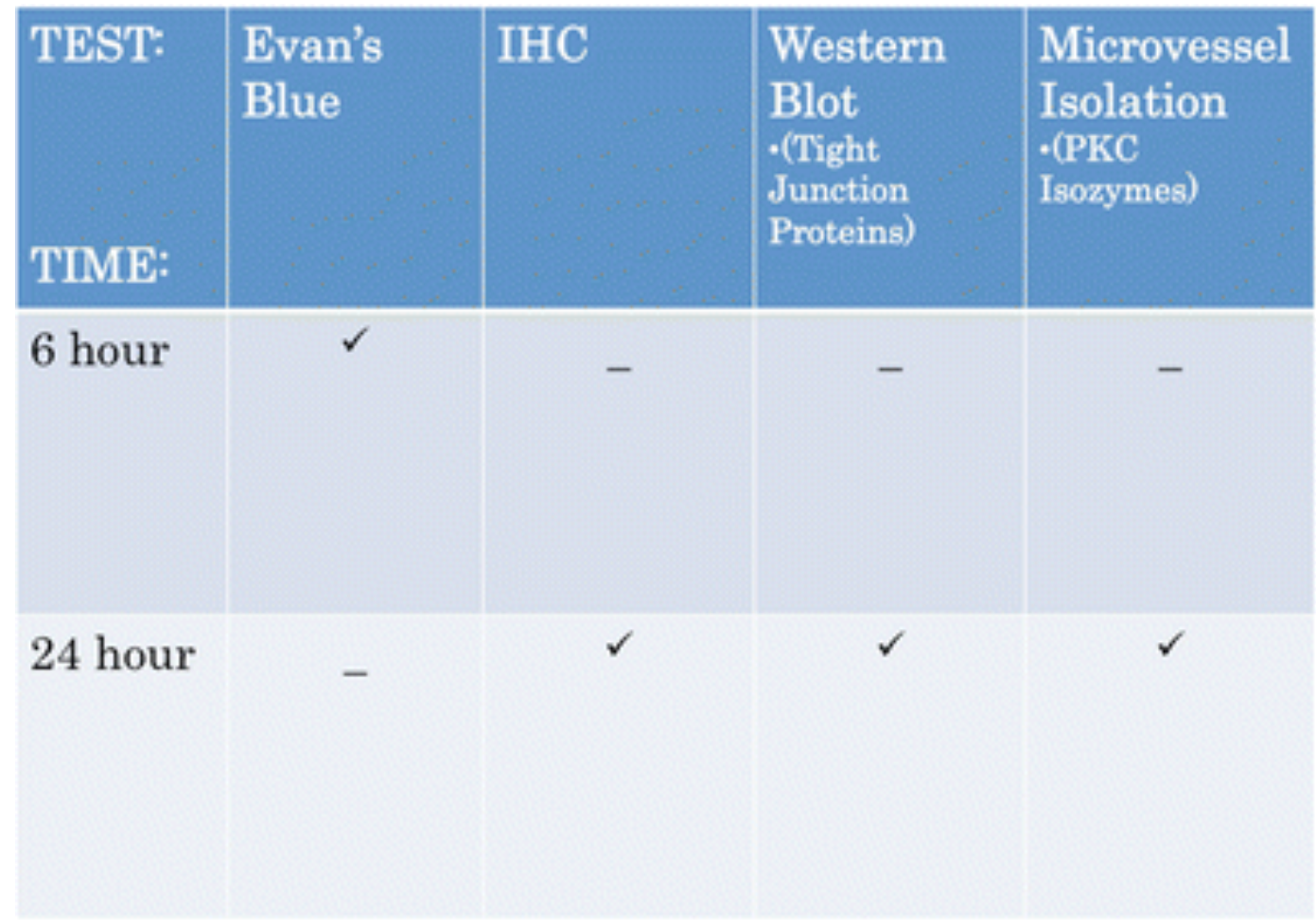

Figure 4.1: Table showing the experimental techniques used for each time point. 
CTRL

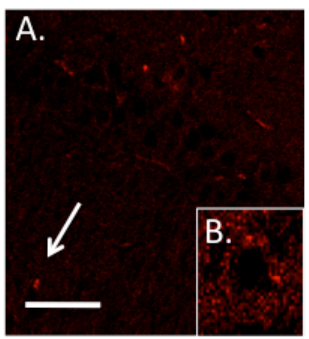

H.
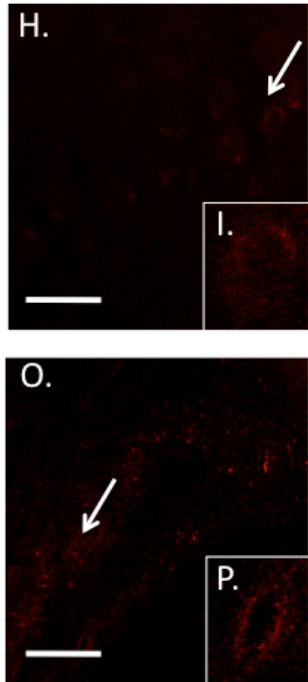

SB
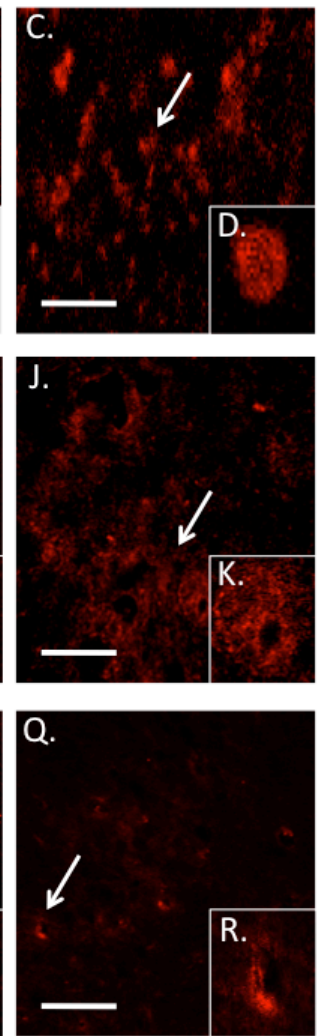

$\mathrm{SB}+$ Bryo
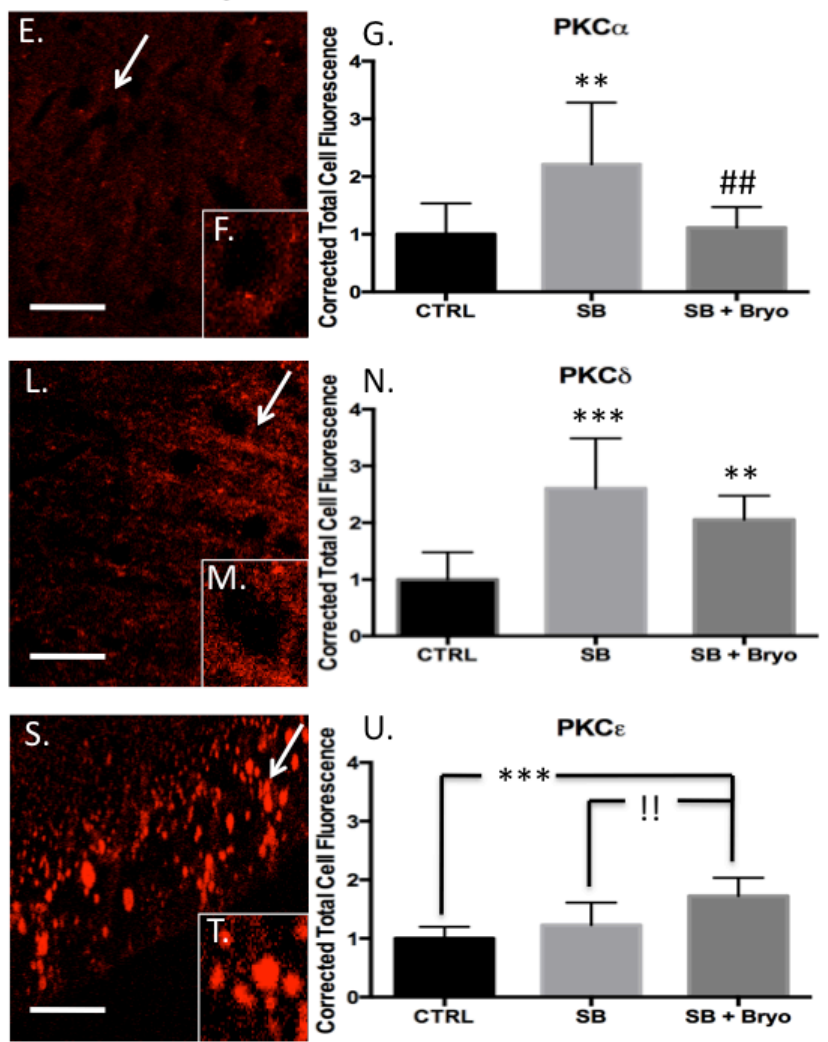

Figure 4.2: Bryostatin-1 decreases PKC $\alpha$ and increases PKCE 24 hours after blast exposure. Our data show that bryostatin-1 has a profound effect after blast traumatic brain injury using fluorescent IHC. Scale bar $=100 \mu \mathrm{m}$ in left prefrontal cortex. PKC $\alpha$ control (A) with inlay (B) compared to single blast exposure (C) with inlay (D), and single blast exposure + bryostatin-1 (E) with inlay (F) showed significant difference between groups. Post hoc comparison between control and single blast $(* * p<0.01)$ and between single blast and single blast + bryostatin-1 (\#\# $\mathrm{p}<0.01)$ as depicted in bar graph (G). PKC $\delta$ control (H) with inlay (I) compared to single blast exposure (J) with inlay (K), and single blast exposure + bryostatin-1 (L) with inlay (M) showed significant difference between groups. Post hoc comparison between control and single blast $(* * *$ $\mathrm{p}<0.001)$ and between control and single blast + bryostatin- $1(* * \mathrm{p}<0.01)$ as depicted in bar graph $(\mathbf{N})$. PKC $\varepsilon$ control $(\mathbf{O})$ with inlay $(\mathbf{P})$ compared to single blast exposure $(\mathbf{Q})$ 
with inlay $(\mathbf{R})$, and single blast exposure + bryostatin-1 (S) with inlay $(\mathbf{T})$ showed a significant difference between groups. Post hoc comparison between control and single blast + bryostatin-1 $(* * * p<0.001)$ and between single blast and single blast + bryostatin$1(! ! \mathrm{p}<0.01)$ as depicted in bar graph $(\mathbf{U})$. 
A.

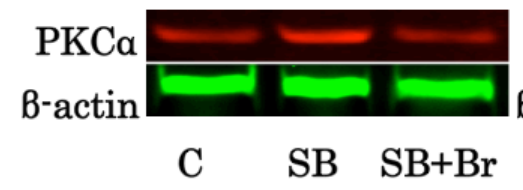

PKC $\alpha$

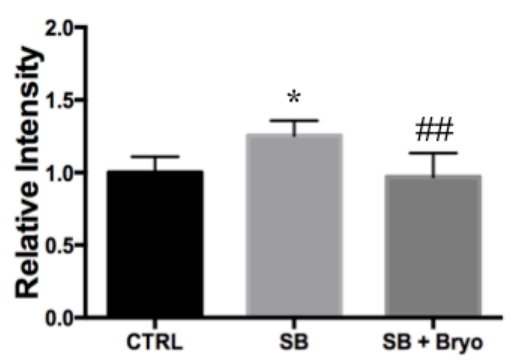

B. PKC8

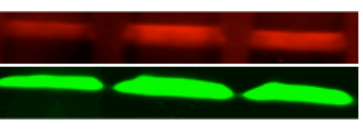

C $\quad$ SB $\quad$ SB+Br

PKC $\delta$

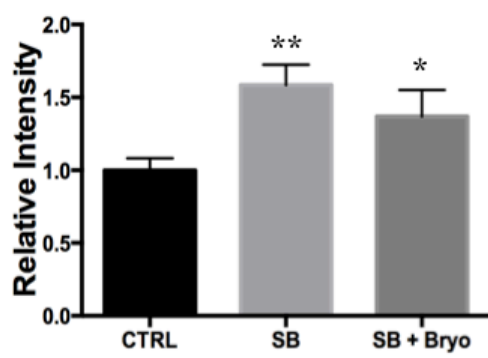

C.

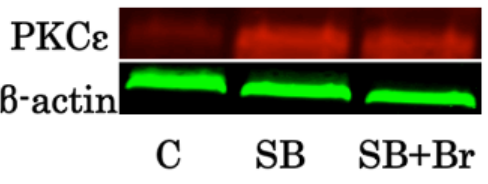

Figure 4.3: Bryostatin-1 is a potent PKC modulator. It has been used to regulate PKC activity in a time-specific manner for multiple neural injury models. Protein concentrations were measured in the left prefrontal cortex 24 hours after blast expsoure using western blot analysis. A significant difference between groups was observed for PKC $\alpha$. Post-hoc comparison between control and single blast $(* \mathrm{p}<0.05)$, and between single blast and single blast + bryostatin-1 (\#\# $<<0.01)(\mathbf{A})$. A significant difference between groups was observed for PKC $\mathrm{P}$. Post-hoc comparison between control and single blast (** $\mathrm{p}<0.01)$, and control and single blast + bryostatin-1 $(* \mathrm{p}<0.05)(\mathbf{B})$. A significant difference between groups was observed for PKCع. Post-hoc comparison between control and single blast $(* \mathrm{p}<0.05)$, and between control and single blast + bryostatin-1 (** $\mathrm{p}<0.01)(\mathbf{C})$. Bryostatin-1 significantly decreased PKC $\alpha$ levels and increased PKC $\varepsilon$ levels when administered after blast exposure. 
A.

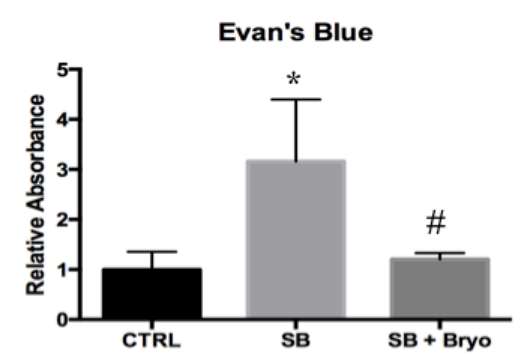

B.

CTRL

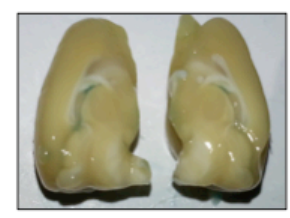

$\mathrm{SB}$

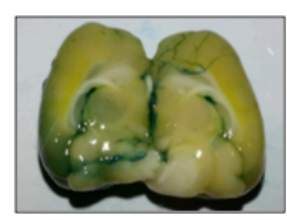

$\mathrm{SB}+\mathrm{Bryo}$

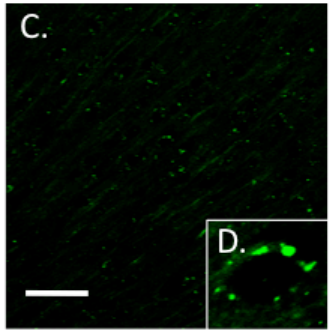

CTRL
SB
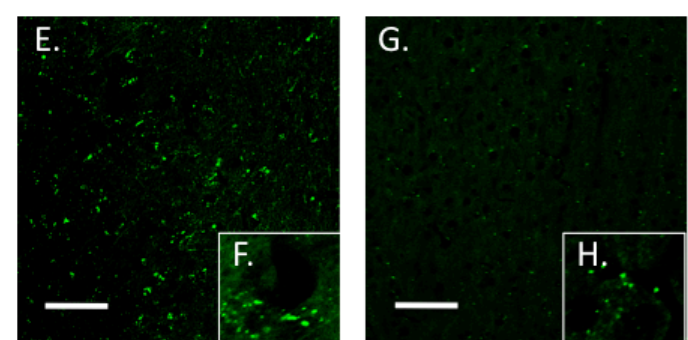

$\mathrm{SB}+$ Bryo

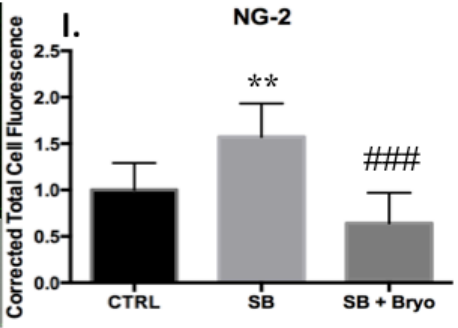

Figure 4.4: Bryostatin-1 preserves BBB integrity. Evan's blue binds to albumin and is a widely used marker for detecting breaches in the BBB. NG-2 is a proteoglycan found in pericytes that will be increased when the BBB is disrupted. Scale bar $=100 \mu \mathrm{m}$ in left prefrontal cortex. A significant difference between groups was observed for EB absorbance in the brain after femoral vein injection post-blast. Post-hoc comparison revealed a significant difference between control and single blast $\left({ }^{*} \mathrm{p}<0.05\right)$, and between single blast and single blast + bryostatin-1 ( $\# \mathrm{p}<0.05)(\mathbf{A})$. Gross examination revealed increased EB staining in the left hemisphere following blast exposure that was decreased when bryostatin-1 was given following blast $(\mathbf{B})$. A significant difference between groups was observed for NG-2 IHC fluorescent staining (C-H). Post-hoc comparison revealed a significant difference between control and single blast $(* * p<0.01)$, and between single blast and single blast + bryostatin-1 (\#\#\# p<0.001) (I). 
A.

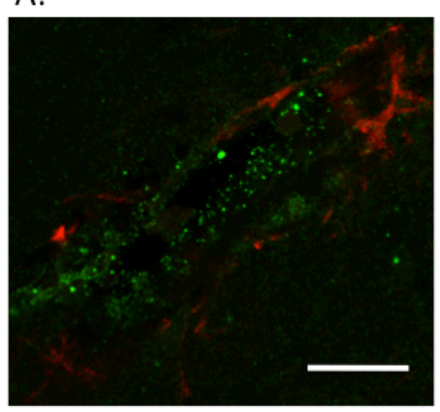

CTRL

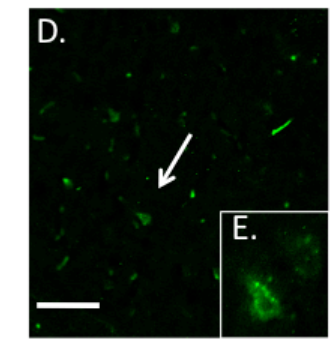

CTRL

CTRL
B.

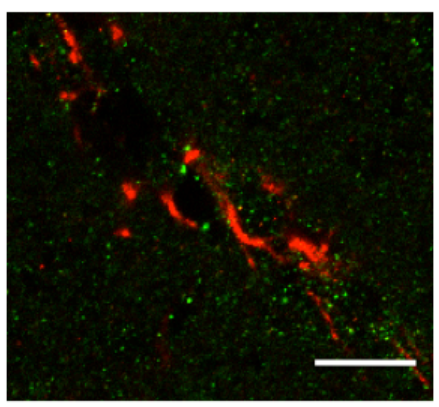

SB

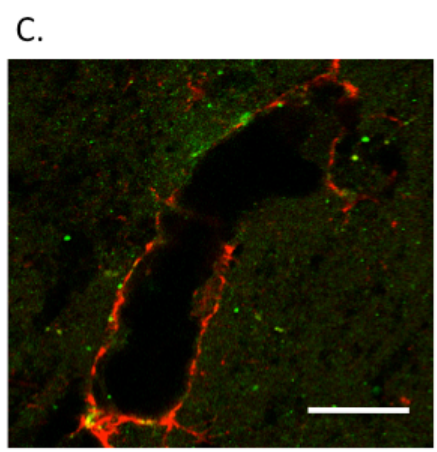

SB + Bryo

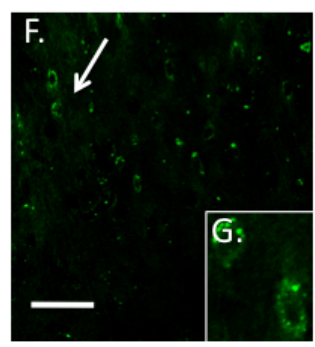

SB

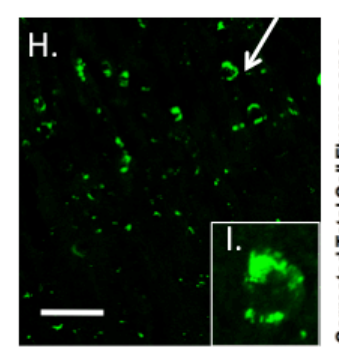

$\mathrm{SB}+$ Bryo

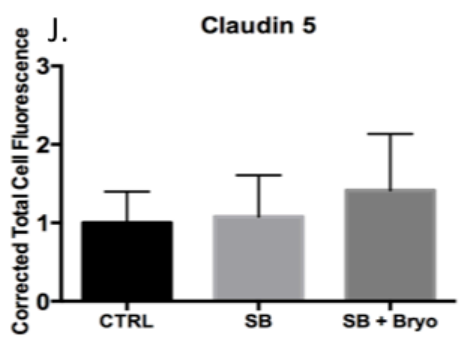

SB +

Figure 4.5: Vasculature disruption after blast exposure is independent of claudin-5 regulation. PKC $\alpha$ specifically regulates occludin, ZO-1, and VE-Cadherin, but not claudin-5. Claudin-5 levels may therefore be independent of bryostatin-1 modulation. Scale bar $=5 \mu \mathrm{m}$ in the left prefrontal cortex $(\mathbf{A}-\mathbf{C})$. Scale bar $=100 \mu \mathrm{m}$ in the left prefrontal cortex (D-J). VWF (green) was co-localized with GFAP (red) to give a visual representation of cerebral vasculature. Control vasculature in the left prefrontal cortex was visibly intact (A). 24 hours after blast exposure left prefrontal cortex vasculature was visibly disrupted as indicated by the sparse VWF staining (B). Bryostatin-1 preserved vasculature integrity when administered after blast exposure (C). No significant differences were observed between groups for claudin-5 using fluorescent IHC (D-J). 
A.
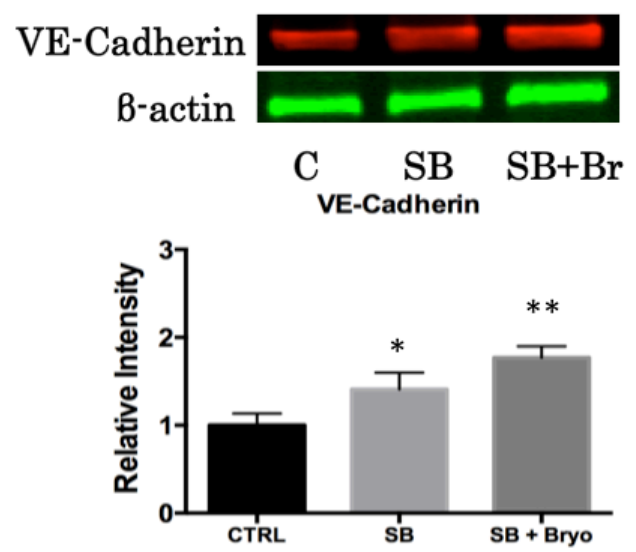

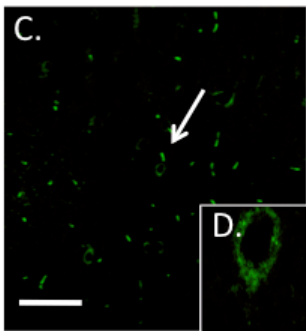

CTRL

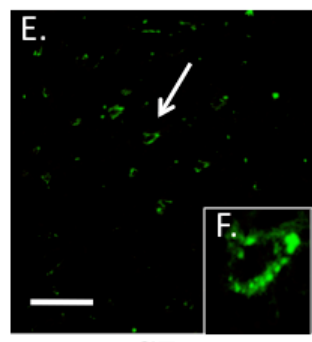

SB
B.
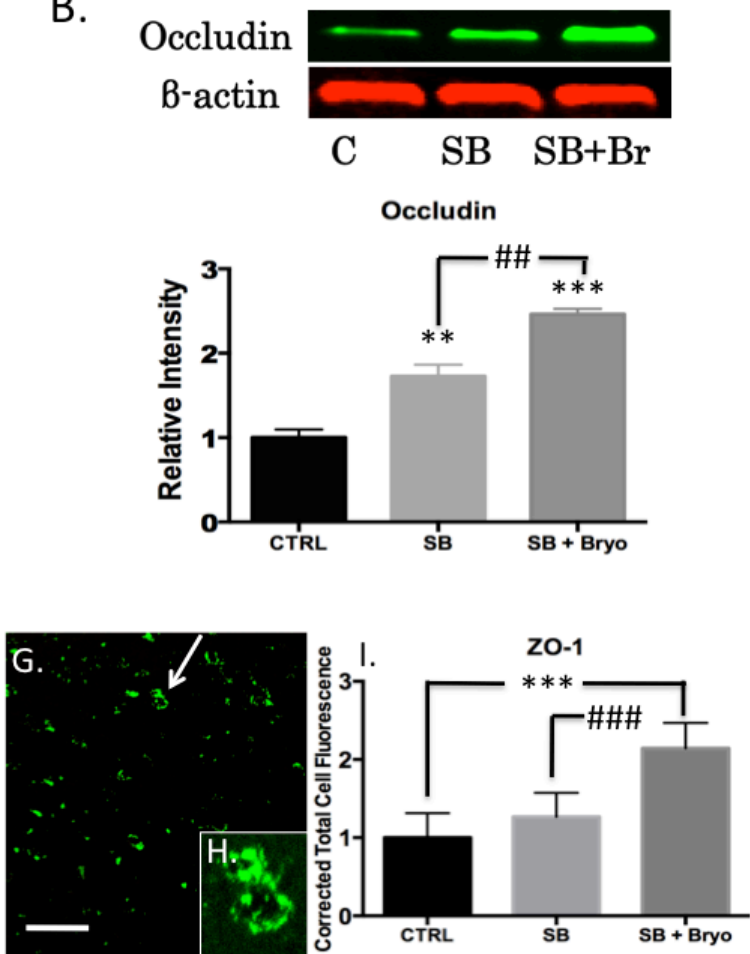

SB + Bryo

Figure 4.6: Bryostatin-1 significantly increases tight junction proteins. Our data show that bryostatin-1 significantly upregulated tight junction proteins leading to maintenance of BBB integrity following blast TBI. Scale bar $=100 \mu \mathrm{m}$ in left prefrontal cortex. A significant difference was observed between groups using western blot for VE-Cadherin with post hoc comparison showing significance between control and single blast (* $\mathrm{p}<0.05)$, and between control and single blast + bryostatin- $1(* * p<0.01) 24$ hours after blast exposure (A). A significant difference was observed between groups using western blot for occludin with post hoc comparison showing significance between control and single blast $(* * \mathrm{p}<0.01)$, between control and single blast + bryostatin- $1(* * * \mathrm{p}<0.001)$, and between single blast and single blast + bryostatin-1 (\#\# $p<0.01) 24$ hours after blast exposure (B). A significant difference was observed between groups using fluorescent IHC for ZO-1 with post hoc comparison showing significance between control and single 
blast + bryostatin-1 $(* * * \mathrm{p}<0.001)$, and between single blast and single blast + bryostatin1 (\#\#\# p<0.001) 24 hours after blast exposure $(\mathbf{C})$. 

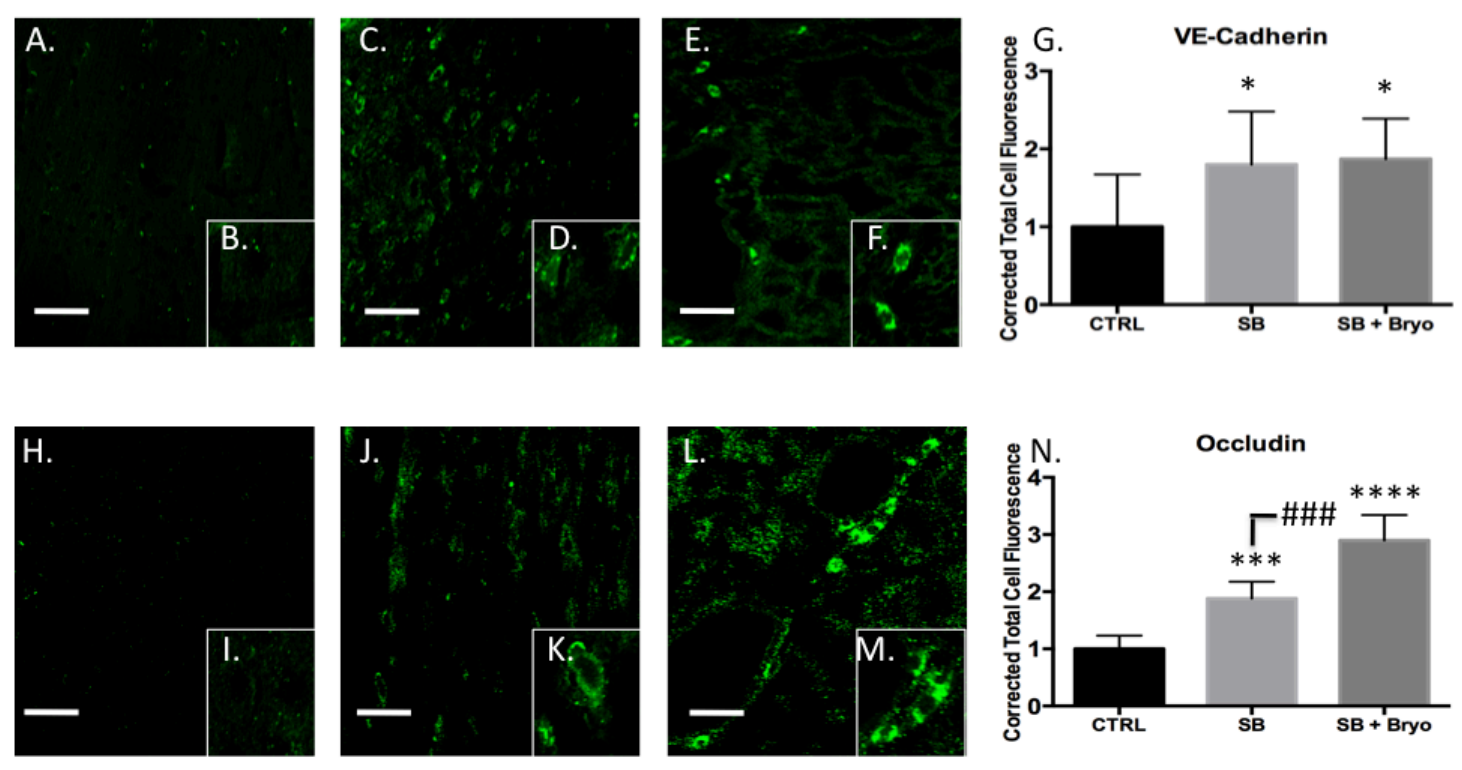

CTRL

SB

$$
\mathrm{SB}+\text { Bryo }
$$

Figure 4.7: Tight junction protein expression was increased by bryostatin-1 at vasculature. Tight junction protein return of function is necessary for restoration of BBB integrity. Scale bar $=75 \mu \mathrm{m}$ in left prefrontal cortex. A significant difference was observed between groups using fluorescent IHC for VE-Cadherin with post hoc comparison showing significance between control and single blast $(* \mathrm{p}<0.05)$, and between control and single blast + bryostatin-1 $(* p<0.05) 24$ hours after blast exposure (A-G). A significant difference was observed between groups using fluorescent IHC for occludin with post hoc comparison showing significance between control and single blast $(* * * \mathrm{p}<0.001)$, between control and single blast + bryostatin-1 $(* * * * \mathrm{p}<0.0001)$, and between single blast and single blast + bryostatin-1 (\#\#\# $\mathrm{p}<0.001) 24$ hours after blast exposure (H-N). 

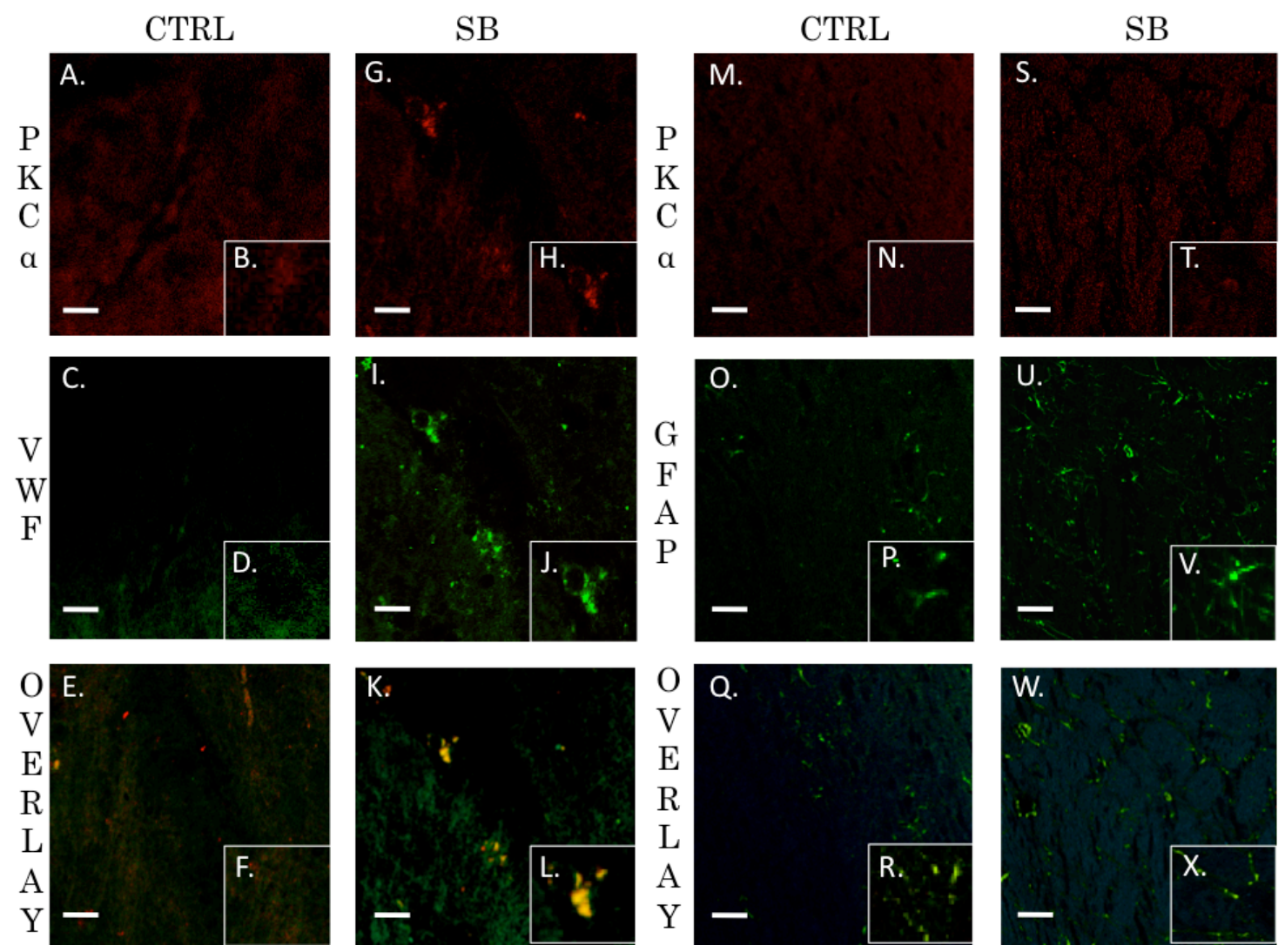

Figure 4.8: PKC $\alpha$ co-localized with endothelial cells but not astrocytes. Both astrocytes and endothelial cells are critical for maintenance of the BBB. PKC $\alpha$ activity within endothelial cells plays an intimate role in regulating extracellular tight junction proteins. Fluorescnt IHC red staining for PKC $\alpha$, green staining for VWF (endothelial) or GFAP (astocyte), and yellow is overlay. Scale bar $=20 \mu \mathrm{m}$ in left prefrontal cortex. PKC $\alpha(\mathbf{A})$ with inlay (B) and VWF (C) with inlay (D) have a weak overlay with a Pearson's coefficient of $\mathrm{r}=0.395$ seen in $(\mathbf{E})$ with inlay $(\mathbf{F})$ for control animals. PKC $\alpha(\mathbf{G})$ with inlay (H) and VWF (I) with inlay $(\mathbf{J})$ have a very strong overlay with an overlap coefficient of $\mathrm{r}=0.954$ seen in $(\mathbf{K})$ with inlay $(\mathbf{L}) 24$ hours post blast exposure. PKC $\alpha(\mathbf{M})$ with inlay $(\mathbf{N})$ and GFAP $(\mathbf{O})$ with inlay $(\mathbf{P})$ have a weak overlay with a Pearson's coefficient of $r=0.351$ seen in $(\mathbf{Q})$ with inlay $(\mathbf{R})$ for control animals. PKC $\alpha(\mathbf{S})$ with 
inlay (T) and GFAP (U) with inlay (V) have a weak overlay with an overlap coefficient of $r=0.379$ seen in $(\mathbf{W})$ with inlay $(\mathbf{X}) 24$ hours post blast exposure. 

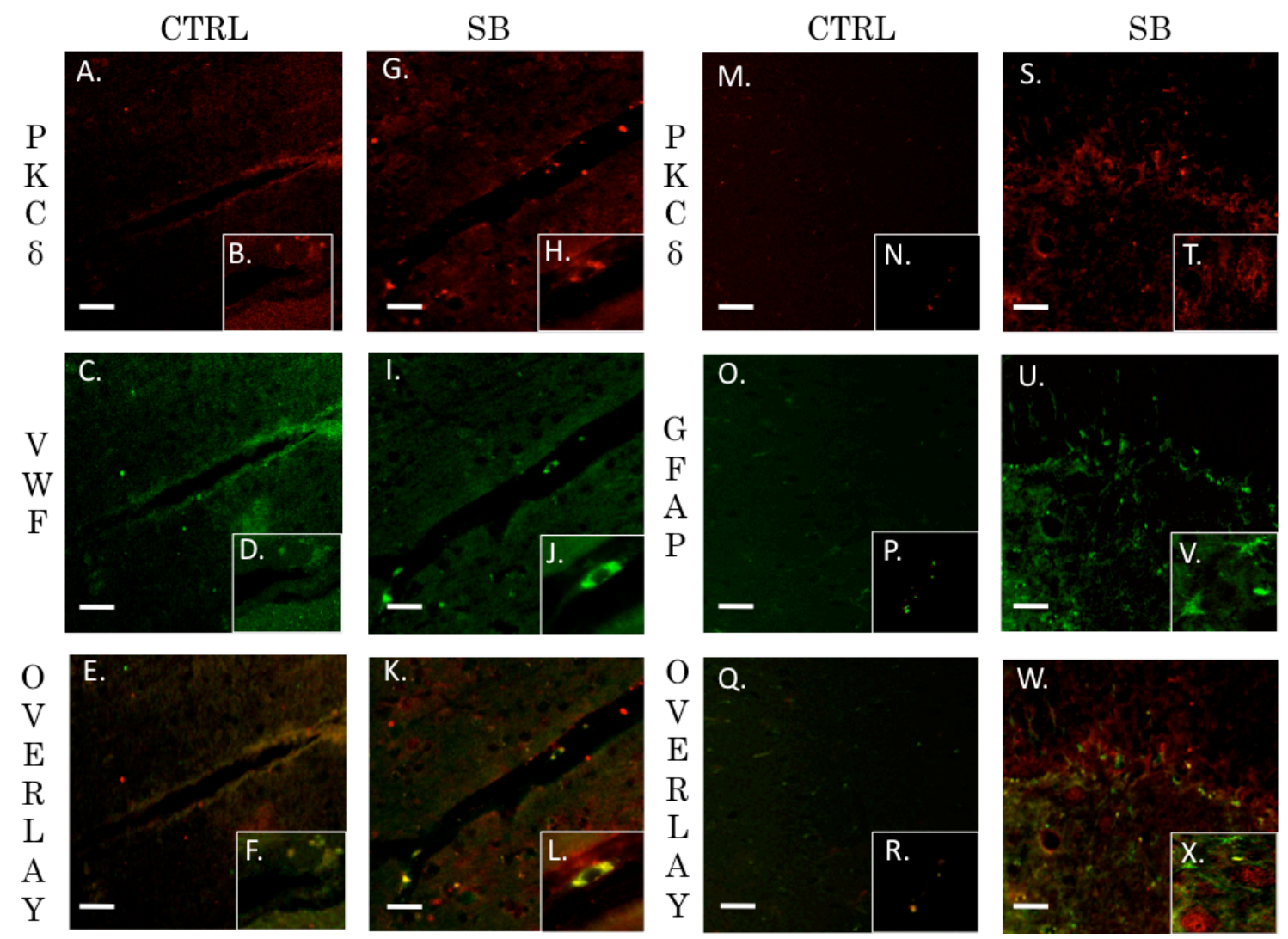

Figure 4.9: PKC $\delta$ co-localized with endothelial cells but not astrocytes. PKC $\delta$ plays an important role in mediating vascular tone. Its role in regulation of tight junction proteins is not completely understood. Fluorescent IHC red staining for PKC $\mathrm{B}$, green staining for VWF (endothelial) or GFAP (astocyte), and yellow is overlay. Scale bar $=20 \mu \mathrm{m}$ in left prefrontal cortex. PKCס (A) with inlay (B) and VWF (C) with inlay (D) have a moderate overlay with a Pearson's coefficient of $r=0.61$ seen in $(\mathbf{E})$ with inlay $(\mathbf{F})$ for control animals. PKC $(\mathbf{G})$ with inlay $(\mathbf{H})$ and $\operatorname{VWF}(\mathbf{I})$ with inlay $(\mathbf{J})$ have a strong overlay with an overlap coefficient of $\mathrm{r}=0.88$ seen in $(\mathbf{K})$ with inlay $(\mathbf{L}) 24$ hours post blast exposure. PKC $(\mathbf{M})$ with inlay $(\mathbf{N})$ and GFAP $(\mathbf{O})$ with inlay $(\mathbf{P})$ have a very weak overlay with a Pearson's coefficient of $\mathrm{r}=0.029$ seen in $(\mathbf{Q})$ with inlay $(\mathbf{R})$ for control animals. PKCס (S) 
with inlay (T) and GFAP (U) with inlay (V) have a moderate overlay with an overlap coefficient of $\mathrm{r}=0.449$ seen in $(\mathbf{W})$ with inlay $(\mathbf{X}) 24$ hours post blast exposure. 

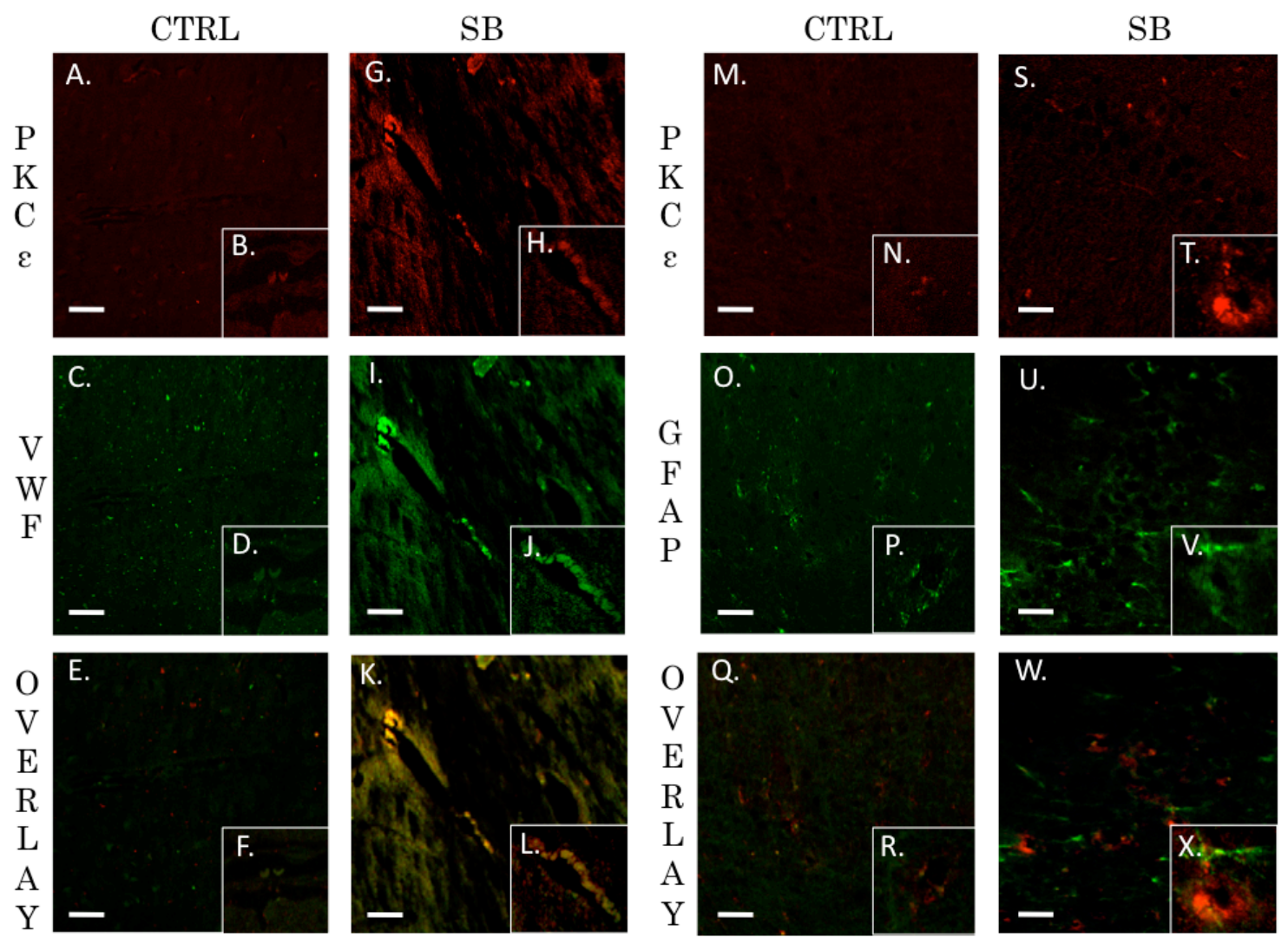

Figure 4.10: $\mathrm{PKC} \varepsilon$ co-localized with endothelial cells but not astrocytes. PKC $\varepsilon$ contributes to neuroprotection when increased after brain injury. PKC $\varepsilon$ has been associated with improved cognitive performance and decreased neurodegeneration. Fluorescent IHC red staining for PKCE, green staining for VWF (endothelial) or GFAP (astrocyte), and yellow is overlay. Scale bar $=20 \mu \mathrm{m}$ in left prefrontal cortex. PKCE (A) with inlay (B) and VWF (C) with inlay (D) have a very weak overlay with a Pearson's coefficient of $\mathrm{r}=0.004$ seen in $(\mathbf{E})$ with inlay $(\mathbf{F})$ for control animals. PKCE $(\mathbf{G})$ with inlay (H) and VWF (I) with inlay (J) have a strong overlay with an overlap coefficient of $\mathrm{r}=0.978$ seen in $(\mathbf{K})$ with inlay $(\mathbf{L}) 24$ hours post blast exposure. PKC $\varepsilon$ (M) with inlay $(\mathbf{N})$ and GFAP $(\mathbf{O})$ with inlay $(\mathbf{P})$ have a weak overlay with a Pearson's coefficient of $\mathrm{r}=0.133$ seen in $(\mathbf{Q})$ with inlay $(\mathbf{R})$ for control animals. PKCE $(\mathbf{S})$ with inlay $(\mathbf{T})$ and 
GFAP ( $\mathbf{U})$ with inlay $(\mathbf{V})$ have a weak overlay with an overlap coefficient of $r=0.394$ seen in (W) with inlay (X) 24 hours post blast exposure. 

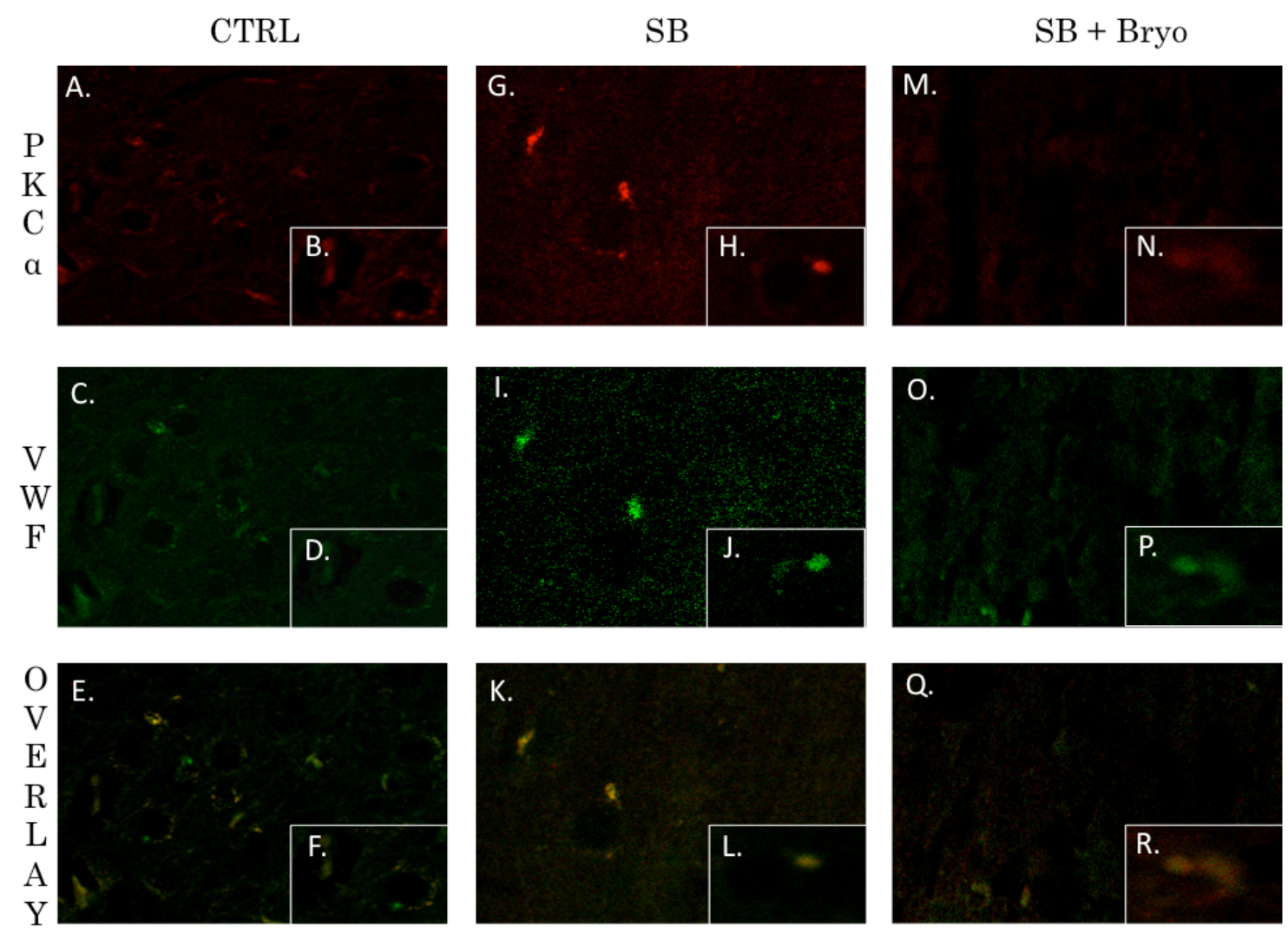

Supplementary Figure 4.1: Blast exposed animals have greater number of cells colocalized with PKC $\alpha$ and VWF. Staining for groups: red=PKC $\alpha$, green $=\mathrm{VWF}$, and yellow $=$ overlay. Regions analyzed for ratio of positive cells to total cells. Representative section for control group PKCa (A) with inlay (B), VWF (C) with inlay (D), and overlay (E) with inlay $(\mathbf{F})$. Representative section for single blast exposed group PKC $\alpha(\mathbf{G})$ with inlay (H), VWF (I) with inlay (J), and overlay $(\mathbf{K})$ with inlay (L). Representative section for single blast + bryostatin-1 group PKCa (M) with inlay (N), VWF (O) with inlay (P), and overlay $(\mathbf{Q})$ with inlay $(\mathbf{R})$. 


\section{Chapter Five}

\section{Endoplasmic reticulum stress implicated in chronic traumatic encephalopathy}

This work is published in J Neurosurg. 2016 Mar;124(3):687-702. doi:

10.3171/2015.3.JNS141802. Epub 2015 Sep 18. PMID: 26381255

Lucke-Wold BP, Turner RC, Logsdon AF, Nguyen L, Bailes JE, Lee JM, Robson MJ, Omalu BI, Huber JD, Rosen CL 


\begin{abstract}
Object: Chronic traumatic encephalopathy is a progressive neurodegenerative disease characterized by neurofibrillary tau tangles following repetitive neurotrauma. The underlying mechanism linking traumatic brain injury to chronic traumatic encephalopathy has not been elucidated. We investigate the role of endoplasmic reticulum stress as a link between acute neurotrauma and chronic neurodegeneration.
\end{abstract}

Methods: We used pharmacological, biochemical, and behavioral tools to assess the role of endoplasmic reticulum stress in linking acute repetitive traumatic brain injury to the development of chronic neurodegeneration. Data from our clinically relevant and validated rodent blast model was compared to post-mortem human chronic traumatic encephalopathy pathology from a National Football League player and World Wrestling Entertainment wrestler.

Results: Our results demonstrate strong correlation of endoplasmic reticulum stress activation with subsequent tau hyperphosphorylation. Various endoplasmic reticulum stress markers were increased in human chronic traumatic encephalopathy specimens, and the endoplasmic reticulum stress response was associated with an increase in the tau kinase, glycogen synthase kinase three $\beta$. Docosahexaenoic acid, an endoplasmic reticulum stress inhibitor, improved cognitive performance in our rat model 3 weeks after repetitive blast exposure. Our data show that docosahexaenoic acid administration substantially reduced tau hyperphosphorylation $(\mathrm{t}=4.111, P<0.05)$, improved cognition $(\mathrm{t}=6.532, P<0.001)$, and inhibited endoplasmic reticulum stress activation $(\mathrm{t}=5.631, P$ 
$<0.01$ ), and for the first time that endoplasmic reticulum stress is involved in the pathophysiology of chronic traumatic encephalopathy.

Conclusion: Docosahexaenoic acid therefore warrants further investigation as a potential therapeutic for the prevention of chronic traumatic encephalopathy.

KEY WORDS: chronic traumatic encephalopathy, blast traumatic brain injury, endoplasmic reticulum stress, hyperphosphorylated tau, docosahexaenoic acid

\section{INTRODUCTION}

Chronic traumatic encephalopathy (CTE) is a progressive tau-dependent neurodegenerative disease associated with a history of concussive and/or subconcussive injury in sports or from exposure to blast waves in military personnel (Stein et al 2014). Several progressive neuropsychiatric symptoms such as impulsive behavior, depression, loss of executive function, and cognitive decline have been reported with this progressive tauopathy (McKee et al 2014). Alarmingly, the number of sports-related concussions has risen to three-hundred-thousand per year in the United States, and the number of military service personnel exposed to blast traumatic brain injury (TBI) during the recent wars in Iraq and Afghanistan was estimated at two-hundred-seventy thousand (Noble \& Hesdorffer 2013, Sosa et al 2013). Although several case reports and case series have examined the underlying pathology of CTE, few studies have looked at pathways that link TBI to chronic neurodegeneration (McKee et al 2010, Omalu et al 2010a, Omalu et al 2010b, Watanabe et al 2010). As such, the pathophysiology of CTE development remains poorly understood and no viable therapeutics are under development due to the lack of identified therapeutic targets (Yokobori et al 2013). To address this lack of 
understanding, we investigated one component of the pathophysiology linking acute neurotrauma to chronic neurodegeneration.

We propose that the endoplasmic reticulum (ER) stress response may play an important role in linking acute neurotrauma to chronic tau-dependent neurodegeneration (Lucke-Wold et al 2014c). The ER stress response consists of three arms: protein kinase RNA-like ER kinase (PERK), inositol requiring enzyme one $\alpha$ (IRE1 $\alpha$ ), and activating transcription factor six (ATF6). The arms are activated when binding immunoglobulin protein (BiP) dissociates from the ER membrane signaling an increase in unfolded proteins (Kang et al 2013). While arm one (PERK) is generally neuroprotective, it can elevate $\mathrm{C} / \mathrm{EBP}$ homology protein (CHOP) above threshold at extended time points leading to apoptosis (Hiramatsu et al 2014). Arm two (IRE1 $\alpha$ ) and arm three (ATF6) likewise increase CHOP and trigger pro-apoptotic pathways (Shoulders et al 2013). Arm two can also lead to the phosphorylation of c-Jun N-terminal kinase (JNK) (Zhu et al 2014). ER stress markers are acutely increased following TBI in rodent models (Larner et al 2004). Recent findings have shown that ER stress increases following cortical contusion in a rat model with changes in amyloid precursor protein and tau (Begum et al 2014). Weight-drop TBI has been shown to increase intracellular calcium and result in the activation of the ER stress response in mice (Rubovitch et al 2011). Controlled cortical impact also increases ER stress and triggers apoptosis in rats acutely after injury (Farook et al 2013). While these studies demonstrate the role of ER stress in acute pathophysiological events after TBI, how ER stress contributes to persistent neurodegeneration remains unclear. Abisambra and colleagues have ascertained that tau from neurofibrillary tangles perpetually activates the ER stress response (Abisambra et al 
2013). Conversely the ER stress pathway can activate tau kinases such as apoptosis signal regulating kinase one, JNK, and glycogen synthase kinase three Beta (GSK3 $\beta$ ) (Chen et al 2011, Placido et al 2014, Song et al 2014). These important co-stimulatory interactions connect ER stress in interplay with chronic neurodegenerative diseases. In addition, phosphorylated-PERK has been co-localized with tau in diseases such as Alzheimer's disease and supranuclear palsy (Stutzbach et al 2013).

Herein, we provide compelling evidence that the ER stress response is not only activated acutely following neurotrauma but is involved in the development and progression of CTE-like findings in an animal model and post-mortem specimens previously described as having CTE (Omalu et al 2010b). Furthermore, we show that the development of CTE-like findings following repetitive concussive blast in rats is prevented by administration of docosahexaenoic acid (DHA). DHA, an important component of omega three fatty acids, has recently been shown to decrease ER stress by lowering intracellular calcium (Begum et al 2014). Our data show that DHA administration decreases tau kinases, tau hyperphosphorylation, and improves cognitive performance 3 weeks after repetitive blast injury. Modulation of ER stress responses may therefore represent a promising preventative and therapeutic strategy in the field of neurotrauma.

\section{METHODS}

\section{Animals and human samples}

All experiments involving animals were approved by the Institutional Animal Care and Use Committee of West Virginia University and were performed according to the 
principles of the Guide for the Care and Use of Laboratory Animals. Eighty-eight (88) young-adult male rats (Hilltop Lab Animals, Inc.) were used for this study based on power analysis calculations (DSS Research). We used twenty-four (24) rats for Morris water maze behavioral testing, thirty-two (32) rats for immunohistochemistry (IHC) staining, and thirty-two (32) rats for western blot analysis. Human sections from the entorhinal cortex of a National Football League (NFL) player with diagnosed CTE, World Wrestling Entertainment (WWE) wrestler with diagnosed CTE, and age-matched (44-year-old male) control were used for immunohistochemical studies.

The case of the WWE wrestler was previously documented and published in 2010 (Omalu et al 2010b). Briefly, he was 40 years of age at the time of death. Death was ruled a suicide with the manner of death by asphyxiation resulting from hanging. The patient had a history of repetitive brain trauma that included a car collision and mild traumatic brain injury at 6 years of age and at least fifteen self-reported concussions throughout his career in wrestling. Prior to his death, he suffered from depression and memory loss. The patient also had a history of presumed anabolic steroid use and suspected prescription drug dependency (narcotics).

The case of the NFL player was previously documented and published in 2010 (Omalu et al 2010a). Briefly, he was 36 years of age at time of death. Death was the result of a head on motor vehicle collision with a tanker truck. The patient had a history of repetitive head trauma from 4 years of collegiate football and eight seasons in the NFL. Prior to his death, he suffered from cognitive and neuropsychiatric symptoms. The patient also had a history of parasuicidal behavior. 
Experimental groups, blast exposure, and drug treatments

Groups for 24-hour post-injury IHC study: control, single blast, and repeat blast. Groups for 24-hour post- injury western blot study: control, single blast, single blast + DHA, and DHA alone. Groups for 3-week post-injury western blot and IHC study: control, repeat blast, and repeat blast + DHA. Groups for 3-week post-injury behavioral study: control, repeat blast, and repeat blast + DHA. All groups receiving repeat blast exposure had one blast administered every other day for two weeks (six total blasts). DHA (Zone Labs Inc.) was delivered i.p. $16 \mathrm{mg} / \mathrm{kg} 5$ minutes following each blast exposure for the DHA + repeat blast groups, and subsequently every other day until Morris water maze testing (Begum et al 2014).

\section{Blast exposure}

A scaled moderate intensity blast wave(s) ( $\sim 50.72$ PSI peak reflected over pressure) was released perpendicular to the right side of the animal's head with a shield of polyvinyl chloride protecting the body. Blast exposures were generated using a novel shock tube described in our prior work (Turner et al 2013). The shock tube was designed with a short driver section in order to generate a short-duration wave, a characteristic deemed clinically relevant based on extensive work demonstrating scaling principles associated with blast across species. The overpressure was recorded using piezoelectric sensors (Model 102AO5; PCB Piezotronics) placed in the reflected and incident positions at the exit of the shock tube. Data was then recorded using a sensor signal conditioner (482C Series; PCB Piezotronics) and a data acquisition board (DAQ 23GF; National Instruments). Data was then recorded on the computer using a customized program 
written with LabView version 12.0 (National Instruments). A representation of the experimental setup can be seen in supplementary figure 5.1 .

\section{Biochemistry preparation}

Animals used for western blot analysis were sacrificed by decapitation with a sharpened guillotine after being anesthetized by inhalation of $4 \%$ isoflurane (Halocarbon). For western blot, the brains were immediately placed in a lysate buffer containing: (10 mM Tris, $\mathrm{pH} 7.4,320 \mathrm{mM}$ sucrose, 1\% Triton X-100, 1\% CHAPS and $0.025 \% \mathrm{NaN}_{3}$ ) with phosphatase and protease inhibitors (1 mM EDTA, 1 mM EGTA, 50 $\mathrm{mM}$ sodium fluoride, $2 \mathrm{mM}$ sodium orthovanadate, $0.1 \mathrm{mM}$ ammonium molybdate, 0.2 $\mathrm{mM}$ phenylarsine oxide, and Roche Complete tablets) (Plattner et al 2006). Bilateral dissections of cortex, hippocampus, striatum, and cerebellum were performed, and tissue was subsequently flash frozen in liquid nitrogen and stored at $-80^{\circ} \mathrm{C}$ for current and future studies. Animals used for immunohistochemistry were anesthetized with 4\% isoflurane (Halocarbon) and transcardially perfused with ice-cold $0.9 \%$ saline followed by $10 \%$ formalin for a total of $10 \mathrm{~min}$. The brains were dissected and placed in $10 \%$ formalin. Brains were block-sectioned into 4 coronal sections, paraffin-embedded, and serially sliced with a Leica RM2235 microtome (Leica Microsystems, Wetzlar, Germany) for immunofluorescent, diaminobenzadine, and hematoxylin and eosin staining.

\section{Western blot}

Western blot procedures were performed similar to previous work published by our lab (Dinapoli et al 2010). Hippocampal protein samples were dissolved in $0.5 \mathrm{~mL}$ hot $\left(85-95^{\circ}\right.$ C) $1 \%$ sodium docecyl sulfate (SDS), sonicated, and assayed with a 
Bicinchoninic Acid (BCA) protein assay kit (Thermo Fisher Scientific, Rockfield, IL). 30 $\mu \mathrm{g}$ of protein per well was run with $2 \mathrm{X}$ Lammeli buffer on pre-cast Bolt ${ }^{\circledR}$ Bis-Tris Plus 10\% 12-well gels (Life Technologies, Carlsbad, CA) using a Bolt ${ }^{\circledR}$ Mini tank system (Life Technologies). A SeeBlue ${ }^{\circledR}$ Plus2 pre-stained Standard (Life Technologies) was used. Nitrocellulose membranes (Bio-Rad, Contra Costa, CA) were used for wet transfer (Bio-Rad) at 60 volts for 2.5 hours. Imaging was conducted with a LI-COR fluorescent scanner at wavelength 700 or 800 , intensity 6.0 , and resolution eighty-four. Images were converted to gray scale and analyzed after background subtraction (Odyssey Processing Software). Values were normalized to $\beta$-actin to give relative overall intensity.

The following primary antibodies were used: AT270, growth arrest and DNA damage-inducible protein 34 (GADD34) (Thermo), BiP, CHOP, p-JNK (Cell Signaling), p-GSK3 $\beta$ (Santa Cruz), and CP-13 (kindly gifted by Dr. Peter Davies, Albert Einstein College of Medicine) all at a concentration of 1:1000. Anti-rabbit or anti-mouse IRDye secondary antibodies (LI-COR) were used at a concentration of 1:2000. A $\beta$-actin rabbit monoclonal antibody (Cell Signaling) was used as an endogenous control at a concentration of 1:10,000. Imaging was conducted with a LI-COR fluorescent scanner and analyzed after background subtraction (Odyssey Processing Software). Values were normalized to $\beta$-actin to give relative overall intensity.

\section{Immunohistochemistry}

Qualitative and quantitative staining was completed for this paper that utilized previously described techniques for hematoxylin and eosin, bright-field imaging with diaminobenzadine (DAB), and fluorescent imaging (Robson et al 2014, Turner et al 
2013). Briefly, in order to remove paraffin, slides were washed for 5 minutes in xylene, $100 \% \mathrm{EtOH}$, and $95 \% \mathrm{EtOH}$ followed by 5 minute rehydration in $\mathrm{dH}_{2} \mathrm{O}$. The slides were then quenched with $10 \%$ methanol and $10 \% \mathrm{H}_{2} \mathrm{O}_{2}$ in Dulbecco's phosphate buffered saline (DPBS) for 15 minutes. After quenching, slides were rinsed two times in DPBS for 10 minutes each. The slides were then placed in permeabilizing solution (1.8\% L-Lysine, $4 \%$ horse serum, and $0.2 \%$ Triton X-100 in DPBS) for 30 minutes. For fluorescent staining, brain slices were circumscribed, incubated with primary antibody overnight followed by a fluorescent secondary for three hours, and fixed with a coverslip. If staining for co-localization, a second set of primary and secondary antibody was applied prior to fixing the coverslip. For diaminobenzadine staining, primary antibody, secondary antibody, strepavidin-HRP, and then DAB was applied before fixing with the coverslip. The hematoxylin and eosin protocol consisted of dissolving off paraffin with 5 minute washes in xylene, $100 \% \mathrm{EtOH}$, and $95 \% \mathrm{EtOH}$. The slides were then stained in Harris Hematoxylin Solution for 2 minutes and 15 seconds. The slides were rinsed in running tap water and then dipped in differentiating solution $(0.25 \mathrm{~mL}$ concentrated $\mathrm{HCl}$ in 100 $\mathrm{mL}$ of $70 \% \mathrm{EtOH})$. The slides were rinsed again in running tap water and placed in Scott's Tap Water substitute ( $2 \mathrm{~g}$ of $\mathrm{NaHCO}_{3}$ and $20 \mathrm{~g} \mathrm{MgSO}_{4}$ in $1 \mathrm{~L}$ of tap water) for 1 minute. Slides were placed in $95 \% \mathrm{EtOH}$ for 0.5 minutes and stained with Eosin $\mathrm{Y}$ Solution for 0.5 minutes. The slides were dehydrated with 5-minute washes in $95 \%$ EtOH, $100 \% \mathrm{EtOH}$, and xylene and then a coverslip was fixed with Permount.

Primary antibodies were CHOP, IRE1 $\alpha$, x-box binding protein one (XBP1), phosphorylated eukaryotic initiation factor two alpha (p-eIF2 $\alpha$ ), p-PERK, ATF6 (Cell Signaling), AT100, AT270, GSK3 $\beta$ (Thermo), and MCI, paired helical filament (PHF), 
and CP-13 (kindly gifted by Dr. Peter Davies, Albert Einstein College of Medicine). Secondary antibodies for fluorescent imaging were Alexa Fluor 488 and 594 (Life Technologies) tagged with the corresponding species to the primary antibody. Imaging was done with a Zeiss Axio Imager two (Carl Zeiss Microscopy, Thüringen, Germany). For quantification of DAB staining, perivascular regions were randomly selected from hippocampus region for rats and entorhinal cortex for humans. An observer blinded to experimental group randomly selected 100 total cells. The number of positive cells was reported as a fraction of total cells counted. For fluorescent staining, twenty cells per slide (hippocampus rat) (entorhinal cortex human) were randomly selected by blinded observer, outlined, and measured. Fluorescent density was compared to background readings, corrected with ImageJ software, and quantified. Co-localization quantification with the Just Another Co-localization plugin for ImageJ was used to determine overlap coefficient or Pearson's coefficient (Bolte \& Cordelieres 2006). Overlap coefficient was calculated using $\mathrm{k}^{\wedge} 2=\mathrm{k} 1 * \mathrm{k} 2$ with values adjusted to threshold.

\section{Morris water maze}

Animals were assessed beginning two weeks after final blast exposure. The pool was $\sim 180 \mathrm{~cm}$ in diameter with a $10 \mathrm{~cm}$ x $10 \mathrm{~cm}$ platform submerged $2.5 \mathrm{~cm}$ below water $\left(20^{\circ} \mathrm{C}\right)$. The training paradigm consisted of 6 days of spatial acquisition with a hidden platform followed by a probe day where the platform was removed. On acquisition days, rats were placed into the maze apparatus four times from different pre-determined locations. The animals were allowed 2 minutes per trial to locate the platform. Timing stopped once platform was found. If the animal did not find the platform, it was placed on the platform for 15 seconds to facilitate acquisition before starting the next trial. On the 
probe day, animals were placed in the apparatus for 1 minute at a novel entrance point. The time the animal spent exploring an area encompassing $300 \%$ of where the platform had been was recorded. Any-maze video tracking software (Stoelting Co., Wood Dale, IL) was used to record distance traveled as well as latency to reach platform for all studies. Tracking plots for each animal were recorded (Supplementary Figure 5.2).

\section{$\underline{\text { Statistical analysis }}$}

A blinded observer performed all quantification for IHC and western blot. Oneway Analysis of Variance (ANOVA) was used for IHC, western blot, and Morris water maze. Bonferroni post-hoc comparison was used to determine differences between groups. For DAB staining, a chi-square analysis was used to compare between groups. For co-localization studies, Pearson's coefficient was obtained for control sections. Overlap coefficient was obtained for experimental groups to determine extent of samecell protein expression. Two-way ANOVA was used to determine time dependent acquisition for Morris water maze learning trials. GraphPad Prism 5.0 (GraphPad Software, Inc., La Jolla, CA) was used for statistical analysis. $P<0.05$ was considered statistically significant for all data analyzed.

\section{RESULTS}

\section{Tau pathology is evident in blast-injured animals and human CTE}

Key pathologic findings for CTE include tau hyperphosphorylation and neuritic threads. The pathology is commonly located in subcortical and perivascular foci (McKee et al 2013). DAB staining revealed increased tau markers, PHF and CP-13, after repeat blast in perivascular areas adjacent to the hippocampus in Sprague Dawley rats 3 weeks post-injury, but not in control or repeat blast + DHA animals (Figure 5.1 A-L). Ratio of 
positive PHF stained cells for control $=0 / 100$, repeat blast $=18 / 100$, and repeat blast + DHA $=3 / 100 . \quad \chi^{2}=26.571$ with 2 degrees of freedom and $p<0.0001$. Ratio of positive $\mathrm{CP}-13$ stained cells for control $=0 / 100$, repeat blast $17 / 100$, and repeat blast $+\mathrm{DHA}=$ $4 / 100 \cdot \chi^{2}=22.571$ with 2 degrees of freedom and $p<0.0001$. In addition, hematoxylin and eosin staining revealed heterogeneous appearance, chromatolysis, vacuolization, and degenerating neurons 3 weeks post repetitive blast but not in control or repeat blast + DHA animals (Figure 5.1 M-R). Likewise, DAB staining revealed increased MCI, PHF, and CP-13 in the entorhinal cortex of human subjects exposed to repeated head trauma (CTE diagnosis) as compared to age-matched control specimens without a history of head trauma (Figure 5.2 A-R). Ratio of positive MCI stained cells for control $=2 / 100, \mathrm{NFL}$ 23/100, and WWE $25 / 100 \cdot \chi^{2}=19.7$ with 2 degrees of freedom and $p<0.0001$. Ratio of positive PHF stained cells for control $=2 / 100$, NFL 29/100, and WWE 32/100. $\chi^{2}=26$ with 2 degrees of freedom and $p<0.0001$. Ratio of positive CP-13 stained cells for control $=2 / 100$, NFL 27/100, and WWE 26/100. $\chi^{2}=21.794$ with 2 degrees of freedom and $\mathrm{p}<0.0001$. $\mathrm{MCI}$, PHF, and $\mathrm{CP}-13$ mark a progression in tauopathy. $\mathrm{MCI}$ is an altered form of tau that appears after injury, whereas PHF (serine 396 and 404) is a precursor to tau oligomers, and CP-13 marks pre-tangle tau at serine 202 .

\section{ER stress is increased in blast-injured animals and human CTE}

The ER stress response is an important indicator for an injured cell with all three arms of the response modulating cell survival. Acutely the ER stress response enhances survival, but chronic activation triggers apoptosis (Hetz \& Mollereau 2014). The ER stress response has been implicated in both acute injuries, such as TBI, as well as chronic neurodegenerative disease development, such as AD (Hoozemans \& Scheper 2012). We 
examined all three arms of the ER stress pathway with IHC in both our animals exposed to single and repeat blast injury and in human CTE samples. Arm one, represented by peIF2 $\alpha$, was increased 24 hours after blast injury in the Sprague Dawley rats $(F(2,12)=$ 4.07, $P<0.05$ ) (Figure 5.3 H-N). Post-hoc comparison was significant between control vs. single blast injury $(\mathrm{t}=2.852, P<0.05)$. One-way ANOVA revealed no statistical significance for arm two, represented by XBP1, 24 hours after single or repeat blast injury in Sprague Dawley rats (Figure 5.3 O-U). The one-way ANOVA for arm three, represented by ATF6, was statistically significant $(\mathrm{F}(2,12)=4.8, P<0.05)$ for blast animals, and Bonferroni post-hoc comparison revealed a significant difference between control and repeat blast groups $(\mathrm{t}=2.710, P<0.05)$ (Figure 5.3 M-R). p-eIF2 $\alpha$ was increased in the National Football League (NFL) and World Wrestling Entertainment (WWE) CTE brains $(\mathrm{F}(2,12)=13.08, P<0.001)($ Figure 5.4 H-N). Post-hoc comparison was significant between control vs. NFL brain $(\mathrm{t}=3.798, P<0.01)$ and control vs. WWE brain $(\mathrm{t}=4.866, P<0.001)$. XBP1 was also increased in the CTE brains $(\mathrm{F}(2,12)=$ 38.55, $P<0.001$ ) (Figure 5.4 A-G). Post-hoc comparison was significant between control vs. NFL brain $(\mathrm{t}=8.647, P<0.001)$ and control vs. WWE brain $(\mathrm{t}=2.998, P<0.05)$. Furthermore, ATF6 was increased in the CTE brains $(\mathrm{F}(2,12)=9.935, P<0.01)$ (Figure 5.4 O-U). Post-hoc comparison was significant between control vs. NFL brain $(\mathrm{t}=4.369$, $P<0.01)$ and control vs. WWE brain $(\mathrm{t}=2.951, P<0.05)$. CHOP was significantly elevated in human CTE brains $(\mathrm{F}(2,12)=24.17, P<0.001)$ (Figure 5.5 A-G). Post-hoc comparison was significant between control vs. NFL brain $(t=6.849 P<0.001)$. CHOP was also significantly elevated 24 hours after blast injury in Sprague Dawley rats $(F(2,12)$ 
$=4.161, P<0.05)($ Figure $5.5 \mathrm{H}-\mathrm{N})$. Post-hoc comparison was significant between

control vs. single blast injury $(\mathrm{t}=2.875, P<0.05)$.

\section{ER stress is associated with tau hyperphosphorylation}

Tau hyperphosphorylation is an important precursor for the development of CTE (Mez et al 2013). Currently, the mechanism of CTE development is not known, but ER stress offers to be a promising candidate based on prior work in neurotrauma showing an acute elevation of ER stress and chronic elevation of ER stress in neurodegenerative disease such as Alzheimer's disease. We co-stained for ER stress activation, IRE1 $\alpha$, and tau hyperphosphorylation, AT270, in human CTE brains as well as Sprague Dawley rats exposed to blast injury (Figure 5.6). IRE1 $\alpha$ was used to represent the second arm of ER stress and has been co-localized with other tauopathies (Hoozemans \& Scheper 2012). Pearson's coefficient for human control brain was low $(\mathrm{r}=0.173)$, but overlap coefficient was high for the NFL brain $(\mathrm{r}=0.881, \mathrm{k} 1=0.548$ and $\mathrm{k} 2=1.416$ with threshold $\mathrm{A}=16$ and $\mathrm{B}=19)$ and the WWE brain $(\mathrm{r}=0.881, \mathrm{k} 1=0.615$ and $\mathrm{k} 2=1.261$ with threshold $\mathrm{A}$ $=35$ and $\mathrm{B}=20$ ). Similarly, Pearson's coefficient for the Sprague Dawley control was low $(r=0.068)$, but overlap coefficient was high for single blast $(r=0.931, k 1=0.704$ and $\mathrm{k} 2=1.231$ with threshold $\mathrm{A}=16$ and $\mathrm{B}=14)$ and repeat blast $(\mathrm{r}=0.887, \mathrm{k} 1=0.518$ and $\mathrm{k} 2=1.518$ with threshold $\mathrm{A}=21$ and $\mathrm{B}=15)$.

\section{Tau kinase activity and hyperphosphorylation is increased with repetitive blast injury}

GSK $3 \beta$ is activated by the ER stress response and contributes to chronic neurodegeneration (Hoozemans \& Scheper 2012). GSK3 $\beta$ is responsible for over one third of all tau hyperphosphorylation and can lead to neuronal apoptosis (Yang et al 
2013). We propose that ER stress activates GSK3 $\beta$ in a biphasic manner both acutely and chronically (Liu et al 2012). An initial spike in activity is seen acutely which resolves, but over time GSK3 $\beta$ activity continually rises in conjunction with neurodegeneration. GSK3 $\beta$ was co-stained with a marker for tau hyperphosphorylation, AT100, in human CTE brains and Sprague Dawley rats exposed to blast injury (Figure 5.7). AT100 is a marker of tau hyperphosphorylation at threonine 212 and serine 214 , and GSK3 $\beta$ is known to phosphorylate at those sites (Cavallini et al 2013). Pearson's coefficient for human control brain was low $(r=0.286)$, but overlap coefficient was high for the NFL brain $(\mathrm{r}=0.846, \mathrm{k} 1=1.44$ and $\mathrm{k} 2=0.497$ at threshold $\mathrm{A}=2$ and $\mathrm{B}=2)$ and WWE brain $(\mathrm{r}=0.904, \mathrm{k} 1=2.316$ and $\mathrm{k} 2=0.353$ at threshold $\mathrm{A}=2$ and $\mathrm{B}=2)$. Pearson's coefficient for Sprague Dawley control was low $(\mathrm{r}=0.374)$, but overlap coefficient was high for single blast $(\mathrm{r}=0.934, \mathrm{k} 1=0.566$ and $\mathrm{k} 2=1.543$ at threshold $\mathrm{A}=6$ and $\mathrm{B}=6)$ and repeat blast $(\mathrm{r}=0.807, \mathrm{k} 1=0.566$ and $\mathrm{k} 2=1.543$ at threshold $\mathrm{A}=6$ and $\mathrm{B}=6)$.

\section{Pharmacologic modulation of ER stress alters tau hyperphosphorylation}

24 hours post-blast we examined protein expression changes from arms 1 and 2 of the ER stress response for single blast injured animals with and without DHA. A significant difference in GADD34 protein expression was seen $(\mathrm{F}(3,12)=10.17, P<$ 0.01). Post hoc analysis revealed a significant difference between control and single blast groups $(\mathrm{t}=6.009, P<0.01)$, between single blast and single blast + DHA groups $(\mathrm{t}=$ 5.92, $P<0.01)$, and between single blast and DHA only groups $(\mathrm{t}=6.848, P<0.01)$ (Figure 5.8 A). A significant difference in p-JNK was seen $(\mathrm{F}(3,12)=9.779, P<0.01)$. Post hoc analysis revealed a significant difference between control and single blast groups $(\mathrm{t}=6.221, P<0.01)$, between single blast and single blast + DHA groups $(\mathrm{t}=$ 
6.634, $P<0.01)$, and between single blast and DHA only groups $(\mathrm{t}=6.04, P<0.01)$

(Figure 5.8 B). In addition, we looked at BiP expression changes 3 weeks post repetitive blast exposure, and how ER stress activation contributes to phosphorylation of tau kinases. A significant difference in $\mathrm{BiP}$ expression was seen $(\mathrm{F}(2,6)=8.445, P<0.05)$. Post hoc analysis revealed a significant difference between control and repeat blast groups $(\mathrm{t}=4.867, P<0.05)$, and between repeat blast and repeat blast + DHA groups $(\mathrm{t}$ $=5.185, P<0.05)($ Figure 5.9 A). A significant difference in phosphorylation of GSK3 $\beta$ at tyrosine 216 was seen $(\mathrm{F}(2,6)=31.73, P<0.001)$. Post hoc analysis revealed a significant difference between control and repeat blast groups $(\mathrm{t}=10.31, P<0.001)$, and between repeat blast and repeat blast + DHA groups $(\mathrm{t}=9.085, P<0.01)($ Figure $5.9 \mathrm{~B})$. All three arms of the ER stress response are known to increase CHOP (Hoozemans \& Scheper 2012). CHOP is an important protein linking ER stress to neurodegeneration via the activation of tau kinases. A significant difference in CHOP protein expression between groups was seen 24 hours after blast exposure $(\mathrm{F}(2,13)=9.8, P<0.01)$. Post hoc analysis revealed a significant difference between control and single blast groups $(\mathrm{t}=$ 5.29, $P<0.05)$ and between single blast and single blast + DHA groups $(\mathrm{t}=5.361, P<$ 0.01). DHA reduced CHOP back toward control levels (Figure $5.10 \mathrm{~A}$ ). AT270 is a marker of tau hyperphosphorylation at threonine 181 . The threonine 181 residue is commonly phosphorylated only when tau has translocated to the cell soma (Kester et al 2014). A significant difference in AT270 between groups was seen 3 weeks after repeat blast exposure $(\mathrm{F}(2,9)=6.451, P<0.05)($ Figure $5.10 \mathrm{~B})$. Post hoc analysis revealed a significant difference between control and repeat blast groups $(\mathrm{t}=4.81, P<0.05)$ and between repeat blast and repeat blast + DHA groups $(\mathrm{t}=4.111, P<0.05)$. CP-13 signals 
a change in tau hyperphosphorylation towards pre-tangle tau. A significant difference in $\mathrm{CP}-13$ was seen 3 weeks after repeat blast exposure $(\mathrm{F}(2,6)=13.23, P<0.01)$ (Figure 5.10 C). Post hoc analysis revealed a significant difference between control and repeat blast groups $(\mathrm{t}=5.778, P<0.05)$ and between repeat blast and repeat blast + DHA groups $(\mathrm{t}=6.714, P<0.05)$.

Repeat blast exposure induces CTE-like behavioral changes alleviated by DHA $\underline{\text { administration }}$

CTE can lead to cognitive deficits and dementia (Stein et al 2014). We sought to reproduce CTE-like behavioral changes, in addition to the pathological changes described above, using a clinically relevant blast model. Using Morris water maze, a hippocampaldependent cognitive performance test, we specifically evaluated changes post-blast in order to determine potential deficits in memory acquisition and retention (Petraglia et al 2014a). In addition, we evaluated DHA, a proposed ER stress modulator, as a potential preventative/therapeutic agent. We found DHA administration 5 minutes following each blast and subsequent every other day administration attenuated learning deficits from repeat blast exposure. A significant difference in learning across trial days $(\mathrm{F}(5,126)=$ 19.68, $P<0.001)$ and between groups $(\mathrm{F}(2,126)=38.53, P<0.001)$ was observed. Post hoc analysis revealed a significant difference between control and repeat blast groups for latency to platform on day two $(\mathrm{t}=3.633, P<0.01)$, day three $(\mathrm{t}=3.455, P<0.01)$, day four $(\mathrm{t}=4.057, P<0.001)$, day five $(\mathrm{t}=3.079, P<0.01)$, and day six $(\mathrm{t}=3.149, P<$ 0.01). A significant difference in latency to platform was also seen between repeat blast and repeat blast + DHA groups on day two $(\mathrm{t}=3.855, P<0.001)$, day three $(\mathrm{t}=3.918, P$ $<0.001)$, day four $(\mathrm{t}=4.415, P<0.001)$, day five $(\mathrm{t}=3.442, P<0.01)$, and day six $(\mathrm{t}=$ 
3.283, $P<0.01$ ) (Figure $5.10 \mathrm{D}$ ). The probe trial revealed a significant difference between groups $(\mathrm{F}(2,21)=16.17, P<0.001)$ on time exploring an area $300 \%$ of where the platform had been located prior (Figure 5.10 E). Post-hoc comparison revealed significant difference between control and repeat blast groups in time spent exploring an area $300 \%$ of platform $(\mathrm{t}=7.331, P<0.001)$ as well as between repeat blast and DHA + repeat blast $(\mathrm{t}=6.532, P<0.001)$.

\section{DISCUSSION}

ER stress has recently been implicated in chronic neurodegeneration (Moreno et al 2013). Tauopathies are the result of aggregated and mutated proteins that cause immunoexcitotoxicity, and ER stress activation (Blaylock \& Maroon 2011). The ER responds to toxic buildup of proteins by shutting down protein synthesis and inhibiting protein folding (Ron \& Harding 2012). The ER stress response may accelerate tauopathy by facilitating tau hyperphosphorylation and rapid tau aggregration that leads to neurofibrillary tangles (Hoozemans \& Scheper 2012). CTE is unique amongst tauopathies in that its onset and progression is thought to be associated with repetitive head injury (Aungst et al 2014a, Macdonald et al 2014). Currently, diagnostic and treatment options are unavailable for this disease, although improving with the use of the [18F]FDDNP PET imaging probe (Small et al 2013). The growing number of athletes and soldiers experiencing CTE, characterized by symptoms of impulsivity, cognitive decline, and depression, urges renewed focus into understanding disease mechanism and disease progression (Turner et al 2012a). It has long been postulated that TBI leads to chronic neurodegeneration (Miyauchi et al 2014, Nakagawa et al 2011, Smith et al 2012). 
Recent findings have confirmed that changes in tau, amyloid, and cognition can result from neurotrauma (Dapul et al 2013, Omalu et al 2011a, Stern et al 2011). The mechanisms underlying how these changes develop is currently unknown.

We show for the first time that ER stress is intimately associated with CTE in human clinical post-mortem specimens and in the development of CTE-like pathology and behavioral deficits in an animal model of repetitive neurotrauma. ER stress is acutely triggered by rapid changes in intracellular calcium following brain injury (Kaufman \& Malhotra 2014). All three arms of the ER stress response were significantly elevated in human CTE brains indicating that not only does neurotrauma lead to acute increases in ER stress, but also that tau pathology likely contributes to persistent elevations in ER stress during chronic neurodegeneration. CHOP, a downstream target of ER stress, was increased in the NFL CTE brain but not in the WWE CTE brain. The WWE CTE case had more severe neurofibrillary tangle pathology whereas the process of tau hyperphosphorylation was ongoing in the NFL CTE brain. Therefore, CHOP may be associated with active changes in the tau phosphorylation state. Begum and colleagues have shown that p-eIF $2 \alpha$ is increased 24 to 48 hours after TBI, but downstream components of arm two of the ER stress response were not increased until 3 to 7 days after injury (Begum et al 2014). These findings likely account for why we didn't observe a statistically significant increase in XBP1 following single and repeat blast exposure at 24 hours after injury. The other branch of arm 2, p-JNK, was however increased at 24 hours, but the expression was reduced when DHA was given. ATF6 was significantly increased 24 hours following repeat blast indicating an appropriate cleavage from the membrane due to an accumulation of unfolded proteins (Ye et al 2000). Future work will 
examine the effects of repeat blast on ER stress activation at subacute (3-7 day) time points. The CHOP increase was not statistically significant for repeat blast vs. control groups 24 hours post-injury. It is likely that CHOP will be increased after repeat blast starting 3 days post-injury. The initial lack of change is likely due to inflammatory preconditioning from the short interval between blast exposures.

Using our clinically relevant and validated blast model, we examined active changes in tau following blast induced TBI, and correlated these changes with human CTE pathology. The ER stress activated tau kinase, GSK3 $\beta$, was significantly increased after repetitive blast injury, and subsequent tau hyperphosphorylation was co-localized within the same cells where ER stress activation was occurring. The activating phosphorylation of GSK3 $\beta$ at tyrosine 216 was reduced by DHA administration. Future work will focus on teasing out exactly how an increase in CHOP may activate GSK3 $\beta$ and in which specific cell types these changes are occurring. Interestingly, ATF6 from arm three of the ER stress pathway has been shown to significantly elevate Akt (Zhou et al 2014). Since GSK3 $\beta$ triggers apoptosis, Akt is necessary in order to directly inhibit GSK3 $\beta$ and promote cell survival. Neuronal survival is required for trans-synaptic tau propagation (Pooler et al 2013). Akt is a well-known tau kinase that fosters the transsynaptic spread of tau tangles over time (Sadik et al 2009a). We plan to investigate these interactions between Akt and GSK3 $\beta$ in follow up studies. It is likely that ATF6 is continually activated at extended time points in the injury cascade compared to arm one and two of the ER stress response, which would account for the initial increase we observed in GSK3 $\beta$ following blast exposure. 
Many of the experimental drugs that target the ER stress response have high affinity for a single arm of the pathway at upstream targets. While beneficial acutely, these compounds fail to address the compensatory response of the remaining arms of the ER stress response and subsequent crosstalk between the arms. The crosstalk is what allows CHOP to be pushed above threshold and apoptosis and tau hyperphosphorylation to ensue (Hetz \& Mollereau 2014). The ER stress response is a cellular defense mechanism. If protein folding can't be restored quickly, the ER triggers the cell to undergo apoptosis. Too much activation of any arm of the ER stress response can accelerate this process and contribute to chronic neurodegeneration (Fonseca et al 2013). DHA acutely inhibits all three arms of the ER stress response after injury by limiting the intracellular calcium surge (Begum et al 2013). DHA reduced GADD34 and p-JNK acutely, but also decreased $\mathrm{BiP} 3$ weeks following repeat blast. By acting at a target step above ER stress activation and $\mathrm{BiP}$ dissociation, DHA prevents the compensatory reverberation from other arms of the ER stress pathway allowing little to no acute protein regulation resulting from injury. In addition, DHA has recently been shown to readily enter the brain and inhibit neurofibrillary tangle formation in a murine model of Alzheimer's disease (Freund Levi et al 2014, Lebbadi et al 2011). Linking these two important properties of DHA is a topic of ongoing investigation.

\section{CONCLUSION}

Markers of ER stress were increased in both Sprague Dawley rat samples and human CTE specimins. These markers were co-localized with tau pathology in hippocampal neurons. Sprague Dawley rats showed similar pretangle tau changes comparable to human CTE 3 weeks post repetitive blast. Most importantly, we show that 
the omega-3 derivative, DHA, reduced ER stress mediated CHOP expression, GADD34 expression, and p-JNK at 24 hours and additionally reduced tau hyperphosphorylation, BiP dissociation, and phosphorylation of GSK3 $\beta$ at three weeks post-injury. DHA also decreased cognitive decline measured by Morris Water Maze when given 5 minutes after each blast exposure. DHA appears to have pharmacotherapeutic potential as a preventative treatment against the development of CTE and may be a promising treatment for various other forms of neurotrauma (Hasadsri et al 2013).

\section{DISCLOSURES}

No competing interests to disclose. A Research Funding and Development Grant (RFDG) from the West Virginia University Health Sciences Center Office of Research and Graduate Education supported this work along with WVU Department of Neurosurgery. (R. C. T.) and (M. J. R.) were supported by a NIH training grants (GM08174) and (NS007491).

\section{ACKNOWLEDGEMENTS}

The authors would like to acknowledge the work done by: Nic Jordan, B.S., and Kelly Smith, B.S., for assistance with immunohistochemistry. The authors would also like to thank Dr. James O'Callaghan of CDC/NIOSH for use of resources for western blot quantification, and Diana Richardson CDC/NIOSH for tissue preparation for IHC. We also thank Dr. Rae Matsumoto for use of western blot resources in her laboratory, and the West Virginia University Microscopy Imaging Facility for assistance with microscopy. We thank James E. Robson and Peter Bennett for construction of the blast model and Dr. 
Robert Gettens and Nic St. John for design of the model. We thank Dr. Peter Davies for his kind gift of PHF, CP-13, and MCI antibodies. 

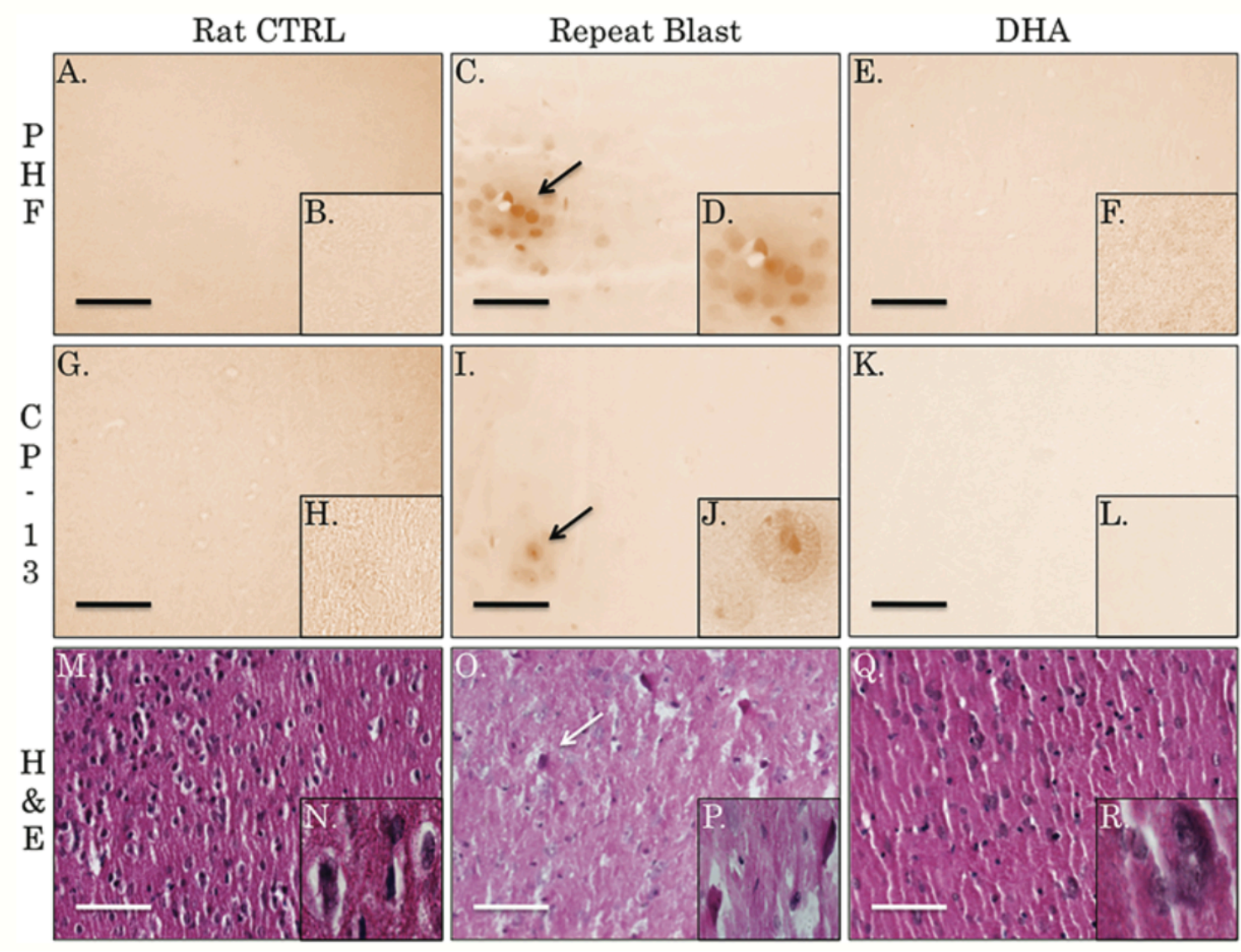

Figure 5.1: Robust tau changes 3 weeks post repetitive blast exposure. Non-transgenic rodents do not develop neurofibrillary tau tangles. Tau hyperphosphorylation can occur after traumatic neurotrauma however indicating acute changes similar to human pathology. Abbreviations: $\mathrm{CTRL}=$ control, and $\mathrm{DHA}=$ docosahexaenoic acid. In the Sprague Dawley rodent model, DAB staining revealed that PHFs were present in perivascular regions of the hippocampus for repeat blast animals (18/100) (C) 20x, scale

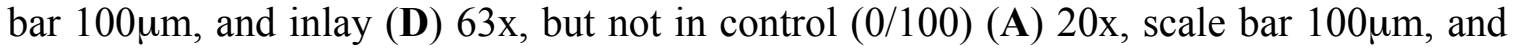
inlay (B) 63x and reduced in DHA + repeat blast animals (3/100) (E) 20x, scale bar $100 \mu \mathrm{m}$, and inlay (F) 63x. Similarly, CP-13 was increased in repeat blast animals $(17 / 100)(\mathbf{I}) 20 x$, scale bar 100 $\mu \mathrm{m}$, and inlay (J) 63x, but not in control (0/100) (G) 20x, scale bar $100 \mu \mathrm{m}$, and inlay $(\mathbf{H}) 63 \mathrm{x}$ and reduced in DHA + repeat blast animals $(4 / 100)$ 
(K) 20x, scale bar 100um, and inlay (L) 63x. In addition H\&E staining revealed nuclear chromatolysis, vacuolization, and degeneration after repeat blast (O) 20x, scale bar

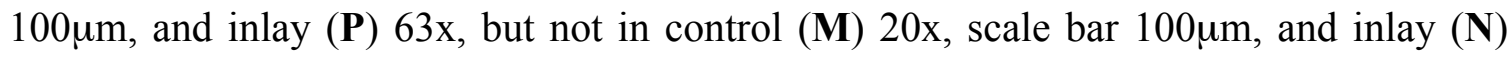
63x or repeat blast + DHA groups (Q) 20x, scale bar 100 $\mu$ m, and inlay (R) 63x. 

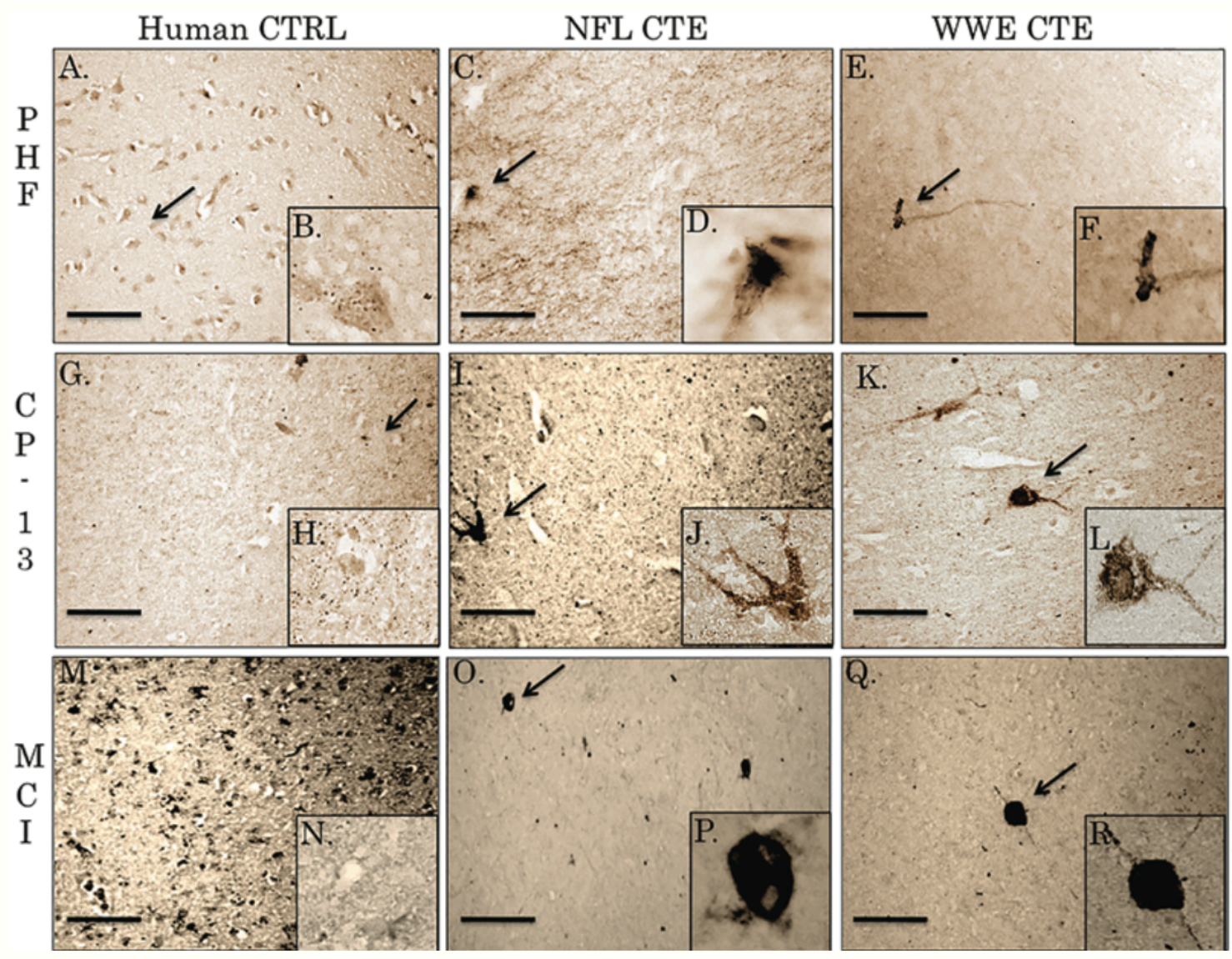

Figure 5.2: Human CTE: characterized by distinct changes in tau hyperphosphorylation. The level of phosphorylation is dependent on the structural organization of tau and additionally on tau aggregation. PHFs are precursors to tau oligomers and indicate intrasomatic accumulation of tau. Abbreviations: CTRL $=$ control, NFL $=$ National Football League, WWE $=$ World Wrestling Entertainment. DAB staining revealed that PHFs were not present in the entorhinal cortex of an age matched control (2/100) (A)

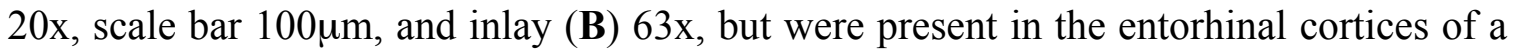
NFL player (29/100) (C) 20x, scale bar 100 $\mu$ m, and inlay (D) 63x and a WWE wrestler (32/100) (E) 20x, scale bar 100 $\mu \mathrm{m}$, and inlay (F) 63x. CP-13 is a precursor for tau neurofibrillary tangles with a balanced $3 \mathrm{R}: 4 \mathrm{R}$ ratio. $\mathrm{DAB}$ staining revealed that $\mathrm{CP}-13$ was not present in the entorhinal cortex of an age matched control (2/100) (G) 20x, scale 
bar $100 \mu \mathrm{m}$, and inlay $(\mathbf{H}) 63 \mathrm{x}$, but was present in the entorhinal cortices of a NFL player

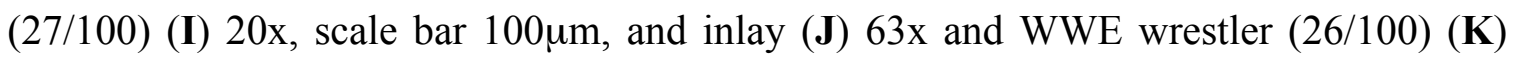

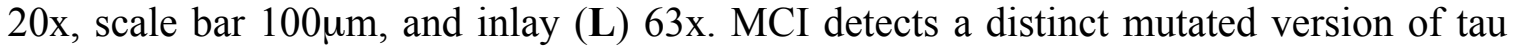
that is present in neurodegenerative tauopathies. $\mathrm{DAB}$ staining revealed no $\mathrm{MCI}$ in the entorhinal cortex of an age matched control (2/100) (M) 20x, scale bar $100 \mu \mathrm{m}$, and inlay (N) 63x, but MCI was present in the entorhinal cortices of a NFL player (23/100) (O) 20x, scale bar 100 $\mu \mathrm{m}$, and inlay (P) 63x and a WWE wrestler (25/100) (Q) 20x, scale bar $100 \mu \mathrm{m}$, and inlay $(\mathbf{R}) 63 \mathrm{x}$. 

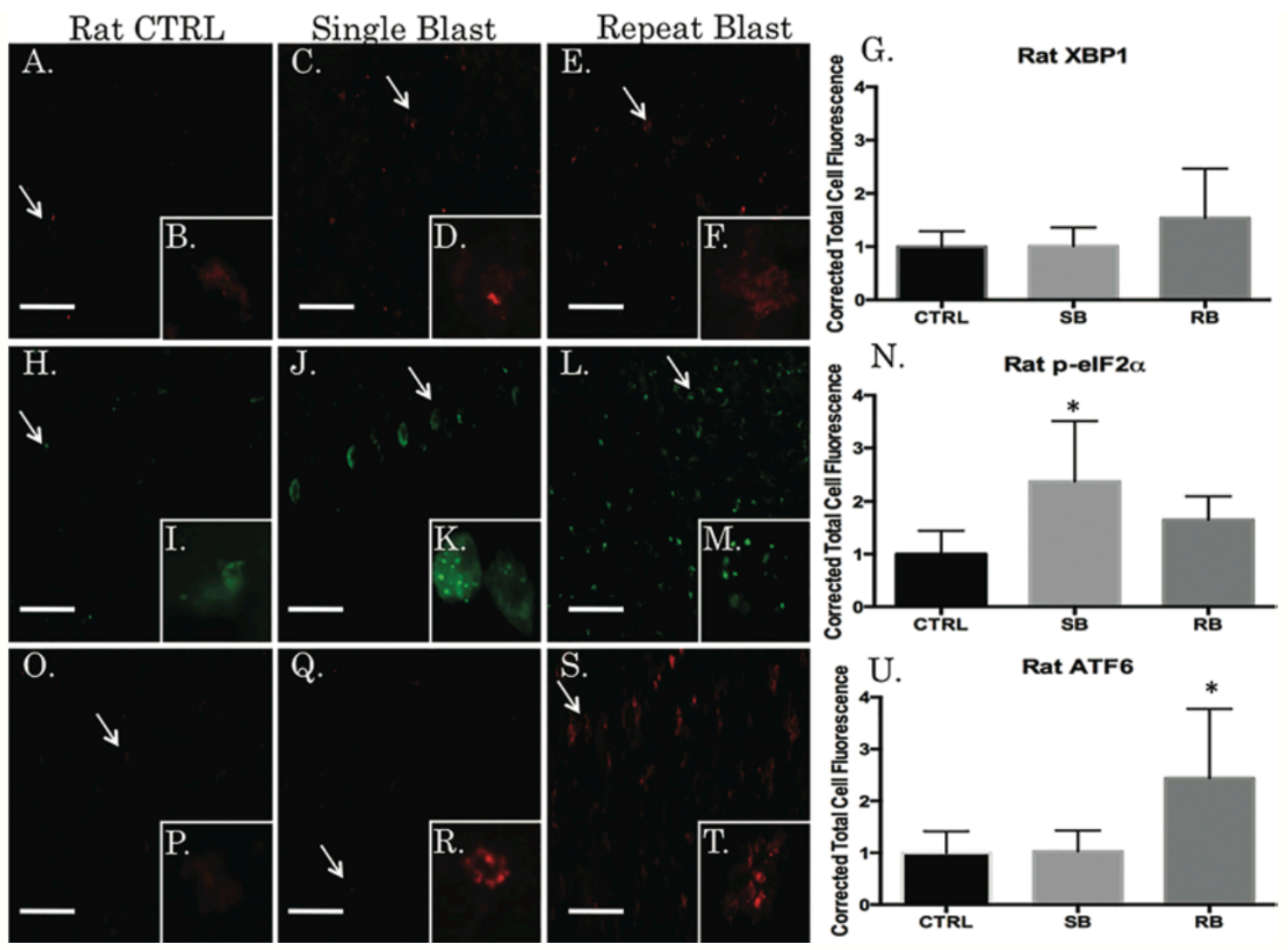

Figure 5.3: ER stress as a defense mechanism. The ER stress response is a robust cellular mechanism that can help protect the cell from injury or toxic insult and restore homeostatic processes after injury. Arm 1 in particular provides vigorous neuroprotection after trauma. While acute increases in intracellular calcium trigger a modest ER stress response, the accumulation of unfolded proteins with time can enhance the vitality of the cellular ER response incorporating the detrimental arms 2 and 3. Abbreviations: CTRL $=$ control, $\mathrm{SB}=$ single blast, and $\mathrm{RB}=$ repeat blast. $\mathrm{XBP} 1$, arm 2 of the $\mathrm{ER}$ stress response, was not significantly elevated in single blast exposed animals (C) 20x, scale bar $75 \mu \mathrm{m}$, and inlay (D) 63x or in repeat blast exposed animals (E) 20x, scale bar $75 \mu \mathrm{m}$, and inlay (F) 63x compared with control (A) 20x, scale bar 75 $\mu$ m, and inlay (B) $63 \mathrm{x}$ as depicted in the bar graph (G). P-eIF $2 \alpha$, arm 1 of the ER stress response, was significantly elevated in 
the single blast exposed animals $(P<0.05)(\mathbf{J}) 20 \mathrm{x}$, scale bar $75 \mu \mathrm{m}$, and inlay $(\mathbf{K}) 63 \mathrm{x}$ but not in the repeat blast exposed animals (L) 20x, scale bar 75um, and inlay (M) 63x compared with control (H) 20x, scale bar $75 \mu \mathrm{m}$, and inlay (I) $63 \mathrm{x}$ as depicted in the bar graph $(\mathbf{N})$. ATF6, arm 3 of the ER stress response, was not significantly elevated in single

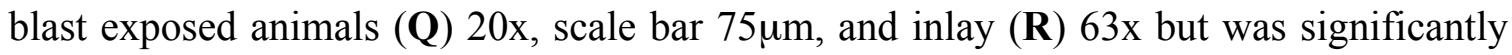
elevated in repeat blast exposed animals $(P<0.05)(\mathbf{S}) 20 \mathrm{x}$, scale bar $75 \mu \mathrm{m}$, and inlay (T) 63x compared with control (O) 20x, scale bar 75 $4 \mathrm{~m}$, and inlay (P) 63x as depicted in the bar graph $(\mathbf{U})$. 

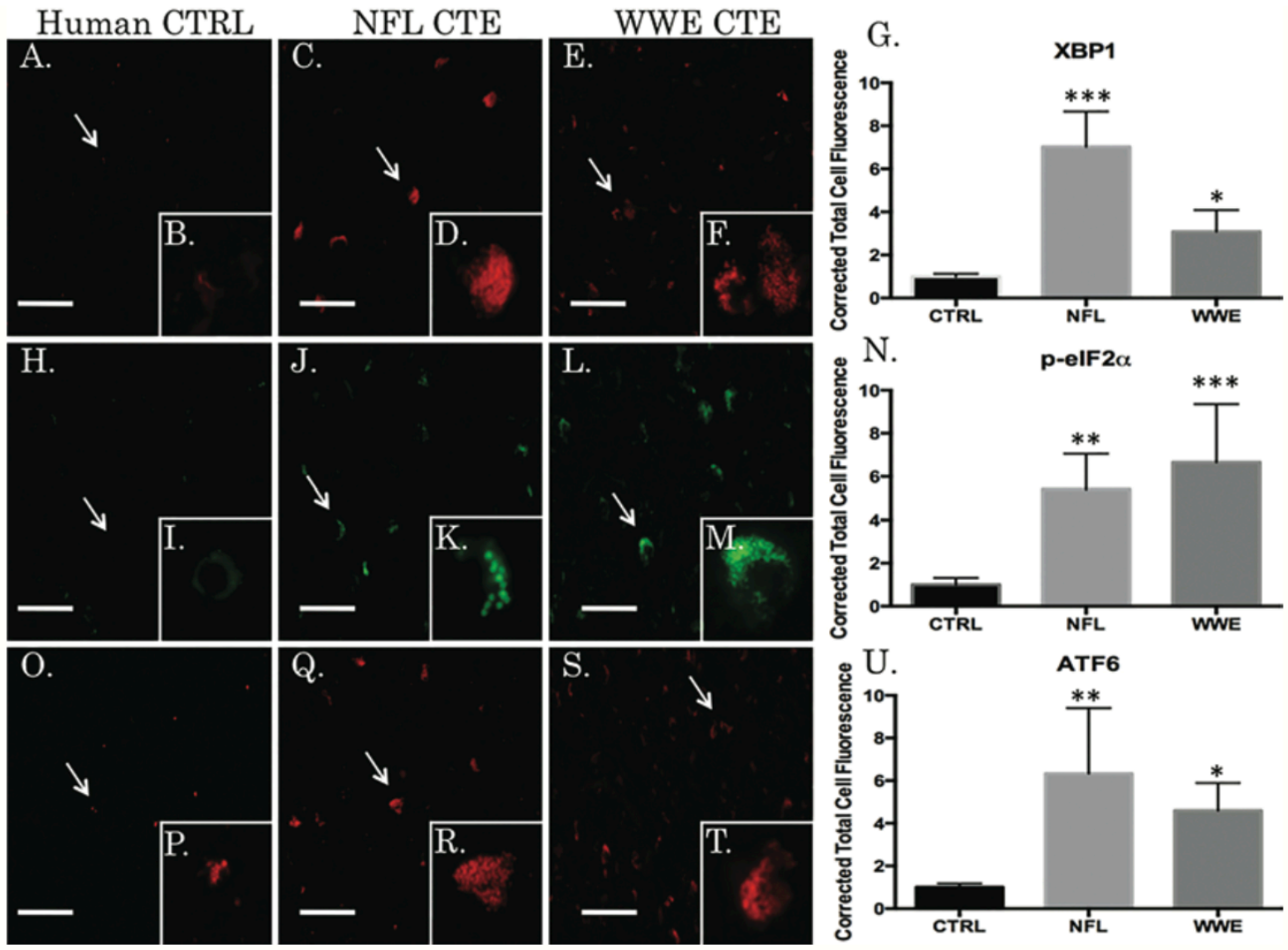

Figure 5.4: Tauopathy implicated in the activation of the ER stress response. Since tau tangles are accumulations of aggregated protein, the cell recognizes an intracellular disturbance that triggers the release of calcium. The calcium rush is directly responsible for ER stress activation as well as initiating the ER stress response. All three arms of the ER stress cascade were increased in human CTE samples. XBP1, arm 2 of the ER stress response, was significantly elevated in the NFL CTE brain $(P<0.001)(\mathbf{C}) 20 \mathrm{x}$, scale bar $75 \mu \mathrm{m}$, and inlay (D) 63x and in the WWE CTE brain $(P<0.05)(\mathbf{E}) 20 \mathrm{x}$, scale bar $75 \mu \mathrm{m}$, and inlay (F) 63x compared with control (A) 20x, scale bar 75um, and inlay (B) $63 \mathrm{x}$ as depicted in the bar graph (G). P-eIF2 $\alpha$, arm 1 of the ER stress response, was significantly elevated in the NFL CTE brain $(P<0.01)(\mathbf{J}) 20 \mathrm{x}$, scale bar $75 \mu \mathrm{m}$, and inlay $(\mathbf{K}) 63 \mathrm{x}$ and in the WWE CTE brain $(P<0.001)(\mathbf{L}) 20 \mathrm{x}$, scale bar $75 \mu \mathrm{m}$, and inlay $(\mathbf{M}) 63 \mathrm{x}$ 


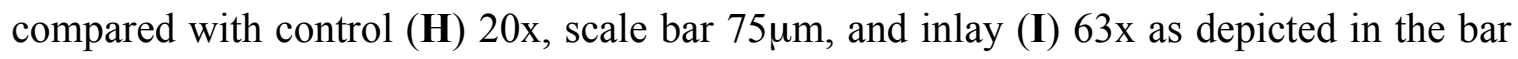
graph (N). ATF6, arm 3 of the ER stress response, was significantly elevated in NFL CTE brain $(P<0.01)(\mathbf{Q}) 20 x$, scale bar $75 \mu \mathrm{m}$, and inlay $(\mathbf{R})$ 63x and in the WWE CTE brain $(P<0.05)(\mathbf{S}) 20 x$, scale bar $75 \mu \mathrm{m}$, and inlay (T) 63x compared with control $(\mathbf{O})$ 20x, scale bar $75 \mu \mathrm{m}$, and inlay (P) $63 \mathrm{x}$ as depicted in the bar graph $(\mathbf{U})$. 

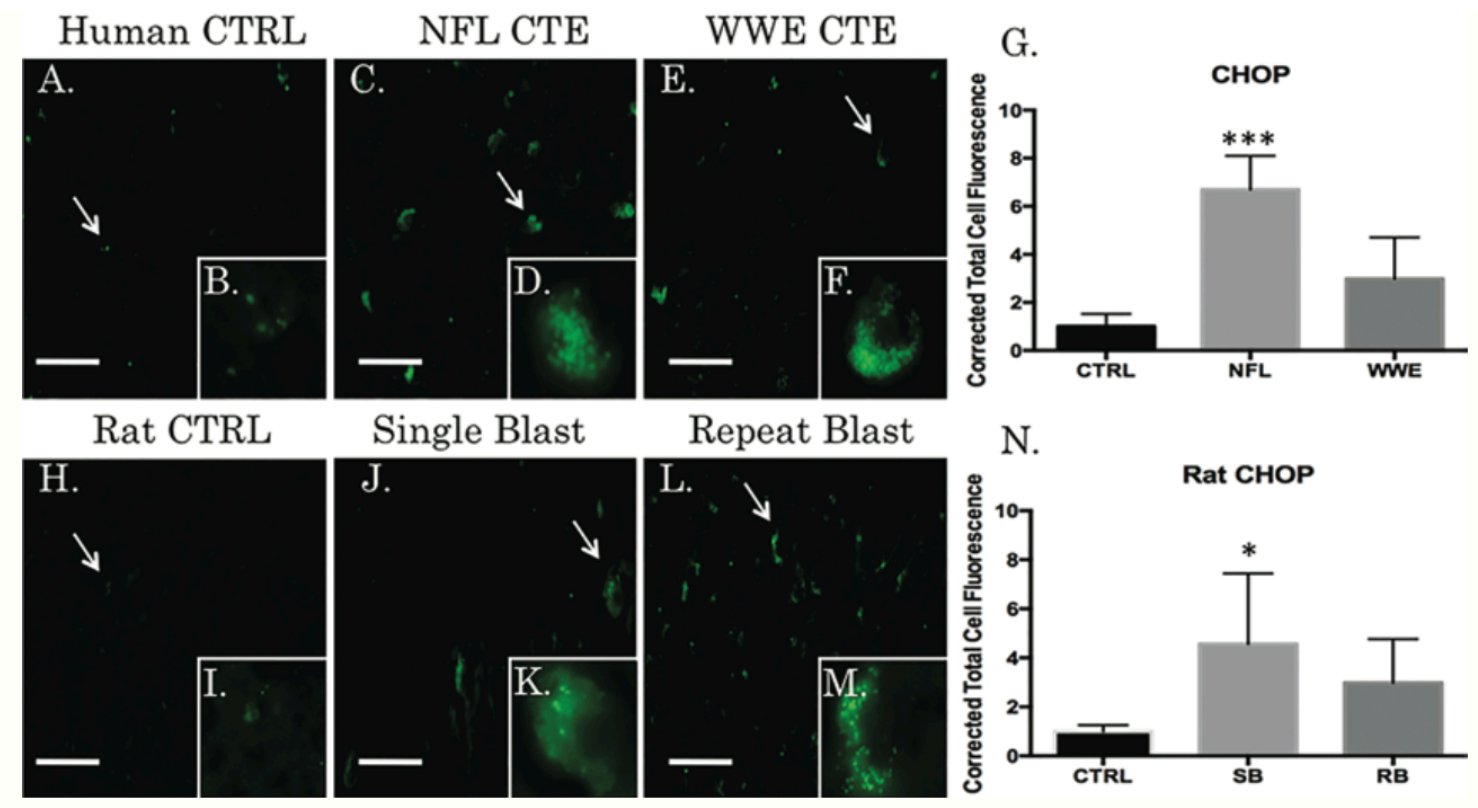

Figure 5.5: CHOP: an important component of ER stress. A baseline level of CHOP is necessary in order for a cell to return to homeostasis. If CHOP is pushed above threshold, apoptosis and tau hyperphosphorylation can occur. CHOP was significantly elevated in the NFL CTE brain $(P<0.001)($ C) $20 x$, scale bar $75 \mu \mathrm{m}$, and inlay (D) $63 \mathrm{x}$ but not in the

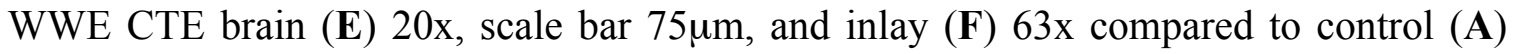
20x, scale bar $75 \mu \mathrm{m}$, and inlay (B) $63 \mathrm{x}$ as depicted in the bar graph (G). CHOP was also significantly elevated in single blast exposed animals $(P<0.05)(\mathbf{J}) 20 \mathrm{x}$, scale bar $75 \mu \mathrm{m}$, and inlay (K) 63x but not in repeat blast exposed animals (L) 20x, scale bar $75 \mu \mathrm{m}$, and inlay (M) 63x compared to control (H) 20x, scale bar 75 $\mu$ m, and inlay (I) 63x as depicted in the bar graph $(\mathbf{N})$. 


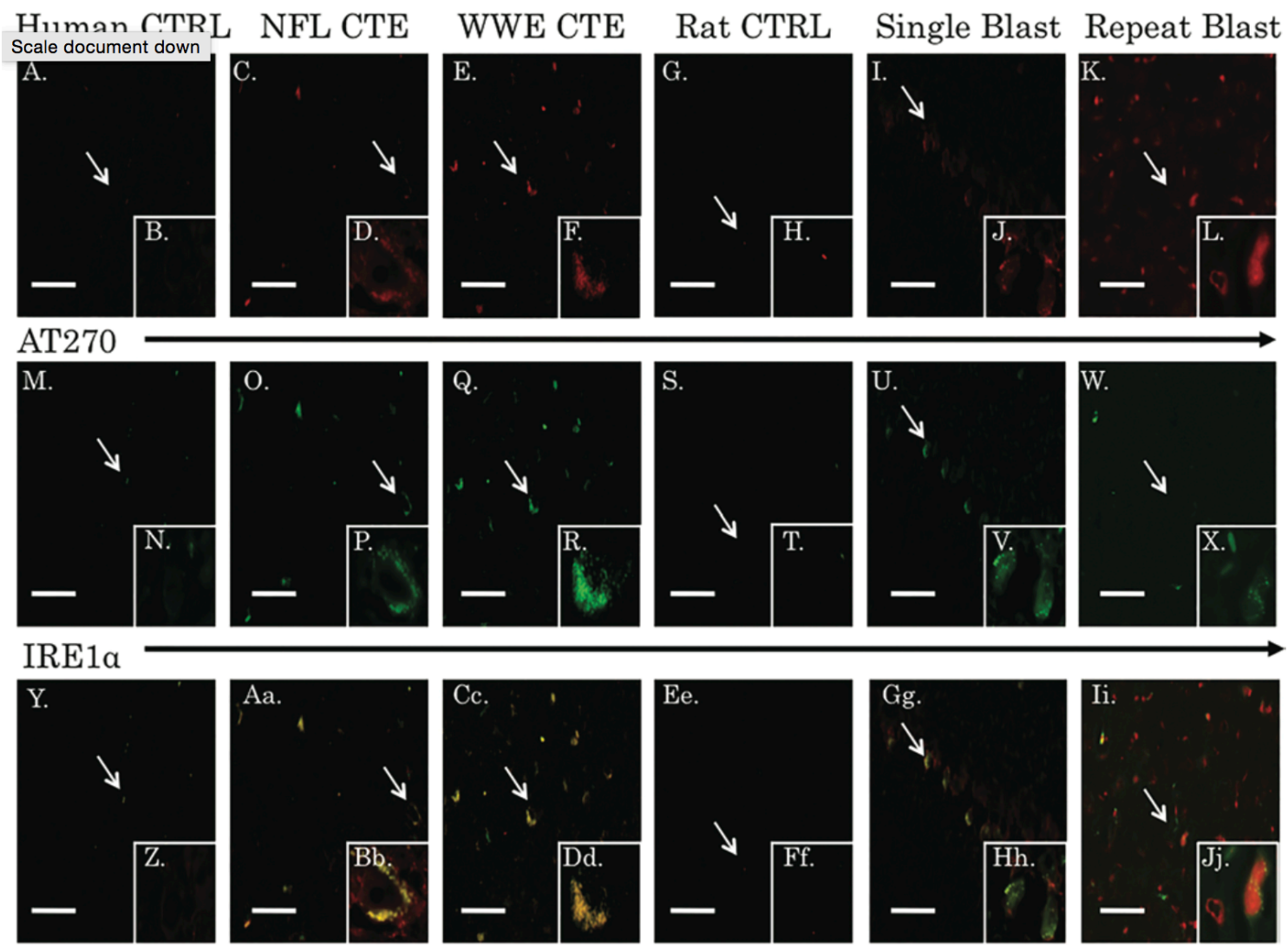

Overlay

Figure 5.6: AT270 co-localized with IRE1 $\alpha$. AT270 is a marker for tau hyperphosphorylation at threonine 181. Tau hyperphosphorylation occurs after tau has dissociated from tubulin and transported from the axon to the soma. What is unknown is if ER stress occurs in the same cells where tau hyperphosphorylation is present. We show that IRE1 $\alpha$, marker of arm 2 of the ER stress response, has a strong overlap coefficient with AT270 for NFL CTE brain $(r=0.846)$ and WWE brain $(r=0.904)$ compared to weak Pearson's coefficient for control $(r=0.286)$. Control has minimal AT270 (red) (A) 20x, scale bar $75 \mu \mathrm{m}$, and inlay (B) 63x, IRE1 $\alpha$ (green) (M) 20x, scale bar $75 \mu \mathrm{m}$, and inlay (N) 63x, and overlap (yellow) (Y) 20x, scale bar 75um, and inlay (Z) 63x. NFL CTE brain has apparent AT270 (red) (C) 20x, scale bar 75 4 m, and inlay (D) 63x, IRE1 $\alpha$ 
(green) (O) 20x, scale bar 75 $\mathrm{m}$, and inlay (P) 63x, and overlap (yellow) (Aa) 20x, scale bar 75um, and inlay (Bb) 63x. WWE CTE brain has apparent AT270 (red) (E) 20x, scale

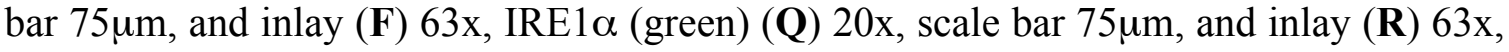
and overlap (yellow) (Cc) 20x, scale bar $75 \mu \mathrm{m}$, and inlay (Dd) 63x. IRE1 $\alpha$ also has strong overlap coefficient with AT270 for single blast exposed animals $(r=0.934)$ and repeat blast exposed animals $(r=0.807)$ compared to weak Pearson's coefficient for control $(\mathrm{r}=0.374)$. Control has minimal AT270 (red) $(\mathbf{G}) 20 \mathrm{x}$, scale bar $75 \mu \mathrm{m}$, and inlay (H) 63x, IRE1 $\alpha$ (green) (S) 20x, scale bar 75 $\mu$, and inlay (T) 63x, and overlap (yellow) (Ee) 20x, scale bar 75um, and inlay (Ff) 63x. Single blast exposed animal has apparent AT270 (red) (I) 20x, scale bar 75 $\mu$ m, and inlay (J) 63x, IRE1 $\alpha$ (green) (U) 20x, scale bar $75 \mu \mathrm{m}$, and inlay (V) 63x, and overlap (yellow) $(\mathbf{G g}) 20 \mathrm{x}$, scale bar $75 \mu \mathrm{m}$, and inlay (Hh) 63x. Repeat blast exposed animal has apparent AT270 (red) (K) 20x, scale bar 75um, and inlay (L) 63x, IRE1 $\alpha$ (green) (W) 20x, scale bar 75 $\mu$ m, and inlay (X) 63x, and overlap

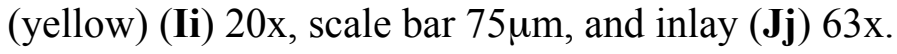


Human CTRL NFL CTE WWE CTE Rat CTRL Single Blast Repeat Blast

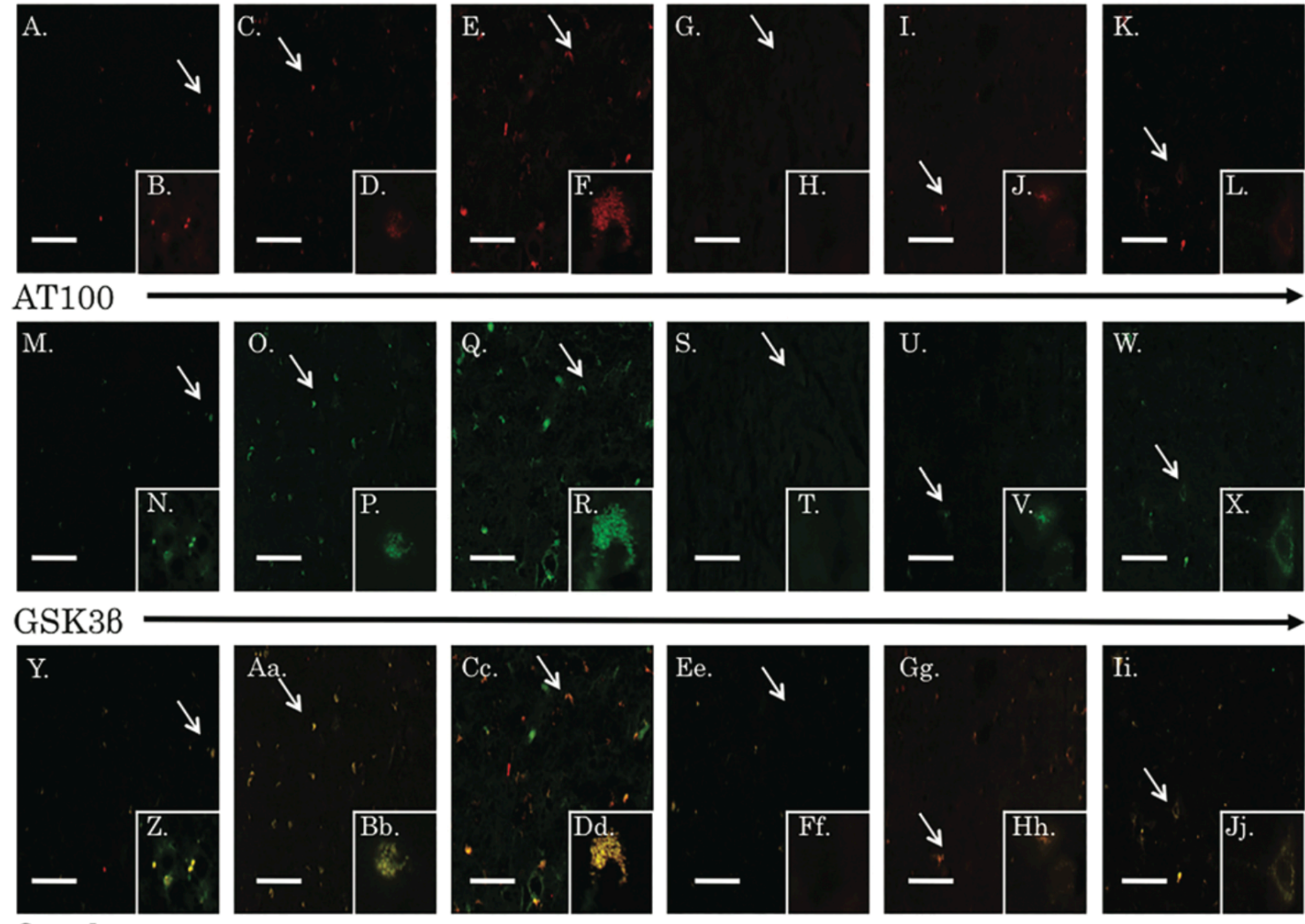

Overlay

Figure 5.7: AT100 is a marker of tau hyperphosphorylation at threonine 212 and serine 214. Tau is phosphorylated after injury by a select few kinases. GSK3 $\beta$ is by far the most important of these kinases accounting for over $1 / 3$ of all tau hyperphosphorylation. We show that GSK3 $\beta$ has a strong overlap coefficient with AT100 for NFL CTE brain $(r=$ $0.881)$ and WWE brain $(\mathrm{r}=0.881)$ compared to weak Pearson's coefficient for control (r $=0.173$ ). Control has minimal AT100 (red) (A) 20x, scale bar 75um, and inlay (B) 63x, GSK3 $\beta$ (green) (M) 20x, scale bar 75 $\mathrm{m}$, and inlay (N) 63x, and overlap (yellow) (Y) 20x, scale bar 75um, and inlay (Z) 63x. NFL CTE brain has apparent AT100 (red) (C) 20x, scale bar $75 \mu \mathrm{m}$, and inlay (D) 63x, GSK3 $\beta$ (green) (O) 20x, scale bar $75 \mu \mathrm{m}$, and inlay (P) 63x, and overlap (yellow) (Aa) 20x, scale bar 75um, and inlay (Bb) 63x. WWE 
CTE brain has apparent AT100 (red) (E) 20x, scale bar 75 $\mu$ m, and inlay (F) 63x, GSK3 $\beta$ (green) (Q) 20x, scale bar 75 $\mu$ m, and inlay (R) 63x, and overlap (yellow) (Cc) 20x, scale bar $75 \mu \mathrm{m}$, and inlay (Dd) $63 \mathrm{x}$. GSK3 $\beta$ also has strong overlap coefficient with AT100 for single blast exposed animals $(r=0.931)$ and repeat blast exposed animals $(r=0.887)$ compared to weak Pearson's coefficient for control $(r=0.068)$. Control has minimal AT100 (red) (G) 20x, scale bar 75 $\mu$, and inlay (H) 63x, GSK3 $\beta$ (green) (S) 20x, scale bar $75 \mu \mathrm{m}$, and inlay (T) 63x, and overlap (yellow) (Ee) 20x, scale bar $75 \mu \mathrm{m}$, and inlay (Ff) 63x. Single blast exposed animal has apparent AT100 (red) (I) 20x, scale bar 75um, and inlay (J) 63x, GSK3 $\beta$ (green) (U) 20x, scale bar 75um, and inlay (V) 63x, and overlap (yellow) (Gg) 20x, scale bar 75 $\mu$ m, and inlay (Hh) 63x. Repeat blast exposed animal has apparent AT100 (red) (K) 20x, scale bar 75 $\mu$ m, and inlay (L) 63x, GSK3 $\beta$ (green) (W) 20x, scale bar 75 $\mathrm{m}$, and inlay (X) 63x, and overlap (yellow) (Ii) 20x, scale bar $75 \mu \mathrm{m}$, and inlay $(\mathbf{J j}) 63 \mathrm{x}$. 


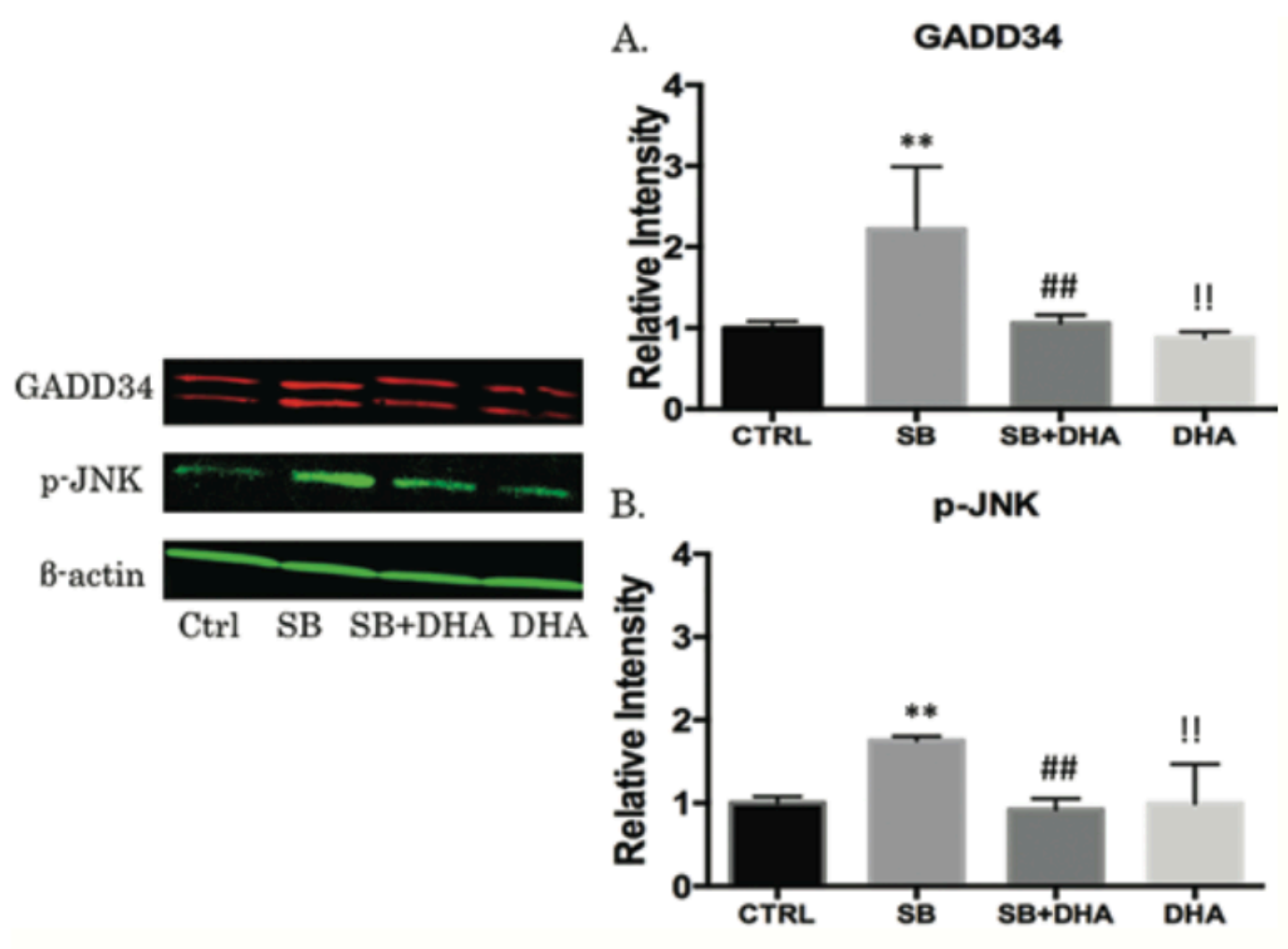

Figure 5.8: DHA reduces acute ER stress markers. Arms 1 and 2 of the unfolded protein response are increased acutely after injury. GADD34 is a downstream marker of the first arm of the ER stress response and p-JNK is downstream of the second arm. A significant difference between groups for the acute markers was observed 24 hours after blast exposure. (A) The single blast group had increased expression of GADD34 compared to control (** $P<0.01$ ), compared to DHA + single blast group (\#\# $P<0.01$ ), and compared to DHA alone group $(! ! P<0.01)$. (B) The single blast group had increased phosphorylation of JNK compared to control $(* * P<0.01)$, compared to DHA + single blast group (\#\# $P<0.01$ ), and compared to DHA alone group $($ !! $P<0.01)$. 


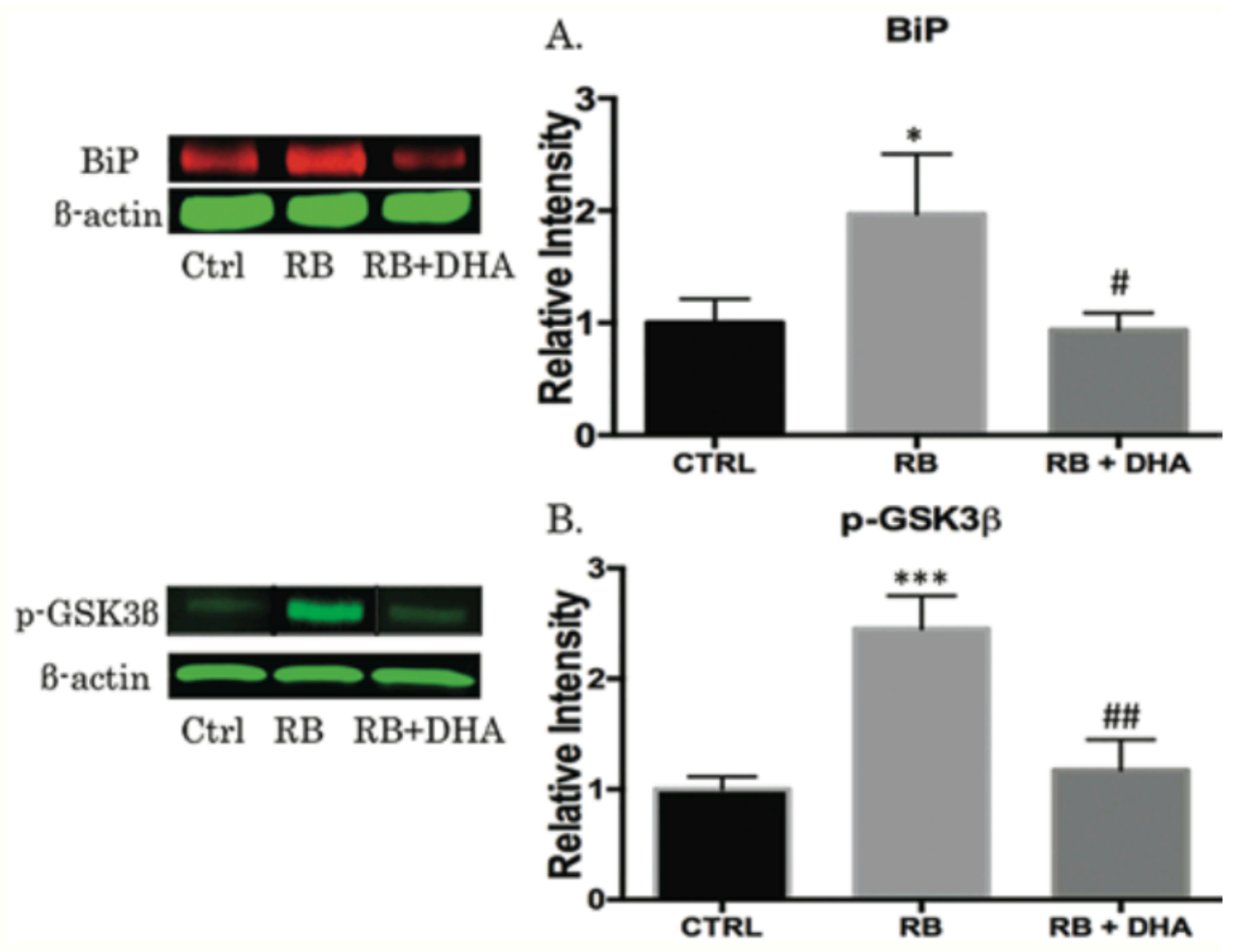

Figure 5.9: ER stress plays an important role in activation of tau kinases. Tau hyperphosphorylation feeds back and activates the ER stress response by causing the dissociation of BiP from the ER membrane. A significant difference between groups was observed 3 weeks post repetitive blast exposure for BiP. (A) The repeat blast group had increased levels of BiP compared to control $(* P<0.05)$, and compared to the repeat blast + DHA group (\# $P<0.05$ ). Activation of the tau kinase GSK3 $\beta$ can lead to increased tau pathology following TBI. A significant difference between groups was reported for p-GSK3 $\beta$. (B) The repeat blast group has increased phosphorylation of GSK3 $\beta$ at tyrosine 216 compared to control $(* * * P<0.001)$, and compared to the repeat blast + DHA group (\#\# $P<0.01)$. 
A.
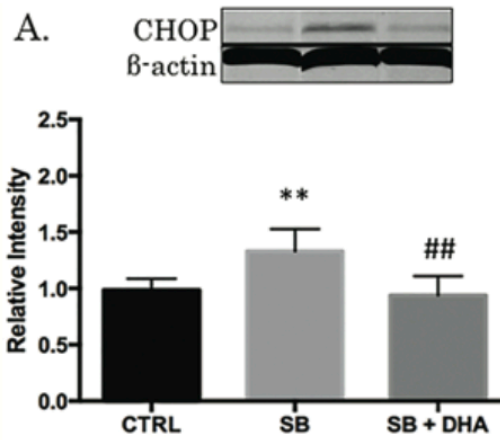

B.
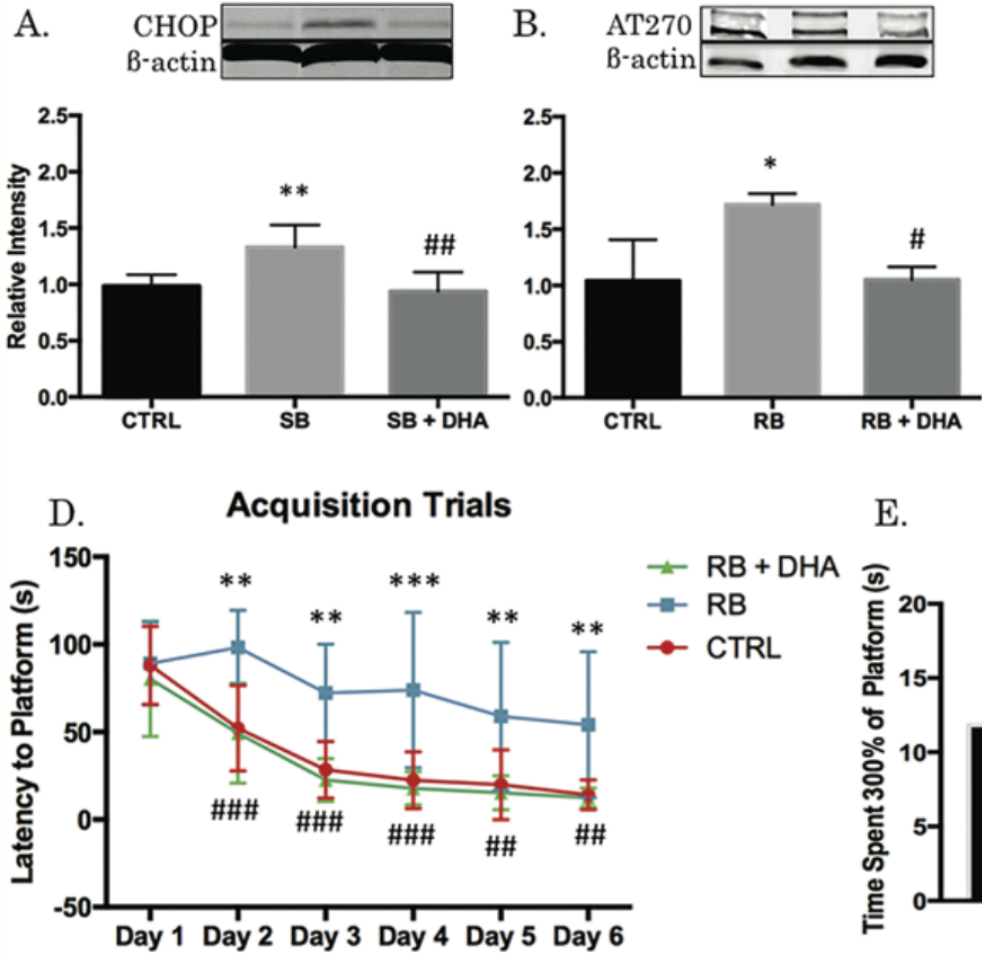
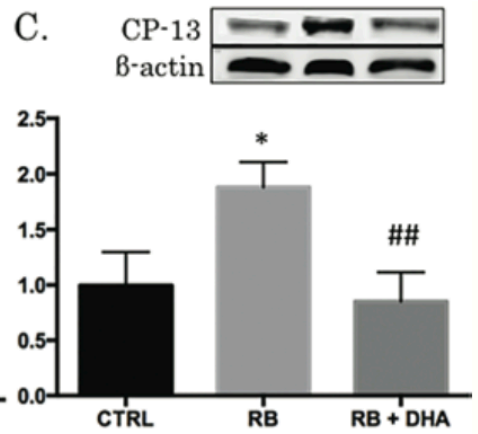

E.
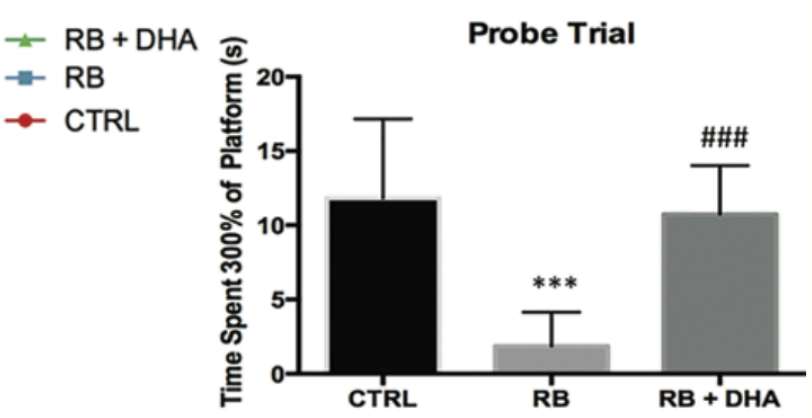

Figure 5.10: DHA decreases ER Stress and tau while preventing cognitive decline. CHOP is a key downstream target of all three arms of the ER stress pathway. Among its many roles, $\mathrm{CHOP}$ contributes to apoptosis, activation of tau kinases, and disruption of the mitochondria. A significant difference between groups was observed 24 hours after blast exposure. (A) The single blast group had a significant increase in $\mathrm{CHOP}(P<0.01)$ compared to control. The DHA + blast group had a significant reduction in $\mathrm{CHOP}(P<$ 0.01) compared to the single blast group only. AT270 is a marker of tau hyperphosphorylation that symbolizes the transition phase for tauopathy development. (B) Repeat blast increased AT270 compared with control $(P<0.05)$. DHA + repeat blast significantly reduced AT270 $(P<0.05)$ compared with repeat blast only. $(\mathbf{C})$ Repeat blast increased CP-13 compared with control $(P<0.05)$. DHA + repeat blast significantly reduced CP-13 $(P<0.05)$ compared with repeat blast only. Morris water maze is a test of 
cognitive performance and the ability to learn. The protocol used for this experiment consisted of 6 learning trial days followed by a probe trial on day 7. For each learning trial session, the animal was placed into the maze at 4 different start positions. Latency to platform was measured from each start position for up to 2 minutes. If the animal did not reach the platform in 2 minutes, they were placed on it for 15 seconds. On probe trial day the platform was removed and the animal was put into the maze at a novel start position and allowed to swim for 1 minute. Time spent in an area $300 \%$ of where the platform had been was recorded. (D) A significant difference in learning across trial days $(P<0.001)$ and between groups $(P<0.001)$ was observed. A significant difference between control and repeat blast groups for latency to platform was observed on day $2(\mathrm{t}=3.633, P<$ $0.01)$, day $3(\mathrm{t}=3.455, P<0.01)$, day $4(\mathrm{t}=4.057, P<0.001)$, day $5(\mathrm{t}=3.079, P<$ $0.01)$, and day $6(\mathrm{t}=3.149, P<0.01)$. A significant difference in latency to platform was also seen between repeat blast and repeat blast + DHA groups on day $2(\mathrm{t}=3.855, P<$ $0.001)$, day $3(\mathrm{t}=3.918, P<0.001)$, day $4(\mathrm{t}=4.415, P<0.001)$, day $5(\mathrm{t}=3.442, P<$ $0.01)$, and day $6(\mathrm{t}=3.283, P<0.01)$. (E) A significant difference between control and repeat blast groups $(P<0.001)$ and between repeat blast and repeat blast + DHA groups $(P<0.001)$ was seen on probe trial. 


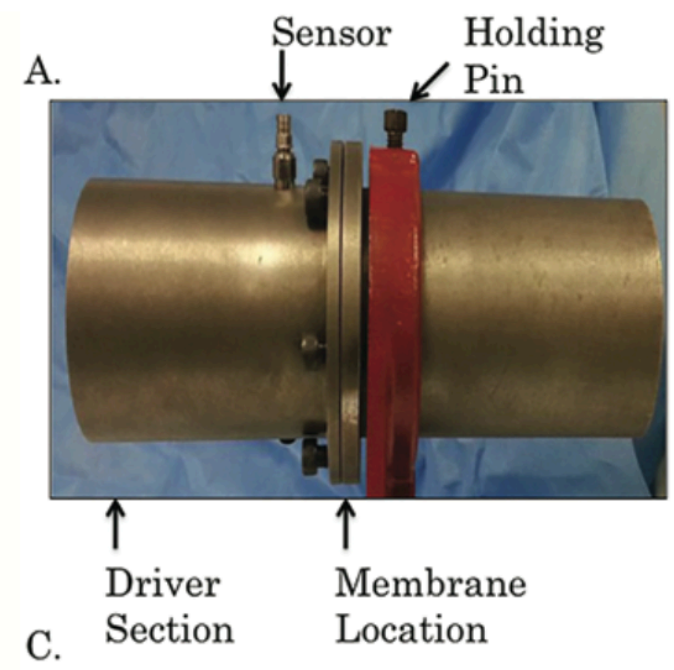

B.
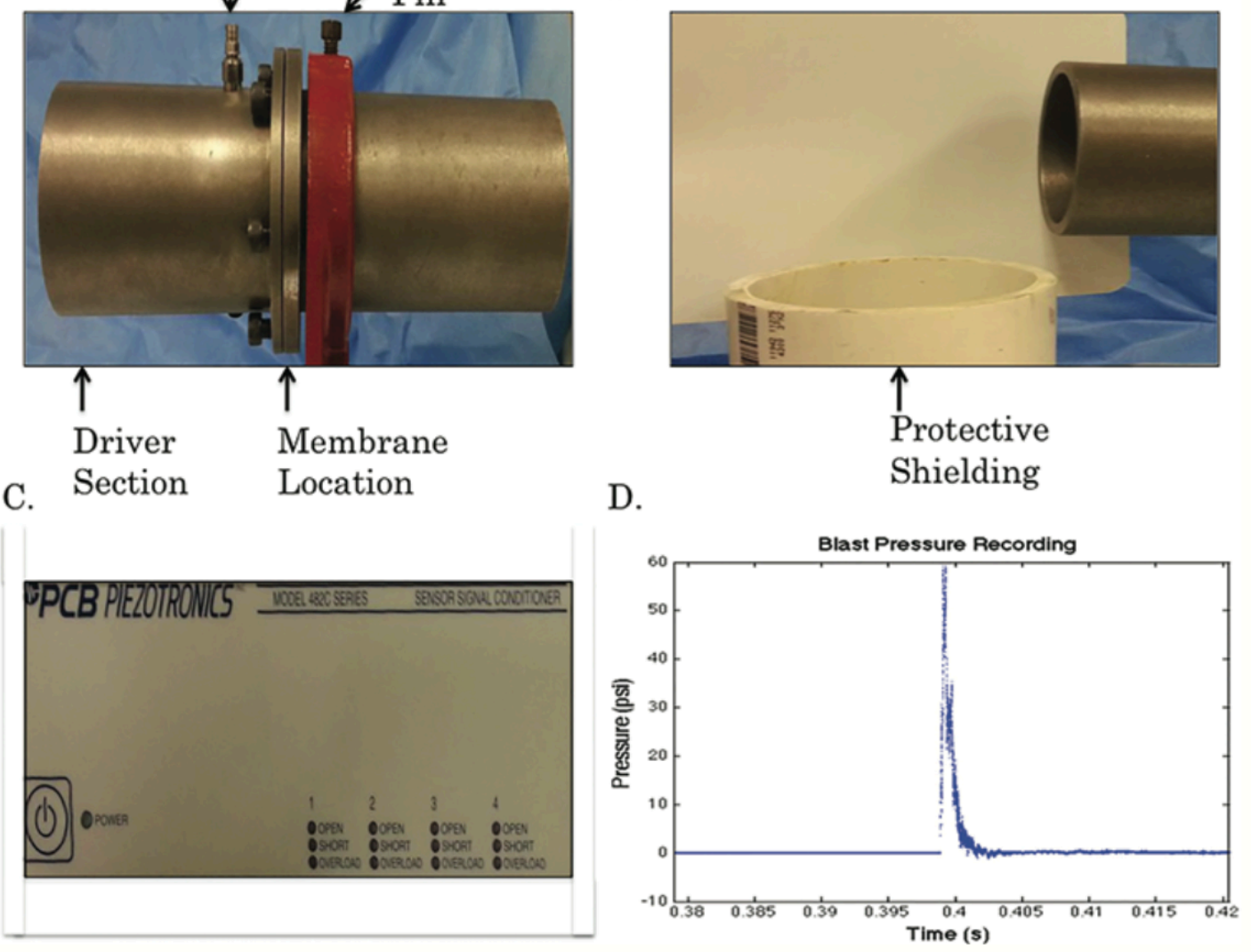

Supplementary Figure 5.1: Representative set-up of blast model. (A) blast model with piezoelectric sensor and driver section. (B) polyvinyl tube that protects the animal's body from blast wave. (C) sensor signal conditioner. (D) graph showing peak overpressure recorded from $\sim 50$ PSI blast wave. 
A.

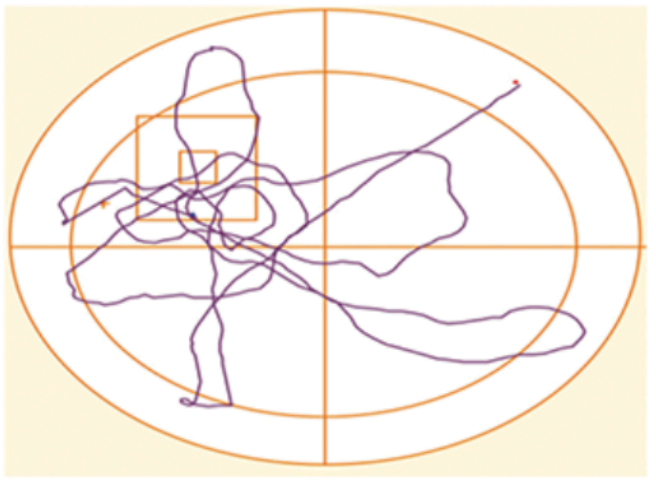

C.

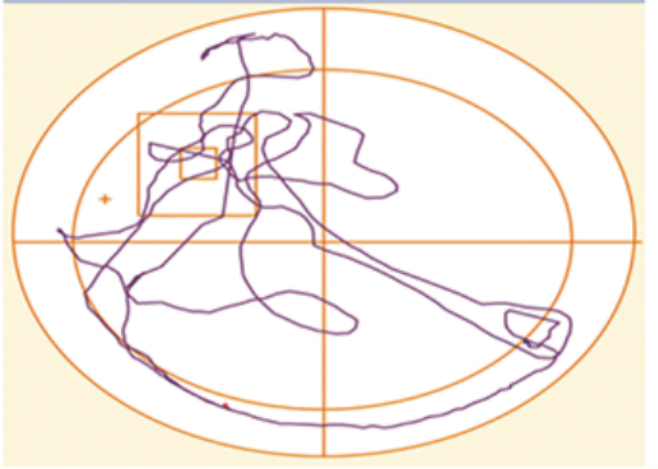

B.

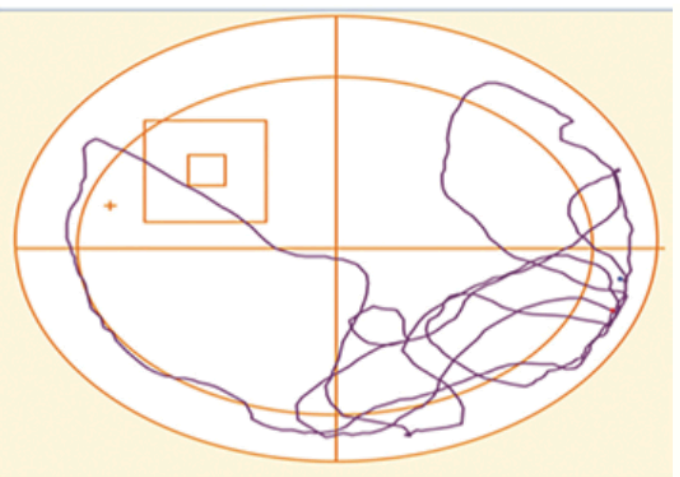

Supplementary Figure 5.2: Representative track plots for Morris water maze on probe trial day. (A) track plot for control animal. (B) track plot for repeat blast animal. (C) track plot for repeat blast + DHA animal. 


\section{Chapter Six}

Amelioration of NADPH-mediated stress reduces cell death following blast-induced traumatic brain injury

This work is published in Transl Res. 2015 Dec;166(6):509-528.e1. doi:

10.1016/j.trs1.2015.08.005. Epub 2015 Sep 8. PMID: 26414010

Lucke-Wold BP, Naser ZJ, Logsdon AF, Turner RC, Smith KE, Robson MJ, Bailes JE,

Lee JM, Rosen CL, Huber JD 


\section{ABSTRACT}

1.7 million traumatic brain injuries (TBIs) occur each year in the United States, but available pharmacologic options for the treatment of acute neurotrauma are limited. Oxidative stress is an important secondary mechanism of injury that can lead to neuronal apoptosis and subsequent behavioral changes. Utilizing a clinically relevant and validated rodent blast model, we investigated how NADPH oxidase expression and associated oxidative stress contributes to cellular apoptosis following single and repeat blast injuries. Nox4 forms a complex with p22phox following injury, forming free radicals at neuronal membranes. Using immunohistochemical-staining methods, we found a visible increase in Nox4 following single blast injury in Sprague Dawley rats. Interestingly, Nox4 was also increased in post-mortem human samples obtained from athletes diagnosed with chronic traumatic encephalopathy (CTE). Nox4 activity correlated with an increase in superoxide formation. Alpha lipoic acid, an oxidative stress inhibitor, prevented the development of superoxide acutely, and increased anti-apoptotic markers Bcl-2 ( $\mathrm{t}=$ $3.079, \mathrm{p}<0.05)$ and heme oxygenase $1(\mathrm{t}=8.169, \mathrm{p}<0.001)$ after single blast. Subacutely, alpha lipoic acid treatment reduced pro-apoptotic markers $\operatorname{Bax}(t=4.483, p<0.05)$, caspase $12(\mathrm{t}=6.157, \mathrm{p}<0.001)$, and caspase $3(\mathrm{t}=4.573, \mathrm{p}<0.01)$ following repetitive blast, and reduced tau hyperphosphorylation indicated by decreased CP-13 and PHF staining. Alpha lipoic acid ameliorated impulsive-like behavior 7 days after repetitive blast injury $(t=3.573, p<0.05)$ compared to blast exposed animals without treatment. TBI can cause debilitating symptoms, and psychiatric disorders. Oxidative stress is an ideal targets for neuropharmacologic intervention, and alpha lipoic acid warrants further investigation as a therapeutic for prevention of chronic neurodegeneration. 
KEY WORDS: Blast traumatic brain injury, NADPH oxidative stress, lipoic acid, apoptosis, impulsive-like behavior

\section{INTRODUCTION}

Traumatic brain injury (TBI) remains an immense public health burden in the United States, and continues to be a leading cause of morbidity and mortality in individuals under the age of 45-years-old (Bruns \& Hauser 2003). Mild TBI can lead to progressive motor, cognitive, and behavioral decline (Bramlett \& Dietrich 2014). These long-term outcomes result in enormous societal costs (Maas et al 2008). In particular, blast-induced TBI has been classified as the 'signature injury' of the recent wars in Iraq and Afghanistan. National and international emphasis has been placed on how acute neurotrauma leads to chronic neurodegeneration over time. The progression depends on both severity and number of exposures. In order to investigate the pathologic process that contributes to neurodegeneration, scaled injury in animal models must be ideally utilized. A blast wave injury has three primary effects: wave transport through tissue, acceleration/deceleration causing axonal shearing, and disruption of the blood brain barrier (BBB). Typically, the primary damage of TBI is followed by secondary injury, which can develop at a later date. The initial insult can lead to major brain damage and loss of functional outcome. Secondary injury, on the other hand, triggers a cascade of biochemical events leading to neuroinflammation, brain edema, and delayed neuronal cell death and neurodegeneration (Zhang et al 2012). 
Delayed neuronal cell death is carefully mediated through the process of apoptosis. When the cell is damaged beyond repair, a signaling cascade is initiated, which can lead to cell destruction. Many pathways contribute to apoptosis activation in the cell, but one of the main methods is the development and propagation of free radical oxygen species (ROS). The creation of ROS has been linked to the activation of NADPH oxidase. In a non-injury state, NADPH oxidase participates in cellular host defense through cytokine signaling, gene expression regulation, posttranslational processing of protein, endoplasmic reticulum stress response, and tissue homeostasis (Gao et al 2012). Only after injury or infection does the NADPH oxidase pathway contribute to ROS formation. Inhibiting the NADPH oxidase, Nox2, with apocynin following TBI has provided beneficial behavioral and histopathologic results (Loane et al 2013, Lu et al 2014). Lipoic acid is another readily available supplement that can inhibit the formation of ROS, but does not cross mitochondrial membranes thereby targeting plasma membrane NADPH activity (Gomes \& Negrato 2014). Although the mechanism is not fully known, alpha lipoic acid can reduce toxic free radicals and decrease NADPH oxidase activity (Park et al 2014). We sought to investigate the effect of lipoic acid on the less well-studied NADPH oxidase pathway, Nox4, which is found at neuronal plasma membranes (Supplementary Fig. 1).

The Nox4 pathway can lead to the generation of the superoxide radical $\left(\mathrm{O}_{2}{ }^{-}\right)$ (Zhang et al 2012). The superoxide radical is produced when an oxygen molecule gains a single electron from another substance. Excess $\mathrm{O}_{2}{ }^{-}$can form the toxic hydroxyl radical $\left(\mathrm{OH}^{-}\right)$through interaction with hydrogen peroxide $\left(\mathrm{H}_{2} \mathrm{O}_{2}\right)$. The hydroxyl radical can eventually combine with nitric oxide to form peroxynitrite $\left(\mathrm{ONO}_{2}{ }^{-}\right)$(Zhang et al 2012). 
Peroxynitrite has devastating effects on cellular membranes. Historically, mitochondria dysfunction has been postulated as the primary source of free radical species generation, but recent evidence has shown that NADPH oxidase can also contribute to reactive species development. NADPH oxidase is a composite membrane enzyme made up of Nox and phox subunits that associate with a series of supporting proteins (Bedard \& Krause 2007, Sorce \& Krause 2009). The Nox subunits come in one of five isoforms, and each isoform is heavily concentrated in a specific organ. Nox4 is one of the isoforms most heavily concentrated within the central nervous system. Nox4 is constitutively active and produces large amount of superoxide following injury. Through interaction with cellular components, Nox4 generates hydrogen peroxide and subsequently the potent hydroxyl radical (Gao et al 2012). Nox4 has not been previously studied for its role in ROS formation following blast injury, therefore opening an important avenue for investigating a novel and potentially important therapeutic target.

Oxidative stress induction and the consequential damage that occurs as a result of blast exposure is likely profound, but the full extent of injury has yet to be elucidated. In this work, we investigate the effects of NADPH oxidase, specifically Nox4 activity, in the progression towards apoptosis and tau hyperphosphorylation following a moderate blast injury 24 hours after blast exposure and 2 weeks after repetitive blast exposure. We selectively targeted the Nox 4 response at the neuronal plasma membrane using the antioxidant alpha-lipoic acid. Alpha lipoic acid is a natural occurring compound found in multiple cell types that acts as a co-factor in oxidative metabolism reactions (Wei et al 2015). It is thioctic acid with a disulfide bond, chirality, and a dithialone ring (Reljanovic et al 1999). The bioavailability is $30 \%$ and it is metabolized to 5 metabolites by the liver, 
which are then cleared primarily by catabolism (Teichert et al 2005). It acts as a freeradical scavenger when administered peripherally and readily passes through the BBB and neuronal membranes but not mitochondrial membranes (Ozbal et al 2015). In particular, it has been shown to be a carbonyl scavenging compound following TBI (Hall et al 2010). Supplementation in humans following TBI has provided reduction in adverse symptoms (Curtis \& Epstein 2014). It reduces susceptibility for seizures as well as excitotoxicity (Meyerhoff et al 2004). We investigate its role in modulation of Nox4 for the prevention of the chronic sequelae associated with neurotrauma.

\section{MATERIALS AND METHODS}

The paper conforms to all relevant guidelines for human and animal research.

\section{Animals}

All procedures involving live animals were approved by the Institutional Animal Care and Use Committee of West Virginia University and were performed according to the principles of the Guide for the Care and Use of Laboratory Animals (NIH). This work used (40) forty $350 \mathrm{~g}$ male Sprague-Dawley rats acquired from Hilltop Lab Animals (Hilltop Lab Animals, Inc.). The method used to calculate the number of animals needed for a given study was determined using information from the book, Guidelines for the Care and Use of Mammals in Neuroscience and Behavioral Research. The number of animals was based on effect size, standard deviation, power, and significance. The equation to be used to calculate sample size is: $n=1+\left[2 C(s / d)^{2}\right]$, where $C$ is a value dependent upon the $a$ and $b$ values chosen. For our studies $a=0.05$ and $b=0.2$ for a power of 1-b=0.8. $\mathrm{C}_{(0.05,0.8)}=7.85$ (obtained from table). Effect size and standard deviation expected are variable per assay and were calculated based on numbers from previous 
work. Animals were acclimated for 1 week prior to experimental use and were housed under 12-hour light/12-hour dark conditions with food and water available ad libitum.

\section{Human brain sections}

Post-mortem formalin fixed brain sections were obtained from the entorhinal cortex of a retired professional football player and professional wrestler as well as an age and sex matched control. Descriptions of the chronic traumatic encephalopathy (CTE) cases have been previously described (Omalu et al 2010a, Omalu et al 2010b). Briefly, the professional wrestler committed suicide at age 40 . No atrophy or necrosis was observed in the brain. Neurofibrillary tau tangles were frequently scattered, and he had the apolipoprotein E3/E3 allele. The professional football player died from traumatic injury at age 36 . The brain was grossly intact with widely spread tauopathy. No other neurodegenerative disease diagnostic features were found in either case. The age and sex matched control did not have a known history of neurotrauma and no evidence of tau pathology.

\section{Blast exposure}

Rats were exposed to traumatic brain injury via a moderate blast wave exposure characterized previously by our lab (Turner et al 2013). Briefly, the animals were anesthetized with $4 \%$ isoflurane prior to blast exposure. Animals were then oriented with the long axis of the animal perpendicular to the blast front. The blast was delivered perpendicular to the head with the wave encountering the right side of the head (directly) before passing through the skull and exiting the left side (indirectly). The thorax and abdomen were protected using rigid shielding. The design allows for acceleration and 
deceleration to occur freely because the head is not fixed, similar to battlefield exposure. Animals were divided into the following groups for the 24 hour study: control $(n=4)$, lipoic acid only $(n=4)$, moderate blast exposure only $(n=4)$, and moderate blast exposure along with lipoic acid treatment $(\mathrm{n}=4)$. Immediately following blast, the animals were returned to a holding cage with a homeothermic heating blanket with rectal thermometer to maintain body temperature at $37^{\circ} \mathrm{C}$. Once reflexes were restored, animals were returned to the home cage.

For repetitive blast exposure, animals received blast every other day for a total of six blast exposures over a two-week period. The groups used for this study were control $(\mathrm{n}=8)$, repeat blast $(\mathrm{n}=8)$, and repeat blast along with lipoic acid treatment $(\mathrm{n}=8)$. The animals were exposed to the same intensity blast wave as the animals from the 24-hour experimental group. Animals were returned to a holding cage for monitoring before being placed in their home cage. Animals were anesthetized with $4 \%$ isoflurane before every blast exposure. Animals were sacrificed 2 weeks after final blast exposure.

\section{Alpha lipoic acid treatment(s)}

Alpha lipoic acid was obtained from Sigma Aldrich. It was dissolved in sterile saline solution with $10 \%$ ethanol $(0.1 \mathrm{~mL} / 10 \mathrm{~g}$ body weight) (Teknova, Fisher Scientific, Pittsburgh, PA). For animals that were part of the drug treatment group, the animals were administered $10 \mathrm{mg} / \mathrm{kg}$ alpha lipoic acid 5 minutes after each blast exposure via i.p. injection. Animals receiving blast injury only or controls received an i.p. injection of saline after each blast. Animals were closely monitored and upon full recovery were returned to the home cage.

\section{Elevated plus maze}


The EPM was elevated $60 \mathrm{~cm}$ from the floor. The two open arms intersected perpendicular to the two closed arms. Each arm was $50 \mathrm{~cm}$ by $10 \mathrm{~cm}$. The closed arms were encased by black wood siding $30 \mathrm{~cm}$ tall on all exposed sides. Elevated plus maze assessment was conducted 7 days after repetitive blast exposure according to procedures outlined by Walf and Frye (Walf \& Frye 2007). The rats were placed in the center of the maze facing an open arm. Each animal was recorded for a total of 5 minutes with open access to every arm. ANY-Maze Version 4.63 video tracking software (Stoelting Co.) was used to record the number of entries into the open arm, time spent in the open arm, and distance traveled in the open arm. The ANY-Maze software settings were as follows: test duration 300 seconds and percentage of body to be considered in arm $90 \%$.

\section{Immunoblotting}

Protein was isolated from each respective sample by sonication in $500 \mu \mathrm{L}$ of hot $\left(85-95^{\circ} \mathrm{C}\right) 1 \%$ sodium docecyl sulfate (SDS) as previously described (O'Callaghan \& Sriram 2004). Samples were run using $50 \mu \mathrm{g}$ of protein/well using Tris-Glycine $10 \%$ selfpoured 10-well gels in combination with 5X Lammeli sample buffer. Gels were run using a mini-PROTEAN system (Bio-Rad) and transferred to polyvinylidene fluoride (PVDF) membranes (Bio-Rad) using wet electrophoretic transfer cells (Bio-Rad). Primary antibodies used were anti-Bcl-2 (rabbit) and anti-HO-1 (goat) at 1:200 (Santa Cruz Biotechnology, Santa Cruz, CA). An HRP-conjugated $\beta$-actin rabbit monoclonal antibody (Cell Signaling) was used as an endogenous control for all samples at a concentration of 1:10,000. Molecular weight determination was conducted using SeeBlue ${ }^{\circledR}$ Plus2 (Life Technologies, Carlsbad, CA). LI-COR secondary antibodies IRDye ${ }^{\circledR} 800 \mathrm{CW}$ (goat anti-rabbit) and IRDye ${ }^{\circledR} 680 \mathrm{RD}$ (goat anti-mouse) (LI-COR, 
Lincoln, NE) were used with an Odyssey fluorescent scanner at wavelengths 800 or 700 , intensity 6.0, and 84 resolution with high image quality. Images were converted to gray scale, analyzed after background subtraction, and normalized to $\beta$-actin to give relative overall intensity.

Samples used for carbonyl detection were isolated and sonicated in 6\% SDS.

Control samples were mixed with derivation control solution and treatment samples were mixed with $1 \mathrm{X}$ DNPH solution. The samples were incubated for 15 minutes followed by mixing with a neutralization solution. The samples were loaded $20 \mu \mathrm{l}$ per well and ran on a $10 \%$ gel as outlined above. The primary antibody and standards were from an OxyBlot Protein Oxidation Detection Kit (Millipore).

\section{Reactive oxygen species detection}

Brain tissue was harvested from each treatment group including control, single blast, and single blast with lipoic acid and prepared in accordance to procedures outlined in the ROS Detection kit manual. Briefly, tissue was homogenized and cells were isolated by incubation in collagenase at $2 \mathrm{mg} / \mathrm{ml}$ for approximately 30 minutes. Cells were then separated by enzyme digestion and manual disruption with repeated pipetting. Cells were strained through a 70nm nylon cell strainer followed by centrifugation at $400 \mathrm{xg}$ for 5 minutes. The pellet was then resuspended to a concentration of $0.5 \times 10^{6}$ $1.0 \times 10^{6}$ cells $/ \mathrm{ml}$ in Dulbecco's Modified Eagle Medium.

Total ROS and Superoxide were detected using a Total ROS/Superoxide Detection kit (Enzo Life Sciences) according to manufacturer's instructions for a fluorescent microplate assay. In brief, $100 \mu \mathrm{l}$ of suspended cells were added to each well of a dark walled 96 well plate with a clear bottom. The cells were incubated overnight at 
$37^{\circ} \mathrm{C}$ in Dulbecco's Modified Eagle Medium. Media was then removed the following day and $100 \mu \mathrm{l}$ of ROS/Superoxide Detection Solution, prepared using kit reagents, was added to each well and incubated in the dark for 60 minutes. Total concentrations of ROS determined by a glutathione based reaction (green) and Superoxide determined by a xanthine oxidase reaction (red/yellow) were detected at an excitation/emission of 488/520nm and 550/610nm respectively using a Biotek Synergy H1 Hybrid Reader. Data was collected using Gen5 2.01 software.

\section{Histological preparations}

Animals were anesthetized as described above and perfused transcardially with cold $0.9 \%$ saline followed by $10 \%$ formalin for a total of ten minutes. The brain was then extracted and placed into fresh $10 \%$ formalin for a minimum of $24 \mathrm{~h}$. After the fixation, the brain was blocked into sections and paraffin embedded as previously described (Turner et al 2012b). Briefly, tissues were processed using the Tissue-Tek Q4 170 VIP 5 automatic tissue process (Sakura Finetek) and embedded in paraffin using the Tissue-Tek TEC 5 embedding system (Sakura Finetek). Tissues were sliced using a Leica RM2235 microtome (Leica Microsystems) and slices mounted on slides for staining. Standard fluorescent and DAB staining protocols were utilized for staining for iron (Sigma Aldrich), 8-hydroxy-2-deoxyguanosine, Nox4, p22phox, and Bax (Santa Cruz), caspase 3 and caspase 12 (Cell Signaling), and PHF, MC1, and CP-13 (Kind gift from Dr. Peter Davies).

Images were acquired from the S1BF region of the cortex (10 slides per animal). Imaging was performed using a Zeiss Axio Imager 2 for brightfield images and Zeiss Axio Observer Z1 for fluorescent images shown. For fluorescent staining, 10 cells per 
slide were randomly selected, outlined, and measured with ImageJ software (NIH) by an observer blinded to experimental group. Density was adjusted per mean area to give corrected total cell fluorescence normalized to background. For DAB and iron staining, an observer blinded to experimental group selected 100 random cells per slide and recorded the ratio of positive cells to total cells.

\section{Data analysis}

Data was analyzed using GraphPad Prism 5.0 (GraphPad Software, La Jolla, CA). A one-way ANOVA with Tukey's post-hoc test for multiple comparisons was used for Western blot, fluorescent IHC, and elevated plus maze. Tukey's post-hoc multiple comparisons test is a pair wised comparison between each group with every other group. The graphs show the comparisons that were statistically significant. $\chi^{2}$ analyses were used for DAB and iron staining. A p-value $<0.05$ was considered statistically significant for all data analyzed.

\section{RESULTS}

\section{An increase is seen in intraparenchymal iron, Nox4 and p22phox after single blast}

Iron staining revealed an increase in intracellular iron deposition (19/100) 24 hours following blast injury in the left prefrontal cortex compared to control (2/100) (Figure 6.1 A). Free iron induces oxidative stress, which is toxic to neuronal plasma membranes (Uranga et al 2009). Lipoic acid administered 5 minutes post-blast leads to decreased intracellular accumulation of iron (11/100) compared to blast only animals with a $\chi^{2}=40.594, p<0.0001$. Nox 4 is found at plasma membranes and contributes to free radical formation (Zhang et al 2009). Nox4 was visibly increased (29/100) 24 hours following blast injury in the left prefrontal cortex compared to control (8/100) (Figure 6.1 
B). Lipoic acid visibly reduced Nox4 (12/100) when given 5 minutes post-blast with $\chi^{2}=29.079, p<0.0001$. P22phox forms an activation complex with Nox 4 to regulate downstream genetic markers. P22phox was also visibly increased (23/100) 24 hours after single blast in the left prefrontal cortex compared to control (6/100). Likewise, alpha lipoic acid treatment 5 minutes post-blast decreased p22phox (13/100) with $\chi^{2}=24.031$, $\mathrm{p}<0.0001$. Therefore, iron-mediated toxicity possibly acting through Nox4 oxidative stress is mitigated by alpha lipoic acid treatment.

\section{Nox4 activity increases superoxide formation but not total ROS after single blast}

The Nox4/P22phox complex can lead to the generation of superoxide. Superoxide can damage the cell if not readily cleared. Blast only animals had a visible increase in superoxide $24 \mathrm{~h}$ post-blast compared to control (Figure $6.2 \mathrm{~A}$ ). Lipoic acid administered 5 minutes post-blast visually decreased superoxide back to control levels. Fluorescent intensity measurement revealed a significant difference between groups for superoxide $(\mathrm{F}(2,15)=30.93, \mathrm{p}<0.001)$. Post-hoc comparison revealed that single blast exposure significantly increased superoxide compared to control $(q=9.1, p<0.001)$, but lipoic acid administered 5 minutes after single blast ameliorated this response $(q=10.09, p<0.001)$ (Figure 6.2 B). No significant difference was seen between groups for total $\operatorname{ROS}(F(2,15)$ $=3.568, \mathrm{p}=0.054)$ lending credence to a NADPH specific response verse mitochondria dependent response.

\section{Lipoic acid prevents formation of oxidative stress byproducts}

Previous studies have shown that byproducts of oxidative stress are significantly increased following TBI (Cheng et al 2013). In particular, 8-hydroxy-2-deoxyguanosine and carbonyls are elevated post-injury (Ferguson et al 2010). We found a significant 
difference between groups for 8-hydroxy-2-deoxyguanosine in the cortex 24 hours post blast $(\mathrm{F}(2,27)=28.18, \mathrm{p}<0.001)$ (Figure $6.3 \mathrm{~A})$. Tukey's post-hoc comparison showed a significant difference between control and blast $(\mathrm{q}=10.53, \mathrm{p}<0.001)$ and blast and blast + lipoic acid $(\mathrm{q}=4.074, \mathrm{p}<0.05)$. We found a significant difference between groups for carbonyls $(\mathrm{F}(3,16)=10.63, \mathrm{p}<0.001)$ (Figure 6.3 B). Tukey's post-hoc comparison showed a significant difference between control and blast $(\mathrm{q}=6.429, \mathrm{p}<0.01)$, lipoic acid and blast $(\mathrm{q}=4.226, \mathrm{p}<0.05)$, and blast and blast + lipoic acid $(\mathrm{q}=7.302, \mathrm{p}<0.001)$. Lipoic acid significantly reduced oxidative stress byproducts.

\section{A decrease in anti-apoptotic markers is seen 24 hours post-blast but lipoic acid restores}

\section{towards baseline}

Western blot investigation showed significant changes to anti-apoptotic makers in the left prefrontal cortex following a single blast exposure. Bcl-2 is an important antiapoptotic oncogene that provides neuroprotection when increased (Indharty 2013). A significant difference was seen between groups 24 hours post-blast $(F(3,12)=4.637$, $\mathrm{p}<0.05)$. Post-hoc comparison revealed that single blast exposure significantly decreased Bcl-2 compared to lipoic acid only $(q=3.079, \mathrm{p}<0.05)$ (Figure 6.4 A). The multiple comparisons between other groups showed no significant difference. Heme oxygenase 1 (HO-1) is an inducible enzyme that degrades toxic heme following oxidative stress. Pharmacologic upregulation of HO-1 following TBI leads to improved neuronal survival by preventing apoptosis (Ding et al 2014). A significant difference was seen between groups for HO-1 24 hours post-blast $(F(3,12)=11.33, \mathrm{p}<0.001)$. Post-hoc comparison revealed that lipoic acid only increased the level of HO-1 expression when compared to control $(\mathrm{q}=4.826, \mathrm{p}<0.05)$ (Figure 6.4 B). Post-hoc comparison also showed that the 
expression of HO-1 decreased significantly between the lipoic acid only and single blast $(\mathrm{q}=8.169, \mathrm{p}<0.001)$ animals. The level of expression also increased significantly between single blast and single blast treated with lipoic acid groups $(\mathrm{q}=4.417, \mathrm{p}<0.05)$ back towards control levels. The overall increase in anti-apoptotic markers following lipoic acid treatment suggests a protective mechanism limiting Nox4-induced oxidative stress damage.

\section{Mild changes in pro-apoptotic markers were seen 24 hours post-blast}

Fluorescent immunohistochemistry of the left prefrontal cortex showed mild changes of pro-apoptotic markers 24 hours following single blast exposure. Bax is the pro-apoptotic regulator of Bcl-2 that is often increased following TBI (Ghadiri et al 2014). Not surprisingly, a significant difference between groups was seen for Bax $(F(2,27)=3.604, p<0.05)$. Post-hoc comparison revealed a significant difference between animals exposed to a single blast compared to control $(\mathrm{q}=3.724, \mathrm{p}<0.05)$ (Figure $6.5 \mathrm{~A})$. No other significant expression changes were seen between the other experimental groups. Caspase 12 is a pro-apoptotic marker that can lead to the activation of caspase 3. A significant difference was seen between groups $(F(2,27)=4.325, p<0.05)$. Post-hoc comparison revealed a decrease in the expression of caspase 12 in animals exposed to a single blast with alpha lipoic acid treatment compared to control $(q=4.056, p<0.05)$ (Figure 6.5 B). No other changes in expression were seen between any other groups. Caspase 3 is an important down stream regulator of apoptosis. If caspase 3 is active, apoptosis will occur. No significant change in caspase 3 expression was seen between experimental groups $(\mathrm{F}(2,33)=1.985, \mathrm{p}=0.1535$ (Figure 6.5 C). 
No appreciable difference in anti-apoptotic markers were observed 2 weeks post repeat

$\underline{\text { blast }}$

Repeat injury is a growing concern in the field of neurotrauma. Repetitive concussions have been linked to CTE and other tauopathies (Lucke-Wold et al 2014c). An area in need of further investigation is the development of treatment options to prevent chronic neurodegeneration following acute TBI. We sought to investigate the beneficial effects of lipoic acid administration given 5 minutes after each blast ( 6 blasts total every other day for 2 weeks). Western blot analysis of the left prefrontal cortex was performed to determine expression changes in anti-apoptotic markers two weeks after repetitive blast exposure. No significant difference between groups was observed for Bcl$2(\mathrm{~F}(3,12)=2.538, \mathrm{p}=0.1057)($ Figure 6.6 A). Likewise, no significant difference was observed between groups for HO-1 $(\mathrm{F}(3,12)=0.2129, \mathrm{p}=0.8855)($ Figure 6.6 B). Expression of all markers was shown to remain consistent across all treatment groups. The data support that no changes in anti-apoptotic markers were seen sub-acutely postrepeat injury.

\section{Lipoic acid reduced pro-apoptotic markers 2 weeks post-repetitive blast}

Apoptosis is an important indicator of neurodegeneration (Tajes et al 2014). We examined three pro-apoptotic markers in the left prefrontal cortex two weeks following repetitive blast. A significant difference was seen in the expression of Bax between groups $(\mathrm{F}(2,27)=7.93, \mathrm{p}<0.01)$. Post hoc comparison showed an increase in Bax with repetitive blast animals compared to control $(\mathrm{q}=5.194, \mathrm{p}<0.01)$, but the increase was ameliorated when lipoic acid was given after each blast $(\mathrm{q}=4.483, \mathrm{p}<0.05)$ (Figure 6.7 A). No significant difference existed between the control and repetitive blast treated with 
alpha lipoic acid groups. A significant difference between groups was noted for caspase $12(\mathrm{~F}(2,27)=11.96, \mathrm{p}<0.001)$. Post-hoc comparison revealed a significant increase in expression between repetitive blast animals compared to control $(\mathrm{q}=5.809, \mathrm{p}<0.001)$, but lipoic acid administration after each blast negated this response $(\mathrm{q}=6.157, \mathrm{p}<0.001)$ (Figure 6.7 B). No significant difference existed between the control and repetitive blast treated with alpha lipoic acid groups. A significant difference in caspase 3 expression was noted between groups $(\mathrm{F}(2,27)=12.6, \mathrm{p}<0.001)$. Post-hoc comparison revealed a significant increase in caspase 3 for animals exposed to repetitive blast compared to control animals $(q=6.989, p<0.001)$, but repetitive blast animals treated with alpha lipoic acid had a reduced response $(\mathrm{q}=4.573, \mathrm{p}<0.01)$ (Figure $6.7 \mathrm{C})$. Lipoic acid effectively decreases expression of pro-apoptotic markers at subacute time points ( 2 weeks) following repetitive blast.

\section{NADPH oxidase markers and pro-apoptotic markers are increased in human CTE} samples

We examined the NADPH markers, Nox4 and p22phox, in human CTE samples obtained from a professional football player and professional wrestler that were then compared to an age and sex matched control. A visible increase in Nox4 was observed in the football player (32/100) and wrestler (27/100) samples compared to control (6/100) with $\chi^{2}=26.527, \mathrm{p}<0.0001$ (Figure $6.8 \mathrm{~A}$ ). Likewise, a visible increase in p22phox was observed for the football player (17/100) and wrestler (24/100) samples compared to control (3/100) with $\chi^{2}=34.585, \mathrm{p}<0.0001$ (Figure $6.8 \mathrm{~B}$ ). The visual evidence suggests that concussive injuries lead to an increase in the expression of NADPH markers at extended time points. This increase is likely associated with the production of free 
radicals that can lead to permanent tissue damage. The response to tissue damage in a diseased brain is often impaired. A significant difference between groups was observed for the anti-apoptotic marker Bcl-2 $(\mathrm{F}(2,27)=23.8, \mathrm{p}<0.001)$. Post hoc comparison was significant between football player and control $(q=7.302, p<0.001)$, and between wrestler and control $(q=9.255, \mathrm{p}<0.001)$ (Figure 6.9 A). Neurofibrillary tangles have been proposed to spread between intact cells. These cells must be able to avoid apoptosis when injured. A significant difference was also seen between groups for the proapoptotic marker $\operatorname{Bax}(F(2,27)=23.38, \mathrm{p}<0.001)$. Post hoc comparison was significant between football player and control $(q=5.424, p<0.01)$, between wrestler and control ( $q$ $=4.221, \mathrm{p}<0.05)$, and between football player and wrestler $(\mathrm{q}=9.645, \mathrm{p}<0.001)$ (Figure 6.9 B). The football player case was a less severe form of CTE indicating that apoptosis may play a role in the early disease stages. The wrestler case had severe CTE pathology and showed that apoptosis maybe less likely to play a role in late disease stages. Tau pathology increased following repetitive blast in rodents and in human CTE specimens

Following injury, tau becomes hyperphosphorylated and can undergo conformational changes leading to neurofibrillary tangles. $\mathrm{CP}-13$ is a marker of pretangle tau whereas MCI is a conformational configuration of tau seen primarily in neurodegenerative disease. Paired helical filament is a dimerized form of tau that precedes the formation of oligomers. A significant difference between groups was observed for CP-13 in blast exposed animals $(F(2,27)=8.15, \mathrm{p}<0.01)$. Post hoc comparison showed a significant increase in $\mathrm{CP}-13$ for the repeat blast group compared to control $(\mathrm{q}=5.54, \mathrm{p}<0.01)$, but lipoic acid ameliorated this change when administered 
post blast $(\mathrm{q}=3.967, \mathrm{p}<0.05)$ (Figure 6.10 A). A significant difference was not observed between groups for $\mathrm{MCI}$ in blast exposed animals $(\mathrm{F}(2,27)=2.492, \mathrm{p}=0.1016)$ (Figure 6.10 B). A significant difference between groups was observed for PHF in blast exposed animals $(F(2,27)=18.01, p<0.001)$. Post hoc comparison showed a significant increase in PHF for the repeat blast group compared to control $(q=7.865, p<0.001)$, but lipoic acid ameliorated this change when administered post blast $(\mathrm{q}=6.703, \mathrm{p}<0.001)$ (Figure 6.10 C). A significant difference was observed for $\mathrm{CP}-13$ in human specimens $(\mathrm{F}(2,27)=$ 20.31, $\mathrm{p}<0.001)$. Post hoc comparison revealed a significant difference between the football player and control $(\mathrm{q}=6.406, \mathrm{p}<0.001)$, and between the wrestler and control (q $=8.695, \mathrm{p}<0.001)($ Figure $6.11 \mathrm{~A})$. A significant difference was observed for MCI in human specimens $(F(2,27)=24.41, p<0.001)$. Post hoc comparison revealed a significant difference between the football player and control $(\mathrm{q}=9.88, \mathrm{p}<0.001)$, and between the wrestler and control $(\mathrm{q}=4.838, \mathrm{p}<0.01)$ (Figure $6.11 \mathrm{~B})$. A significant difference was observed for PHF in human specimens $(\mathrm{F}(2,27)=51.21, \mathrm{p}<0.001)$. Post hoc comparison revealed a significant difference between the football player and control $(\mathrm{q}=10.58$, $\mathrm{p}<0.001)$, and between the wrestler and control $(\mathrm{q}=13.64, \mathrm{p}<0.001)$ (Figure 6.11 C).

\section{Blast injury results in an increase of impulsivity-like behavior}

Elevated plus maze has been used as a test for impulsivity (Kim et al 2012b). Animals spending more time in the open arm exhibit decreased risk aversion. The closed arms represent a darkened environment, which is a preference for nocturnal rodents. A significant difference between groups was noted for time spent in the open arm at 7 days post repetitive blast $(\mathrm{F}(2,21)=4.327, \mathrm{p}<0.05)$. Post-hoc comparison revealed that rats exposed to repetitive blast exhibited an increase in impulsive-like behavior spending 
more time in the open arms compared to the control group $(\mathrm{q}=3.632, \mathrm{p}<0.05)$, and repetitive blast + lipoic acid treatment group brought the exploratory behavior back towards control levels $(\mathrm{q}=3.573, \mathrm{p}<0.05)($ Figure $6.12 \mathrm{~A})$. We wanted to test if animals receiving repetitive blast were overly active by measuring total distance traveled. Notably, no difference was observed between groups for overall distance traveled during the 5-minute interval within the apparatus $(\mathrm{F}(2,21)=0.3174, \mathrm{p}=0.7315)($ Figure $6.12 \mathrm{~B})$.

\section{DISCUSSION}

We show for the first time that alpha-lipoic acid reduced Nox4 and p22phox following blast TBI. Alpha-lipoic acid acutely increased anti-apoptotic markers 24 hours after injury. In addition, alpha-lipoic acid decreased pro-apoptotic markers both acutely and sub-acutely following blast exposure as well as decreasing tau hyperphosphorylation. Animals exposed to repeat blast exposure had increased impulsive-like behavior on elevated plus maze (EPM) that was decreased when alpha-lipoic acid was administered following blast. Alpha lipoic acid, a naturally available supplement, has been used in previous neural injury studies for its role as an anti-oxidant and for decreasing neuroinflammation (Rocamonde et al 2013, Rocamonde et al 2012, Toklu et al 2009). It has recently been suggested that lipoic acid would be a promising therapeutic for TBI treatment, but experimental preclinical studies are limited (Hall et al 2010). We investigated for the first time the beneficial effects of alpha lipoic acid on NADPH oxidative stress reduction following blast TBI. We hypothesize that alpha lipoid acid reduces Nox4 complex formation with p22phox. Because lipoic acid does not cross the mitochondrial membrane, we propose it acts peripherally at the neuronal membrane to decrease superoxide formation, carbonyls, and 8-hydroxy-2-deoxyguanosine. The free 
radical damage is likely due to surrounding microglia activation and peripheral immune infiltration.

The blast exposure set up used for this study has previously been shown to produce substantial neural injury to the left cortex due to acceleration/deceleration dynamics with little damage to the right cortex (Turner et al 2013). We provide reasonable evidence that alpha lipoic acid reduces the NADPH oxidase Nox4 in the contralateral left prefrontal cortex, and subsequently inhibits the Nox4 complex formation with p22phox at 24 hours post-blast. Nox 4 has been shown to peak near contusion sites in humans $24-48$ hours following TBI ( $\mathrm{Li}$ et al 2014b). Nox4 is predominately located in neurons and is mediated by specific inducible mRNA (Peng et al 2013). The Nox4 pathway can produce toxic free radicals that may lead to cellular apoptosis ( $\mathrm{Li}$ et al 2014a). We observed a significant reduction in superoxide, carbonyls, and 8-hydroxy-2-deoxyguanosine but not total ROS. Lipoic acid passes through the neuronal membrane but not the mitochondrial membrane (Wada et al 1997). ROS generated by mitochondria are not the primary target of lipoic acid. Preventing Nox4 activation may therefore have profound effects on reducing neural injury expansion and oxidative stress byproducts over time at the plasma membrane. Inhibiting another NADPH oxidase, Nox2, following TBI has already proved promising in previous studies with apocynin (Choi et al 2012, Ferreira et al 2013). Continued investigations into the Nox4 and Nox2 pathways and their relation to TBI are warranted.

Not surprisingly, lipoic acid treatment increased expression of the anti-apoptotic markers, Bcl-2 and HO-1, 24 hours following single blast injury. Enhancing Bcl-2 activity following TBI has been shown to decrease apoptosis and improve cognitive 
performance (Leeds et al 2014). Likewise, increasing HO-1 activity facilitates the degradation of toxic heme and prevents plasma membrane damage and the induction of inflammatory cascades (Okubo et al 2013). HO-1 also has been shown to reduce toxic iron mediated damage likely accounting for the decrease in free iron observed in the lipoic acid treated group (Wagener et al 2013). HO-1 converts toxic iron protoporphyrin into stable $\mathrm{Fe}(2+)$ that can be readily cleared from the brain (Gozzelino et al 2010). At two weeks following repetitive blast (6 blasts over the time course of 2 weeks), lipoic acid treatment decreased pro-apoptotic markers Bax, caspase 12, and caspase 3. Bax and caspase 3 trigger rapid and sustained apoptosis following TBI (Ghadiri et al 2014). Bax deactivates Bcl-2 while caspase 3 triggers the final common pathway of apoptosis. Caspase 12 is readily increased when toxic free radicals damage the endoplasmic reticulum (Liu et al 2014b). Caspase 12 can interact with pro-apoptotic factors released from damaged mitochondria to facilitate activation of caspase 3 (Chen et al 2013). Reducing pro-apoptotic markers following TBI can decrease neurodegeneration (Supplementary Figure 6.1). We showed that lipoic acid reduced pathologic conformational changes of tau following repetitive blast.

The link between apoptosis and subsequent tauopathy is likely due to chronic neuroinflammation. A recent study suggests that peripheral immune cells enter the brain via a caveolin dependent mechanism (Badaut et al 2015). Caveolin increases ICAM-1 binding affinity for leukocytes post-injury causing increased diapedesis across damaged endothelial cells (Xu et al 2013). The peripheral immune cells that enter the brain release cytokines that trigger a robust gliosis response (Freeman \& Ting 2015). The inflammatory storm can cause activation of NADPH oxidases, which further damage 
neurons and contribute to apoptosis (Ho et al 2015). Overtime nuclear factor kappa B is translocated to the nucleus triggering a chronic state of inflammation (Ahmad et al 2013). This chronic inflammation can contribute to the formation of tauopathy (Breunig et al 2013). By targeting NADPH oxidase activity, lipoic acid can be used to disrupt this cycle. Further studies are warranted to investigate the long-term effects of targeting NADPH activity on chronic neuroinflammation. We provide evidence that lipoic acid improves behavior following multiple injuries.

A behavioral alteration often seen in soldiers exposed to blast injury is impulsivity (James et al 2014). Impulsivity has been linked to brain degeneration and tauopathy in human CTE (Stein et al 2014). Impulsivity and aggression can lead to personal relationship and work problems (Booth-Kewley et al 2010). In severe cases, impulsivity can lead to death due to unnecessary risk-taking behavior (Lopez-Castroman et al 2014). A behavioral measure of impulsive-like behavior in rodents is the elevated plus maze (Steimer \& Driscoll 2003). Rats with impulsive tendencies spend increased time in the open arms of the maze. We observed a significant increase in impulsive-like behavior that was ameliorated by lipoic acid administration 7 days following repetitive blast injury. Repetitive blast injury did not however increase overall mobility. The mechanism behind lipoic acid's beneficial effects is likely due to its anti-oxidant and anti-apoptotic properties. Future research will examine how lipoic acid affects cognitive function and apoptosis within the hippocampus. The current findings correlate well with the tauopathy changes we report. By decreasing apoptosis, lipoic acid can potentially mitigate the effects of tauopathy and prevent the chronic sequelae of associated neurodegenerative symptoms. 
CTE is an important neurodegenerative disease of growing prevalence and importance, yet the pathophysiology is still poorly understood. A lack of prospective studies and post-mortem diagnosis limits investigation into disease progression and treatment targets (Baugh et al 2014). The use of new PET scans with a radioligand, which labels both amyloid and tau, is improving diagnostic capabilities for living individuals but further work needs to be done (Small et al 2013). We present for the first time that the Nox4/p22phox pathway may play an important role in CTE pathology. Human samples from a professional football player and wrestler had increased levels of Nox4 and p22phox in comparison to age and gender-matched controls. Additionally, the CTE samples showed disrupted apoptotic signaling mechanisms and abundant tauopathy. Bcl2 was significantly increased in both the football player and wrestler samples. Pure tauopathies, unlike amyloid $\beta$ mediated Alzheimer's disease, have decreased apoptosis in late stages of the disease. Hyperphosphorylated tau increases Bcl-2 allowing for effective transfer of neurofibrillary tangles between living cells (Wang et al 2010). Tauopathies also often have reduced pro-apoptotic markers. We observed that while Bax was increased in the football player brain, it was reduced in the more severely affected wrestler brain. The wrestler brain had neurofibrillary tangles and neuropil threads throughout the cortex, deep nuclei, and brain stem indicating the most advanced stage of CTE (Omalu et al 2010b). As long as tau phosphatases are still active, tau dephosphorylation can cause an increase in Bax and trigger apoptosis in injured cells (Liu et al 2010). At some point during the progression of CTE, tau phosphatases may decrease substantially and the levels of Bax are markedly reduced. Nox4 fits into the picture by producing free radicals that can derange signaling pathways. These deranged pathways 
typically trigger apoptosis, but in neurodegenerative disease they lead to hyperphosphorylation of tau and conformational changes (Giraldo et al 2014). Hyperphosphorylated tau acts in a positive feedback loop ramping up oxidative stress (Borza 2014).

Lipoic acid may therefore have multiple beneficial effects when used as a treatment for neurotrauma. Acutely, lipoic acid could decrease apoptosis by limiting oxidative stress. Chronically after CTE has developed, lipoic acid could target the positive tau feedback loop limiting excess tau hyperphosphorylation and conformational change thereby slowing the progression of CTE. Many studies remain to be done in order to investigate the beneficial properties of lipoic acid at chronic time points. Continued supplementation following injury will probably have the most successful results. In order to advance towards clinical trial, it will be necessary to utilize models that are more representative of human disease pathology. Approaches such as a transgenic animals or the use of a species closely related to humans on the evolutionary tree will likely be necessary. Additionally, scaling blast injury for these new species must be considered in order to accurately represent human injury exposure. Clinical safety studies are advancing for lipoic acid's use in the treatment of other disorders (Kates et al 2014). Considerations regarding lipoic's acid use for neurotrauma must be focused upon blood brain barrier transport, effective dosing, and appropriate timing of administration.

\section{CONCLUSIONS}

In summary, lipoic acid provided acute neuroprotection in our clinically relevant and validated blast TBI model. It decreased Nox4 and p22phox while increasing antiapoptotic markers acutely. Subacutely it decreased pro-apoptotic markers, decreased tau 
conformational changes, and improved impulsive behavior. CTE samples from a football player and wrestler also showed signs of increased Nox 4 activity. The Nox4 pathway produces hydrogen peroxide and superoxide radicals that damage mitochondria, cellular DNA, proteins, and lipid membranes (Lobo et al 2010). Long-term consequences include neurodegenerative disease, tauopathy, and neuropsychiatric changes. Considering the limitations to diagnosis and the incomplete understanding of CTE pathophysiology, Nox4 mediated oxidative stress should be more closely investigated. Oxidative stress contributes to an important interplay with tau hyperphosphorylation. Ongoing projects conducted by our laboratory will investigate the Nox4 pathway as well as lipoic acid modulation at extended time points. Lipoic acid is a widely used supplement that has already begun to be evaluated in clinical trials for the treatment of other chronic disorders. Lipoic acid might be a worthwhile treatment option for TBI as well and potentially offers a break through for an injury paradigm that has limited treatment options. The mechanism of lipoic acid neuroprotection are not fully known and must be further investigated going forward.

\section{ACKNOWLEDGMENTS}

All authors have read the journal's authorship agreement and the manuscript has been reviewed and approved by all named authors. The authors have no conflicts of interest to declare, and have read the journal's policy on potential conflicts of interest. A West Virginia University Research and Development Grant funded the current project. An American Medical Association Foundation Seed Grant, an American Foundation of Pharmaceutical Education Pre-doctoral Fellowship, and a Neurosurgery Research and Education Foundation Medical Student Summer Research Fellowship were awarded to 
support Brandon Lucke-Wold. Aric Logsdon also received an American Foundation of Pharmaceutical Education Pre-doctoral Fellowship. Editorial Support was not received for the preparation of the manuscript. 


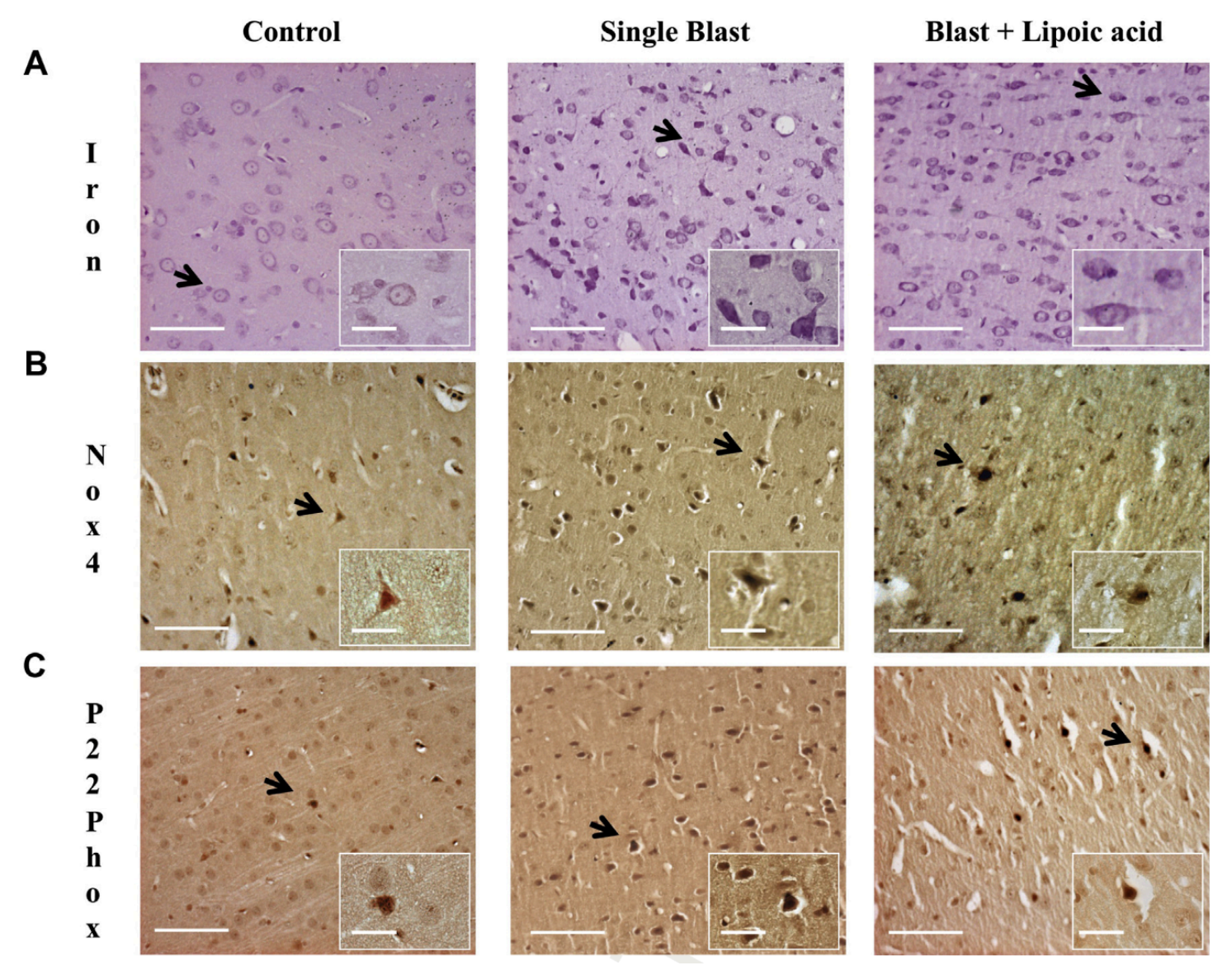

Figure 6.1: Increased intracellular iron and NADPH oxidase in the left prefrontal cortex 24 hours post-blast. Scale bar for whole images $=50 \mu \mathrm{m}$. Scale bar for inlay images $=$ $20 \mu \mathrm{m}$. (A) The amount of intracellular iron accumulation increased 24 hours following single blast (19/100) compared to control (2/100). When alpha lipoic acid was given 5 minutes post-blast the amount of intracellular iron decreased (11/100) compared to single blast indicating preserved plasma membrane integrity. (B) DAB staining for Nox4 shows an increase in expression in the left prefrontal cortex 24 hours following single blast $(29 / 100)$ compared to control (8/100). The increased level of Nox4 suggests an elevated production of reactive oxygen species. Alpha lipoic acid treatment decreased Nox4 back towards control levels (12/100). (C) DAB staining of p22phox, which forms an activation 
complex with Nox4, showed increased expression in single blast (23/100) compared to control (6/100). Alpha lipoic acid treatment 5 minutes post-blast decreased p22phox (13/100) back towards control levels. 
A

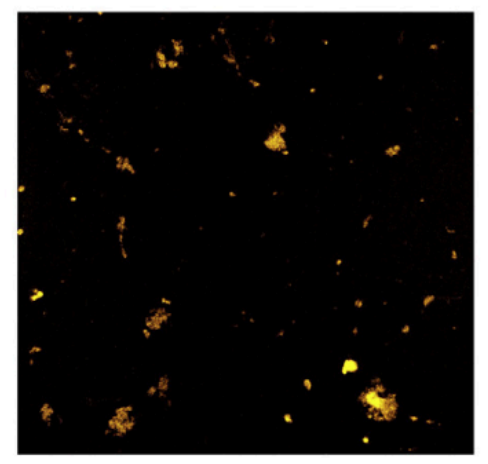

B
Superoxide

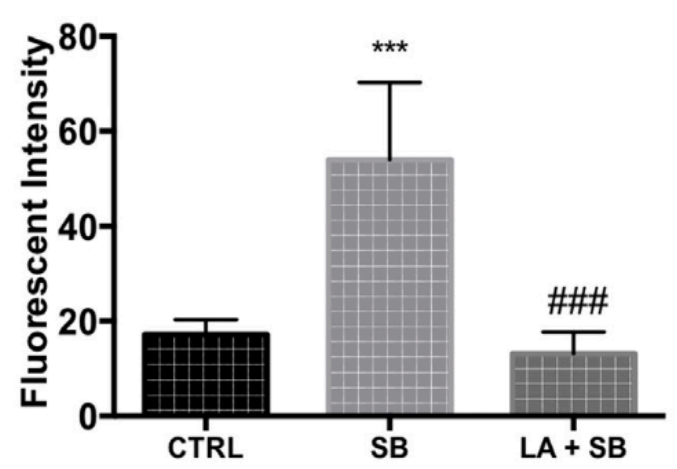

SB

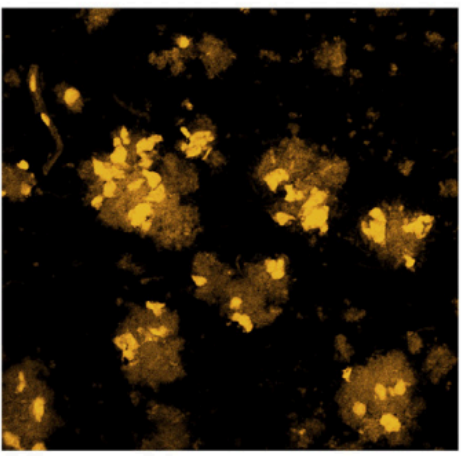

C

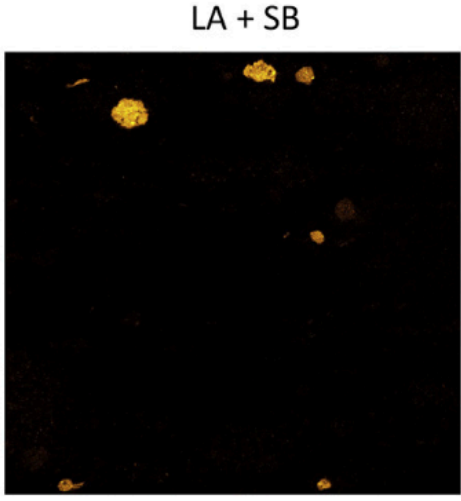

Total ROS

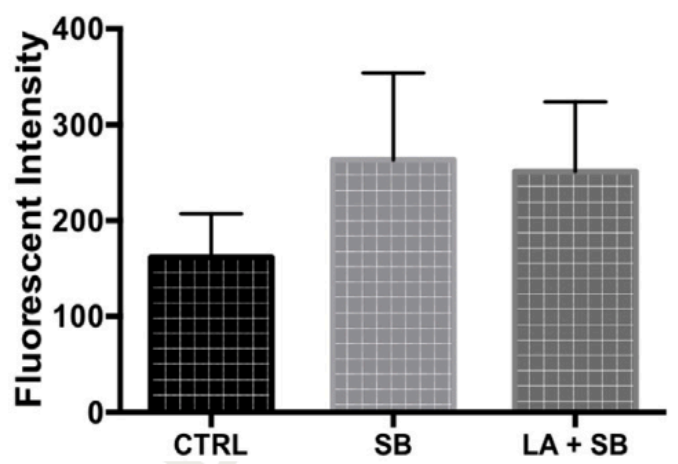

Figure 6.2: Lipoic acid reduces the generation of superoxide in the left prefrontal cortex post-blast. The Nox4 pathway can lead to the generation of free radicals at the plasma membrane. In particular, NADPH leads to generation of superoxide. This radical can damage intracellular organelles, which contributes to apoptosis. (A) A visual representation of superoxide staining shows an increase following single blast that is reduced by lipoic acid administration post blast. (B) A significant increase in superoxide between single blast and control groups was detected with fluorescent scanning at 24 hours post-blast $(\mathrm{q}=9.01, \mathrm{p}<0.001)$ but was ameliorated by lipoic acid administration ( $\mathrm{q}$ $=10.09, \mathrm{p}<0.001) . * * *=p<0.001 \mathrm{SB}$ vs. CTRL. \#\#\#=p<0.001 SB vs. LA + SB. No significant changes were reported for total reactive oxygen species. 
A
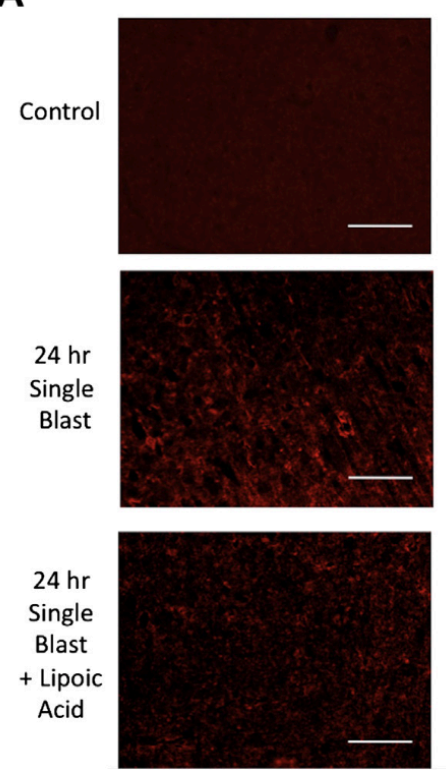
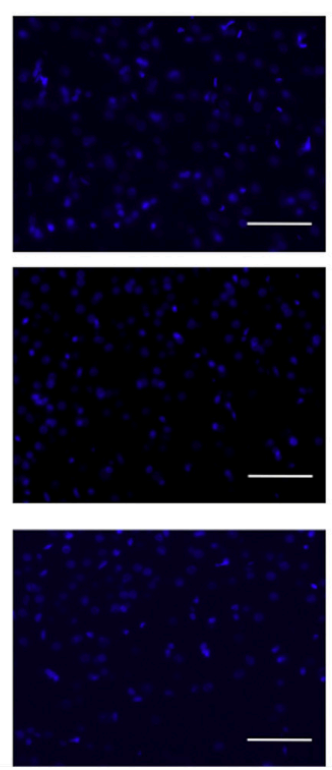

B
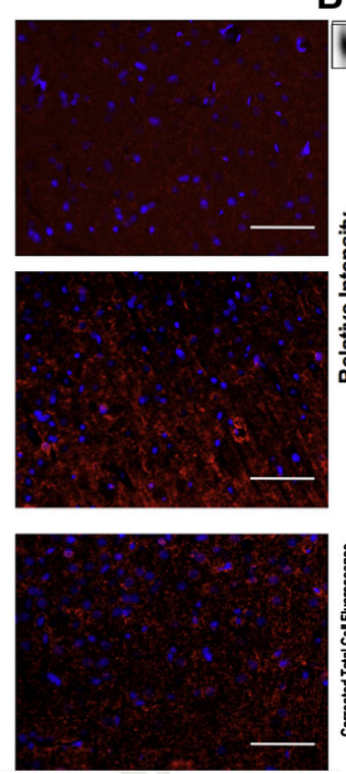

Std C LA SB SB

Carbonyls $+\mathrm{LA}$
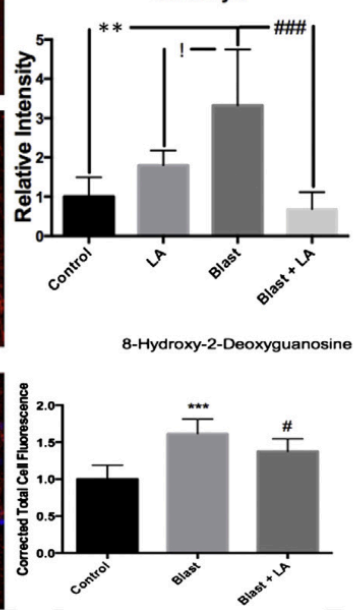

Figure 6.3: Lipoic acid significantly reduces oxidative stress byproducts. 8-hydroxy-2deoxyguanosine and carbonyls have been showng to be increased following TBI. (A) a significant increase in 8-hydroxy-2-deoxyguanosine was seen 24 hours post blast compared to control $(\mathrm{q}=10.53, \mathrm{p}<0.001)$. Lipoic acid administration prevented this increase $(q=4.074, p<0.05)$. (B) a significant increase in carbonyls was seen between control and blast $(\mathrm{q}=6.429, \mathrm{p}<0.01)$. Other significant comparisons were seen between the lipoic acid and blast groups $(\mathrm{q}=4.226, \mathrm{p}<0.05)$ and the blast group and blast + lipoic acid group $(\mathrm{q}=7.302, \mathrm{p}<0.001) . *$ =significance between control and blast, !=significance between lipoic acid and blast, and \#=significance between blast and blast + lipoic acid. 
A

\section{Bcl-2}
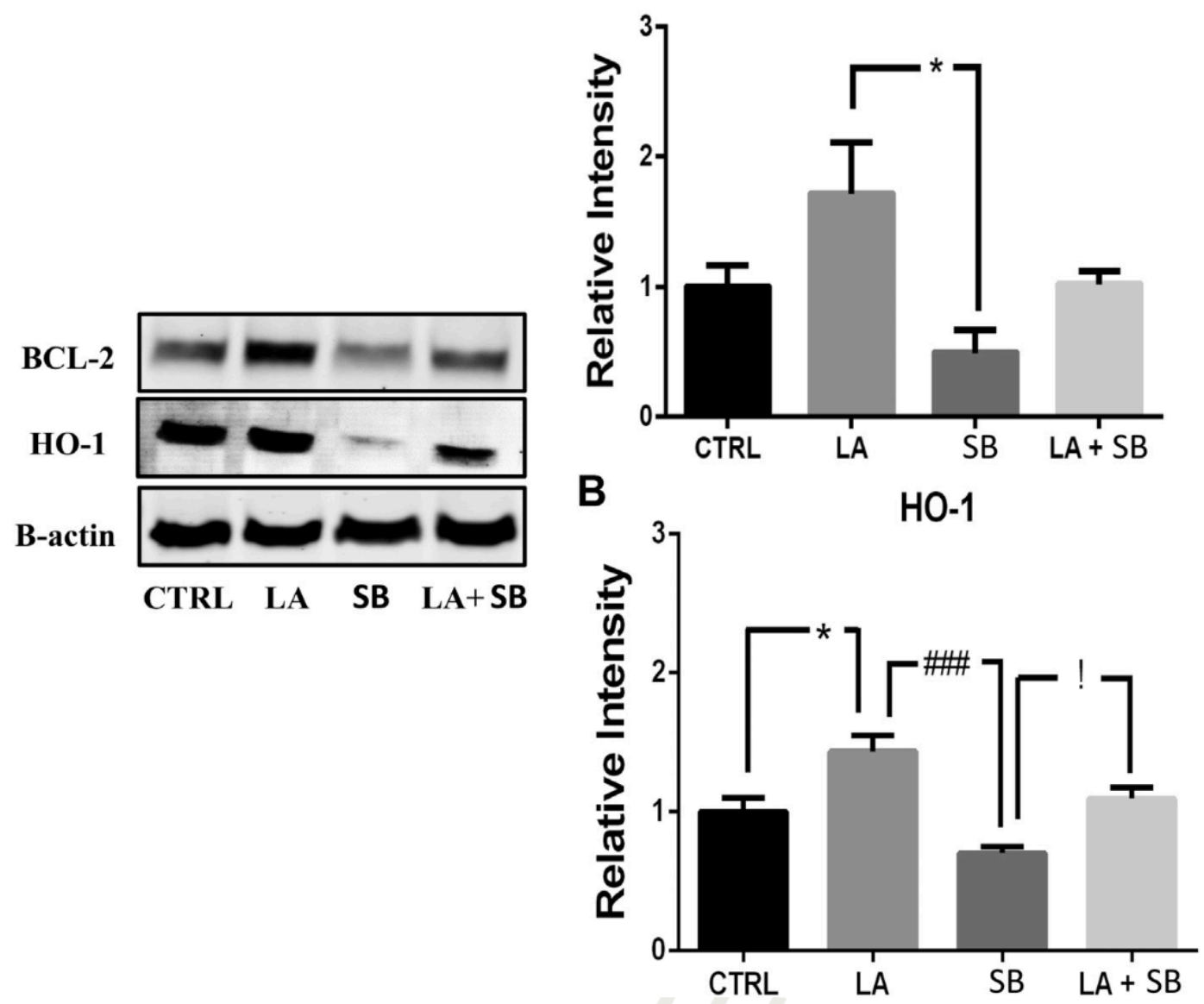

Figure 6.4: Anti-apoptotic markers from left prefrontal cortex 24 hours post-injury. (A) A decrease in Bcl-2 expression was seen in single blast compared to lipoic acid only $(\mathrm{q}=$ $3.079, \mathrm{p}<0.05)$. No significant difference is seen when comparing other experimental groups. ${ }^{*} \mathrm{p}<0.05$ LA vs. SB. (B) Significant differences were seen between groups when probing for Heme Oxygenase 1 (HO-1). An increase in $\mathrm{HO}-1$ was seen between control compared to lipoic acid only $(q=4.826, p<0.05)$. A decrease was seen between lipoic acid only and single blast $(\mathrm{q}=8.169, \mathrm{p}<0.001)$, and an increase was seen between single blast compared to single blast treated with lipoic acid $(\mathrm{q}=4.417, \mathrm{p}<0.05)$. No significant difference was seen when comparing the other experimental groups. ${ }^{*} p<0.05$ CTRL vs. 
LA. \#\#\# p $<0.001$ LA vs. SB. ! p $<0.05$ SB vs. LA + SB. CTRL $=$ control, LA = lipoic acid, SB = single blast. 
A

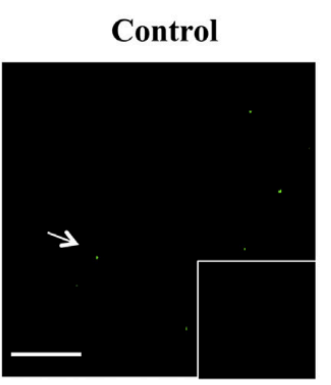

B

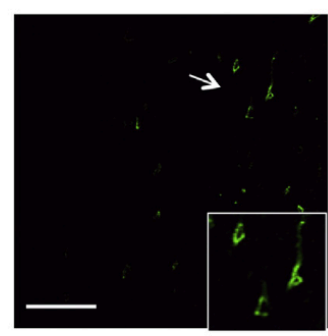

C

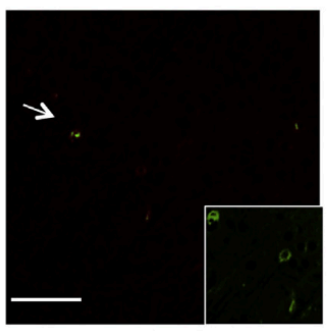

Single Blast
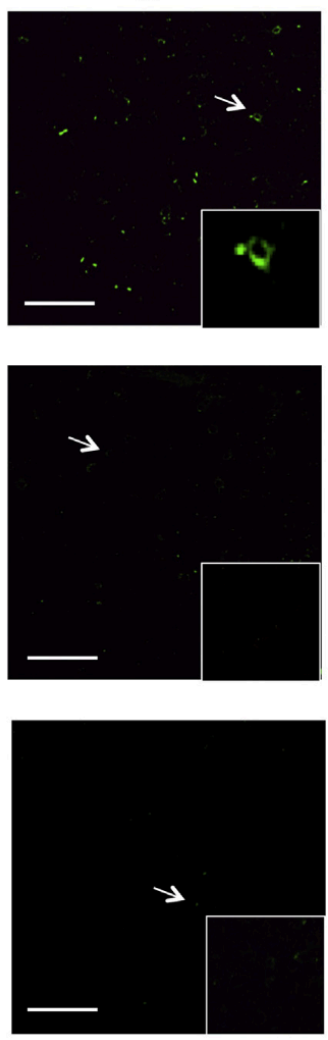

Blast + Lipoic acid
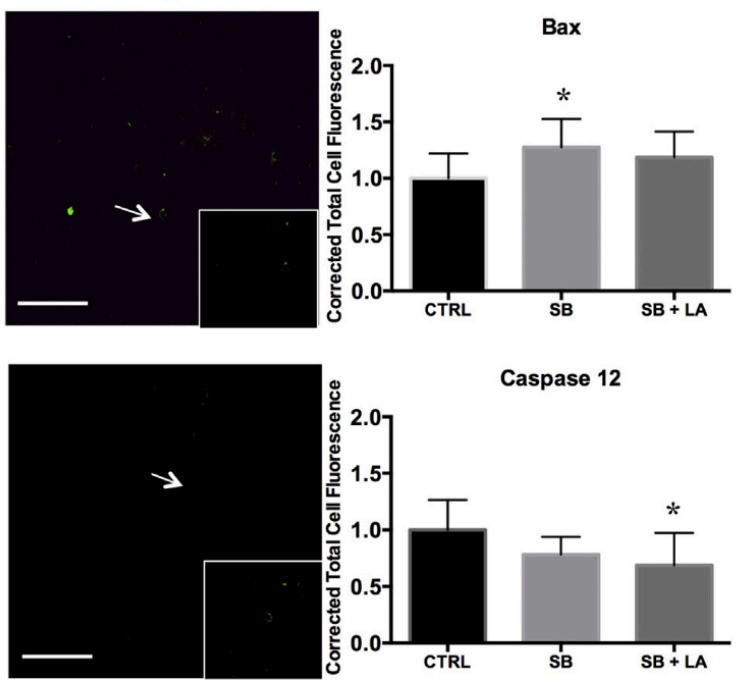

Caspase 3
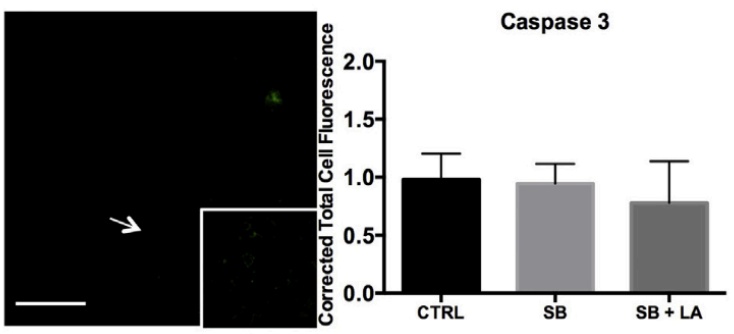

Figure 6.5: Immunohistochemistry of pro-apoptotic markers in the left prefrontal cortex at 24 hours post-blast. Scale bar $=100 \mu \mathrm{m}$. (A) Bax expression is increased in animals exposed to single blast compared to control $(\mathrm{t}=3.724, \mathrm{p}<0.05)$. No change is seen between the other groups. ${ }^{*} \mathrm{p}<0.05$ CTRL vs. SB. (B) A decrease is seen in the expression of caspase 12 in animals treated with alpha lipoic acid following blast compared to control $(\mathrm{t}=4.056, \mathrm{p}<0.05)$. No change is seen between other experimental groups. ${ }^{*} \mathrm{p}<0.05$ CTRL vs. SB + LA. (C) Caspase 3 expression shows no change between experimental groups. $\mathrm{CTRL}=$ control, $\mathrm{SB}=$ single blast, $\mathrm{LA}=$ lipoic acid. 


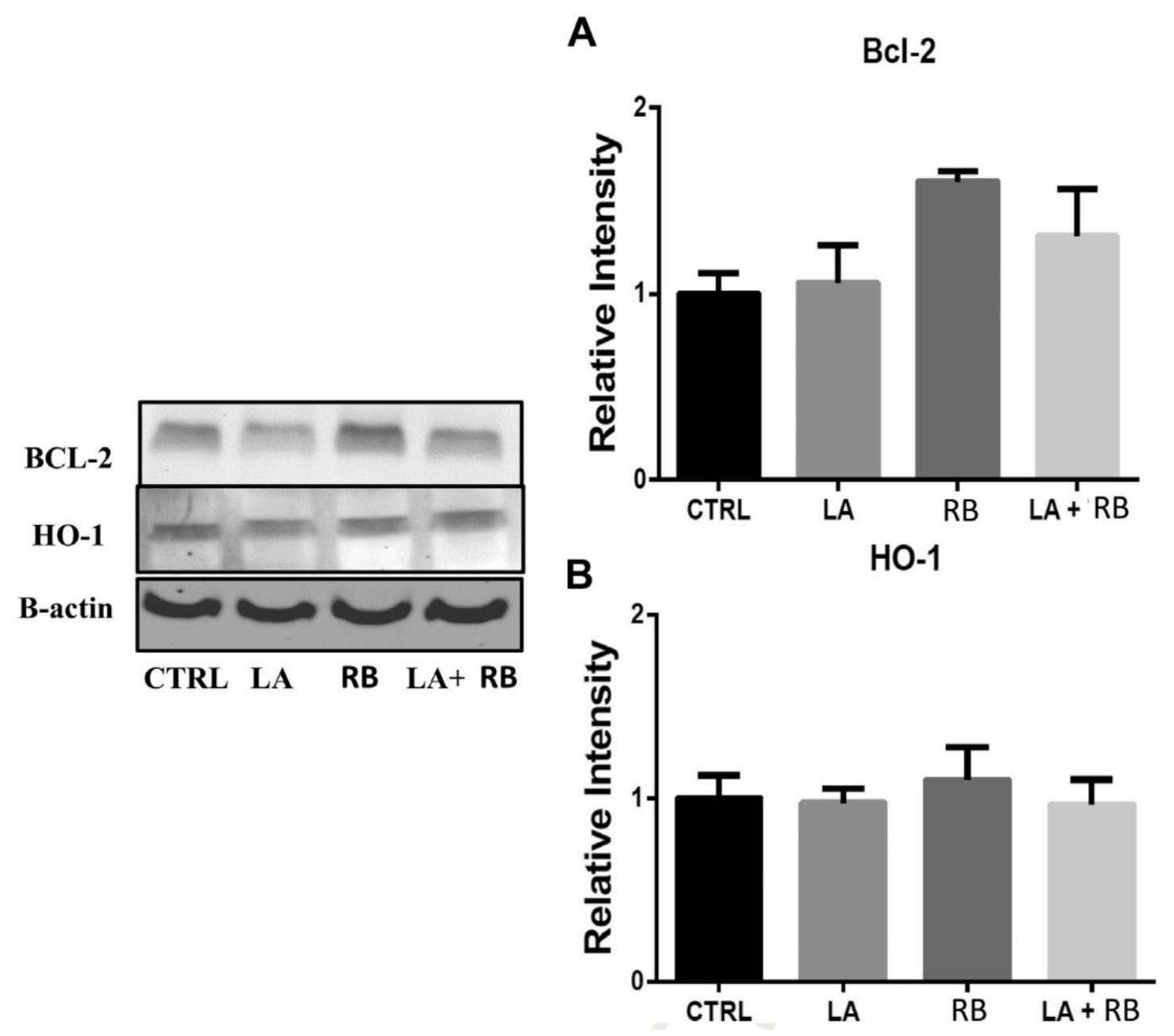

Figure 6.6: Immunoblot of anti-apoptotic markers in the left prefrontal cortex 2 weeks post repetitive blast exposure. Animals were exposed to 6 blasts total over the time course of two weeks. No significant differences between groups were observed for (A) Bcl-2 or $(\mathbf{B})$ HO-1. CTRL $=$ control, $\mathrm{LA}=$ lipoic acid, $\mathrm{RB}=$ repeat blast, $\mathrm{LA}+\mathrm{RB}=$ lipoic acid treatment 5 minutes post each blast, Bcl-2 = B-cell lymphoma 2, and HO-1 = heme oxygenase 1 . 
A

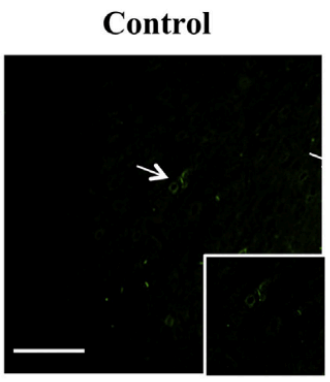

B

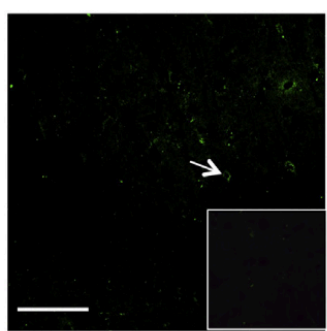

C

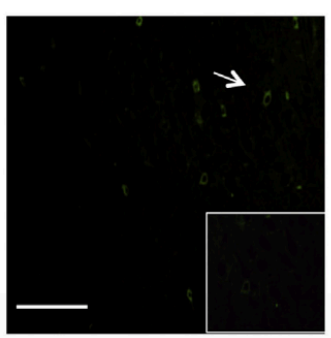

Repeat Blast
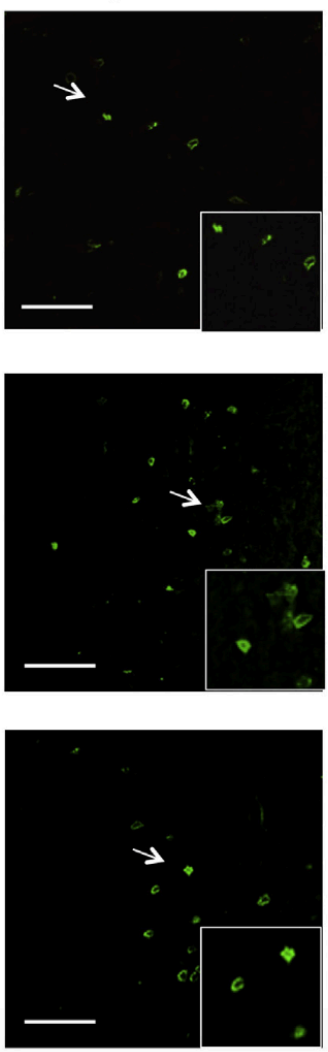

Blast + Lipoic acid
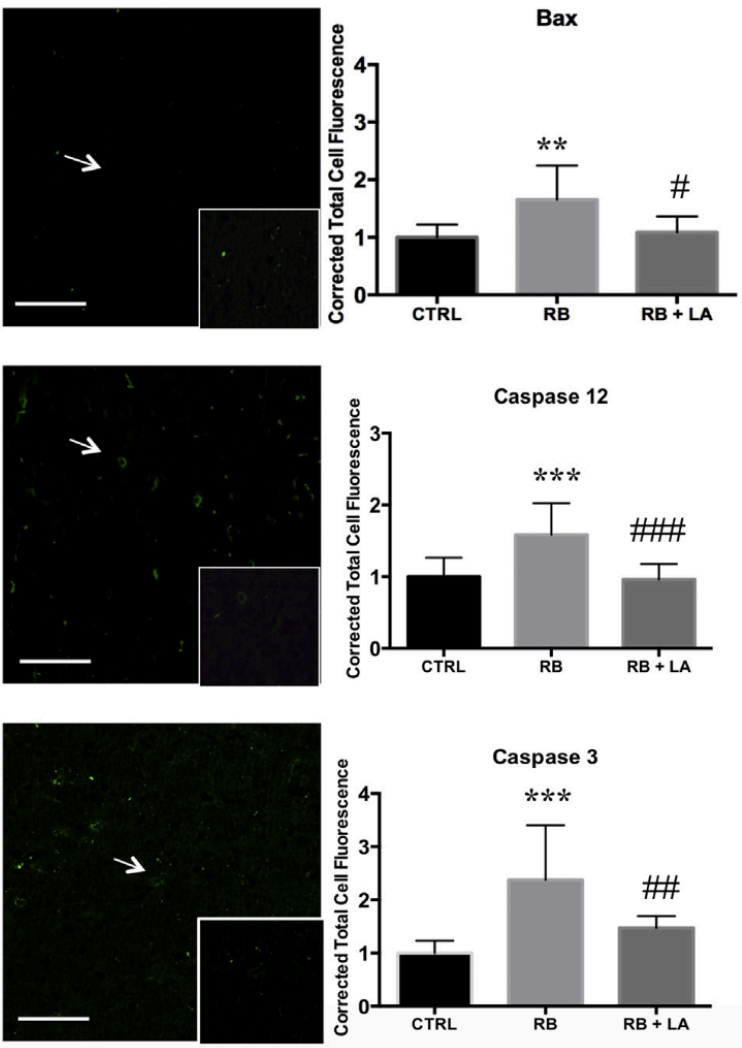

Figure 6.7: Immunohistochemistry of pro-apoptotic markers 2 weeks post repetitive blast exposure. Scale bar $=100 \mu \mathrm{m}$. (A) A significant increase in Bax expression is seen between animals exposed to repetitive blast compared to control $(\mathrm{t}=5.194, \mathrm{p}<0.01)$. Lipoic acid treatment administered 5 minutes post each blast reduced Bax expression back towards control levels compared to the repeat blast group $(t=4.483, p<0.05)$. No change is seen between the other groups. ${ }^{* *} \mathrm{p}<0.01 \mathrm{CTRL}$ vs. RB. $\# \mathrm{p}<0.05 \mathrm{RB}$ vs. $\mathrm{RB}+$ LA. (B) An increase in the expression of caspase 12 was seen for animals exposed to repetitive blast compared to control $(\mathrm{t}=5.809, \mathrm{p}<0.001)$. Alpha lipoic acid treatment reduced caspase 12 levels back towards control compared to the repeat blast group $(\mathrm{t}=6.157, \mathrm{p}<0.001)$. No change is seen between the control and the repetitive blast with alpha lipoic acid treatment groups. $* * * \mathrm{p}<0.001$ CTRL vs. RB. \#\#\# $\mathrm{p}<0.001 \mathrm{RB}$ vs. RB 
+ LA. (C) An increase is seen in the expression of caspase 3 in the repetitive blast group compared to the control group $(\mathrm{t}=6.989, \mathrm{p}<0.001)$. A significant reduction was noted when alpha lipoic acid was administered following blast compared to the repetitive blast group ( $t=5.459, \mathrm{p}<0.01)$. No change is seen between the control and repetitive blast treated with lipoic acid groups. ${ }^{* * *} \mathrm{p}<0.01$ CTRL vs. RB. \#\# $\mathrm{p}<0.01 \mathrm{RB}$ vs. RB + LA. $\mathrm{CTRL}=$ control, $\mathrm{RB}=$ repeat blast, $\mathrm{LA}=$ lipoic acid. 
Control
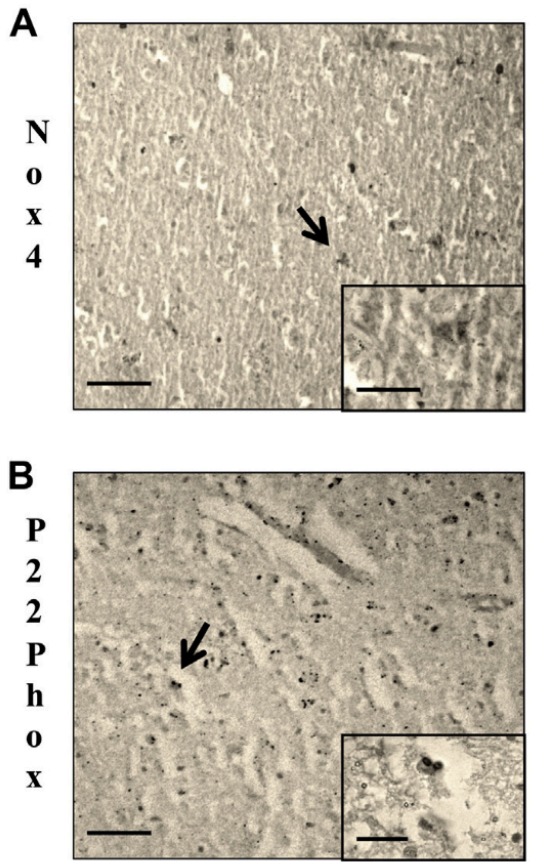

Football Player
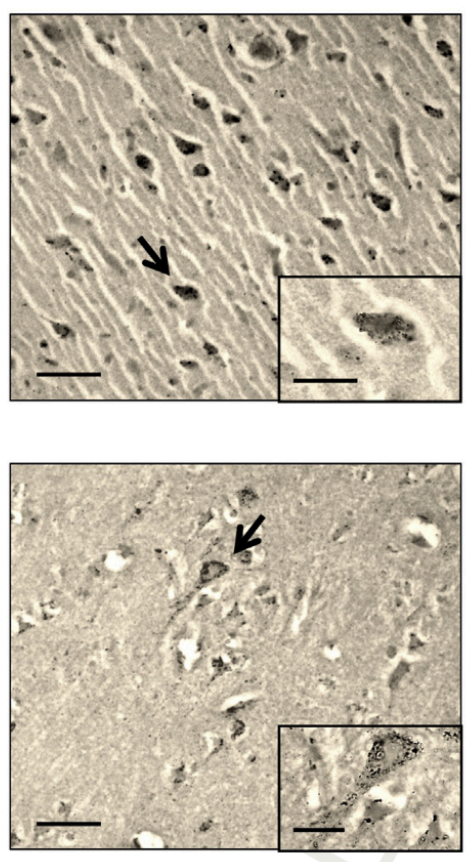

Wrestler
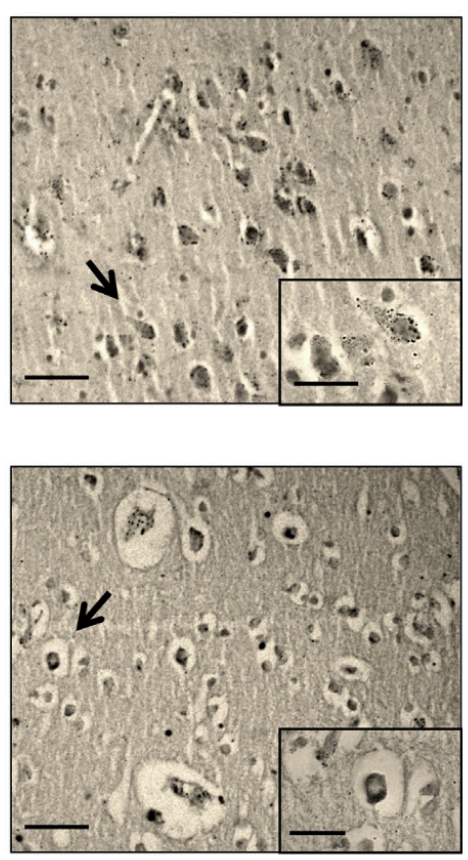

Figure 6.8: NADPH oxidase marker expression in human CTE samples. Samples obtained from the cortex of a professional football player, professional wrestler, and age and sex matched control. Scale bar for whole images $=50 \mu \mathrm{m}$. Scale bar for inlay images $=20 \mu \mathrm{m}$. (A) Nox 4 staining shows increased expression in tissue of the football player (32/100) and professional wrestler (27/100) when compared to control tissue (6/100). The increase of Nox 4 can lead to formation of reactive oxygen species that cause cellular damage. (B) P22phox forms a complex with Nox4 that leads to increased NADPH oxidase activity within the cell. An increase in the expression of $\mathrm{p} 22 \mathrm{phox}$ was seen in the samples from the football player (17/100) and wrestler (24/100) compared to control tissue $(3 / 100)$. The complex formation of these two components suggests subsequent free radical formation. 


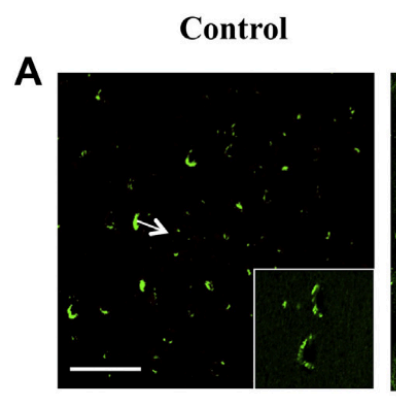

Wrestler
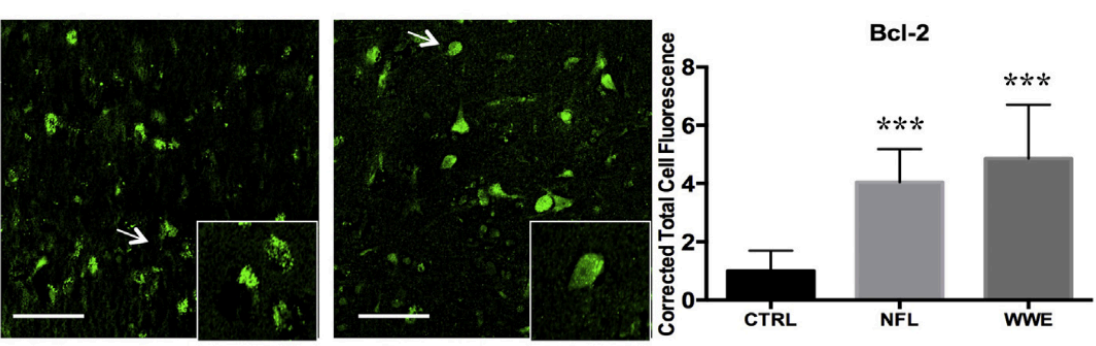

B
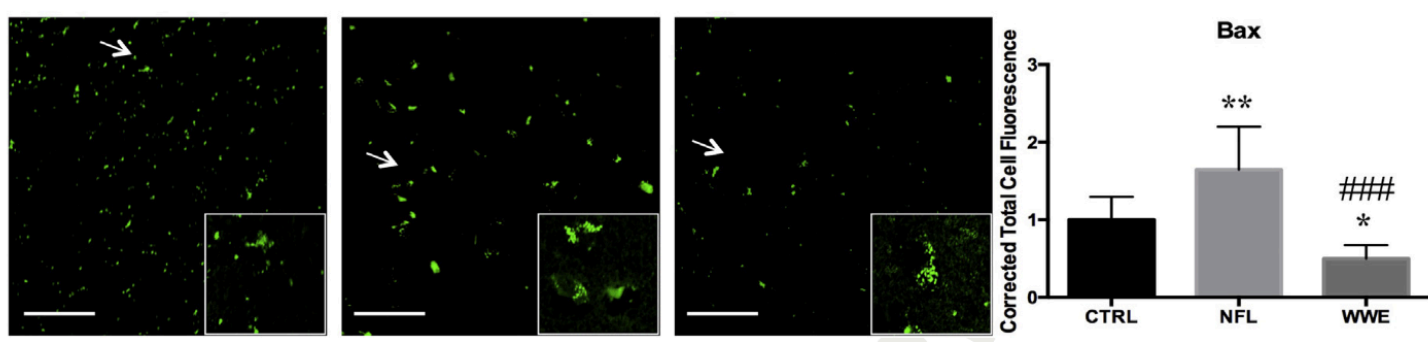

Figure 6.9: Pro-apoptotic marker expression in human CTE samples. Scale bar $=100 \mu \mathrm{m}$. (A) $\mathrm{Bcl}-2$ is a protective protein that is increased in order to prevent cellular apoptosis. In the human tissue, $\mathrm{Bcl}-2$ expression is greater in football player tissue compared to control $(\mathrm{t}=7.302, \mathrm{p}<0.001)$. Bcl-2 expression is also significantly increased between wrestler tissue compared to control $(\mathrm{t}=9.255, \mathrm{p}<0.001){ }^{* * *} \mathrm{p}<0.001 \mathrm{CTRL}$ vs. football player and CTRL vs. wrestler. The increase in Bcl-2 could be a mechanism by which neurons survive to propogate tau. (B) Bax is known to initiate the cellular apoptosis process. A significant difference was seen between the football player tissue sample and control $(\mathrm{t}=5.424, \mathrm{p}<0.01)$. A significant difference was also seen between the wrestler tissue sample and control $(\mathrm{t}=4.221, \mathrm{p}<0.05)$. A significant difference in Bax was also seen between the football player sample and wrestler sample $(\mathrm{t}=9.645, \mathrm{p}<0.001) .{ }^{* *} \mathrm{p}<0.01$ CTRL vs. football player. * $\mathrm{p}<0.05$ CTRL vs. wrestler. \#\#\# $\mathrm{p}<0.001$ football player vs. wrestler. The wrestler sample had more severe CTE pathology compared to the football player sample indicating that apoptosis may play an important role in acute disease progression but not as much of a role once it has fully developed. Tauopathy progression 
is reliant on this disrupted apoptotic signaling. $\mathrm{CTRL}=$ control, $\mathrm{NFL}=$ football player, $\mathrm{WWE}=$ wrestler. 


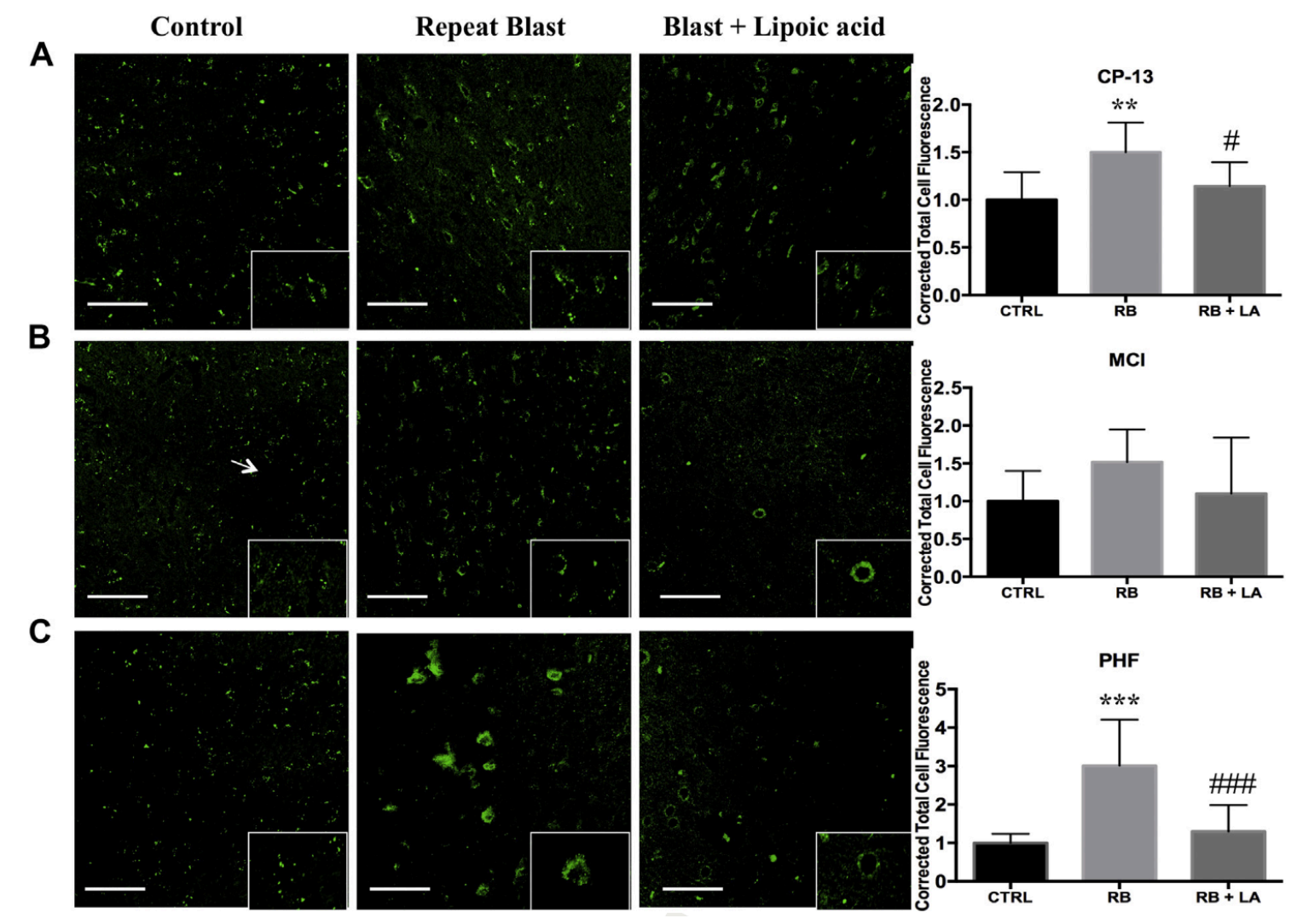

Figure 6.10: Lipoic acid reduces pathologic conformational changes in tau two weeks following repetitive blast exposure. Scale bare $=100 \mu \mathrm{m}$. CP-13 marks a form of pretangle tau and MCI is a unique conformational configuration of tau found only in neurodegenerative disease. PHF is a paired configuration of tau marking the transition towards tau accumulation in the soma. (A) a significant increase in CP-13 was noted in the prefrontal cortex following repetitive blast exposure compared to control $(q=5.54$, $\mathrm{p}<0.01)$, but the response was ameliorated by lipoic acid administration post-blasts $(\mathrm{q}=$ 3.967, $\mathrm{p}<0.05) .{ }^{* *}=\mathrm{p}<0.01 \mathrm{RB}$ vs. CTRL. $\#=\mathrm{p}<0.05 \mathrm{RB}+\mathrm{LA}$ vs. RB. (B) no significant differences between groups were observed for MCI. (C) a significant increase in PHF was noted in the prefrontal cortex following repetitive blast exposure compared to control $(q=7.865, p<0.001)$, but was ameliorated by lipoic acid administration post- 
blasts $(\mathrm{q}=6.703, \mathrm{p}<0.001) . * * *=\mathrm{p}<0.001 \mathrm{RB}$ vs. CTRL. \#\#\#=p<0.001 RB + LA vs.

RB. $\mathrm{CTRL}=$ control, $\mathrm{RB}=$ repeat blast, $\mathrm{LA}=$ lipoic acid. 


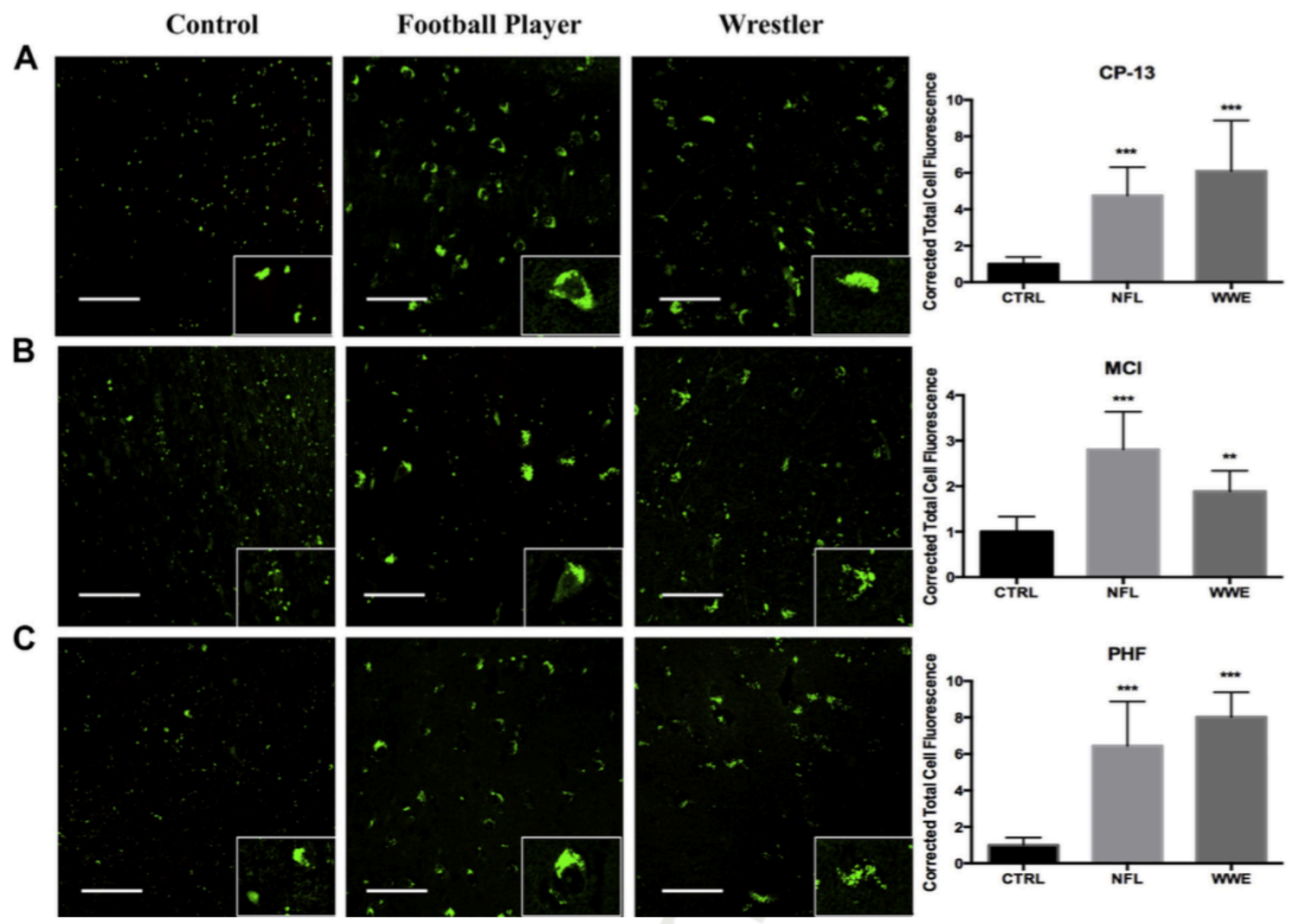

Figure 6.11: Tauopathy in human chronic traumatic encephalopathy brains. Scale bar $=$ $100 \mu \mathrm{m}$. Tauopathy is the characteristic finding of CTE. Hyperphosphorylated tau causes conformational changes, which facilitate the transition towards neurofibrillary tangles.

(A) a significant increase in CP-13 was seen between control and football player brains (q $=6.406, \mathrm{p}<0.001)$, and between control and wrestler brains $(\mathrm{q}=8.695, \mathrm{p}<0.001) . * * *=$ $\mathrm{p}<0.001$. (B) a significant increase in MCI was seen between control and football player brains $(\mathrm{q}=9.88, \mathrm{p}<0.001)$, and between control and wrestler brains $(\mathrm{q}=4.838=\mathrm{p}<0.01)$. $* * *=\mathrm{p}<0.001 . * *=\mathrm{p}<0.01$. (C) a significant increase in PHF was seen between control and football player brains $(\mathrm{q}=10.58, \mathrm{p}<0.001)$, and between control and wrestler brains $(\mathrm{q}=13.64, \mathrm{p}<0.001) . * * *=\mathrm{p}<0.001 . \mathrm{CTRL}=$ control, $\mathrm{NFL}=$ football player, WWE $=$ wrestler. 

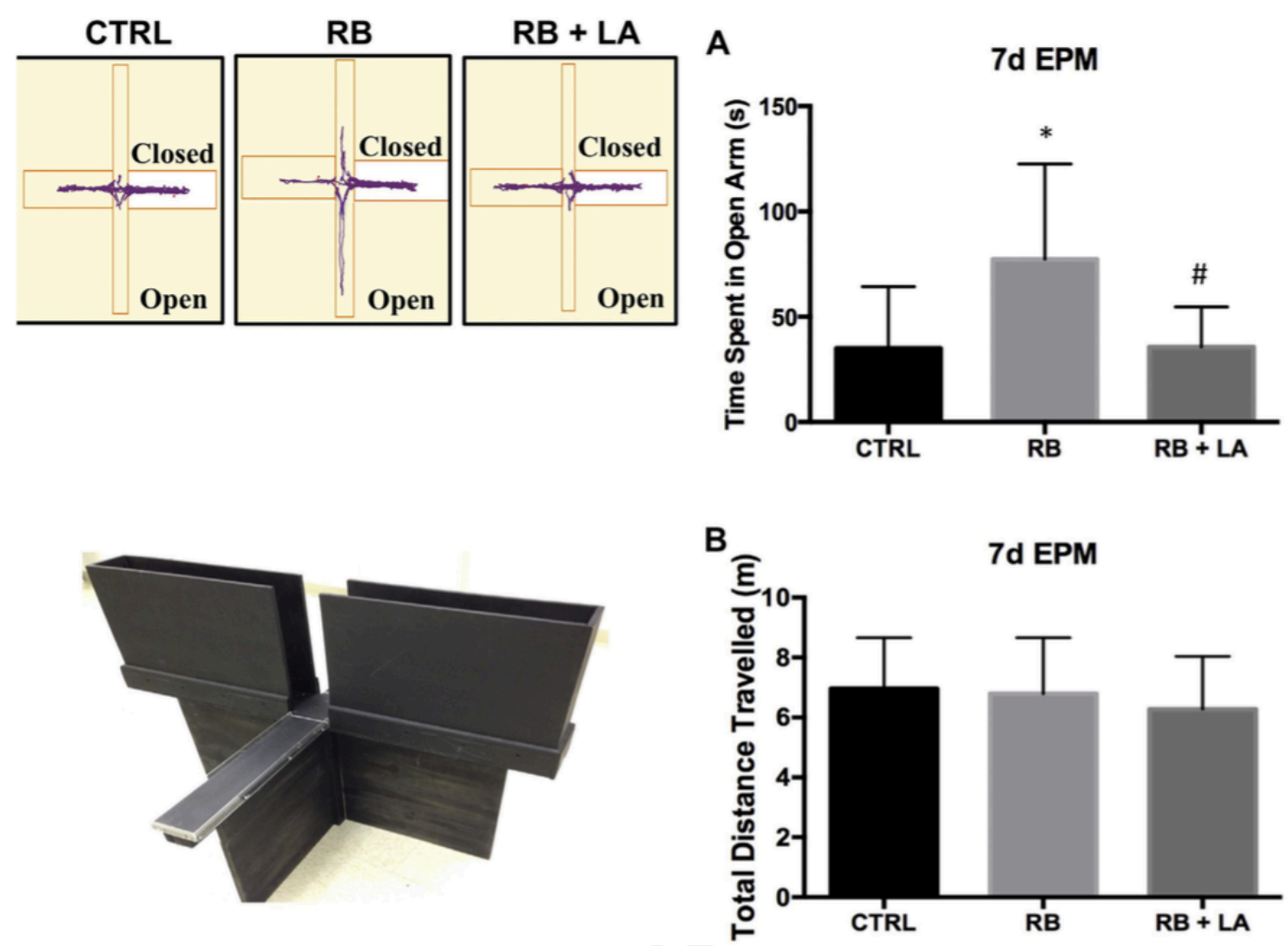

Figure 6.12: Effect of repetitive blast on impulsive-like behavior using the elevated plus maze assessment. (A) Animals exposed to repetitive blast spent more time in the open arms compared to control $(\mathrm{t}=3.632, \mathrm{p}<0.05)$. Time spent in open arm is a representation of impulsive-like behavior in rodents. Alpha lipoic acid treatment following blast decreased time spent in open arm compared to repetitive blast exposed animals $(\mathrm{t}=3.573$, p $<0.05$ ). $* \mathrm{p}<0.05$ CTRL vs. RB. \# $<<0.05$ RB vs. RB + LA. (B) The groups did not differ in terms of distance traveled in the elevated plus maze. 


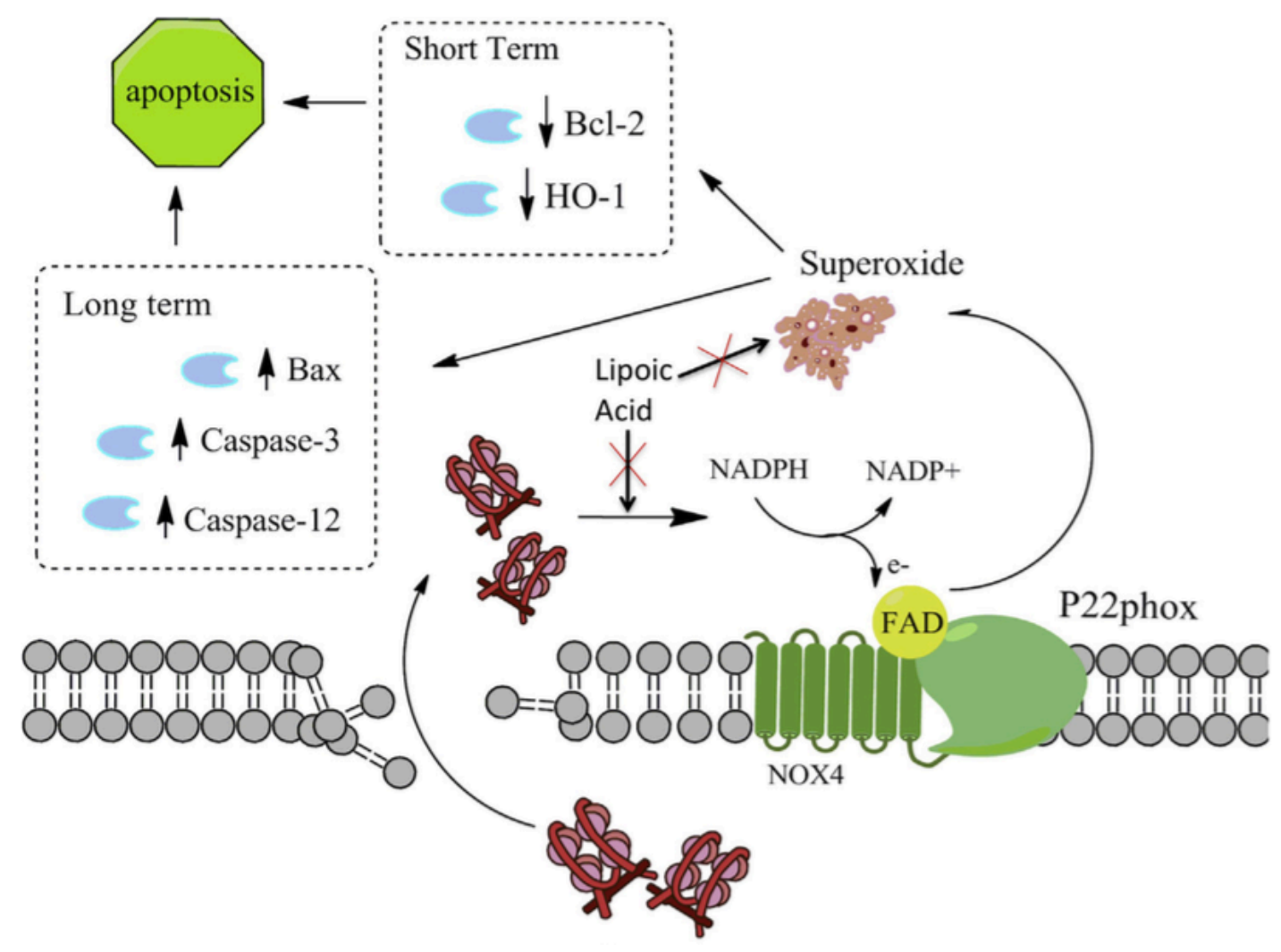

Supplementary Figure 6.1: Schematic showing Nox4 pathway post- blast. Free iron damages the intracellular membrane and leads to activation formation of the Nox 4 and P22phox complex. The Nox4 complex generates superoxide, which can decrease antiapoptotic markers acutely and increase pro-apoptotic markers subacutely. Ultimately, this pathway contributes to apoptosis and neurodegeneration. Lipoic acid has potential to target this pathway and reduce neurodegeneration following traumatic brain injury. 


\section{Chapter Seven}

Modeling chronic traumatic encephalopathy: The way forward for future discovery

This work is published in Front Neurol. 2015 Oct 26;6:223. doi:

10.3389/fneur.2015.00223. eCollection 2015. Review. PMID: 26579067

Turner RC, Lucke-Wold BP, Logsdon AF, Robson MJ, Lee JM, Bailes JE, Dashnaw ML, Huber JD, Petraglia AL, Rosen CL 


\begin{abstract}
Despite the extensive media coverage associated with the diagnosis of chronic traumatic encephalopathy (CTE), our fundamental understanding of the disease pathophysiology remains in its infancy. Only recently have scientific laboratories and personnel begun to explore CTE pathophysiology through the use of preclinical models of neurotrauma. Some studies have shown the ability to recapitulate some aspects of CTE in rodent models, through the use of various neuropathologic, biochemical, and/or behavioral assays. Many questions related to CTE development however remain unanswered. These include the role of impact severity, the time interval between impacts, the age at which impacts occur, and the total number of impacts sustained. Other important variables such as the location of impacts, character of impacts, and effect of environment/lifestyle and genetics also warrant further study. In this work we attempt to address some of these questions by exploring work previously completed using single and repetitive injury paradigms. Despite some models producing some deficits similar to CTE symptoms, it is clear that further studies are required to understand the development of neuropathological and neurobehavioral features consistent with CTE-like features in rodents. Specifically, acute and chronic studies are needed that characterize the development of tau-based pathology.
\end{abstract}

KEY WORDS: Chronic traumatic encephalopathy, preclinical models, neurodegeneration, hyperphosphorylated tau, neurotrauma 


\section{INTRODUCTION}

Corsellis described the original case series of chronic traumatic encephalopathy (CTE) in boxers (Corsellis et al 1973). The disease consisted of brain atrophy, dilated ventricles, a cavum septum pellucidum, and pallor of the substantia nigra. Chronic traumatic encephalopathy was reintroduced into the medical lexicon by Dr. Bennet Omalu in 2005 (Omalu et al 2005). Omalu described a progressive tauopathy that was seen in the brains of deceased football players. In recent years, McKee, Goldstein, and Stern have defined clinical and pathologic features of the disease. These include behavioral disturbances such as impulsivity, depression, and lack of oversight (Corsellis et al 1973, Mez et al 2013). Pathologic criteria include neurofibrillary tangles in a perivascular distribution and within superficial cortical areas with occasional amyloid and TDP-43 protein aggregations (Baugh et al 2014). Stern recently expanded the CTE criteria further by describing young vs. old onset based on symptom manifestation (Stern et al 2013). Interestingly, blast traumatic brain injury has been linked to CTE following a single exposure, where athletes develop the disease following repetitive head injury (Lucke-Wold et al 2014c).

CTE has been defined as a slowly progressive disease that takes years to decades to develop, often providing a significant latent period between when the neurotrauma occurs and when symptoms develop. A few cases involve athletes/soldiers as early as late teen's to early twenties (Omalu et al 2011a, Tartaglia et al 2014). The reason for the discrepancies in age of presentation observed is currently unknown but is likely due to the age at which impacts were sustained and the severity of the injury. Prior studies have shown that children with TBI have inadequate development of social cognition (Ryan et 
al 2014) and that adolescents can develop post-traumatic headaches. Very rarely however do either of these groups experience symptoms of CTE (Dubrovsky et al 2014). Early behavioral symptoms of CTE usually do not appear until the mid-thirties, and cognitive impairment does not begin until the early sixties (Baugh et al 2014). Recent evidence suggests that neurotrauma may be linked to other neurodegenerative diseases such as Alzheimer's disease (AD) as well (Johnson et al 2010b).

$\mathrm{CTE}$ and $\mathrm{AD}$, despite both being tauopathies, have generally been viewed as separate diseases. Each disease has distinct clinical presentations and unique clinical risk values. This view has gradually been changing with some reports showing that CTE can develop within the context of $\mathrm{AD}$. The relationship between these two conditions however is poorly understood. Prior studies have indicated that TBI is a risk factor for development of cognitive impairment and AD (Guskiewicz et al 2005), but whether the conditions are additive or synergistic remains unclear (Weber 2007). Recent evidence suggests that neurotrauma may both increase the likelihood of disease development and accelerate the development of $\mathrm{AD}$ (Barnes et al 2014). Notably, the wealth of $\mathrm{AD}$ pathology observed in preclinical neurotrauma models supports the idea of disease acceleration (Tran et al 2011). In addition to AD, some groups have presented a possible link between CTE and a variant of amyotrophic lateral sclerosis that has been termed 'chronic traumatic encephalomyelopathy' (CTEM) (McKee et al 2010). Therefore, it is clear that neurotrauma may have many lasting deleterious consequences, including the potential for increased risk and accelerated development of neurodegenerative diseases such as $\mathrm{AD}, \mathrm{CTE}$, and CTEM. While the data on these studies is preliminary in nature 
and not prospective, these findings demonstrate the need for further investigation with both preclinical models and clinical trials.

\section{THE QUEST FOR THE IDEAL CTE MODEL}

The search for ideal preclinical models to study CTE remains an area of ongoing investigation with a relative paucity of prior studies. Of those published previously, the majority of studies fail to recapitulate the extensive neuropathologic and neurobehavioral aspects of injury (Goldstein et al 2012, Ojo et al 2015). Post-mortem identification of neurofibrillary tangles are a key diagnostic marker used clinically (Baugh et al 2012). Therefore, the development of hyperphosphorylated tau in animals following injury must be an important component in establishing a CTE model. Tau hyperphosphorylation appears to correlate with the emergence of neuropathologic and neuropsychiatric deficits representative of neurotrauma related neurodegeneration (Petraglia et al 2014c). By modeling CTE in rodents, we can better understand disease development and discover potential therapeutic avenues. Similarly, little justification is given to injury severity, number of impacts, interval between impacts, and age at which the impacts occur. Few studies have also evaluated secondary mechanisms of injury. In the following sections, we discuss the advancements and shortcomings of prior research while highlighting areas in need of further investigation.

\section{Proper controls to use when studying CTE}

One of the challenges in creating a preclinical model for CTE is the establishment of proper controls comparing sham to TBI animals (Weber 2007). Specifically, sham animals must undergo the exact same procedure each day of the injury paradigm. They 
must be anesthetized for the same duration as injured animals. The age of the animal must also be considered. Control animals should be the same age as experimental animals at time of sacrifice. This is an important consideration in designing extended studies with behavioral assays. Biochemical time courses also require control animals for each time point. Numerous questions must be addressed in planning experiments while considering the implications of each decision in terms of experimental question addressed and potential complications (Figure 7.1).

\section{Available tools to study CTE}

One of the current weaknesses of clinical investigation of CTE is that the disease remains a neuropathological diagnosis. Some advancement has been made in ligandbased PET imaging. The imaging was used in a cohort of patients with an extensive history of neurotrauma, but the modality is not readily available (Small et al 2013). Consequently, clinical observations are largely retrospective in nature and it is nearly impossible to investigate disease onset and progression. These clinical shortcomings can be readily addressed through the use of adequately designed preclinical models.

The first step in creating a preclinical model of CTE is to choose a model that can be used to generate a combination of biochemical and behavioral changes post-injury consistent with the CTE-like phenotype. Specifically, tauopathy or a precursor of tauopathy (tau hyperphosphorylation), must be present. The changes in tau must be inducible in genetically unaltered animals and then must be verified with transgenic rodents. Behavioral changes must be induced by neurotrauma. These changes should persist or worsen at chronic time points post-injury. Assessment of behavior should 
include tests capable of evaluating behavioral symptoms reported in clinical CTE cases. In particular, tests for cognition, depressive-like behavior, and impulsive-like behavior should be used. Cognition can be measured using Morris water maze (MWM) whereas depressive-like behavior can be assessed with the forced swim test or tail suspension test. Impulsivity can be elucidated with the elevated-plus maze (EPM).

Once a model is established, biochemical, electrophysiological, and advanced imaging techniques can be employed to assess neural injury. Biochemical mechanisms of potential interest include those related to cell survival/death, regulation of tau phosphorylation/dephosphorylation (kinases/phosphatases), bioenergetics, and propagation of tau-based changes. Electrophysiological studies can be performed to identify effects of injury and CTE development both on individual synapses and larger tracts such as the Schaffer collaterals within the hippocampus. Imaging modalities such as magnetic resonance imaging (MRI), functional MRI (fMRI), spectroscopy (MRS), and PET studies have the unique advantage of being able to be performed longitudinally with multiple assessments of the same animal at different time points. Taken together, integration of biochemical, behavioral, electrophysiological, and imaging modalities may provide insight into the mechanisms and time course of CTE development. This approach will also allow for more definitive evidence to be gathered. This evidence will provide a stepping-stone in addressing key questions about the effect of inter-injury interval, injury severity, and number of cumulative injuries necessary for the development of CTE. The answers garnered from this potential work may then influence the design of clinical trials that dictate return-to-play decisions. Baseline monitoring may become required in sports arenas and battlefields with high incidences of neurotrauma. 
The monitoring may assist in detecting cumulative subthreshold injury levels and be used to decrease the overall level of concussions.

\section{IMPLICATIONS FOR MODELING CTE: RESULTS FROM PRIOR CTE MODELING STUDIES}

Perhaps one of the most promising studies, with regards to demonstrating CTElike disease in a rodent model, was performed by Luo, et al. In this study, the authors developed a closed head model of neurotrauma utilizing an electromagnetic stereotaxic impact device. The authors showed that enhanced force of injury or using repeat injury increased GFAP-tagged luciferase. Intriguingly, when this same repeat injury paradigm was applied to wild-type mice, spatial learning and memory deficits were observed 2 to 6 months after injury and were accompanied by increased hyperphosphorylated tau and astrogliosis (Luo et al 2014a). The gliosis response in human CTE has not been well characterized, but the findings by Luo and colleagues show that GFAP was increased near areas of tauopathy. Similar findings have been seen by Petraglia and colleagues who performed a rigorous investigation of the behavioral effects of both single and repetitive closed head injury in wild-type mice (Petraglia et al 2014b). The authors found that a single injury, in un-anesthetized animals, produced notable short-term abnormalities in behavior similar to a post-concussive state. Repetitive injury (42 impacts total: 6 impacts per day over 7 days) produced chronic deficits, particularly with regards to depressive-like and risk-taking behaviors as well as spatial learning and memory (Petraglia et al 2014b). This same group published a recent follow-up study demonstrating the presence of hyperphosphorylated tau, a precursor of neurofibrillary tangle (NFT) development, in repetitively injured animals (Petraglia et al 2014c). The 
model produces several of the same behavioral symptoms reported by patients suspected of having CTE. We recently show that endoplasmic reticulum stress contributes to tauopathy and CTE-like behavioral deficits following repeat blast injury (Lucke-Wold et al 2015d).

Other studies, conducted by Mouzon and colleagues report both behavioral and neuropathologic changes following repetitive neurotrauma in mice. Specifically, 5 injuries administered 48 hours apart, produced durable cognitive deficits, learning disabilities, diminished rotarod performance, and changes in anxiety-like behavior on elevated-plus maze. Notably, these behavioral changes occurred in conjunction with persistent neuroinflammatory changes and disruption of white matter integrity. No changes in $A \beta$ and tau phosphorylation were seen at the chronic time points of 6 and 12 months post-neurotrauma, likely because rodents don't naturally develop neurofibrillary tangles (Mouzon et al 2014). Rodents do however demonstrate acute tau changes due to phosphorylation and cleavage following injury (Huber et al 2013). Liu and colleagues found that tau hyperphosphorylation was increased in rats acutely post-injury and triggers caspase activation in rat cortices (Liu et al 2011). The activation of cell death can lead to circuit dysfunction and behavioral deficits (Abisambra \& Scheff 2014). Goldstein and colleagues show that tauopathy contributes to mitochondrial dysfunction and microtubule injury that ultimately leads to apoptosis. In this study, they found that tau modulation is a potential avenue for therapeutic intervention (Kondo et al 2015). We recently report that caspase activation was increased in human CTE brains near sites of neurofibrillary tangle formation (Lucke-Wold et al 2015a). 
Other repeat-injury studies have investigated changes in tau and amyloid postinjury with the goal being to more clearly elucidate the relationship between TBI and neurotrauma related neurodegeneration. One report showed an increase in neuronal tau immunoreactivity (Kanayama et al 1996) and another showed elevated amyloid precursor protein (APP) (Dobrowolska \& Gibson 2005, Weber 2007) at a variety of time points post-injury. A final study by Zhang and colleagues showed that monoacylglycerol lipase can lead to behavioral deficits and tauopathy characteristic of a CTE-like phenotype (Zhang et al 2015). These findings were further verified in other studies using transgenic models of amyloidosis and tauopathy in which repetitive injury paradigms produced elevated amyloid and tau levels with increased deposition. Single injury however failed to produce changes above control levels (Conte et al 2004, Uryu et al 2002). Using a T44 tau Tg mouse line, Yoshiyama and colleagues sought to study dementia pugilistica (DP), a condition sharing many features with modern day CTE, but with several key differences. DP by definition occurs only in boxers and has more severe gross anatomical changes that are not always present in CTE. The Yoshiyama group found that 4 impacts per day given once a week for 4 weeks produced only modest neuropathology with only 1 mouse demonstrating CTE or DP characteristics out of a total of $18 \mathrm{Tg}$ mice and 24 wild-type mice (Weber 2007, Yoshiyama et al 2005). The affected mouse displayed neuropathologic changes that included heightened tau burden, the presence of neurofibrillary tangles, and cognitive deficits (Yoshiyama et al 2005). While it remains unclear why only one mouse developed such pathology, neuropathological findings from this one animal demonstrated that iron deposition was increased and associated with blood-brain barrier disruption. Iron deposition, associated with degradation of heme, 
activates oxidative stress-related pathways. Importantly, this oxidative stress is then associated with accelerated neurofibrillary tangle formation in these perivascular locales (Yoshiyama et al 2005).

Notably, even single injury paradigms have been shown to produce tauopathy in a variety of injury models including blast (Goldstein et al 2012), fluid percussion (Hawkins et al 2013), and controlled cortical impact, findings consistent with prior clinical reports (Begum et al 2014, Rubenstein et al 2014). Goldstein and colleagues demonstrated that tauopathy following single blast injury was associated with hippocampal-dependent learning and memory deficits at subacute and chronic time points. These changes were also associated with electrophysiological alterations in long-term potentiation (Goldstein et al 2012). Therefore, even a single mild to moderate injury may induce neurodegeneration and neurological deficits leading to impaired cognition and disrupted synaptic transmission (Goldstein et al 2012). Single injury has also been shown to contribute to blood brain barrier disruption (Glushakova et al 2014). This is a significant finding considering the numerous concussive and subconcussive injuries occurring in athletics and on the battlefield. Other studies using single injury models have also demonstrated activation of numerous pathological processes and behavioral changes associated with neurodegeneration. Modeling neurotrauma related neurodegeneration is a key component in the search for a model of CTE (Logsdon et al 2014, Lucke-Wold et al 2014a, Smith et al 1995). While the importance of repeat injury in CTE modeling cannot be overstated, some single injury studies have led to advances in the ability to detect phospho-tau in serum at weeks to months post-injury. These advances indicate the potential role of biomarkers in monitoring and understanding disease pathophysiology 
(Rubenstein et al 2014). The development of animal based models that exhibit similar characteristics of CTE will afford researchers the opportunity to characterize the acute and chronic effects of injury on the phosphorylation of tau in controlled experimental conditions. In vitro models may even be beneficial in elucidating changes at the cellular level (Zander et al 2015). Evaluating imaging modalities, potential biomarkers, such as phosphorylated tau, and proposed therapeutics in a controlled context will promote advancement towards clinical applications and could be instrumental for monitoring and understanding disease pathophysiology in the future.

\section{IMPLICATIONS FOR MODELING CTE: LEARNING FROM PAST SHORTCOMINGS WITH TBI MODELS}

Despite these notable advancements in CTE modeling as outlined above, the vast majority of repeat injury studies fail to address the role of injury severity, inter-injury interval, and the total number of impacts needed to reproduce a CTE-like state (Goddeyne et al 2015, Prins et al 2010). Most studies simply describe features of TBI without relating the findings to neurodegeneration (Loane \& Kumar 2015). Furthermore, because CTE is a neuropathologic diagnosis, any study claiming to serve as a model of CTE must demonstrate the hallmark neuropathologic changes (tau hyperphosphorylation and neurofibrillary tangles) and show that these changes persist at delayed time points and coexist with behavioral deficits. Few studies have looked at amyloid, tau, or TDP43 accumulation following injury.

One of the most common omissions from repetitive injury paradigms is the lack of consideration for inter-injury interval. Work by Longhi and colleagues directly 
explored this issue, demonstrating that in a mouse model of closed-head injury (CHI), mice had a period of vulnerability estimated between 3 and 5 days where the effect of injuries was additive and produced deficits in cognition. When the inter-injury interval was lengthened to 7 days, these deficits were not present (Longhi et al 2005). While the focus of that study was elucidating differences in the inter-injury interval, other groups employing a repeat injury paradigm should consider how the inter-injury interval may relate to their findings. Inter-interval design will be an important consideration in developing CTE models. Attempting to provide a clinical context for these decisions would be desirable, with the interval between severe concussive-type impacts ideally being longer than that between mild subconcussive-type impacts. This would expand scientific insight for "return-to-play" guidelines that require players to be asymptomatic following a diagnosed concussion prior to returning to contact sports. These types of questions have generally not been considered in preclinical work, with most studies using brief inter-injury intervals, often ranging from minutes to 24 hours with poor justification for timing (Creeley et al 2004, Genovese et al 2013, Klemenhagen et al 2013, Petraglia et al 2014b, Shitaka et al 2011, Wang et al 2014).

Another important limitation related to interpretation of injury severity and neuropathologic outcome, is the sensitivity of detecting neuropathology using current scientific approaches. Shitaka and colleagues demonstrated previously that silver staining was more reliable for detecting axonal injury and pathology in comparison to routine histological analysis, assessment of neuronal cell loss, and amyloid precursor protein (APP) immunohistochemistry (Shitaka et al 2011). It may be increasingly important, particularly in studies of repetitive subconcussive impacts, to utilize measures 
of high sensitivity for injury detection. Silver staining, which has been shown repeatedly to exhibit a higher degree of sensitivity in detecting axonal injury than many immunohistochemical techniques; electron microscopy; or other markers with these characteristics may prove promising (Shitaka et al 2011). It has not yet been determined if neurofibrillary tangles accumulate around sites of axonal shearing.

\section{KEY QUESTIONS GOING FORWARD}

Improving experimental models will enhance the quest for developing therapeutic agents that can be used to prevent and treat CTE. The search for a model of CTE raises a number of questions that are important clinically. These questions include issues such as length of the inter-injury interval, the number and severity of impacts, and the age at time of impacts, as well as the mechanism of impact, the gender of the patient, and what role genetic predisposition may play in the development of neurodegenerative disease following neurotrauma. Another important question that must be addressed is does a history of neurotrauma and potential presence of CTE accelerate the development of Alzheimer's disease? Furthermore, how can the period of susceptibility following neurotrauma be identified most readily? In the following sections we attempt to address these questions based upon available evidence. We also provide suggestions for handling shortcomings going forward. Studies upon which these sections are based are referenced in Tables 7.1-7 for quick reference.

\section{Inter-injury interval}

The effect of inter-injury interval on outcome following TBI has only recently been investigated. Studies have explored a variety of intervals ranging from 2 minutes apart 
(in vitro) to a few hours (in vivo) to as long as 30 days apart (in vivo). Using a novel approach assessing vasoreactivity in TBI, Fujita and colleagues demonstrated that administration of seemingly mild injuries at brief intervals ( 3 hours apart) produced dramatic declines in vasoreactivity and axonal pathology. When the inter-injury interval was lengthened to 5 hours, the magnitude of these changes was diminished substantially with complete dissolution of changes in both pathology and vasoreactivity at 10 hours (Fujita et al 2012).

Other studies that have investigated longer inter-injury intervals have identified periods of susceptibility following an initial impact at periods ranging from 24 hours to a few weeks (Bolton \& Saatman 2014, Mannix et al 2013, Meehan et al 2012, Weil et al 2014). Bolton and colleagues demonstrated with a CHI model that a single impact produced extensive gliosis bilaterally in the hippocampi and entorhinal cortices. Repeat injury after 24 hours produced a more severe injury consisting of hemorrhage in the entorhinal cortices as well as heightened measures of neurodegeneration, gliosis, and neuroinflammation (Bolton \& Saatman 2014). When the experimental paradigm was changed such that impacts were given with a 48-hour inter-injury interval, the histopathology resembled that of a single impact suggesting enhanced susceptibility when a second impact was administered within 24 hours (Bolton \& Saatman 2014).

Mannix and colleagues performed one of the most rigorous investigations of the effect of inter-injury interval on outcomes associated with TBI. These studies included measures of both cognition and neuropathology related to the development of neurodegenerative diseases such as CTE and AD. In this study, the investigators showed that animals who received daily or weekly injuries with weight-drop had persistent 
cognitive deficits up to 1 year post-injury (Mannix et al 2013). This was in contrast to when animals were injured biweekly or monthly, which failed to produce deficits at such a chronic time point (Mannix et al 2013). Notably, the cognitive deficits seen in the daily- and weekly-injured animals did not correlate with elevations in tau phosphorylation or amyloid- $\beta$ when measured by ELISA nor brain volume loss when measured by MRI (Mannix et al 2013). This finding may indicate that in addition to inter-interval time, injury severity must be considered. Tauopathy is essential for modeling CTE therefore an appropriate inter-injury interval might be best characterized in transgenic animals. Weight drop produces variable injury based on the height of the drop. A more severe TBI can produce cognitive deficits but may not be representative of the concussive and subconcussive injuries associated with CTE. A mild TBI with a transgenic animal will likely produce the most relevant deficits.

Meehan and colleagues performed a similar study with primarily behavioral assays. The investigators subjected mice to a $\mathrm{CHI}$ via weight-drop for a total of 5 impacts at various intervals. These intervals included daily, weekly, and monthly intervals. Mice receiving 5 impacts total at daily or weekly intervals were impaired in the MWM compared to sham animals (Meehan et al 2012). This was not the case when injuries were delivered at monthly intervals, as these animals exhibited no impairment in the MWM (Meehan et al 2012). Interestingly, at one month post-injury, the daily- or weekly-injured animals still exhibited deficits in the MWM and this deficit persisted in daily-injured animals out to 1 year (Meehan et al 2012). This finding may represent why football lineman who experience daily subconcussive injuries appear more likely to develop CTE based on the clinical cases reported. It is still necessary to establish if 
tauopathy is the driving mechanism behind behavior. The findings confirm and expand upon the inter-interval studies completed by Longhi and colleagues (Longhi et al 2005). Longhi reported that shorter inter-interval injuries produce worse outcomes, which is in agreement with the Mannix and Meehan findings. These studies were consistent with findings in higher phylogenetic species as well, specifically piglets. Friess and colleagues showed that a 24-hour inter-injury interval produced more severe deficits and higher mortality rates than when the interval was extended to 7 days (Friess et al 2009). Finally, Kanayama and colleagues demonstrated a graded response in locomotor activity. Both shorter inter-injury intervals as well as greater number of total injuries were associated with worse outcome (Kanayama et al 1996).

Weil and colleagues explored the effect of altering the inter-injury interval in relation to recovery from TBI. They used a clear clinical-minded approach and utilization of metabolic imaging (PET). This group showed that injuries separated by only 3 days were associated with worse neuropathology and an inability to mount the typical hypermetabolic response with regards to glucose utilization following TBI. This worse outcome was not seen following either a single injury or repeat injuries with an extended inter-injury interval of 20 days (Weil et al 2014). Similarly, a brief inter-injury interval of 3 days was associated with elevated IL- $1 \beta$ and TNF $\alpha$ gene expression when compared with other experimental groups (Weil et al 2014).

The longest interval between injuries used in preclinical studies, to the best knowledge of the authors, was 30 days. The additional injury had no additive effect on anxiety (EPM), depression (FST), and cognitive function (MWM) when compared to animals receiving only one injury (Mychasiuk et al 2014). These findings indicate that 
either the window of vulnerability following the first injury was avoided or that the response to the first injury may protect the animal from subsequent injuries, a concept known as preconditioning (Mychasiuk et al 2014). The progression towards tauopathy was not well characterized in this work.

In vitro studies have shown similar findings to the in vivo studies described above. Shorter inter-injury intervals between mechanical stretching resulted in an elevation in S$100 \beta$ protein release and increased cellular permeability identified with propidium iodide staining (Weber 2007). Similarly, a 'subthreshold' level of stretch did not produce any overt cellular damage or death when repeated at 1-hour intervals but did cause neuronal loss and neuron-specific enolase (NSE) release when performed at incredibly short intervals (every 2 minutes) (Slemmer \& Weber 2005, Weber 2007). Remarkably, this rapid and repetitive 'subthreshold' stretch that produced changes in neurons, failed to produce an increase in S-100 $\beta$ protein release, indicating a differential response between neurons and glia to neurotrauma severity and interval (Slemmer \& Weber 2005, Weber 2007).

In contrast to the above studies, one group showed that repeat injury, when administered in different anatomical locations within the brain, failed to result in heightened damage when an inter-injury interval was 3 days. It did however increase tissue vulnerability with a 7-day interval as evident by increased hemorrhage (Donovan et al 2012, Donovan et al 2014). The authors of the study therefore argue that the period of susceptibility likely depends on not only the time interval between injuries, but also the anatomic location of injury (Donovan et al 2012, Donovan et al 2014). Importantly, this 
study utilized an open injury model (controlled cortical impact), a scenario that is only seen in a subset of clinical neurotraumas.

In summary, these studies demonstrate a period of vulnerability following initial injury in which sustaining a second brain injury may result in an additive effect. Additive injury is not clearly apparent when the brain is allowed a more extensive recovery period. Interestingly, this vulnerability may not solely be due to initial axonal pathology but may also be the result of cerebrovascular reactivity and the inability to utilize glucose effectively (Fujita et al 2012, Weil et al 2014). This concept of a varied cellular response to $\mathrm{TBI}$ is consistent with findings from in vitro studies that demonstrate a varied response amongst glia and neurons (Slemmer \& Weber 2005, Slemmer et al 2004, Weber 2007).

\section{Number of impacts}

The prevailing theory for 'mild' neurotrauma is that repetitive injuries are associated with more short- and long-term detrimental effects than a single injury alone (Bolton \& Saatman 2014, Laurer et al 2001, Mouzon et al 2012). This concept has been applied regardless of injury severity (concussive versus subconcussive) with emerging evidence indicating that even subthreshold impacts are cumulatively detrimental (Bailes et al 2013, Fujita et al 2012). In this section we explore the effect of repeat injury through analysis of studies employing both repeat and single injury paradigms.

One of these studies, conducted by Mouzon and colleagues, showed that when rodents are exposed to 5 total injuries with an interval of 48 hours, these animals exhibit both impaired learning and memory at extended time points (Mouzon et al 2014). These findings are in contrast to single injured animals that display only learning deficits but no 
retention impairment at the same time points (Mouzon et al 2014). This study closely parallels the clinical findings documented by Guskiewicz and colleagues in which former athletes with a history of repetitive concussions experience memory-related issues at a rate 5 times higher than those without a history of concussion (Guskiewicz et al 2005, Mouzon et al 2014).

Other work that investigated various iterations of impacts $(0,1,3,5$, and 10$)$ demonstrated that while a single injury does not produce deficits in MWM performance in comparison to sham-injured animals, repetitive injury does in fact produce deficits and these deficits may exhibit a dose-dependent relationship. When mice were given 10 concussive weight-drop injuries, those in which the weight was dropped from a height of 42 inches performed worse than those injured from a height of 38 inches. Therefore, this work demonstrates the potential for both injury number and injury severity in contributing to neurological dysfunction (Meehan et al 2012). Luo and colleagues utilized a GFAP-driven luciferase mouse line and a repetitive closed head injury model to investigate cumulative decline. The investigators showed that there appeared to be a linear increase in GFAP luminescence from 1 to 3 injuries but that this response appeared to reach a plateau by 5 injuries (Luo et al 2014a). In addition to the increase in GFAP fluorescence with repetitive injuries, mice receiving three injuries demonstrated less freezing time than sham animals in both cued and contextual fear conditioning (Luo et al 2014a). This was in contrast to single injury animals that did not differ from shaminjured animals in cued or contextual memory (Luo et al 2014a). Consequently, this data demonstrates that an increase in injury number is associated with an increasing severity of injury markers (based on protein expression of GFAP) as well as functional deficits 
(fear conditioning). Others employing both single and repeat injury paradigms have shown that while single injury may not induce pathological findings, repeat injury, of the same severity, does. This was particularly notable in work by Uryu and colleagues in which repetitive injury produced an increase in $\mathrm{A} \beta$ deposition in $\mathrm{Tg} 2576$ animals, whereas no increase was observed in single injury paradigms in these same animals (Uryu et al 2002). Likewise, Kanayama and colleagues demonstrated that repeat injury paradigms induced tau hyperphosphorylation, a precursor to NFT formation, in conditions such as CTE and AD (Kanayama et al 1996).

The observed findings in closed-head, repeat injury models, were also consistent with those found in open-head injury using a craniectomy and controlled cortical impact. In work by Donovan and colleagues, the investigators showed that repeat injury induces progressive and evolving changes that are not observed in single injury paradigms (Donovan et al 2014). The basis of many of the memory and cognitive changes following repeat TBI, may be explained by electrophysiological alterations in synaptic transmission. In work by Aungst and colleagues, repetitive TBI was found to prevent the induction of long-term potentiation (LTP) 28-days post-injury due to alterations in the NMDA receptor. This is in stark contrast to single injury paradigms that revealed the ability to induce LTP in both hemispheres, with the contralateral hemisphere exhibiting less LTP than the ipsilateral hemisphere. The impairments in excitatory neurotransmission following repeat injury were accompanied by extensive neuroinflammation and neurodegeneration as well as behavioral/functional impairments (Aungst et al 2014b). Specifically, repeat injury produced deficits more severe than single injury when measured at one-week intervals out to a month. Importantly, even 
single injury produced deficits at chronic time points post-injury when compared to sham animals, indicating long-term effects of TBI (Aungst et al 2014b).

Povlishock and colleagues expanded the concept of subthreshold injury. In these studies, the investigators showed that administering a single weight-drop injury from 1.0 $\mathrm{m}$ resulted in neither axonal nor microvascular change. With repeat injury of short interinjury intervals (hours), significant axonal and microvascular pathology was observed (Fujita et al 2012). This work was the first to assess microvascular reactivity to acetylcholine (ACh) following repetitive subthreshold brain injury. This work demonstrates the clear danger of subthreshold impacts when sustained in a repeated and rapid fashion. It also illustrates the role of the microvasculature in neuronal injury, showing that the neurovascular unit is essential for neuronal homeostasis.

Importantly, work regarding the number of impacts has been extended higher up the phylogenetic tree to rabbits and piglets (Olsson et al 1971, Raghupathi et al 2004). In rabbits, repeated loading with loads of up to 1.5 atm failed to produce an additive concussive response over a single load. A multi-loading paradigm at higher loads however caused respiratory arrest (Olsson et al 1971). In piglets, multiple less severe injuries induced neuropathological findings similar to a severe load based on the density of injured axons as well as number and distribution of foci (Raghupathi et al 2004).

In vitro studies have reported similar findings to the in vivo studies. Weber and colleagues applied a mild stretch to hippocampal neuronal cultures that produced lowgrade injury when applied at a single time. When this injury was repeated, the cells 
exhibited cumulative damage with two injuries inducing an increase in neuron-specific enolase (NSE) (Slemmer \& Weber 2005, Slemmer et al 2004, Weber 2007).

Notably, the group led by Mychasiuk and colleagues provides evidence contrary to the widely held belief that an increased number of impacts are associated with detrimental findings on behavioral or histological measures. With a 30-day inter-injury interval, rodents receiving multiple injuries performed similar to single-injury animals on measures of anxiety, depression, and cognitive ability. Therefore, they propose that receiving head injury at an early age may prime the brain to be less susceptible to the effects of a later neurotrauma, a theory known as 'pre-conditioning' (Mychasiuk et al 2014). DeRoss, et al also showed that while one concussive impact resulted in diminished performance in $85 \%$ of animals, less deviation was seen with subsequent impacts. The number of impacts has an inverse relationship with animal performance in the water maze (DeRoss et al 2002). Again, the mechanism behind these findings is not entirely clear as those animals sustaining multiple injuries also received additional exposure to the water maze, allowing for enhanced training/learning of the maze (DeRoss et al 2002). Therefore, while repetitively injured animals did better than single-injured animals in the maze, this is likely a product of increased training rather than a protective response but this cannot be said with absolute certainty.

\section{Severity of impacts}

The effect of injury severity on likelihood of neurodegenerative disease development is not entirely clear, although some clinical reports indicate that more severe injury results in a greater predisposition for AD development (Plassman et al 2000, Uryu 
et al 2002). What is known from preclinical studies using an array of animal models is that there is a dose-dependent increase in neural injury markers and cognitive deficits with more severe injury (Budde et al 2013, DeFord et al 2002, Long et al 2009, Luo et al 2014a, Manley et al 2006, Turner et al 2013). Similarly, repetitive 'mild' injuries may produce a phenotype more consistent with a single more severe injury (DeFord et al 2002).

How 'mild' TBI contributes to the likelihood of developing CTE remains unclear. Emerging evidence from preclinical studies raises concern about lasting effects of subthreshold injuries when sustained in a rapid and repetitive fashion. These repetitive injuries contribute to vascular reactivity and subsequent axonal degeneration in vivo (Fujita et al 2012). Some studies suggest that severity of injury may dictate the rest period required to minimize cumulative cognitive deficits, although further studies are required to validate these findings (Meehan et al 2012).

Similarly, preclinical studies, even those in which only a mild force is imparted to a thinned cranium, indicate that a substantive inflammatory response is produced quickly after injury (Roth et al 2014). This response is associated with heightened vascular permeability, also seen clinically, as well as microglial response (Roth et al 2014). It is these mechanisms, both primary and secondary, that may contribute to neurodegenerative disease post-neurotrauma.

In vitro studies may be of further use in addressing the role of injury severity, particularly in subconcussive/subthreshold-type studies, as levels of injury and subsequent cellular responses can be monitored rapidly and performed over a greater 
number of iterations at a lower cost in comparison to in vivo studies. In fact, a number of injury paradigms and mechanisms have been investigated successfully in this manner including fluid pulse-induced shear stress, repetitive stretching, and other mechanical deformation procedures, both in vitro and ex vivo (LaPlaca \& Thibault 1998, Morrison et al 2006, Mukhin et al 1998, Sieg et al 1999, Slemmer \& Weber 2005, Slemmer et al 2004). The in vitro shearing studies found a significant amount of axonal beading and glial death (LaPlaca et al 2005).

\section{Age at time of impacts}

Age is the biggest risk factor for the development of neurodegenerative disease and has been associated with poor outcomes following TBI in a variety of clinical and preclinical reports (Anderson et al 2009, Hamm et al 1991, Hoane et al 2004, Maughan et al 2000, Mehan \& Strauss 2012, Timaru-Kast et al 2012, Turner et al 2012c, Turner et al 2014, Wali et al 2011). Similarly, neurotrauma has been associated with an increased risk of neurodegenerative disease development with regards to AD (Magnoni \& Brody 2010, Plassman et al 2000, Sivanandam \& Thakur 2012, Van Den Heuvel et al 2007), PD (Bower et al 2003), and CTE (Gavett et al 2011a, Goldstein et al 2012, McKee et al 2010, Montenigro et al 2014, Omalu 2014, Omalu et al 2011a, Omalu et al 2011b, Omalu et al 2010a, Omalu et al 2006, Omalu et al 2005, Omalu et al 2010b, Omalu et al 2010c, Stern et al 2013). One of the primary questions currently in the field is how the age at which the patient sustains the neurotrauma pertains to the development, or lack thereof, of CTE.

Similar findings have been observed in preclinical studies. Mychasiuk and colleagues showed that TBI during brain development leads to worse outcomes than TBI 
affecting the mature brain (Mychasiuk et al 2015). Preclinical work has also shown that young animals experience less edema than middle-aged animals following TBI (Kasturi \& Stein 2009). The increased edema is associated with an increase in lesion size in aged rodents experiencing TBI (Kumar et al 2013). TBI in aged rodents is also more likely to increase sensorimotor and cognitive decline (Hoane et al 2004). TBI in youth may ultimately be more detrimental for social development, whereas severe injury in the elderly results in rapid cognitive decline due to increased edema and therefore, lesion size. In regards to human TBI, it is unclear if the elderly would have a more progressive form of the disease similar to rodent studies or if the disease would develop in the normal manner. It is also important to consider that males $<35$ years old are the most likely to have repetitive TBIs, a group associated with heavy participation in sports and now the military (Theadom et al 2014). Future preclinical studies should therefore continue to investigate TBI secondary mechanisms in both young and aged animals with particular attention paid to addressing repetitive injury paradigms and the development of CTE-like features, both behaviorally and biochemically.

\section{Mechanism of impact}

CTE has been diagnosed in athletes sustaining direct impacts as a result of participation in warfare, football, wrestling, and soccer (Gavett et al 2011a, Goldstein et al 2012, McKee et al 2010, Montenigro et al 2014, Omalu 2014, Omalu et al 2011a, Omalu et al 2011b, Omalu et al 2010a, Omalu et al 2006, Omalu et al 2005, Omalu et al 2010b, Omalu et al 2010c, Stern et al 2013). The mechanism of injury is different between blast and athletic concussions, but how these mechanisms relate to injury progression remains to be elucidated. For blast, primary to quaternary injury must be 
considered (Kobeissy et al 2013). Other important questions include what is the influence of linear versus rotational impacts? What is the effect of direct impacts such as a football tackle versus indirect impacts such as primary blast exposure? Furthermore, how can comparisons most accurately be made across these various impacts? Are accelerometers and recording systems (such as the HITS system) the best method for understanding and comparing these impacts? What role does high-speed videography and subsequent kinematic analysis play? Each of these questions remains to be answered and may provide further insight into understanding the role that impact type plays in CTE development.

\section{Biochemical mechanisms}

Interestingly, glymphatic clearance has recently been shown to play a role in injury progression. Iliff and colleagues showed increased tauopathy accumulation in aquaporin knock-out mice following traumatic brain injury due to disrupted glymphatic clearance (Iliff et al 2014). It has yet to be determined how the primary injury mechanism causes the disruption to glymphatic channels. Cernak proposed an interesting theory about low-frequency stress waves transmitting kinetic energy through tissue (Cernak 2015). This mechanism may account for the dysfunction of the aquaporin channels. The energy transfer may also injure other cellular components such as axons or vessels. Chodobski and colleagues have shown that kinetic transfer of energy can account for blood brain barrier disruption (Chodobski et al 2011). Blood brain barrier disruption postinjury can trigger increased neuroinflammation. Agoston and colleagues showed that blast traumatic brain injury, in particular, causes persistent neuroinflammation, which

leads to behavioral deficits (Kovesdi et al 2012). The neuroinflammation can also 
contribute to post-traumatic epilepsy and tauopathy (Salazar \& Grafman 2015). Investigating how tauopathy spreads is a topic warranting further investigation (Koliatsos $\& \mathrm{Xu} 2015)$. Furthermore, Kobeissy and colleagues highlight in their recent review that underlying neuronal damage can cause lasting neuropsychiatric deficits such as postconcussion syndrome and post-traumatic stress disorder (Kobeissy et al 2013).

\section{Role of genetics}

Genetics and lifestyle choices may play a role in likelihood of sustaining a TBI and also the outcome following a TBI. Little to no evidence currently exists regarding lifestyle choices associated with TBI and only recently has genetic contribution to CTE been addressed. Specifically, it is well known in the human literature that the APOE4 allele is associated with worse outcome following TBI (Ponsford et al 2011). APOE4 and APO\&3 have also been implicated in the development of both CTE and AD (Saulle \& Greenwald 2012, Weber 2007). The mechanism by which APOe4 worsens outcome following TBI is poorly defined (Mannix et al 2013), but targeted replacement of the allele in mice has allowed focused research into cholesterol metabolism and may lead to insights in the field of traumatic brain injury and subsequent neurodegeneration (Laskowitz \& Dawson 2014). Notably, a study by Maroon and colleagues recently demonstrated that there was no significant difference between ApoE4 carriers in a population of patients afflicted with CTE when compared to the general population, suggesting that perhaps ApoE may not represent a significant risk factor for CTE development, even in individuals exposed to neurotrauma (Maroon et al 2015). 
Other genetic influences have also been observed but are currently limited to preclinical evidence. Rare genetic alterations such as mutation of the CACNAIA calcium subunit gene have also been shown to lead to poor TBI outcomes in human patients (Kors et al 2001). Recent pre-clinical studies have shed light on other potential genetic factors that may influence TBI outcomes. Decreases in micro RNA 23a and 27a increase apoptosis following TBI in rodents (Sabirzhanov et al 2014). Deficient caveolin expression can exacerbate neuroinflammation post-TBI (Niesman et al 2014). The knock-in mouse APP696swe has accelerated deposition of A $\beta$ following TBI (Yoshiyama et al 2005). Emerging evidence also suggests dysfunctional mitochondrial genes following TBI such as Fas, Apaf1, and Chp. Interestingly, these genes become more dysfunctional and mutated with time after mild TBI (Sharma et al 2012). TBI can also induce DNA fragmentation leading to an upregulation of p53, a critical regulator of cell cycle (Lu et al 2000). On the other hand, upregulation of insulin growth factor expression prior to TBI is associated with neuroprotection (Carlson et al 2014). Similarly, genetic regulation of aquaporin 4 channels can reduce edema formation following TBI in rodent models (Ke et al 2001). Surprisingly, disruption or the PARPI gene offers protection against TBI hypoxia (Hagberg et al 2004). Studying genetic risk factors for TBI is an area of growing importance and requires further investigation. Understanding factors that lead to increased or decreased TBI severity may also allow the development of novel pharmaceutics for the prevention of neurodegenerative disease.

An emerging area is the role of epigenetic modulation following TBI. Epigenetic markers are now being used preliminarily to predict recovery following injury (Lipsky \& Lin 2015). VandeVord and colleagues show enhanced methylation of DNA in the rat 
hippocampus following blast traumatic brain injury (Bailey et al 2015a). These epigenetic changes are mediated by HDACs and DNMTs (Bailey et al 2015b). Interestingly, the methylation changes are cumulative with repetitive injury (Haghighi et al 2015). HDAC has been shown to contribute to GSK3 $\beta$ activation, which is a known tau kinase. When HDAC is inhibited white matter damage is reduced (Wang et al 2015). Epigenetic regulation has been tied to the development of post-traumatic stress disorder clinically (Almli et al 2015). Targeting epigenetic regulation may therefore be a viable target in preventing tauopathy and behavioral deficits following traumatic brain injury.

\section{Influence of gender}

The influence of gender on outcome after TBI remains controversial, particularly in light of the few cases of CTE diagnosed in women. Some studies claim that females have better outcomes following neural injury (Bramlett \& Dietrich 2001) while others report no change (Bruce-Keller et al 2007, Kadyan et al 2004), or worse outcome (Farace \& Alves 2000a). Estrogen treatment has shown improved outcomes in rodent models of neural injury (Jia et al 2009, Li et al 2011, Simpkins et al 1997, Suzuki et al 2007), including TBI (Day et al 2013, Gatson et al 2012). Female rodents exhibit better outcomes after neural injury as evidenced by increased neurotrophin production (Gatson et al 2012), decreased neuroinflammation (Brown et al 2009), and better performance on motor tasks (Wagner et al 2004b). Clinical evidence shows that female patients exhibit lower oxidative damage after TBI (Wagner et al 2004a), which could be the result of a higher estrogen circulation following injury. Gender differences should be considered when conducting clinical trials for TBI therapy. 
Interestingly, Dixon and colleagues found that TBI in female humans reduces estradiol in the CSF (Garringer et al 2013). The reduction of estradiol may have unique long-term effects because it is not yet known if this is transient or permanent. The limited amount of data about women with TBI has restricted the comparisons of injury between genders (Bell \& Pepping 2001). The majority of traumatic brain injuries in women are subdural hematomas from falls. Elderly women with subdural hematomas tend to fair worse than the general TBI population as a whole (Farace \& Alves 2000b). Menopause may therefore dampen the neuroprotective physiologic properties mediated by estrogen post-injury. TBI in elderly women is also linked with earlier onset Alzheimer's disease (Nemetz et al 1999). Hormonal changes may also be a contributing factor to CTE, but this has yet to be verified. It is clear based on the limited pre-clinical and clinical data that further investigation into the gender differences surrounding TBI outcome is warranted.

\section{Effect of environment}

Another area of investigation required for elucidation of factors influencing the likelihood and/or severity of CTE development following neurotrauma includes the effect of environment. Areas of particular interest include social support, diet, use of supplements, and use of drugs or anabolic steroids.

Good social support has been shown to decrease the likelihood of developing a postconcussional disorder following acute head injury (McCauley et al 2001). Strong family support is linked to better outcomes (Khan et al 2003). Another important feature of environment is diet. While the effect of diet is well understood with regards to general health, there are limited studies relating diet and mental health, particularly in the context 
of traumatic brain injury. A preclinical study conducted by Mychasiuk and colleagues showed that high-fat diet in conjunction with TBI resulted in cumulative deficits on assessments of motor function, short-term working memory, and produced depressivelike effects compared to animals with normal diet and TBI (Mychasiuk et al 2014). Similarly, administration of dietary supplements such as Vitamin E and DHA have been shown to improve outcomes from TBI in numerous studies (Bailes \& Patel 2014, Conte et al 2004, Mills et al 2011a, Mills et al 2011b, Wu et al 2004). The suggestion has been made previously, based on evidence that some cases of diagnosed CTE occurred in former athletes with a history of anabolic steroid use, that anabolic steroid use may predispose these athletes to CTE development. While this question has not been completely resolved in terms of studying markers of CTE, preliminary studies found no difference in amyloid precursor protein (APP) expression post-TBI regardless of when anabolic steroids were used (Mills et al 2012).

\section{Preconditioning}

Any neural injury model has the potential to be complicated by the concept of preconditioning. Preconditioning at the most basic levels refers to neuroprotection for a given injury induced by a prior stimulus/injury. The concept of preconditioning has been well documented in a variety of neural injury models ranging from ischemic stroke to TBI (Weber 2007). Initial stimuli that serve a protective effect in a subsequent injury include but are not limited to brief periods of ischemia, chronic exposure to moderate heat or heat acclimation, and subthreshold or mild injury (Weber 2007). The proposed mechanism is that neuronal antioxidant machinery is upregulated with subthreshold injury thereby increasing the cells ability to respond to free radical production during 
subsequent injuries (Hu et al 2008) Whether preconditioning serves a protective phenomenon in the development of CTE is unclear, but the general clinical consensus is that any brain injury no matter how small may be detrimental long-term, minimizing any potential benefit of the preconditioning phenomenon (Bailes et al 2013, Talavage et al 2014). This is in contrast to preclinical literature demonstrating that sustaining repetitive mild injury prior to a single severe injury protects the animal from the most deleterious effects of the severe injury (Allen et al 2000). In other words, animals receiving repetitive mild injury prior to a severe injury do better than animals receiving a single severe injury (Allen et al 2000). Similar findings have been observed in vitro in which subthreshold stretch prevented more deleterious injury when a threshold stimulus was given (Slemmer \& Weber 2005). The concept of preconditioning versus additive injury is captured pictorially in figure 7.2.

\section{SUMMARY}

In conclusion, understanding $\mathrm{CTE}$ as a disease remains in its infancy and current studies remain largely speculative in nature without prospective clinical investigation. The required clinical studies to advance the field mandate extensive financial resources and time. Preclinical studies represent the most promising mechanism for studying many of the basic biologic questions about CTE, as discussed above. While these studies are continuing to evolve, numerous groups have reported exciting findings. Better modeling has allowed more extensive biochemical and behavioral characteristics to be defined. Now that our laboratory and others have established CTE models, options for translational investigation of CTE pathophysiology abound. In this work we discussed numerous avenues for addressing translational questions, namely the role of 1) inter- 
injury interval, 2) number of impacts, 3) impact severity, 4) age at time of impacts, 5) mechanism of impact, 6) genetics, 7) gender, and 8) effect of environment on the development of CTE. We also highlighted some of the challenges of CTE modeling and specific requirements for successful models. By improving our understanding about CTE mechanisms, we believe that significant strides can be made not only in understanding CTE but also potentially developing prevention and therapeutic-related approaches. A companion manuscript describes our collective experience in modeling CTE, both neuropathologically and behaviorally.

\section{ACKNOWLEDGEMENTS}

A Research Funding and Development Grant from West Virginia University supported the project. An American Medical Association Foundation Seed-Grant, a Neurosurgery Research and Education Foundation Medical Student Summer Research Fellowship, and an American Foundation of Pharmaceutical Education Pre-doctoral Fellowship supported Brandon Lucke-Wold. An American Foundation of Pharmaceutical Education Predoctoral Fellowship also supported Aric Logsdon. 


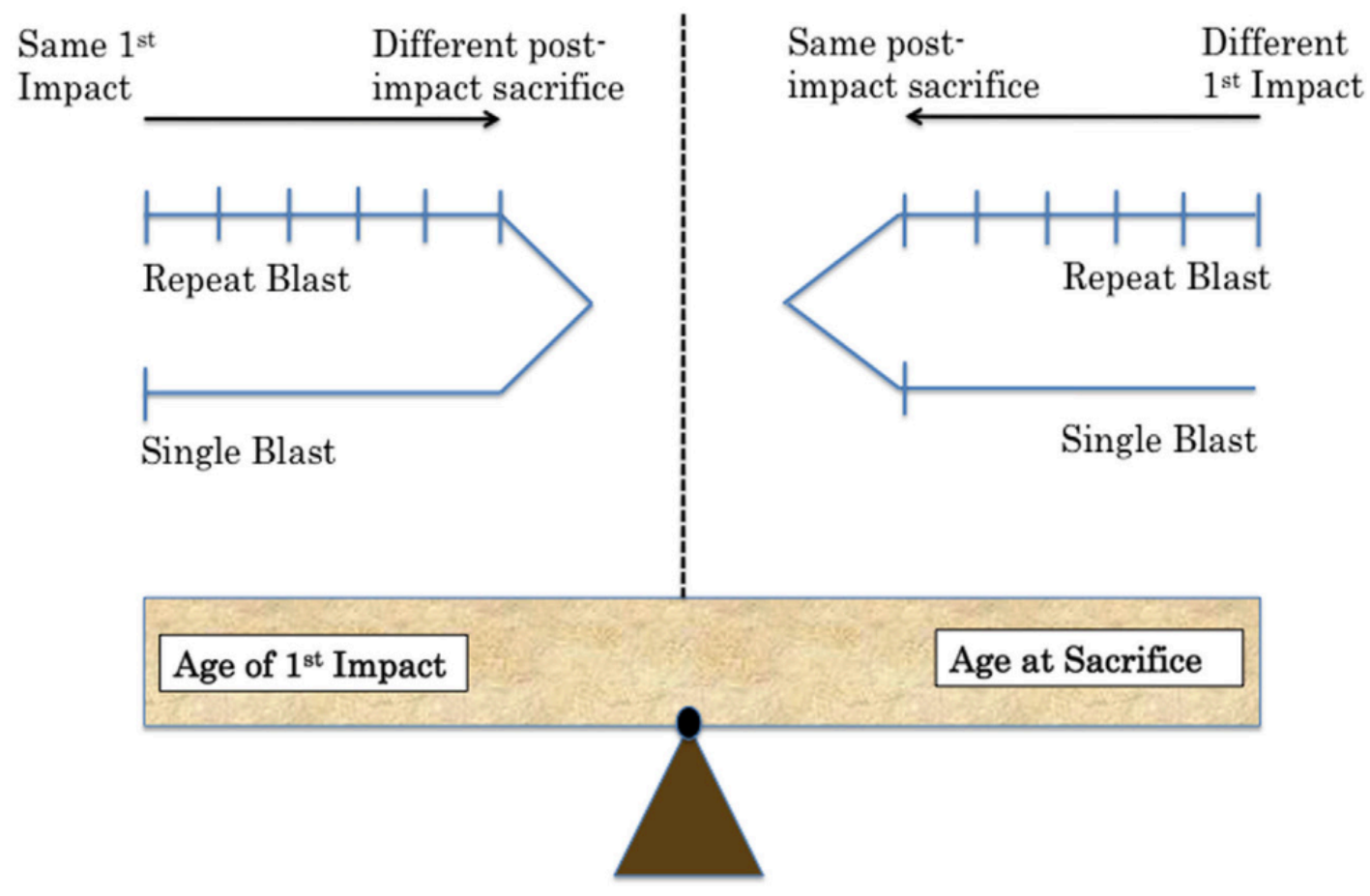

Figure 7.1: Methodological challenges associated with repeat injury in comparison to single injury paradigms include balancing equal age at time of exposure versus age at sacrifice. Two possibilities are shown depending on the variable the experimenter wants to control in future work. 


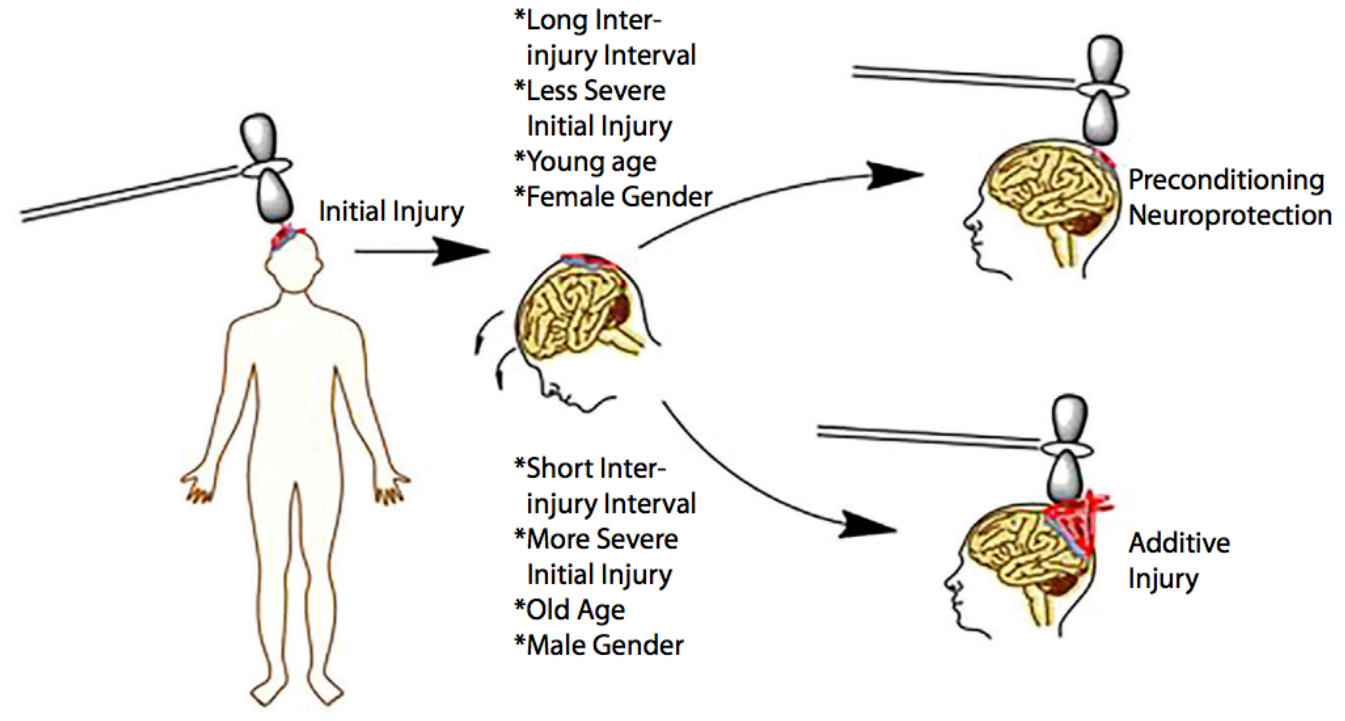

Figure 7.2: Schematic representation of factors influencing injury outcomes, particularly in repetitive injury paradigms. Initial evidence indicates that longer inter-injury intervals, less severe initial injury, a younger age, and female gender may serve as protective effects in repeat paradigms. In contrast, shorter intervals, increased severity, older age, and male gender may be associated with worse outcomes. 
TABLE 1 | Chronic traumatic encephalopathy TBI models.

\begin{tabular}{|c|c|c|c|c|c|c|}
\hline Study & Sex/species/age & Model & Injuries & Interval & Anesthesia & Outcome measures \\
\hline $\begin{array}{l}\text { Liu } \\
\text { et al. (27) }\end{array}$ & $\begin{array}{l}\text { Male } \\
\text { S. Dawley rat } \\
2-3 \text { months }\end{array}$ & $\begin{array}{l}\text { Metal CCl } \\
\text { Open head }\end{array}$ & Single & Single & Isoflurane & Tauopathy; cell death; apoptosis \\
\hline $\begin{array}{l}\text { Goldstein } \\
\text { et al. (17) }\end{array}$ & $\begin{array}{l}\text { Male } \\
\text { C57BL6 mice } \\
2-3 \text { months }\end{array}$ & $\begin{array}{l}\text { Blast } \\
\text { Closed head }\end{array}$ & Single & Single & $\begin{array}{l}\text { Ketamine/ } \\
\text { xylazine }\end{array}$ & $\begin{array}{l}\text { Electrophysiology; tauopathy; axonal damage; } \\
\text { motor; cognition; structural integrity; advanced } \\
\text { imaging; human studies }\end{array}$ \\
\hline $\begin{array}{l}\text { Ojo et al. } \\
(18)\end{array}$ & $\begin{array}{l}\text { Male/female } \\
\text { C57BL6 mice, hTau Tg mice } \\
18 \text { months }\end{array}$ & $\begin{array}{l}\text { Metal CCl } \\
\text { Closed head }\end{array}$ & $\begin{array}{l}\text { Single } \\
\text { Repeat (5) }\end{array}$ & Single (48 h) & Isoflurane & $\begin{array}{l}\text { Tauopathy; gliosis and degeneration; structural } \\
\text { integrity; cell death }\end{array}$ \\
\hline $\begin{array}{l}\text { Mouzon } \\
\text { et al. (25) }\end{array}$ & $\begin{array}{l}\text { Male } \\
\text { C57BL6 mice } \\
\text { 9-15 months }\end{array}$ & $\begin{array}{l}\text { Metal CCl } \\
\text { Closed head }\end{array}$ & $\begin{array}{l}\text { Single } \\
\text { Repeat (5) }\end{array}$ & Single (48 h) & Isoflurane & $\begin{array}{l}\text { Motor; cognition; anxiety; inflammation; tauopathy; } \\
\text { axonal damage }\end{array}$ \\
\hline $\begin{array}{l}\text { Huber } \\
\text { et al. (26) }\end{array}$ & $\begin{array}{l}\text { Male } \\
\text { C57BL6 mice } \\
\text { 2-3 months }\end{array}$ & $\begin{array}{l}\text { Blast } \\
\text { Closed head }\end{array}$ & Single & Single & Isoflurane & Motor; oxidative stress; tauopathy \\
\hline $\begin{array}{l}\text { Luo et al. } \\
\text { (22) }\end{array}$ & $\begin{array}{l}\text { Male } \\
\text { C57BL6 mice, } \\
\text { GFAP Luc mice } \\
2-3 \text { months }\end{array}$ & $\begin{array}{l}\text { Rubber } \mathrm{CCl} \\
\text { Closed head }\end{array}$ & $\begin{array}{l}\text { Single } \\
\text { Repeat } \\
(2,3,5)\end{array}$ & Single (24 h) & Isoflurane & $\begin{array}{l}\text { Bioluminescence; motor; anxiety; cognition; fear } \\
\text { conditioning; gliosis and degeneration; apoptosis }\end{array}$ \\
\hline $\begin{array}{l}\text { Glushakova } \\
\text { et al. (39) }\end{array}$ & $\begin{array}{l}\text { Male } \\
\text { S. Dawley rats } \\
2-3 \text { months }\end{array}$ & $\begin{array}{l}\text { Metal CCI } \\
\text { Open head }\end{array}$ & Single & Single & Isoflurane & $\begin{array}{l}\text { Vascular and axonal damage; gliosis and } \\
\text { degeneration }\end{array}$ \\
\hline $\begin{array}{l}\text { Zhang } \\
\text { et al. (32) }\end{array}$ & $\begin{array}{l}\text { Male } \\
\text { C57BL6 mice } \\
\text { 2-3 months }\end{array}$ & $\begin{array}{l}\text { Metal CCl } \\
\text { Closed head }\end{array}$ & $\begin{array}{l}\text { Single } \\
\text { Repeat (3) }\end{array}$ & Single (24 h) & Avertin & $\begin{array}{l}\text { Electrophysiology; neuroscore; inflammation; } \\
\text { tauopathy; gliosis and degeneration; cognition }\end{array}$ \\
\hline $\begin{array}{l}\text { Kondo } \\
\text { et al. (29) }\end{array}$ & $\begin{array}{l}\text { Male } \\
\text { C57BL6 mice } \\
2-3 \text { months }\end{array}$ & $\begin{array}{l}\text { Single } \\
\text { blast and } \\
\text { weightdrop } \\
\text { Closed head }\end{array}$ & $\begin{array}{l}\text { Single } \\
\text { Repeat (7) }\end{array}$ & $\begin{array}{l}\text { Single severe } \\
\text { Seven mild } \\
\text { over } 9 \text { days }\end{array}$ & Isoflurane & $\begin{array}{l}\text { Electrophysiology; motor; cognition; anxiety; } \\
\text { structural integrity; axonal damage; tauopathy; cell } \\
\text { death; mitochondrial function; human studies }\end{array}$ \\
\hline $\begin{array}{l}\text { Lucke-Wold } \\
\text { et al. }(24 \text {, } \\
\text { 30) }\end{array}$ & $\begin{array}{l}\text { Male } \\
\text { S. Dawley rats } \\
2-3 \text { months }\end{array}$ & $\begin{array}{l}\text { Blast } \\
\text { Closed head }\end{array}$ & $\begin{array}{l}\text { Single } \\
\text { Repeat (6) }\end{array}$ & $\begin{array}{l}\text { Single } \\
\text { Six mild over } \\
10 \text { days }\end{array}$ & Isoflurane & $\begin{array}{l}\text { Cognition; endoplasmic reticulum stress; tauopathy; } \\
\text { human studies }\end{array}$ \\
\hline
\end{tabular}

Table 7.1: A collection of studies that propose to model CTE. The majority of the studies represent both neuropathologic and behavioral deficits. Tauopathy, behavioral changes, and worse cognitive performance are hallmarks. 


\begin{tabular}{|c|c|c|c|c|c|c|}
\hline Study & Sex/species/age & Model & Injuries & Interval & Anesthesia & Outcome measures \\
\hline $\begin{array}{l}\text { Long } \\
\text { et al. (74) }\end{array}$ & $\begin{array}{l}\text { Male } \\
\text { S. Dawley rats } \\
2-3 \text { months }\end{array}$ & $\begin{array}{l}\text { 5.3-m metal } \\
\text { tube }\end{array}$ & Single & Single & Isoflurane & $\begin{array}{l}\text { Cardiovascular; motor; cognition; structural integrity; } \\
\text { vascular damage; degeneration }\end{array}$ \\
\hline $\begin{array}{l}\text { Budde } \\
\text { et al. (75) }\end{array}$ & $\begin{array}{l}\text { Unknown } \\
\text { S. Dawley rats } \\
\text { Unknown }\end{array}$ & $\begin{array}{l}\text { 3.3-m metal } \\
\text { tube }\end{array}$ & $\begin{array}{l}\text { Single mild } \\
\text { and severe }\end{array}$ & Single & Isoflurane & $\begin{array}{l}\text { Advanced imaging; anxiety; cognition; gliosis and } \\
\text { degeneration; apoptosis }\end{array}$ \\
\hline $\begin{array}{l}\text { Genovese } \\
\text { et al. }(50)\end{array}$ & $\begin{array}{l}\text { Male } \\
\text { S. Dawley rats } \\
2-3 \text { months }\end{array}$ & $\begin{array}{l}\text { 5.3-m metal } \\
\text { tube }\end{array}$ & Repeat (3) & $24 \mathrm{~h}$ & Isoflurane & Fear conditioning \\
\hline $\begin{array}{l}\text { Wang et al. } \\
\text { (52) }\end{array}$ & $\begin{array}{l}\text { Male } \\
\text { C57BL6 mice } \\
\text { 2-3 months }\end{array}$ & $\begin{array}{l}5.3-\mathrm{m} \text { metal } \\
\text { tube }\end{array}$ & Repeat (3) & 1 or $30 \mathrm{~min}$ & Isoflurane & $\begin{array}{l}\text { Mitochondrial function; DNA fragmentation; righting } \\
\text { reflex; apoptosis }\end{array}$ \\
\hline $\begin{array}{l}\text { Lucke-Wold } \\
\text { et al. }(6,42)\end{array}$ & $\begin{array}{l}\text { Male } \\
\text { S. Dawley rats } \\
2-3 \text { months }\end{array}$ & $\begin{array}{l}\text { 0.3-m metal } \\
\text { tube }\end{array}$ & Single & Single & Isoflurane & $\begin{array}{l}\text { Vascular damage; structural integrity; gliosis and } \\
\text { degeneration }\end{array}$ \\
\hline $\begin{array}{l}\text { Logsdon et al. } \\
\text { (41) }\end{array}$ & $\begin{array}{l}\text { Male } \\
\text { S. Dawley rats } \\
2-3 \text { months }\end{array}$ & $\begin{array}{l}\text { 0.3-m metal } \\
\text { tube }\end{array}$ & Single & Single & Isoflurane & $\begin{array}{l}\text { Vascular damage; endoplasmic reticulum stress; cell } \\
\text { death; apoptosis; anxiety }\end{array}$ \\
\hline
\end{tabular}

Table 7.2: A collection of studies utilizing blast-induced neurotrauma models are summarized here. These studies employed both single and repeat-injury paradigms with experimental endpoints investigating both neurobehavioral changes and injury pathophysiology. Notably, the referenced studies, consistent with most blast literature, have been conducted within the past 5 years, indicating that blast pathophysiology is probably the least well understood of any mechanism of neurotrauma. 


\begin{tabular}{|c|c|c|c|c|c|c|}
\hline Study & Sex/species/age & Model & Injuries & Interval & Anesthesia & Outcome measures \\
\hline $\begin{array}{l}\text { DeFord } \\
\text { et al. (72) }\end{array}$ & $\begin{array}{l}\text { Male } \\
\text { C57BL6 mice } \\
\text { 2-3 months }\end{array}$ & $\begin{array}{l}\text { Weight-drop } \\
\text { Closed head }\end{array}$ & $\begin{array}{l}\text { Single } \\
\text { Repeat (4) }\end{array}$ & Single (24 h) & $\begin{array}{l}\text { Isoflurane } \\
\mathrm{N}_{2} \mathrm{O} \text { and } \mathrm{O}_{2}(70: 30)\end{array}$ & $\begin{array}{l}\text { Neuroscore; cell death; vascular } \\
\text { damage; cardiovascular; cognition }\end{array}$ \\
\hline $\begin{array}{l}\text { Creeley } \\
\text { et al. (48) }\end{array}$ & $\begin{array}{l}\text { Male } \\
\text { C57BL6 mice } \\
\text { 2-3 months }\end{array}$ & $\begin{array}{l}\text { Weight-drop } \\
\text { Closed head }\end{array}$ & Repeat (3) & $24 \mathrm{~h}$ & Isoflurane & $\begin{array}{l}\text { Motor; cognition; righting reflex; } \\
\text { cell death }\end{array}$ \\
\hline $\begin{array}{l}\text { Fujita } \\
\text { et al. (53) }\end{array}$ & $\begin{array}{l}\text { Male } \\
\text { S. Dawley rats } \\
3-6 \text { months }\end{array}$ & $\begin{array}{l}\text { Weight-drop } \\
\text { Skull-exposed }\end{array}$ & $\begin{array}{l}\text { Single } \\
\text { Repeat mild }(2,3) \\
\text { Repeat medium (2) } \\
\text { Repeat severe (2) }\end{array}$ & $\begin{array}{l}\text { Single } \\
\text { Two mild over } 3 \mathrm{~h} \\
\text { Three mild over } 2 \mathrm{~h} \\
\text { Two medium over } 3 \mathrm{~h} \\
\text { Two severe over } 3 \mathrm{~h} \\
\text { Two severe over } 5 \mathrm{~h} \\
\text { Two severe over } 10 \mathrm{~h}\end{array}$ & Pentobarbital & $\begin{array}{l}\text { Vascular reactivity to } \mathrm{ACh} \text {; axonal } \\
\text { damage }\end{array}$ \\
\hline $\begin{array}{l}\text { Meehan } \\
\text { et al. (54) }\end{array}$ & $\begin{array}{l}\text { Male } \\
\text { C57BL6 mice } \\
\text { 2-3 months }\end{array}$ & $\begin{array}{l}\text { Weight-drop } \\
\text { Closed head }\end{array}$ & $\begin{array}{l}\text { Single } \\
\text { Repeat daily }(3,10) \\
\text { Repeat variable }(5)\end{array}$ & $\begin{array}{l}\text { Single } \\
\text { Daily }(3,5, \text { or } 10) \\
\text { Weekly (5) } \\
\text { Monthly (5) }\end{array}$ & $\begin{array}{l}\text { Isoflurane } \\
\mathrm{N}_{2} \mathrm{O} \text { and } \mathrm{O}_{2}(70: 30)\end{array}$ & $\begin{array}{l}\text { Edema; axonal and vascular } \\
\text { damage; cell death; cognition }\end{array}$ \\
\hline $\begin{array}{l}\text { Mannix } \\
\text { et al. (54) }\end{array}$ & $\begin{array}{l}\text { Male } \\
\text { C57BL6 mice } \\
\text { 2-3 months }\end{array}$ & $\begin{array}{l}\text { Weight-drop } \\
\text { Closed head }\end{array}$ & $\begin{array}{l}\text { Single } \\
\text { Repeat daily }(5,7) \\
\text { Repeat variable }(5)\end{array}$ & $\begin{array}{l}\text { Single } \\
\text { Daily (5 or } 7 \text { ) } \\
\text { Weekly (5) } \\
\text { Biweekly (5) } \\
\text { Monthly (5) }\end{array}$ & $\begin{array}{l}\text { Isoflurane } \\
\mathrm{N}_{2} \mathrm{O} \text { and } \mathrm{O}_{2}(70: 30)\end{array}$ & $\begin{array}{l}\text { Cognition; tauopathy; advanced } \\
\text { imaging }\end{array}$ \\
\hline $\begin{array}{l}\text { Weil } \\
\text { et al. (57) }\end{array}$ & $\begin{array}{l}\text { Male } \\
\text { Swiss web. mice } \\
2-3 \text { months }\end{array}$ & $\begin{array}{l}\text { Weight-drop } \\
\text { Skull-exposed }\end{array}$ & $\begin{array}{l}\text { Single } \\
\text { Repeat (2) }\end{array}$ & Single 3 or 20 days & Isoflurane & $\begin{array}{l}\text { Glucose metabolism; } \\
\text { inflammation; gliosis and } \\
\text { degeneration; cell death; cognition }\end{array}$ \\
\hline
\end{tabular}

Table 7.3: Selected studies using a conventional weight-drop model similar to the Marmarou or impact-acceleration injury models utilized both single and repeat-injury paradigms. 


\begin{tabular}{|c|c|c|c|c|c|c|}
\hline Study & Sex/species/age & Model & Injuries & Interval & Anesthesia & Outcome measures \\
\hline $\begin{array}{l}\text { Mouzon } \\
\text { et al. (65) }\end{array}$ & $\begin{array}{l}\text { Male } \\
\text { C57BL6 mice } \\
\text { 2-3 months }\end{array}$ & $\begin{array}{l}\text { Metal CCl } \\
\text { Closed head }\end{array}$ & $\begin{array}{l}\text { Single } \\
\text { Repeat (5) }\end{array}$ & Single (48 h) & Isoflurane & $\begin{array}{l}\text { Motor; cognition; gliosis and degeneration; righting } \\
\text { reflex; axonal damage }\end{array}$ \\
\hline $\begin{array}{l}\text { Yoshiyama } \\
\text { et al. (35) }\end{array}$ & $\begin{array}{l}\text { Male/Female } \\
\text { B6D2/F1 mice, } \\
\text { Tau Tg mice } \\
12 \text { months }\end{array}$ & $\begin{array}{l}\text { Silicone CCl } \\
\text { Skull-exposed }\end{array}$ & Repeat (16) & $\begin{array}{l}\text { Four per day } \\
\text { Every } 20 \text { min } \\
\text { Once a week for } \\
4 \text { weeks }\end{array}$ & Isoflurane & $\begin{array}{l}\text { Neuroscore; cognition; gliosis and degeneration; } \\
\text { tauopathy }\end{array}$ \\
\hline $\begin{array}{l}\text { Laurer } \\
\text { et al. (64) }\end{array}$ & $\begin{array}{l}\text { Male } \\
\text { C57BL6 mice } \\
2-3 \text { months }\end{array}$ & $\begin{array}{l}\text { Rubber CCI } \\
\text { Skull-exposed }\end{array}$ & $\begin{array}{l}\text { Single } \\
\text { Repeat (2) }\end{array}$ & Single (24 h) & Pentobarbital & $\begin{array}{l}\text { Neuroscore; motor; cardiovascular; cognition; } \\
\text { axonal and vascular damage; cell death; tauopathy }\end{array}$ \\
\hline $\begin{array}{l}\text { Bolton and } \\
\text { Saatman (56) }\end{array}$ & $\begin{array}{l}\text { Male } \\
\text { C57BL6 mice } \\
\text { 2-3 months }\end{array}$ & $\begin{array}{l}\text { Silicone CCl } \\
\text { Skull-exposed }\end{array}$ & $\begin{array}{l}\text { Single } \\
\text { Repeat (5) }\end{array}$ & Single (24 or 48 h) & Isoflurane & $\begin{array}{l}\text { Cardiovascular; righting reflex; axonal damage; } \\
\text { gliosis and degeneration; tauopathy }\end{array}$ \\
\hline $\begin{array}{l}\text { Shitaka } \\
\text { et al. (49) }\end{array}$ & $\begin{array}{l}\text { Male } \\
\text { C57BL6 mice } \\
\text { 2-3 months }\end{array}$ & $\begin{array}{l}\text { Rubber CCI } \\
\text { Skull-exposed }\end{array}$ & Repeat (2) & $24 \mathrm{~h}$ & Isoflurane & $\begin{array}{l}\text { Cognition; structural integrity; gliosis and } \\
\text { degeneration; axonal damage; electron microscopy }\end{array}$ \\
\hline $\begin{array}{l}\text { Klemenhagen } \\
\text { et al. (51) }\end{array}$ & $\begin{array}{l}\text { Male } \\
\text { C57BL6 mice } \\
2-3 \text { months }\end{array}$ & $\begin{array}{l}\text { Rubber CCl } \\
\text { Skull-exposed }\end{array}$ & Repeat (2) & $24 \mathrm{~h}$ & Isoflurane & $\begin{array}{l}\text { Fear conditioning; cognition; social recognition; } \\
\text { depression; anhedonia; gliosis; vascular damage }\end{array}$ \\
\hline $\begin{array}{l}\text { Uryu } \\
\text { et al. (33) }\end{array}$ & $\begin{array}{l}\text { Male/Female } \\
\text { B6D2/F1 mice, } \\
\text { APP Tg mice } \\
\text { 9-12 months }\end{array}$ & $\begin{array}{l}\text { Rubber CCI } \\
\text { Skull-exposed }\end{array}$ & $\begin{array}{l}\text { Single } \\
\text { Repeat (2) }\end{array}$ & Single (24 h) & Pentobarbital & $\begin{array}{l}\text { Neuroscore; cognition; motor; vascular damage; } \\
\text { gliosis and degeneration; tauopathy; oxidative } \\
\text { stress }\end{array}$ \\
\hline $\begin{array}{l}\text { Longhi } \\
\text { et al. (47) }\end{array}$ & $\begin{array}{l}\text { Male } \\
\text { C57BL6 mice } \\
2-3 \text { months }\end{array}$ & $\begin{array}{l}\text { Silicone CCI } \\
\text { Skull-exposed }\end{array}$ & $\begin{array}{l}\text { Single } \\
\text { Repeat (2) }\end{array}$ & $\begin{array}{l}\text { Single }(3,5 \text {, or } \\
7 \text { days })\end{array}$ & Isoflurane & $\begin{array}{l}\text { Cognition; motor; righting reflex; gliosis and } \\
\text { degeneration; axonal and cytoskeletal damage; cell } \\
\text { death; edema }\end{array}$ \\
\hline $\begin{array}{l}\text { Conte } \\
\text { et al. (34) }\end{array}$ & $\begin{array}{l}\text { Female } \\
\text { B6D2/F1 mice, APP } \\
\text { Tg mice } \\
9-12 \text { months }\end{array}$ & $\begin{array}{l}\text { Rubber CCI } \\
\text { Skull-exposed }\end{array}$ & Repeat (2) & $24 \mathrm{~h}$ & Isoflurane & $\begin{array}{l}\text { Cognition; tauopathy; structural integrity; oxidative } \\
\text { stress }\end{array}$ \\
\hline
\end{tabular}

Table 7.4: Modification of the more traditional controlled cortical impact device has resulted in the ability to develop highly reproducible neurotrauma in preclinical models. Consequently, all of the selected studies demonstrate the implementation of repeat-injury paradigms, often in the investigation of CTE-like neurodegeneration and neurobehavioral changes. 
TABLE 5 | TBI models using craniotomy.

\begin{tabular}{|c|c|c|c|c|c|c|}
\hline Study & Sex/species/age & Model & Injuries & Interval & Anesthesia & Outcome measures \\
\hline $\begin{array}{l}\text { Olsson } \\
\text { et al. (68) }\end{array}$ & $\begin{array}{l}\text { Rabbits } \\
\text { Unknown }\end{array}$ & Fluid percussion & $\begin{array}{l}\text { Single } \\
\text { Repeat (10) }\end{array}$ & Single (5 min) & Pentobarbital & $\begin{array}{l}\text { Righting reflex; cardiovascular; } \\
\text { vascular damage }\end{array}$ \\
\hline $\begin{array}{l}\text { Smith } \\
\text { et al. (40) }\end{array}$ & $\begin{array}{l}\text { Male } \\
\text { C57BL6 mice } \\
2-3 \text { months }\end{array}$ & $\begin{array}{l}\text { Metal CCl } \\
\text { Open head }\end{array}$ & Single & Single & Pentobarbital & $\begin{array}{l}\text { Cognition; structural integrity; cell } \\
\text { death; gliosis and degeneration; } \\
\text { vascular damage }\end{array}$ \\
\hline $\begin{array}{l}\text { Kanayama } \\
\text { et al. (31) }\end{array}$ & $\begin{array}{l}\text { Male } \\
\text { Wister rats } \\
2-3 \text { months }\end{array}$ & Fluid percussion & $\begin{array}{l}\text { Single } \\
\text { Repeat (7) }\end{array}$ & Single $(24 \mathrm{~h})$ & Pentobarbital & $\begin{array}{l}\text { Motor; social recognition; cytoskeletal } \\
\text { damage; tauopathy }\end{array}$ \\
\hline $\begin{array}{l}\text { Allen } \\
\text { et al. (158) }\end{array}$ & $\begin{array}{l}\text { Male } \\
\text { S. Dawley rats } \\
2-3 \text { months }\end{array}$ & $\begin{array}{l}\text { Weight-drop } \\
\text { Plexiglas piston }\end{array}$ & $\begin{array}{l}\text { Single severe } \\
\text { Repeat mild (3) }\end{array}$ & $\begin{array}{l}\text { Three mild over } \\
14 \text { days } \pm \text { severe } \\
3 \text { days }\end{array}$ & $\begin{array}{l}\text { Pentobarbital } \\
\text { or ketamine/ } \\
\text { rhompamine }\end{array}$ & $\begin{array}{l}\text { Motor; gliosis and degeneration; } \\
\text { structural integrity }\end{array}$ \\
\hline $\begin{array}{l}\text { DeRoss } \\
\text { et al. (70) }\end{array}$ & $\begin{array}{l}\text { Male } \\
\text { Long-Evans rats } \\
2-3 \text { months }\end{array}$ & Fluid percussion & $\begin{array}{l}\text { Single } \\
\text { Repeat }(2,3)\end{array}$ & Single (N/A) & Isoflurane & Cognition; motor \\
\hline $\begin{array}{l}\text { Manley } \\
\text { et al. (73) }\end{array}$ & $\begin{array}{l}\text { Male } \\
\text { Yorkshire pigs } \\
\text { Adult }\end{array}$ & $\begin{array}{l}\text { Metal CCl } \\
\text { Open head }\end{array}$ & Single & Single & $\begin{array}{l}\text { Pancuronium } \\
\text { Isoflurane }\end{array}$ & $\begin{array}{l}\text { Cardiovascular; structural integrity; } \\
\text { edema; vascular damage; cell death; } \\
\text { gliosis and degeneration }\end{array}$ \\
\hline $\begin{array}{l}\text { Donovan } \\
\text { et al. (61) }\end{array}$ & $\begin{array}{l}\text { Male } \\
\text { S. Dawley rats } \\
2-3 \text { months }\end{array}$ & $\begin{array}{l}\text { Metal CCl } \\
\text { Open head }\end{array}$ & $\begin{array}{l}\text { Single } \\
\text { Repeat (2) }\end{array}$ & $\begin{array}{l}\text { Single } 7 \text { days } \\
\text { each side }\end{array}$ & Isoflurane & $\begin{array}{l}\text { Advanced imaging; structural } \\
\text { integrity; axonal damage }\end{array}$ \\
\hline $\begin{array}{l}\text { Hawkins } \\
\text { et al. (61) }\end{array}$ & $\begin{array}{l}\text { Male } \\
\text { S. Dawley Rats } \\
6-8 \text { months }\end{array}$ & Fluid percussion & Single & Single & Isoflurane & Extensive tauopathy assessment \\
\hline $\begin{array}{l}\text { Rubenstein } \\
\text { et al. (38) }\end{array}$ & $\begin{array}{l}\text { Male } \\
\text { S. Dawley rats and } \\
\text { C57BL6 mice } \\
2-3 \text { months }\end{array}$ & $\begin{array}{l}\text { Metal CCl } \\
\text { Open head }\end{array}$ & Single & Single & Isoflurane & $\begin{array}{l}\text { Extensive tauopathy assessment; } \\
\text { human studies }\end{array}$ \\
\hline $\begin{array}{l}\text { Begum } \\
\text { et al. (37) }\end{array}$ & $\begin{array}{l}\text { Male } \\
\text { S. Dawley rats } \\
2-3 \text { months }\end{array}$ & $\begin{array}{l}\text { Metal CCl } \\
\text { Open head }\end{array}$ & Single & Single & Isoflurane & $\begin{array}{l}\text { Motor; endoplasmic reticulum stress; } \\
\text { tauopathy; axonal damage }\end{array}$ \\
\hline $\begin{array}{l}\text { Aungst } \\
\text { et al. (67) }\end{array}$ & $\begin{array}{l}\text { Male } \\
\text { S. Dawley rats } \\
2-3 \text { months }\end{array}$ & Fluid percussion & $\begin{array}{l}\text { Single } \\
\text { Repeat (3) }\end{array}$ & Single (48 h) & Isoflurane & $\begin{array}{l}\text { Electrophysiology; neuroscore; } \\
\text { cognition; social recognition; gliosis } \\
\text { and degeneration; cell death }\end{array}$ \\
\hline
\end{tabular}

Table 7.5: Selected studies of interest requiring craniotomy for administration of fluidpercussion injury, controlled cortical impact, or open-head weight-drop. 


\begin{tabular}{|c|c|c|c|c|c|c|}
\hline Study & Sex/species/age & Model & Injuries & Interval & Anesthesia & Outcome measures \\
\hline $\begin{array}{l}\text { Raghupathi } \\
\text { et al. (69) }\end{array}$ & $\begin{array}{l}\text { Male } \\
\text { Farm pigs } \\
3-5 \text { days }\end{array}$ & $\begin{array}{l}\text { Non-impact } \\
\text { head rotation }\end{array}$ & $\begin{array}{l}\text { Single } \\
\text { Repeat }\end{array}$ & Single (15 min) & Isoflurane & $\begin{array}{l}\text { Cardiovascular; axonal and vascular damage; } \\
\text { structural integrity; cell death }\end{array}$ \\
\hline Friess et al. (58) & $\begin{array}{l}\text { Male } \\
\text { Farm pigs } \\
3-5 \text { days }\end{array}$ & $\begin{array}{l}\text { Non-impact } \\
\text { head rotation }\end{array}$ & $\begin{array}{l}\text { Single } \\
\text { Repeat (2) }\end{array}$ & $\begin{array}{l}\text { Single }(24 \mathrm{~h} \text { or } \\
7 \text { days }\end{array}$ & Isoflurane & Motor; cognition; axonal damage \\
\hline Roth et al. (77) & $\begin{array}{l}\text { Male } \\
\text { S. Dawley rats and } \\
\text { C57BL6 mice } \\
2-3 \text { months }\end{array}$ & $\begin{array}{l}\text { Skull thinning } \\
\text { compression } \\
\text { Skull-exposed }\end{array}$ & Single & Single & $\begin{array}{l}\text { Ketamine/xylazine/ } \\
\text { acepromazine }\end{array}$ & $\begin{array}{l}\text { Inflammation; gliosis and degeneration; oxidative } \\
\text { stress; vascular damage; structural integrity; } \\
\text { advanced imaging; human studies }\end{array}$ \\
\hline $\begin{array}{l}\text { Petraglia et al. } \\
(20,23)\end{array}$ & $\begin{array}{l}\text { Male } \\
\text { C57BL6 mice } \\
2-3 \text { months }\end{array}$ & $\begin{array}{l}\text { Rubber CCl } \\
\text { Helmet }\end{array}$ & $\begin{array}{l}\text { Single } \\
\text { Repeat (42) }\end{array}$ & $\begin{array}{l}\text { Six per day every } \\
2 \mathrm{~h} \text { over } 7 \text { days }\end{array}$ & No Anesthesia & $\begin{array}{l}\text { Neuroscore; motor; cognition; anxiety; depression; } \\
\text { sleep }\end{array}$ \\
\hline
\end{tabular}

Table 7.6: Less conventional models of neurotrauma due to species utilized, injury mechanism, and/or equipment adaptations. Of particular interest is the study by Petraglia and colleagues that administered single or repetitive impacts in unanesthetized animals. 
TABLE 7 | In vitro and ex vivo TBI models.

\begin{tabular}{|c|c|c|c|c|c|c|}
\hline Study & Model & Cell Line & Injuries & Interval & Severity & Outcome measures \\
\hline $\begin{array}{l}\text { Zander } \\
\text { et al. (43) }\end{array}$ & Primary blast & PC12 neurons & $\begin{array}{l}\text { Single } \\
\text { Repeat (3) }\end{array}$ & $\begin{array}{l}\text { Single } \\
\text { Three over } \\
20 \text { min }\end{array}$ & $\begin{array}{l}\text { Mild to } \\
\text { moderate }\end{array}$ & $\begin{array}{l}\text { Membrane permeability; cell viability; } \\
\text { axonal damage }\end{array}$ \\
\hline $\begin{array}{l}\text { LaPlaca and } \\
\text { Thibault (78) }\end{array}$ & Shear stress & Neuronal culture & Single & Single & Mild & $\begin{array}{l}\text { Membrane permeability; calcium influx; } \\
\text { cell death }\end{array}$ \\
\hline Morrison et al. (81) & Membrane strain & $\begin{array}{l}\text { Organo-typic } \\
\text { hippocampal slices }\end{array}$ & Single & Single & $\begin{array}{l}\text { Mild to } \\
\text { Severe }\end{array}$ & $\begin{array}{l}\text { Cell death; apoptosis; membrane } \\
\text { permeability }\end{array}$ \\
\hline Mukhin et al. (79) & Blade transection & Neuron/glial culture & Single & Single & Severe & Cell death; excitotoxicity \\
\hline Sieg et al. (80) & $\begin{array}{l}\text { Mechanical } \\
\text { compression }\end{array}$ & $\begin{array}{l}\text { Organo-typic cortical } \\
\text { slices }\end{array}$ & Single & Single & Severe & Cell death; apoptosis; axonal damage \\
\hline Slemmer et al. (63) & Cell stretch & Neuronal culture & $\begin{array}{l}\text { Single } \\
\text { Repeat (6) }\end{array}$ & $\begin{array}{l}\text { Single } \\
\text { Six over } 24 \mathrm{~h}\end{array}$ & Mild & Cell viability; cell death \\
\hline
\end{tabular}

Table 7.7: Selected in vivo or ex vivo studies of neurotrauma. 
Chapter Eight

Final Discussion 


\section{SUMMARY}

Traumatic brain injury continues to be a daunting challenge with few available clinical treatment options. Repetitive concussions have been linked to chronic symptoms such as impulsivity, cognitive decline, and motor dysfunction. In order to advance forward towards the development of novel treatments that can prevent these debilitating symptoms it will be imperative to elucidate the mechanisms that link acute injury to chronic neurodegeneration. We have investigated in this dissertation several of these mechanisms that might contribute to disease progression. In particular, we examined blood brain barrier disruption and the role of protein kinase $\mathrm{C}$ activity, endoplasmic reticulum stress and tauopathy, and NADPH oxidative stress and neuronal plasma membrane damage. Once these mechanisms are more fully understood and shown to be similarly upregulated in humans, it will be possible to design targeted therapeutics that can both prevent degenerative changes and slow the progression of chronic traumatic encephalopathy.

The pharmacologic compounds investigated in this dissertation target broad secondary injury cascades. Bryostatin was used historically as a chemotherapy agent with cytotoxic properties. Recent pre-clinical findings have shown its potential for modulating protein kinase $\mathrm{C}$ isozymes within the brain and preventing neuronal injury expansion. We showed it has neuroprotective benefits at the blood brain barrier. Docosahexaenoic acid has been shown to stabilize neuronal membranes, prevent axonal degeneration, and reduce endoplasmic reticulum stress. The multiple therapeutic targets and cost-effective nature of this supplement make it an ideal candidate for clinical trials. We were able to show that it successfully prevented cognitive decline when given after injury in our 
rodent model. Furthermore, lipoic acid is known to readily cross the blood brain barrier and target oxidative stress. We showed that it was effective at reducing NADPH oxidative stress within the neuronal membrane. Further work is needed to determine if it also can prevent mitochondrial related oxidative stress.

\section{FUTURE DIRECTIONS}

Going forward, it will be necessary to utilize the expertise of multi-disciplinary teams. In this dissertation, we highlighted a project that was performed in conjunction with an astronautical engineer and physicist. The collaboration led to an enhanced understanding of the wave mechanisms and how the wave dynamics may be directly causing injury. Similarly, we partnered with Anthony Petraglia, MD to compare two proposed models of chronic traumatic encephalopathy. The collaborative nature of these projects will continue to propel the field further and allow a broader appreciation of different types of TBIs.

We are currently working with a $\mathrm{PhD}$ in mechanical engineering for developing a strategic approach for handling concussion. In the first component, we are investigating strategies for early detection of concussion such as measurements of successive impacts and rotational acceleration. In the second component, we are working on the development of early diagnosis of concussion by blood-based biomarkers. In the third component, we are developing new preventative equipment such as neck sheaths and re-designed helmets. Finally, we will develop a brain health supplement regimen that can be used to prevent cognitive decline following injury.

As mentioned in chapter 7, several unanswered questions are still looming in regards to understanding chronic traumatic encephalopathy development and progression. 
While animal models can give us valuable data about secondary injury cascades, they fail to address the more complex questions regarding genetic predisposition, the association with comorbidities, and the number of impacts or intensity of impacts necessary to push the brain beyond a certain damage threshold. It will be necessary for further human epidemiologic studies to be performed in order to map the progression of the disease. These prospective studies can be used to gather better information about disease prevalence and incidence. The number of concussions is on the rise and the topic of degenerative brain changes is of great importance. This dissertation adds a small but informative piece to the puzzle for our overall understanding degenerative changes following neurotrauma. Future work will investigate how the acute secondary injury cascades work together to contribute to persistent neuroinflammation. Targeting neuroinflammation may be the ideal target for reducing behavioral deficits following injury. 


\section{REFERENCES}

Abdul-Muneer PM, Chandra N, Haorah J. 2014. Interactions of Oxidative Stress and Neurovascular Inflammation in the Pathogenesis of Traumatic Brain Injury. Molecular neurobiology

Abdul-Muneer PM, Schuetz H, Wang F, Skotak M, Jones J, et al. 2013. Induction of oxidative and nitrosative damage leads to cerebrovascular inflammation in an animal model of mild traumatic brain injury induced by primary blast. Free radical biology \& medicine 60: 282-91

Abisambra JF, Jinwal UK, Blair LJ, O'Leary JC, 3rd, Li Q, et al. 2013. Tau accumulation activates the unfolded protein response by impairing endoplasmic reticulumassociated degradation. The Journal of neuroscience : the official journal of the Society for Neuroscience 33: 9498-507

Abisambra JF, Scheff S. 2014. Brain injury in the context of tauopathies. Journal of Alzheimer's disease : JAD 40: 495-518

Acosta SA, Tajiri N, Shinozuka K, Ishikawa H, Grimmig B, et al. 2013. Long-term upregulation of inflammation and suppression of cell proliferation in the brain of adult rats exposed to traumatic brain injury using the controlled cortical impact model. PloS one 8: e53376

Adelson PD, Fellows-Mayle W, Kochanek PM, Dixon CE. 2013. Morris water maze function and histologic characterization of two age-at-injury experimental models of controlled cortical impact in the immature rat. Child's nervous system : ChNS : official journal of the International Society for Pediatric Neurosurgery 29: 43-53

Agarwal A, Sidiq S, Setia S, Bukusoglu E, de Pablo JJ, et al. 2013. Colloid-in-liquid crystal gels that respond to biomolecular interactions. Small 9: 2785-92, 84

Ahmad A, Crupi R, Campolo M, Genovese T, Esposito E, Cuzzocrea S. 2013. Absence of TLR4 reduces neurovascular unit and secondary inflammatory process after traumatic brain injury in mice. PloS one 8: e57208

Allen GV, Gerami D, Esser MJ. 2000. Conditioning effects of repetitive mild neurotrauma on motor function in an animal model of focal brain injury. Neuroscience 99: 93-105

Almli LM, Stevens JS, Smith AK, Kilaru V, Meng Q, et al. 2015. A genome-wide identified risk variant for PTSD is a methylation quantitative trait locus and confers decreased cortical activation to fearful faces. American journal of medical genetics. Part B, Neuropsychiatric genetics : the official publication of the International Society of Psychiatric Genetics 168B: 327-36

Alves JL. 2014. Blood-brain barrier and traumatic brain injury. Journal of neuroscience research 92: 141-7

Amtul Z, Hepburn JD. 2014. Protein markers of cerebrovascular disruption of neurovascular unit: immunohistochemical and imaging approaches. Reviews in the neurosciences

Anderson J, Sandhir R, Hamilton ES, Berman NE. 2009. Impaired expression of neuroprotective molecules in the HIF-1alpha pathway following traumatic brain injury in aged mice. Journal of neurotrauma 26: 1557-66 
Armstead WM, Kiessling JW, Riley J, Cines DB, Higazi AA. 2011. tPA contributes to impaired NMDA cerebrovasodilation after traumatic brain injury through activation of JNK MAPK. Neurological research 33: 726-33

Armstead WM, Riley J, Cines DB, Higazi AA. 2012. Combination therapy with glucagon and a novel plasminogen activator inhibitor-1-derived peptide enhances protection against impaired cerebrovasodilation during hypotension after traumatic brain injury through inhibition of ERK and JNK MAPK. Neurological research 34: 530-7

Arun P, Abu-Taleb R, Oguntayo S, Tanaka M, Wang Y, et al. 2013a. Distinct patterns of expression of traumatic brain injury biomarkers after blast exposure: role of compromised cell membrane integrity. Neuroscience letters 552: 87-91

Arun P, Abu-Taleb R, Valiyaveettil M, Wang Y, Long JB, Nambiar MP. 2013b. Extracellular cyclophilin A protects against blast-induced neuronal injury. Neuroscience research 76: 98-100

Atkins CM, Chen S, Alonso OF, Dietrich WD, Hu BR. 2006. Activation of calcium/calmodulin-dependent protein kinases after traumatic brain injury. Journal of cerebral blood flow and metabolism : official journal of the International Society of Cerebral Blood Flow and Metabolism 26: 1507-18

Aungst SL, Kabadi SV, Thompson SM, Stoica BA, Faden AI. 2014a. Repeated mild traumatic brain injury causes chronic neuroinflammation, changes in hippocampal synaptic plasticity, and associated cognitive deficits. Journal of cerebral blood flow and metabolism : official journal of the International Society of Cerebral Blood Flow and Metabolism

Aungst SL, Kabadi SV, Thompson SM, Stoica BA, Faden AI. 2014b. Repeated mild traumatic brain injury causes chronic neuroinflammation, changes in hippocampal synaptic plasticity, and associated cognitive deficits. Journal of cerebral blood flow and metabolism : official journal of the International Society of Cerebral Blood Flow and Metabolism 34: 1223-32

Avale ME, Rodriguez-Martin T, Gallo JM. 2013. Trans-splicing correction of tau isoform imbalance in a mouse model of tau mis-splicing. Hum Mol Genet 22: 2603-11

Badaut J, Ajao DO, Sorensen DW, Fukuda AM, Pellerin L. 2015. Caveolin expression changes in the neurovascular unit after juvenile traumatic brain injury: signs of blood-brain barrier healing? Neuroscience 285: 215-26

Bailes JE, Dashnaw ML, Petraglia AL, Turner RC. 2014. Cumulative effects of repetitive mild traumatic brain injury. Progress in neurological surgery 28: 50-62

Bailes JE, Patel V. 2014. The potential for DHA to mitigate mild traumatic brain injury. Military medicine 179: 112-6

Bailes JE, Petraglia AL, Omalu BI, Nauman E, Talavage T. 2013. Role of subconcussion in repetitive mild traumatic brain injury. Journal of neurosurgery 119: 1235-45

Bailes JE, Turner RC, Lucke-Wold BP, Patel V, Lee JM. 2015. Chronic Traumatic Encephalopathy: Is It Real? The Relationship Between Neurotrauma and Neurodegeneration. Neurosurgery 62 Suppl 1: 15-24

Bailey ZS, Grinter MB, De La Torre Campos D, VandeVord PJ. 2015a. Blast induced neurotrauma causes overpressure dependent changes to the DNA methylation equilibrium. Neuroscience letters 604: 119-23 
Bailey ZS, Sujith Sajja VS, Hubbard WB, VandeVord PJ. 2015b. Blast Induced Neurotrauma Leads To Changes In The Epigenome. Biomed Sci Instrum 51: 42330

Bales JW, Ma X, Yan HQ, Jenkins LW, Dixon CE. 2010. Expression of protein phosphatase 2B (calcineurin) subunit A isoforms in rat hippocampus after traumatic brain injury. Journal of neurotrauma 27: 109-20

Bandyopadhyay U, Cotney J, Nagy M, Oh S, Leng J, et al. 2013. RNA-Seq profiling of spinal cord motor neurons from a presymptomatic SOD1 ALS mouse. PloS one 8: e53575

Banks SJ, Mayer B, Obuchowski N, Shin W, Lowe M, et al. 2014. Impulsiveness in professional fighters. The Journal of neuropsychiatry and clinical neurosciences 26: $44-50$

Barnes DE, Kaup A, Kirby KA, Byers AL, Diaz-Arrastia R, Yaffe K. 2014. Traumatic brain injury and risk of dementia in older veterans. Neurology 83: 312-9

Barzilai A. 2013. The interrelations between malfunctioning DNA damage response (DDR) and the functionality of the neuro-glio-vascular unit. DNA repair 12: 54357

Bass CR, Panzer MB, Rafaels KA, Wood G, Shridharani J, Capehart B. 2012. Brain injuries from blast. Annals of biomedical engineering 40: 185-202

Baugh CM, Robbins CA, Stern RA, McKee AC. 2014. Current understanding of chronic traumatic encephalopathy. Current treatment options in neurology 16: 306

Baugh CM, Stamm JM, Riley DO, Gavett BE, Shenton ME, et al. 2012. Chronic traumatic encephalopathy: neurodegeneration following repetitive concussive and subconcussive brain trauma. Brain imaging and behavior 6: 244-54

Bedard K, Krause KH. 2007. The NOX family of ROS-generating NADPH oxidases: physiology and pathophysiology. Physiological reviews 87: 245-313

Begum G, Harvey L, Dixon CE, Sun D. 2013. ER stress and effects of DHA as an ER stress inhibitor. Translational stroke research 4: 635-42

Begum G, Yan HQ, Li L, Singh A, Dixon CE, Sun D. 2014. Docosahexaenoic Acid Reduces ER Stress and Abnormal Protein Accumulation and Improves Neuronal Function Following Traumatic Brain Injury. The Journal of neuroscience : the official journal of the Society for Neuroscience 34: 3743-55

Bell KR, Pepping M. 2001. Women and traumatic brain injury. Physical medicine and rehabilitation clinics of North America 12: 169-82

Berkow RL, Schlabach L, Dodson R, Benjamin WH, Jr., Pettit GR, et al. 1993. In vivo administration of the anticancer agent bryostatin 1 activates platelets and neutrophils and modulates protein kinase $\mathrm{C}$ activity. Cancer research 53: 2810-5

Billingsley ML, Kincaid RL. 1997. Regulated phosphorylation and dephosphorylation of tau protein: effects on microtubule interaction, intracellular trafficking and neurodegeneration. The Biochemical journal 323 ( Pt 3): 577-91

Blaylock RL, Maroon J. 2011. Immunoexcitotoxicity as a central mechanism in chronic traumatic encephalopathy-A unifying hypothesis. Surgical neurology international 2: 107

Bolte S, Cordelieres FP. 2006. A guided tour into subcellular colocalization analysis in light microscopy. Journal of microscopy 224: 213-32 
Bolton AN, Saatman KE. 2014. Regional neurodegeneration and gliosis are amplified by mild traumatic brain injury repeated at 24-hour intervals. Journal of neuropathology and experimental neurology 73: 933-47

Booth-Kewley S, Highfill-McRoy RM, Larson GE, Garland CF. 2010. Psychosocial predictors of military misconduct. The Journal of nervous and mental disease 198: 91-8

Borza LR. 2014. A review on the cause-effect relationship between oxidative stress and toxic proteins in the pathogenesis of neurodegenerative diseases. Revista medicochirurgicala a Societatii de Medici si Naturalisti din Iasi 118: 19-27

Boulet T, Kelso ML, Othman SF. 2011. Microscopic magnetic resonance elastography of traumatic brain injury model. Journal of neuroscience methods 201: 296-306

Bower JH, Maraganore DM, Peterson BJ, McDonnell SK, Ahlskog JE, Rocca WA. 2003. Head trauma preceding PD: a case-control study. Neurology 60: 1610-5

Bramlett H, Dietrich WD, 3rd. 2014. Long-Term Consequences of Traumatic Brain Injury: Current Status of Potential Mechanisms of Injury and Neurologic Outcomes. Journal of neurotrauma

Bramlett HM, Dietrich WD. 2001. Neuropathological protection after traumatic brain injury in intact female rats versus males or ovariectomized females. Journal of neurotrauma 18: 891-900

Breunig JJ, Guillot-Sestier MV, Town T. 2013. Brain injury, neuroinflammation and Alzheimer's disease. Frontiers in aging neuroscience 5: 26

Brody DL, Benetatos J, Bennett RE, Klemenhagen KC, Mac Donald CL. 2015. The pathophysiology of repetitive concussive traumatic brain injury in experimental models; new developments and open questions. Molecular and cellular neurosciences

Brown CM, Suzuki S, Jelks KA, Wise PM. 2009. Estradiol is a potent protective, restorative, and trophic factor after brain injury. Seminars in reproductive medicine 27: 240-9

Bruce-Keller AJ, Dimayuga FO, Reed JL, Wang C, Angers R, et al. 2007. Gender and estrogen manipulation do not affect traumatic brain injury in mice. Journal of neurotrauma 24: 203-15

Bruns J, Jr., Hauser WA. 2003. The epidemiology of traumatic brain injury: a review. Epilepsia 44 Suppl 10: 2-10

Budde MD, Shah A, McCrea M, Cullinan WE, Pintar FA, Stemper BD. 2013. Primary blast traumatic brain injury in the rat: relating diffusion tensor imaging and behavior. Frontiers in neurology 4: 154

Carlson SW, Madathil SK, Sama DM, Gao X, Chen J, Saatman KE. 2014. Conditional overexpression of insulin-like growth factor-1 enhances hippocampal neurogenesis and restores immature neuron dendritic processes after traumatic brain injury. Journal of neuropathology and experimental neurology 73: 734-46

Cavallini A, Brewerton S, Bell A, Sargent S, Glover S, et al. 2013. An unbiased approach to identifying tau kinases that phosphorylate tau at sites associated with Alzheimer disease. The Journal of biological chemistry 288: 23331-47

Cernak I. 2015. Blast Injuries and Blast-Induced Neurotrauma: Overview of Pathophysiology and Experimental Knowledge Models and Findings In Brain 
Neurotrauma: Molecular, Neuropsychological, and Rehabilitation Aspects, ed. FHP Kobeissy. Boca Raton (FL)

Chamorro D, Alarcon L, Ponce A, Tapia R, Gonzalez-Aguilar H, et al. 2009.

Phosphorylation of zona occludens- 2 by protein kinase $\mathrm{C}$ epsilon regulates its nuclear exportation. Molecular biology of the cell 20: 4120-9

Chen CH, Shaikenov T, Peterson TR, Aimbetov R, Bissenbaev AK, et al. 2011. ER stress inhibits mTORC2 and Akt signaling through GSK-3beta-mediated phosphorylation of rictor. Sci Signal 4: ra10

Chen S, Atkins CM, Liu CL, Alonso OF, Dietrich WD, Hu BR. 2007. Alterations in mammalian target of rapamycin signaling pathways after traumatic brain injury. Journal of cerebral blood flow and metabolism : official journal of the International Society of Cerebral Blood Flow and Metabolism 27: 939-49

Chen T, Cao L, Dong W, Luo P, Liu W, et al. 2012. Protective effects of mGluR5 positive modulators against traumatic neuronal injury through PKC-dependent activation of MEK/ERK pathway. Neurochemical research 37: 983-90

Chen WJ, Xiong ZA, Zhang M, Yao CG, Zhao ZY, et al. 2013. Picosecond pulsed electric fields induce apoptosis in HeLa cells via the endoplasmic reticulum stress and caspase-dependent signaling pathways. International journal of oncology 42: 963-70

Chen X, Duan XS, Xu LJ, Zhao JJ, She ZF, et al. 2014. Interleukin-10 mediates the neuroprotection of hyperbaric oxygen therapy against traumatic brain injury in mice. Neuroscience 266: 235-43

Cheng ZG, Zhang GD, Shi PQ, Du BS. 2013. Expression and antioxidation of Nrf2/ARE pathway in traumatic brain injury. Asian Pac J Trop Med 6: 305-10

Cho CH. 2011. Frontier of epilepsy research - mTOR signaling pathway. Experimental \& molecular medicine 43: 231-74

Cho HJ, Sajja VS, Vandevord PJ, Lee YW. 2013. Blast induces oxidative stress, inflammation, neuronal loss and subsequent short-term memory impairment in rats. Neuroscience 253: 9-20

Chodobski A, Zink BJ, Szmydynger-Chodobska J. 2011. Blood-brain barrier pathophysiology in traumatic brain injury. Translational stroke research 2: 492516

Choi BY, Jang BG, Kim JH, Lee BE, Sohn M, et al. 2012. Prevention of traumatic brain injury-induced neuronal death by inhibition of NADPH oxidase activation. Brain research 1481: 49-58

Combs CK, Coleman PD, O'Banion MK. 1998. Developmental regulation and PKC dependence of Alzheimer's-type tau phosphorylations in cultured fetal rat hippocampal neurons. Brain research. Developmental brain research 107: 143-58

Conte V, Uryu K, Fujimoto S, Yao Y, Rokach J, et al. 2004. Vitamin E reduces amyloidosis and improves cognitive function in Tg2576 mice following repetitive concussive brain injury. Journal of neurochemistry 90 : 758-64

Corcoran NM, Martin D, Hutter-Paier B, Windisch M, Nguyen T, et al. 2010. Sodium selenate specifically activates PP2A phosphatase, dephosphorylates tau and reverses memory deficits in an Alzheimer's disease model. Journal of clinical neuroscience : official journal of the Neurosurgical Society of Australasia 17: $1025-33$ 
Corsellis JA, Bruton CJ, Freeman-Browne D. 1973. The aftermath of boxing. Psychological medicine 3: 270-303

Cowan CM, Mudher A. 2013. Are tau aggregates toxic or protective in tauopathies? Frontiers in neurology 4: 114

Creeley CE, Wozniak DF, Bayly PV, Olney JW, Lewis LM. 2004. Multiple episodes of mild traumatic brain injury result in impaired cognitive performance in mice. Academic emergency medicine : official journal of the Society for Academic Emergency Medicine 11: 809-19

Cullinan SB, Diehl JA. 2006. Coordination of ER and oxidative stress signaling: the PERK/Nrf2 signaling pathway. The international journal of biochemistry \& cell biology 38: 317-32

Curtis L, Epstein P. 2014. Nutritional treatment for acute and chronic traumatic brain injury patients. Journal of neurosurgical sciences 58: 151-60

Dai SS, Zhou YG, Li W, An JH, Li P, et al. 2010. Local glutamate level dictates adenosine A2A receptor regulation of neuroinflammation and traumatic brain injury. The Journal of neuroscience : the official journal of the Society for Neuroscience 30: 5802-10

Dapul HR, Park J, Zhang J, Lee C, DanEshmand A, et al. 2013. Concussive injury before or after controlled cortical impact exacerbates histopathology and functional outcome in a mixed traumatic brain injury model in mice. Journal of neurotrauma 30: 382-91

Dash PK, Johnson D, Clark J, Orsi SA, Zhang M, et al. 2011. Involvement of the glycogen synthase kinase-3 signaling pathway in TBI pathology and neurocognitive outcome. PloS one 6: e24648

Day NL, Floyd CL, D'Alessandro TL, Hubbard WJ, Chaudry IH. 2013. 17beta-estradiol confers protection after traumatic brain injury in the rat and involves activation of $\mathrm{G}$ protein-coupled estrogen receptor 1. Journal of neurotrauma 30: 1531-41

De Bock M, Decrock E, Wang N, Bol M, Vinken M, et al. 2014. The dual face of connexin-based astroglial Ca communication: A key player in brain physiology and a prime target in pathology. Biochimica et biophysica acta

de Calignon A, Polydoro M, Suarez-Calvet M, William C, Adamowicz DH, et al. 2012. Propagation of tau pathology in a model of early Alzheimer's disease. Neuron 73: 685-97

DeFord SM, Wilson MS, Rice AC, Clausen T, Rice LK, et al. 2002. Repeated mild brain injuries result in cognitive impairment in $\mathrm{B} 6 \mathrm{C} 3 \mathrm{~F} 1$ mice. Journal of neurotrauma 19: 427-38

DeKosky ST, Blennow K, Ikonomovic MD, Gandy S. 2013. Acute and chronic traumatic encephalopathies: pathogenesis and biomarkers. Nature reviews. Neurology 9: 192-200

Deng Y, Xiong Z, Chen P, Wei J, Chen S, Yan Z. 2014. beta-amyloid impairs the regulation of N-methyl-D-aspartate receptors by glycogen synthase kinase 3 . Neurobiology of aging 35: 449-59

DeRoss AL, Adams JE, Vane DW, Russell SJ, Terella AM, Wald SL. 2002. Multiple head injuries in rats: effects on behavior. The Journal of trauma 52: 708-14

Dinapoli VA, Benkovic SA, Li X, Kelly KA, Miller DB, et al. 2010. Age exaggerates proinflammatory cytokine signaling and truncates signal transducers and 
activators of transcription 3 signaling following ischemic stroke in the rat. Neuroscience 170: 633-44

Ding K, Wang H, Xu J, Li T, Zhang L, et al. 2014. Melatonin stimulates antioxidant enzymes and reduces oxidative stress in experimental traumatic brain injury: the Nrf2-ARE signaling pathway as a potential mechanism. Free radical biology \& medicine 73: 1-11

Dobrowolska JA, Gibson CJ. Society for Neuroscience2005.

Donovan V, Bianchi A, Hartman R, Bhanu B, Carson MJ, Obenaus A. 2012.

Computational analysis reveals increased blood deposition following repeated mild traumatic brain injury. NeuroImage. Clinical 1: 18-28

Donovan V, Kim C, Anugerah AK, Coats JS, Oyoyo U, et al. 2014. Repeated mild traumatic brain injury results in long-term white-matter disruption. Journal of cerebral blood flow and metabolism : official journal of the International Society of Cerebral Blood Flow and Metabolism 34: 715-23

Dubrovsky AS, Friedman D, Kocilowicz H. 2014. Pediatric post-traumatic headaches and peripheral nerve blocks of the scalp: a case series and patient satisfaction survey. Headache 54: 878-87

Eckert A, Nisbet R, Grimm A, Gotz J. 2013. March separate, strike together - Role of phosphorylated TAU in mitochondrial dysfunction in Alzheimer's disease. Biochimica et biophysica acta

Effgen GB, Vogel EW, 3rd, Lynch KA, Lobel A, Hue CD, et al. 2014. Isolated primary blast alters neuronal function with minimal cell death in organotypic hippocampal slice cultures. Journal of neurotrauma 31: 1202-10

Ekinci FJ, Shea TB. 1997. Selective activation by bryostatin-1 demonstrates unique roles for PKC epsilon in neurite extension and tau phosphorylation. International journal of developmental neuroscience : the official journal of the International Society for Developmental Neuroscience 15: 867-74

Ellenbogen RG, Berger MS, Batjer HH. 2010. The National Football League and concussion: leading a culture change in contact sports. World neurosurgery 74 : 560-5

Farace E, Alves WM. 2000a. Do women fare worse: a metaanalysis of gender differences in traumatic brain injury outcome. Journal of neurosurgery 93: 539-45

Farace E, Alves WM. 2000b. Do women fare worse? A metaanalysis of gender differences in outcome after traumatic brain injury. Neurosurgical focus 8: e6

Farook JM, Shields J, Tawfik A, Markand S, Sen T, et al. 2013. GADD34 induces cell death through inactivation of Akt following traumatic brain injury. Cell death \& disease 4: e754

Feng S, Li D, Li Y, Yang X, Han S, Li J. 2013. Insight into hypoxic preconditioning and ischemic injury through determination of nPKCepsilon-interacting proteins in mouse brain. Neurochemistry international 63: 69-79

Ferguson S, Mouzon B, Kayihan G, Wood M, Poon F, et al. 2010. Apolipoprotein E genotype and oxidative stress response to traumatic brain injury. Neuroscience 168: $811-9$

Ferreira AP, Rodrigues FS, Della-Pace ID, Mota BC, Oliveira SM, et al. 2013. The effect of NADPH-oxidase inhibitor apocynin on cognitive impairment induced by 
moderate lateral fluid percussion injury: Role of inflammatory and oxidative brain damage. Neurochemistry international 63: 583-93

Fonseca AC, Ferreiro E, Oliveira CR, Cardoso SM, Pereira CF. 2013. Activation of the endoplasmic reticulum stress response by the amyloid-beta 1-40 peptide in brain endothelial cells. Biochimica et biophysica acta 1832: 2191-203

Freeman LC, Ting JP. 2015. The pathogenic role of the inflammasome in neurodegenerative diseases. Journal of neurochemistry

Freund Levi Y, Vedin I, Cederholm T, Basun H, Faxen Irving G, et al. 2014. Transfer of omega-3 fatty acids across the blood-brain barrier after dietary supplementation with a docosahexaenoic acid-rich omega-3 fatty acid preparation in patients with Alzheimer's disease: the OmegAD study. Journal of internal medicine 275: 42836

Friess SH, Ichord RN, Ralston J, Ryall K, Helfaer MA, et al. 2009. Repeated traumatic brain injury affects composite cognitive function in piglets. Journal of neurotrauma 26: 1111-21

Fujita M, Wei EP, Povlishock JT. 2012. Intensity- and interval-specific repetitive traumatic brain injury can evoke both axonal and microvascular damage. Journal of neurotrauma 29: 2172-80

Gandy S, DeKosky ST. 2014. [18F]-T807 tauopathy PET imaging in chronic traumatic encephalopathy. F1000Research 3: 229

Ganpule S, Alai A, Plougonven E, Chandra N. 2013. Mechanics of blast loading on the head models in the study of traumatic brain injury using experimental and computational approaches. Biomech Model Mechanobiol 12: 511-31

Gao HM, Zhou H, Hong JS. 2012. NADPH oxidases: novel therapeutic targets for neurodegenerative diseases. Trends in pharmacological sciences 33: 295-303

Garringer JA, Niyonkuru C, McCullough EH, Loucks T, Dixon CE, et al. 2013. Impact of aromatase genetic variation on hormone levels and global outcome after severe TBI. Journal of neurotrauma 30: 1415-25

Gatson JW, Liu MM, Abdelfattah K, Wigginton JG, Smith S, et al. 2012. Estrone is neuroprotective in rats after traumatic brain injury. Journal of neurotrauma 29: 2209-19

Gavett BE, Cantu RC, Shenton M, Lin AP, Nowinski CJ, et al. 2011a. Clinical appraisal of chronic traumatic encephalopathy: current perspectives and future directions. Current opinion in neurology 24: 525-31

Gavett BE, Stern RA, McKee AC. 2011b. Chronic traumatic encephalopathy: a potential late effect of sport-related concussive and subconcussive head trauma. Clinics in sports medicine 30: 179-88, xi

Geddes-Klein DM, Serbest G, Mesfin MN, Cohen AS, Meaney DF. 2006. Pharmacologically induced calcium oscillations protect neurons from increases in cytosolic calcium after trauma. Journal of neurochemistry 97: 462-74

Genovese RF, Simmons LP, Ahlers ST, Maudlin-Jeronimo E, Dave JR, Boutte AM. 2013. Effects of mild TBI from repeated blast overpressure on the expression and extinction of conditioned fear in rats. Neuroscience 254: 120-9

Ghadiri T, Sharifzadeh M, Khodagholi F, Modarres Mousavi SM, Hassanzadeh G, et al. 2014. A novel traumatic brain injury model for induction of mild brain injury in rats. Journal of neuroscience methods 233: 18-27 
Giordano G, Sanchez-Perez AM, Burgal M, Montoliu C, Costa LG, Felipo V. 2005. Chronic exposure to ammonia induces isoform-selective alterations in the intracellular distribution and NMDA receptor-mediated translocation of protein kinase $C$ in cerebellar neurons in culture. Journal of neurochemistry 92: 143-57

Giraldo E, Lloret A, Fuchsberger T, Vina J. 2014. Abeta and tau toxicities in Alzheimer's are linked via oxidative stress-induced p38 activation: protective role of vitamin E. Redox biology 2: 873-7

Girolamo F, Dallatomasina A, Rizzi M, Errede M, Walchli T, et al. 2013. Diversified expression of NG2/CSPG4 isoforms in glioblastoma and human foetal brain identifies pericyte subsets. PloS one 8: e84883

Glushakova OY, Johnson D, Hayes RL. 2014. Delayed Increases in Microvascular Pathology after Experimental Traumatic Brain Injury Are Associated with Prolonged Inflammation, Blood-Brain Barrier Disruption, and Progressive White Matter Damage. Journal of neurotrauma

Goddeyne C, Nichols J, Wu C, Anderson T. 2015. Repetitive mild traumatic brain injury induces ventriculomegaly and cortical thinning in juvenile rats. Journal of neurophysiology 113: 3268-80

Goldstein LE, Fisher AM, Tagge CA, Zhang XL, Velisek L, et al. 2012. Chronic traumatic encephalopathy in blast-exposed military veterans and a blast neurotrauma mouse model. Science translational medicine 4: 134ra60

Gomes MB, Negrato CA. 2014. Alpha-lipoic acid as a pleiotropic compound with potential therapeutic use in diabetes and other chronic diseases. Diabetology \& metabolic syndrome 6: 80

Goncalves A, Leal E, Paiva A, Teixeira Lemos E, Teixeira F, et al. 2012. Protective effects of the dipeptidyl peptidase IV inhibitor sitagliptin in the blood-retinal barrier in a type 2 diabetes animal model. Diabetes, obesity \& metabolism 14: 454-63

Gong T, Wang Q, Lin Z, Chen ML, Sun GZ. 2012. Endoplasmic reticulum (ER) stress inhibitor salubrinal protects against ceramide-induced SH-SY5Y cell death. Biochemical and biophysical research communications 427: 461-5

Gozzelino R, Jeney V, Soares MP. 2010. Mechanisms of cell protection by heme oxygenase-1. Annual review of pharmacology and toxicology 50: 323-54

Green KN, Martinez-Coria H, Khashwji H, Hall EB, Yurko-Mauro KA, et al. 2007. Dietary docosahexaenoic acid and docosapentaenoic acid ameliorate amyloid-beta and tau pathology via a mechanism involving presenilin 1 levels. The Journal of neuroscience : the official journal of the Society for Neuroscience 27: 4385-95

Guo D, Standley C, Bellve K, Fogarty K, Bao ZZ. 2012. Protein kinase Calpha and integrin-linked kinase mediate the negative axon guidance effects of Sonic hedgehog. Molecular and cellular neurosciences 50: 82-92

Gupta RK, Przekwas A. 2013. Mathematical Models of Blast-Induced TBI: Current Status, Challenges, and Prospects. Frontiers in neurology 4: 59

Guskiewicz KM, Marshall SW, Bailes J, McCrea M, Cantu RC, et al. 2005. Association between recurrent concussion and late-life cognitive impairment in retired professional football players. Neurosurgery 57: 719-26; discussion 19-26 
Hagberg H, Wilson MA, Matsushita H, Zhu C, Lange M, et al. 2004. PARP-1 gene disruption in mice preferentially protects males from perinatal brain injury. Journal of neurochemistry 90: 1068-75

Haghighi F, Ge Y, Chen S, Xin Y, Umali MU, et al. 2015. Neuronal DNA Methylation Profiling of Blast-related Traumatic Brain Injury (TBI). Journal of neurotrauma

Hall ED, Vaishnav RA, Mustafa AG. 2010. Antioxidant therapies for traumatic brain injury. Neurotherapeutics : the journal of the American Society for Experimental NeuroTherapeutics 7: 51-61

Hamm RJ, Jenkins LW, Lyeth BG, White-Gbadebo DM, Hayes RL. 1991. The effect of age on outcome following traumatic brain injury in rats. Journal of neurosurgery 75: 916-21

Hasadsri L, Wang BH, Lee JV, Erdman JW, Llano DA, et al. 2013. Omega-3 fatty acids as a putative treatment for traumatic brain injury. Journal of neurotrauma 30 : 897-906

Hawkins BE, Krishnamurthy S, Castillo-Carranza DL, Sengupta U, Prough DS, et al. 2013. Rapid accumulation of endogenous tau oligomers in a rat model of traumatic brain injury: possible link between traumatic brain injury and sporadic tauopathies. The Journal of biological chemistry 288: 17042-50

Hebron ML, Lonskaya I, Sharpe K, Weerasinghe PP, Algarzae NK, et al. 2013. Parkin ubiquitinates Tar-DNA binding protein-43 (TDP-43) and promotes its cytosolic accumulation via interaction with histone deacetylase 6 (HDAC6). The Journal of biological chemistry 288: 4103-15

Hernandez-Ontiveros DG, Tajiri N, Acosta S, Giunta B, Tan J, Borlongan CV. 2013. Microglia activation as a biomarker for traumatic brain injury. Frontiers in neurology 4: 30

Hetz C, Mollereau B. 2014. Disturbance of endoplasmic reticulum proteostasis in neurodegenerative diseases. Nature reviews. Neuroscience 15: 233-49

Hilton GD, Stoica BA, Byrnes KR, Faden AI. 2008. Roscovitine reduces neuronal loss, glial activation, and neurologic deficits after brain trauma. Journal of cerebral blood flow and metabolism : official journal of the International Society of Cerebral Blood Flow and Metabolism 28: 1845-59

Hiramatsu N, Messah C, Han J, Lavail MM, Kaufman RJ, Lin JH. 2014. Translational and Post-Translational Regulation of XIAP by eIF2alpha and ATF4 promotes ER stress-induced Cell Death during the Unfolded Protein Response. Molecular biology of the cell

Ho YH, Lin YT, Wu CW, Chao YM, Chang AY, Chan JY. 2015. Peripheral inflammation increases seizure susceptibility via the induction of neuroinflammation and oxidative stress in the hippocampus. J Biomed Sci 22: 46

Hoane MR, Lasley LA, Akstulewicz SL. 2004. Middle age increases tissue vulnerability and impairs sensorimotor and cognitive recovery following traumatic brain injury in the rat. Behavioural brain research 153: 189-97

Hong MY, Gao JL, Cui JZ, Wang KJ, Tian YX, et al. 2012. Effect of c-Jun NH(2)terminal kinase-mediated p53 expression on neuron autophagy following traumatic brain injury in rats. Chin Med J (Engl) 125: 2019-24

Hongpaisan J, Sun MK, Alkon DL. 2011. PKC epsilon activation prevents synaptic loss, Abeta elevation, and cognitive deficits in Alzheimer's disease transgenic mice. 
The Journal of neuroscience : the official journal of the Society for Neuroscience 31: $630-43$

Hoozemans JJ, Scheper W. 2012. Endoplasmic reticulum: the unfolded protein response is tangled in neurodegeneration. The international journal of biochemistry \& cell biology 44: 1295-8

Hoozemans JJ, van Haastert ES, Nijholt DA, Rozemuller AJ, Eikelenboom P, Scheper W. 2009. The unfolded protein response is activated in pretangle neurons in Alzheimer's disease hippocampus. The American journal of pathology 174: 124151

Hu SL, Hu R, Li F, Liu Z, Xia YZ, et al. 2008. Hyperbaric oxygen preconditioning protects against traumatic brain injury at high altitude. Acta neurochirurgica. Supplement 105: 191-6

Hua Y, Lin S, Gu L. 2015. Relevance of Blood Vessel Networks in Blast-Induced Traumatic Brain Injury. Comput Math Methods Med 2015: 928236

Huang H, Ayoub J. 2008. Applicability of the Forchheimer equation for non-Darcy flow in porous media. Spe $J$ 13: 112-22

Huang L, Wan J, Chen Y, Wang Z, Hui L, et al. 2013. Inhibitory effects of p38 inhibitor against mitochondrial dysfunction in the early brain injury after subarachnoid hemorrhage in mice. Brain research 1517: 133-40

Huber BR, Meabon JS, Martin TJ, Mourad PD, Bennett R, et al. 2013. Blast exposure causes early and persistent aberrant phospho- and cleaved-tau expression in a murine model of mild blast-induced traumatic brain injury. Journal of Alzheimer's disease : JAD 37: 309-23

Hue CD, Cao S, Dale Bass CR, Meaney DF, Morrison B, 3rd. 2014. Repeated Primary Blast Injury Causes Delayed Recovery, but not Additive Disruption, in an In Vitro Blood-Brain Barrier Model. Journal of neurotrauma 31: 951-60

Hwang J, Hwang H, Lee HW, Suk K. 2010. Microglia signaling as a target of donepezil. Neuropharmacology 58: 1122-9

Iliff JJ, Chen MJ, Plog BA, Zeppenfeld DM, Soltero M, et al. 2014. Impairment of glymphatic pathway function promotes tau pathology after traumatic brain injury. The Journal of neuroscience : the official journal of the Society for Neuroscience 34: 16180-93

Indharty RS. 2013. The increase of serum Bcl-2 concentration in moderate head injury outcome: The role of ACTH4-10Pro(8)-Gly(9)-Pro(10.). Asian journal of neurosurgery 8: 83-9

Iqbal K, Gong CX, Liu F. 2013. Hyperphosphorylation-induced tau oligomers. Frontiers in neurology 4: 112

James LM, Strom TQ, Leskela J. 2014. Risk-taking behaviors and impulsivity among veterans with and without PTSD and mild TBI. Military medicine 179: 357-63

Jean A, Nyein MK, Zheng JQ, Moore DF, Joannopoulos JD, Radovitzky R. 2014. An animal-to-human scaling law for blast-induced traumatic brain injury risk assessment. Proceedings of the National Academy of Sciences of the United States of America 111: 15310-5

Jia J, Guan D, Zhu W, Alkayed NJ, Wang MM, et al. 2009. Estrogen inhibits Fasmediated apoptosis in experimental stroke. Experimental neurology 215: 48-52 
Johnson EM, Traver KL, Hoffman SW, Harrison CR, Herman JP. 2013. Environmental enrichment protects against functional deficits caused by traumatic brain injury. Frontiers in behavioral neuroscience 7: 44

Johnson SM, Torrice CD, Bell JF, Monahan KB, Jiang Q, et al. 2010a. Mitigation of hematologic radiation toxicity in mice through pharmacological quiescence induced by CDK4/6 inhibition. The Journal of clinical investigation 120: 2528-36

Johnson VE, Stewart W, Smith DH. 2010b. Traumatic brain injury and amyloid-beta pathology: a link to Alzheimer's disease? Nature reviews. Neuroscience 11:36170

Kabadi SV, Stoica BA, Byrnes KR, Hanscom M, Loane DJ, Faden AI. 2012a. Selective CDK inhibitor limits neuroinflammation and progressive neurodegeneration after brain trauma. Journal of cerebral blood flow and metabolism : official journal of the International Society of Cerebral Blood Flow and Metabolism 32: 137-49

Kabadi SV, Stoica BA, Hanscom M, Loane DJ, Kharebava G, et al. 2012b. CR8, a selective and potent CDK inhibitor, provides neuroprotection in experimental traumatic brain injury. Neurotherapeutics : the journal of the American Society for Experimental NeuroTherapeutics 9: 405-21

Kadyan V, Mysiw WJ, Bogner JA, Corrigan JD, Fugate LP, Clinchot DM. 2004. Gender differences in agitation after traumatic brain injury. American journal of physical medicine \& rehabilitation / Association of Academic Physiatrists 83: 747-52

Kamat PK, Rai S, Swarnkar S, Shukla R, Nath C. 2014. Molecular and Cellular Mechanism of Okadaic Acid (OKA)-Induced Neurotoxicity: A Novel Tool for Alzheimer's Disease Therapeutic Application. Molecular neurobiology

Kanayama G, Takeda M, Niigawa H, Ikura Y, Tamii H, et al. 1996. The effects of repetitive mild brain injury on cytoskeletal protein and behavior. Methods and findings in experimental and clinical pharmacology 18: 105-15

Kane MJ, Angoa-Perez M, Briggs DI, Viano DC, Kreipke CW, Kuhn DM. 2012. A mouse model of human repetitive mild traumatic brain injury. Journal of neuroscience methods 203: 41-9

Kanemaru K, Takio K, Miura R, Titani K, Ihara Y. 1992. Fetal-type phosphorylation of the tau in paired helical filaments. Journal of neurochemistry 58: 1667-75

Kang EB, Kwon IS, Koo JH, Kim EJ, Kim CH, et al. 2013. Treadmill exercise represses neuronal cell death and inflammation during Abeta-induced ER stress by regulating unfolded protein response in aged presenilin 2 mutant mice. Apoptosis : an international journal on programmed cell death 18: 1332-47

Kang JH, Asai D, Yamada S, Toita R, Oishi J, et al. 2008. A short peptide is a protein kinase C (PKC) alpha-specific substrate. Proteomics 8: 2006-11

Karasu A, Aras Y, Sabanci PA, Saglam G, Izgi N, et al. 2010. The effects of protein kinase $\mathrm{C}$ activator phorbol dibutyrate on traumatic brain edema and aquaporin-4 expression. Ulusal travma ve acil cerrahi dergisi $=$ Turkish journal of trauma \& emergency surgery: TJTES 16: 390-4

Kasturi BS, Stein DG. 2009. Progesterone decreases cortical and sub-cortical edema in young and aged ovariectomized rats with brain injury. Restorative neurology and neuroscience 27: $265-75$ 
Kates SA, Lader AS, Casale R, Beeuwkes R, 3rd. 2014. Pre-clinical and Clinical Safety Studies of CMX-2043: A Cytoprotective Lipoic Acid Analogue for IschaemiaReperfusion Injury. Basic \& clinical pharmacology \& toxicology

Kaufman RJ, Malhotra JD. 2014. Calcium trafficking integrates endoplasmic reticulum function with mitochondrial bioenergetics. Biochimica et biophysica acta

Ke C, Poon WS, Ng HK, Pang JC, Chan Y. 2001. Heterogeneous responses of aquaporin4 in oedema formation in a replicated severe traumatic brain injury model in rats. Neuroscience letters 301: 21-4

Kester MI, Goos JD, Teunissen CE, Benedictus MR, Bouwman FH, et al. 2014. Associations Between Cerebral Small-Vessel Disease and Alzheimer Disease Pathology as Measured by Cerebrospinal Fluid Biomarkers. JAMA neurology

Khan F, Baguley IJ, Cameron ID. 2003. 4: Rehabilitation after traumatic brain injury. Med J Aust 178: 290-5

Kim H, Han SH, Quan HY, Jung YJ, An J, et al. 2012a. Bryostatin-1 promotes long-term potentiation via activation of PKCalpha and PKCepsilon in the hippocampus. Neuroscience 226: 348-55

Kim MH, Jung YS, Moon CH, Jeong EM, Lee SH, et al. 2003. Isoform-specific induction of PKC-epsilon by high glucose protects heart-derived H9c2 cells against hypoxic injury. Biochemical and biophysical research communications 309: 1-6

Kim P, Choi I, Pena IC, Kim HJ, Kwon KJ, et al. 2012b. A simple behavioral paradigm to measure impulsive behavior in an animal model of attention deficit hyperactivity disorder (ADHD) of the spontaneously hypertensive rats. Biomolecules \& therapeutics 20: 125-31

Kim YA, Park SL, Kim MY, Lee SH, Baik EJ, et al. 2010. Role of PKCbetaII and PKCdelta in blood-brain barrier permeability during aglycemic hypoxia. Neuroscience letters 468: 254-8

King MK, Pardo M, Cheng Y, Downey K, Jope RS, Beurel E. 2014. Glycogen synthase kinase-3 inhibitors: Rescuers of cognitive impairments. Pharmacology \& therapeutics 141: 1-12

Kizub IV, Klymenko KI, Soloviev AI. 2014. Protein kinase C in enhanced vascular tone in diabetes mellitus. International journal of cardiology 174: 230-42

Klemenhagen KC, O'Brien SP, Brody DL. 2013. Repetitive concussive traumatic brain injury interacts with post-injury foot shock stress to worsen social and depressionlike behavior in mice. PloS one 8: e74510

Kobeissy F, Mondello S, Tumer N, Toklu HZ, Whidden MA, et al. 2013. Assessing neuro-systemic \& behavioral components in the pathophysiology of blast-related brain injury. Frontiers in neurology 4: 186

Kochanek PM, Dixon CE, Shellington DK, Shin SS, Bayir H, et al. 2013. Screening of biochemical and molecular mechanisms of secondary injury and repair in the brain after experimental blast-induced traumatic brain injury in rats. Journal of neurotrauma 30: 920-37

Koliatsos VE, Xu L. 2015. The Problem of Neurodegeneration in Cumulative Sports Concussions: Emphasis on Neurofibrillary Tangle Formation In Brain Neurotrauma: Molecular, Neuropsychological, and Rehabilitation Aspects, ed. FHP Kobeissy. Boca Raton (FL) 
Kondo A, Shahpasand K, Mannix R, Qiu J, Moncaster J, et al. 2015. Antibody against early driver of neurodegeneration cis P-tau blocks brain injury and tauopathy. Nature 523: 431-6

Kors EE, Terwindt GM, Vermeulen FL, Fitzsimons RB, Jardine PE, et al. 2001. Delayed cerebral edema and fatal coma after minor head trauma: role of the CACNA1A calcium channel subunit gene and relationship with familial hemiplegic migraine. Annals of neurology 49: 753-60

Kou Z, Vandevord PJ. 2014. Traumatic white matter injury and glial activation: From basic science to clinics. Glia

Kovesdi E, Kamnaksh A, Wingo D, Ahmed F, Grunberg NE, et al. 2012. Acute minocycline treatment mitigates the symptoms of mild blast-induced traumatic brain injury. Frontiers in neurology 3: 111

Krajewska M, Xu L, Xu W, Krajewski S, Kress CL, et al. 2011. Endoplasmic reticulum protein BI-1 modulates unfolded protein response signaling and protects against stroke and traumatic brain injury. Brain research 1370: 227-37

Kramerov AA, Golub AG, Bdzhola VG, Yarmoluk SM, Ahmed K, et al. 2011. Treatment of cultured human astrocytes and vascular endothelial cells with protein kinase CK2 inhibitors induces early changes in cell shape and cytoskeleton. Mol Cell Biochem 349: 125-37

Ksiezak-Reding H, Pyo HK, Feinstein B, Pasinetti GM. 2003. Akt/PKB kinase phosphorylates separately Thr212 and Ser214 of tau protein in vitro. Biochimica et biophysica acta 1639: 159-68

Kumar A, Stoica BA, Sabirzhanov B, Burns MP, Faden AI, Loane DJ. 2013. Traumatic brain injury in aged animals increases lesion size and chronically alters microglial/macrophage classical and alternative activation states. Neurobiology of aging 34: 1397-411

Kuo CL, Ho FM, Chang MY, Prakash E, Lin WW. 2008. Inhibition of lipopolysaccharide-induced inducible nitric oxide synthase and cyclooxygenase-2 gene expression by 5-aminoimidazole-4-carboxamide riboside is independent of AMP-activated protein kinase. J Cell Biochem 103: 931-40

Kuo JR, Cheng YH, Chen YS, Chio CC, Gean PW. 2013. Involvement of extracellular signal regulated kinases in traumatic brain injury-induced depression in rodents. Journal of neurotrauma 30: 1223-31

Lai AY, Dibal CD, Armitage GA, Winship IR, Todd KG. 2013. Distinct activation profiles in microglia of different ages: a systematic study in isolated embryonic to aged microglial cultures. Neuroscience 254: 185-95

Lakis N, Corona RJ, Toshkezi G, Chin LS. 2013. Chronic traumatic encephalopathy neuropathology in athletes and war veterans. Neurological research 35: 290-9

Lallemend F, Hadjab S, Hans G, Moonen G, Lefebvre PP, Malgrange B. 2005. Activation of protein kinase CbetaI constitutes a new neurotrophic pathway for deafferented spiral ganglion neurons. Journal of cell science 118: 4511-25

Lambrecht C, Haesen D, Sents W, Ivanova E, Janssens V. 2013. Structure, regulation, and pharmacological modulation of PP2A phosphatases. Methods in molecular biology 1053: 283-305

Lanz TV, Becker S, Osswald M, Bittner S, Schuhmann MK, et al. 2013. Protein kinase Cbeta as a therapeutic target stabilizing blood-brain barrier disruption in 
experimental autoimmune encephalomyelitis. Proceedings of the National Academy of Sciences of the United States of America 110: 14735-40

LaPlaca MC, Cullen DK, McLoughlin JJ, Cargill RS, 2nd. 2005. High rate shear strain of three-dimensional neural cell cultures: a new in vitro traumatic brain injury model. Journal of biomechanics 38: 1093-105

LaPlaca MC, Thibault LE. 1998. Dynamic mechanical deformation of neurons triggers an acute calcium response and cell injury involving the N-methyl-D-aspartate glutamate receptor. Journal of neuroscience research 52: 220-9

Larner SF, Hayes RL, McKinsey DM, Pike BR, Wang KK. 2004. Increased expression and processing of caspase-12 after traumatic brain injury in rats. Journal of neurochemistry 88: 78-90

Laskowitz DT, Dawson HN. 2014. Genetic influences and neuropathological sequelae of repetitive brain injury. Annals of neurology 75: 617-8

Laurer HL, Bareyre FM, Lee VM, Trojanowski JQ, Longhi L, et al. 2001. Mild head injury increasing the brain's vulnerability to a second concussive impact. Journal of neurosurgery 95: 859-70

Le MN, Kim W, Lee S, McKee AC, Hall GF. 2012. Multiple mechanisms of extracellular tau spreading in a non-transgenic tauopathy model. American journal of neurodegenerative disease 1: 316-33

Lebbadi M, Julien C, Phivilay A, Tremblay C, Emond V, et al. 2011. Endogenous conversion of omega- 6 into omega-3 fatty acids improves neuropathology in an animal model of Alzheimer's disease. Journal of Alzheimer's disease : JAD 27: 853-69

Leeds PR, Yu F, Wang Z, Chiu CT, Zhang Y, et al. 2014. A New Avenue for Lithium: Intervention in Traumatic Brain Injury. ACS chemical neuroscience

Li A, Zou F, Fu H, Cui G, Yan Y, et al. 2013a. Upregulation of CRM1 relates to neuronal apoptosis after traumatic brain injury in adult rats. Journal of molecular neuroscience : $M N$ 51: 208-18

Li H, Wang Y, Feng D, Liu Y, Xu M, et al. 2014a. Alterations in the time course of expression of the Nox family in the brain in a rat experimental cerebral ischemia and reperfusion model: effects of melatonin. Journal of pineal research 57: 110-9

Li J, Siegel M, Yuan M, Zeng Z, Finnucan L, et al. 2011. Estrogen enhances neurogenesis and behavioral recovery after stroke. Journal of cerebral blood flow and metabolism : official journal of the International Society of Cerebral Blood Flow and Metabolism 31: 413-25

Li Z, Tian F, Shao Z, Shen X, Qi X, et al. 2014b. Expression and clinical significance of non-phagocytic cell oxidase 2 and 4 after human traumatic brain injury. Neurological sciences : official journal of the Italian Neurological Society and of the Italian Society of Clinical Neurophysiology

Li Z, Zhao L, Hang H, Zhu N, Ning B, Lv Z. 2013b. Spatiotemporal patterns and essential role of MSK1 expression after rat spinal cord injury. Neurochemical research 38: 2581-7

Lim CS, Alkon DL. 2014. PKCepsilon promotes HuD-mediated neprilysin mRNA stability and enhances neprilysin-induced Abeta degradation in brain neurons. PloS one 9: e97756 
Lin HW, Della-Morte D, Thompson JW, Gresia VL, Narayanan SV, et al. 2012. Differential effects of delta and epsilon protein kinase $\mathrm{C}$ in modulation of postischemic cerebral blood flow. Advances in experimental medicine and biology 737: 63-9

Lipsky RH, Lin M. 2015. Genetic predictors of outcome following traumatic brain injury. Handbook of clinical neurology 127: 23-41

Liu MC, Kobeissy F, Zheng W, Zhang Z, Hayes RL, Wang KK. 2011. Dual vulnerability of tau to calpains and caspase-3 proteolysis under neurotoxic and neurodegenerative conditions. ASN neuro 3: e00051

Liu W, Wang P, Shang C, Chen L, Cai H, et al. 2014a. Endophilin-1 regulates bloodbrain barrier permeability by controlling ZO-1 and occludin expression via the EGFR-ERK1/2 pathway. Brain research

Liu X, Zhao S, Liu F, Kang J, Xiao A, et al. 2014b. Remote Ischemic Postconditioning Alleviates Cerebral Ischemic Injury by Attenuating Endoplasmic Reticulum Stress-Mediated Apoptosis. Translational stroke research

Liu XA, Liao K, Liu R, Wang HH, Zhang Y, et al. 2010. Tau dephosphorylation potentiates apoptosis by mechanisms involving a failed dephosphorylation/activation of Bcl-2. Journal of Alzheimer's disease : JAD 19: 953-62

Liu Y, Su Y, Wang J, Sun S, Wang T, et al. 2013. Rapamycin decreases tau phosphorylation at Ser214 through regulation of cAMP-dependent kinase. Neurochemistry international 62: 458-67

Liu ZC, Fu ZQ, Song J, Zhang JY, Wei YP, et al. 2012. Bip enhanced the association of GSK-3beta with tau during ER stress both in vivo and in vitro. Journal of Alzheimer's disease : JAD 29: 727-40

Loane DJ, Kumar A. 2015. Microglia in the TBI brain: The good, the bad, and the dysregulated. Experimental neurology

Loane DJ, Stoica BA, Byrnes KR, Jeong W, Faden AI. 2013. Activation of mGluR5 and inhibition of NADPH oxidase improves functional recovery after traumatic brain injury. Journal of neurotrauma 30: 403-12

Lobo V, Patil A, Phatak A, Chandra N. 2010. Free radicals, antioxidants and functional foods: Impact on human health. Pharmacognosy reviews 4: 118-26

Logsdon AF, Turner RC, Lucke-Wold BP, Robson MJ, Naser ZJ, et al. 2014. Altering endoplasmic reticulum stress in a model of blast-induced traumatic brain injury controls cellular fate and ameliorates neuropsychiatric symptoms. Frontiers in cellular neuroscience 8: 421

Long JB, Bentley TL, Wessner KA, Cerone C, Sweeney S, Bauman RA. 2009. Blast overpressure in rats: recreating a battlefield injury in the laboratory. Journal of neurotrauma 26: 827-40

Longhi L, Saatman KE, Fujimoto S, Raghupathi R, Meaney DF, et al. 2005. Temporal window of vulnerability to repetitive experimental concussive brain injury. Neurosurgery 56: 364-74; discussion 64-74

Lopez-Castroman J, Jaussent I, Beziat S, Guillaume S, Baca-Garcia E, et al. 2014. Increased severity of suicidal behavior in impulsive aggressive patients exposed to familial adversities. Psychological medicine: 1-10 
Lu J, Moochhala S, Kaur C, Ling E. 2000. Changes in apoptosis-related protein (p53, Bax, Bcl-2 and Fos) expression with DNA fragmentation in the central nervous system in rats after closed head injury. Neuroscience letters 290: 89-92

Lu XY, Wang HD, Xu JG, Ding K, Li T. 2014. NADPH oxidase inhibition improves neurological outcome in experimental traumatic brain injury. Neurochemistry international 69: 14-9

Lucke-Wold BP, Logsdon AF, Smith KE, Turner RC, Alkon DL, et al. 2014a. Bryostatin-1 Restores Blood Brain Barrier Integrity following Blast-Induced Traumatic Brain Injury. Molecular neurobiology

Lucke-Wold BP, Naser ZJ, Logsdon AF, Turner RC, Smith KE, et al. 2015a. Amelioration of NADPH-Mediated Stress Reduces Cell Death following Blastinduced Traumatic Brain Injury. Translational Research

In Press

Lucke-Wold BP, Nguyen L, Turner RC, Logsdon AF, Chen YW, et al. 2015b. Traumatic brain injury and epilepsy: Underlying mechanisms leading to seizure. Seizure 33: 13-23

Lucke-Wold BP, Smith KE, Nguyen L, Turner RC, Logsdon AF, et al. 2015c. Sleep disruption and the sequelae associated with traumatic brain injury. Neuroscience and biobehavioral reviews 55: 68-77

Lucke-Wold BP, Turner RC, Logsdon AF, Bailes JE, Huber JD, Rosen CL. 2014b. Linking traumatic brain injury to chronic traumatic encephalopathy: identification of potential mechanisms leading to neurofibrillary tangle development. Journal of neurotrauma 31: 1129-38

Lucke-Wold BP, Turner RC, Logsdon AF, Bailes JE, Huber JD, Rosen CL. 2014c. Linking Traumatic Brain Injury to Chronic Traumatic Encephalopathy: Identification of Potential Mechanisms Leading to Neurofibrillary Tangle Development. Journal of neurotrauma

Lucke-Wold BP, Turner RC, Logsdon AF, Nguyen L, Bailes JE, et al. 2015d. Endoplasmic reticulum stress implicated in chronic traumatic encephalopathy. Journal of neurosurgery In Press

Lucke-Wold BP, Turner RC, Logsdon AF, Simpkins JW, Alkon DL, et al. 2014d. Common Mechanisms of Alzheimer's Disease and Ischemic Stroke: The Role of Protein Kinase C in the Progression of Age-Related Neurodegeneration. Journal of Alzheimer's disease : JAD

Luo J, Nguyen A, Villeda S, Zhang H, Ding Z, et al. 2014a. Long-term cognitive impairments and pathological alterations in a mouse model of repetitive mild traumatic brain injury. Frontiers in neurology 5: 12

Luo P, Chen T, Zhao Y, Zhang L, Yang Y, et al. 2014b. Postsynaptic scaffold protein Homer 1a protects against traumatic brain injury via regulating group I metabotropic glutamate receptors. Cell death \& disease 5: e1174

Lyman M, Lloyd DG, Ji X, Vizcaychipi MP, Ma D. 2014. Neuroinflammation: the role and consequences. Neuroscience research 79: 1-12

Ma D, Jin S, Li E, Doi Y, Parajuli B, et al. 2013. The neurotoxic effect of astrocytes activated with toll-like receptor ligands. Journal of neuroimmunology 254: 10-8

Maas AI, Stocchetti N, Bullock R. 2008. Moderate and severe traumatic brain injury in adults. Lancet neurology 7: 728-41 
Macdonald CL, Johnson AM, Nelson EC, Werner NJ, Fang R, et al. 2014. Functional Status after Blast-Plus-Impact Complex Concussive Traumatic Brain Injury in Evacuated United States Military Personnel. Journal of neurotrauma

Madathil SK, Carlson SW, Brelsfoard JM, Ye P, D'Ercole AJ, Saatman KE. 2013. Astrocyte-Specific Overexpression of Insulin-Like Growth Factor-1 Protects Hippocampal Neurons and Reduces Behavioral Deficits following Traumatic Brain Injury in Mice. PloS one 8: e67204

Magnoni S, Brody DL. 2010. New perspectives on amyloid-beta dynamics after acute brain injury: moving between experimental approaches and studies in the human brain. Archives of neurology 67: 1068-73

Manley GT, Rosenthal G, Lam M, Morabito D, Yan D, et al. 2006. Controlled cortical impact in swine: pathophysiology and biomechanics. Journal of neurotrauma 23: 128-39

Mannix R, Berglass J, Berkner J, Moleus P, Qiu J, et al. 2014. Chronic gliosis and behavioral deficits in mice following repetitive mild traumatic brain injury. Journal of neurosurgery: 1-9

Mannix R, Meehan WP, Mandeville J, Grant PE, Gray T, et al. 2013. Clinical correlates in an experimental model of repetitive mild brain injury. Annals of neurology 74 : $65-75$

Marcelo A, Bix G. 2014. The potential role of perlecan domain V as novel therapy in vascular dementia. Metabolic brain disease

Maroon JC, Winkelman R, Bost J, Amos A, Mathyssek C, Miele V. 2015. Chronic traumatic encephalopathy in contact sports: a systematic review of all reported pathological cases. PloS one 10: e0117338

Martin L, Latypova X, Wilson CM, Magnaudeix A, Perrin ML, et al. 2013. Tau protein kinases: involvement in Alzheimer's disease. Ageing research reviews 12: 289309

Maughan PH, Scholten KJ, Schmidt RH. 2000. Recovery of water maze performance in aged versus young rats after brain injury with the impact acceleration model. Journal of neurotrauma 17: 1141-53

McCaffrey G, Davis TP. 2012. Physiology and pathophysiology of the blood-brain barrier: P-glycoprotein and occludin trafficking as therapeutic targets to optimize central nervous system drug delivery. Journal of investigative medicine : the official publication of the American Federation for Clinical Research 60: 1131-40

McCauley SR, Boake C, Levin HS, Contant CF, Song JX. 2001. Postconcussional disorder following mild to moderate traumatic brain injury: anxiety, depression, and social support as risk factors and comorbidities. J Clin Exp Neuropsychol 23: 792-808

McClure DJ, Zuckerman SL, Kutscher SJ, Gregory AJ, Solomon GS. 2014. Baseline neurocognitive testing in sports-related concussions: the importance of a prior night's sleep. The American journal of sports medicine 42: 472-8

McCrea M, Guskiewicz KM, Marshall SW, Barr W, Randolph C, et al. 2003. Acute effects and recovery time following concussion in collegiate football players: the NCAA Concussion Study. Jama 290: 2556-63 
McKee AC, Cantu RC, Nowinski CJ, Hedley-Whyte ET, Gavett BE, et al. 2009. Chronic traumatic encephalopathy in athletes: progressive tauopathy after repetitive head injury. Journal of neuropathology and experimental neurology 68: 709-35

McKee AC, Daneshvar DH, Alvarez VE, Stein TD. 2014. The neuropathology of sport. Acta neuropathologica 127: 29-51

McKee AC, Gavett BE, Stern RA, Nowinski CJ, Cantu RC, et al. 2010. TDP-43 proteinopathy and motor neuron disease in chronic traumatic encephalopathy. Journal of neuropathology and experimental neurology 69: 918-29

McKee AC, Stern RA, Nowinski CJ, Stein TD, Alvarez VE, et al. 2013. The spectrum of disease in chronic traumatic encephalopathy. Brain : a journal of neurology 136: 43-64

Medeiros R, Kitazawa M, Chabrier MA, Cheng D, Baglietto-Vargas D, et al. 2012. Calpain inhibitor A-705253 mitigates Alzheimer's disease-like pathology and cognitive decline in aged 3xTgAD mice. The American journal of pathology 181: 616-25

Meehan WP, 3rd, Zhang J, Mannix R, Whalen MJ. 2012. Increasing recovery time between injuries improves cognitive outcome after repetitive mild concussive brain injuries in mice. Neurosurgery 71: 885-91

Mehan ND, Strauss KI. 2012. Combined age- and trauma-related proteomic changes in rat neocortex: a basis for brain vulnerability. Neurobiology of aging 33: 1857-73

Meyerhoff JL, Lee JK, Rittase BW, Tsang AY, Yourick DL. 2004. Lipoic acid pretreatment attenuates ferric chloride-induced seizures in the rat. Brain research 1016: 139-44

Mez J, Stern RA, McKee AC. 2013. Chronic traumatic encephalopathy: where are we and where are we going? Current neurology and neuroscience reports 13: 407

Mills JD, Bailes JE, Sedney CL, Hutchins H, Sears B. 2011a. Omega-3 fatty acid supplementation and reduction of traumatic axonal injury in a rodent head injury model. Journal of neurosurgery 114: 77-84

Mills JD, Bailes JE, Turner RC, Dodson SC, Sakai J, Maroon JC. 2012. Anabolic steroids and head injury. Neurosurgery 70: 205-9; discussion 09-10

Mills JD, Hadley K, Bailes JE. 2011b. Dietary supplementation with the omega-3 fatty acid docosahexaenoic acid in traumatic brain injury. Neurosurgery 68: 474-81; discussion 81

Mitsis EM, Riggio S, Kostakoglu L, Dickstein DL, Machac J, et al. 2014. Tauopathy PET and amyloid PET in the diagnosis of chronic traumatic encephalopathies: studies of a retired NFL player and of a man with FTD and a severe head injury. Translational psychiatry 4: e441

Miyauchi T, Wei EP, Povlishock JT. 2014. Evidence for the therapeutic efficacy of either mild hypothermia or oxygen radical scavengers after repetitive mild traumatic brain injury. Journal of neurotrauma 31: 773-81

Mogha A, Guariglia SR, Debata PR, Wen GY, Banerjee P. 2012. Serotonin 1A receptormediated signaling through ERK and PKCalpha is essential for normal synaptogenesis in neonatal mouse hippocampus. Translational psychiatry 2: e66

Montenigro PH, Baugh CM, Daneshvar DH, Mez J, Budson AE, et al. 2014. Clinical subtypes of chronic traumatic encephalopathy: literature review and proposed 
research diagnostic criteria for traumatic encephalopathy syndrome. Alzheimer's research \& therapy 6: 68

Moreno JA, Halliday M, Molloy C, Radford H, Verity N, et al. 2013. Oral treatment targeting the unfolded protein response prevents neurodegeneration and clinical disease in prion-infected mice. Science translational medicine 5: 206ra138

Mori T, Wang X, Jung JC, Sumii T, Singhal AB, et al. 2002. Mitogen-activated protein kinase inhibition in traumatic brain injury: in vitro and in vivo effects. Journal of cerebral blood flow and metabolism : official journal of the International Society of Cerebral Blood Flow and Metabolism 22: 444-52

Morrison B, 3rd, Cater HL, Benham CD, Sundstrom LE. 2006. An in vitro model of traumatic brain injury utilising two-dimensional stretch of organotypic hippocampal slice cultures. Journal of neuroscience methods 150: 192-201

Morrow CM, Mruk D, Cheng CY, Hess RA. 2010. Claudin and occludin expression and function in the seminiferous epithelium. Philosophical transactions of the Royal Society of London. Series B, Biological sciences 365: 1679-96

Mosienko V, Bert B, Beis D, Matthes S, Fink H, et al. 2012. Exaggerated aggression and decreased anxiety in mice deficient in brain serotonin. Translational psychiatry 2: e122

Mouzon B, Chaytow H, Crynen G, Bachmeier C, Stewart J, et al. 2012. Repetitive mild traumatic brain injury in a mouse model produces learning and memory deficits accompanied by histological changes. Journal of neurotrauma 29: 2761-73

Mouzon BC, Bachmeier C, Ferro A, Ojo JO, Crynen G, et al. 2014. Chronic neuropathological and neurobehavioral changes in a repetitive mild traumatic brain injury model. Annals of neurology 75: 241-54

Mukhin AG, Ivanova SA, Allen JW, Faden AI. 1998. Mechanical injury to neuronal/glial cultures in microplates: role of NMDA receptors and $\mathrm{pH}$ in secondary neuronal cell death. Journal of neuroscience research 51: 748-58

Muoio V, Persson PB, Sendeski MM. 2014. The neurovascular unit - concept review. Acta physiologica 210: 790-8

Muscella A, Marsigliante S, Verri T, Urso L, Dimitri C, et al. 2008. PKC-epsilondependent cytosol-to-membrane translocation of pendrin in rat thyroid $\mathrm{PC} \mathrm{Cl} 3$ cells. Journal of cellular physiology 217: 103-12

Mychasiuk R, Hehar H, van Waes L, Esser MJ. 2014. Diet, age, and prior injury status differentially alter behavioral outcomes following concussion in rats. Neurobiology of disease

Mychasiuk R, Hehar H, van Waes L, Esser MJ. 2015. Diet, age, and prior injury status differentially alter behavioral outcomes following concussion in rats. Neurobiology of disease 73: 1-11

Nakagawa A, Manley GT, Gean AD, Ohtani K, Armonda R, et al. 2011. Mechanisms of primary blast-induced traumatic brain injury: insights from shock-wave research. Journal of neurotrauma 28: 1101-19

Naviaux RK. 2014. Metabolic features of the cell danger response. Mitochondrion 16: 717

Needham CE, Ritzel D, Rule GT, Wiri S, Young L. 2015. Blast Testing Issues and TBI: Experimental Models That Lead to Wrong Conclusions. Frontiers in neurology 6: 72 
Nelson TJ, Alkon DL. 2009. Neuroprotective versus tumorigenic protein kinase C activators. Trends in biochemical sciences 34: 136-45

Nemetz PN, Leibson C, Naessens JM, Beard M, Kokmen E, et al. 1999. Traumatic brain injury and time to onset of Alzheimer's disease: a population-based study. Am J Epidemiol 149: 32-40

Niesman IR, Schilling JM, Shapiro LA, Kellerhals SE, Bonds JA, et al. 2014. Traumatic brain injury enhances neuroinflammation and lesion volume in caveolin deficient mice. Journal of neuroinflammation 11: 39

Noble JM, Hesdorffer DC. 2013. Sport-related concussions: a review of epidemiology, challenges in diagnosis, and potential risk factors. Neuropsychology review 23: 273-84

Novokhatska T, Tishkin S, Dosenko V, Boldyriev A, Ivanova I, et al. 2013. Correction of vascular hypercontractility in spontaneously hypertensive rats using shRNAsinduced delta protein kinase $\mathrm{C}$ gene silencing. European journal of pharmacology 718: 401-7

O'Callaghan JP, Sriram K. 2004. Focused microwave irradiation of the brain preserves in vivo protein phosphorylation: comparison with other methods of sacrifice and analysis of multiple phosphoproteins. Journal of neuroscience methods 135: 15968

Ojo JO, Mouzon BC, Crawford F. 2015. Repetitive head trauma, chronic traumatic encephalopathy and tau: Challenges in translating from mice to men. Experimental neurology

Okubo S, Xi G, Keep RF, Muraszko KM, Hua Y. 2013. Cerebral hemorrhage, brain edema, and heme oxygenase-1 expression after experimental traumatic brain injury. Acta neurochirurgica. Supplement 118: 83-7

Olsson Y, Rinder L, Lindgren S, Stalhammar D. 1971. Studies on vascular permeability changes in experimental brain concussion. 3. A comparison between the effects of single and repeated sudden mechanical loading of the brain. Acta neuropathologica 19: 225-33

Omalu B. 2014. Chronic traumatic encephalopathy. Progress in neurological surgery 28: 38-49

Omalu B, Bailes J, Hamilton RL, Kamboh MI, Hammers J, et al. 2011a. Emerging histomorphologic phenotypes of chronic traumatic encephalopathy in American athletes. Neurosurgery 69: 173-83; discussion 83

Omalu B, Hammers JL, Bailes J, Hamilton RL, Kamboh MI, et al. 2011b. Chronic traumatic encephalopathy in an Iraqi war veteran with posttraumatic stress disorder who committed suicide. Neurosurgical focus 31: E3

Omalu BI, Bailes J, Hammers JL, Fitzsimmons RP. 2010a. Chronic traumatic encephalopathy, suicides and parasuicides in professional American athletes: the role of the forensic pathologist. The American journal of forensic medicine and pathology 31: 130-2

Omalu BI, DeKosky ST, Hamilton RL, Minster RL, Kamboh MI, et al. 2006. Chronic traumatic encephalopathy in a national football league player: part II. Neurosurgery 59: 1086-92; discussion 92-3 
Omalu BI, DeKosky ST, Minster RL, Kamboh MI, Hamilton RL, Wecht CH. 2005. Chronic traumatic encephalopathy in a National Football League player. Neurosurgery 57: 128-34; discussion 28-34

Omalu BI, Fitzsimmons RP, Hammers J, Bailes J. 2010b. Chronic traumatic encephalopathy in a professional American wrestler. Journal of forensic nursing 6: $130-6$

Omalu BI, Hamilton RL, Kamboh MI, DeKosky ST, Bailes J. 2010c. Chronic traumatic encephalopathy (CTE) in a National Football League Player: Case report and emerging medicolegal practice questions. Journal of forensic nursing 6: 40-6

Ommaya AK, Grubb RL, Jr., Naumann RA. 1971. Coup and contre-coup injury: observations on the mechanics of visible brain injuries in the rhesus monkey. Journal of neurosurgery 35: 503-16

Osada N, Kosuge Y, Kihara T, Ishige K, Ito Y. 2009. Apolipoprotein E-deficient mice are more vulnerable to ER stress after transient forebrain ischemia. Neurochemistry international 54: 403-9

Otani N, Nawashiro H, Fukui S, Nomura N, Yano A, et al. 2002. Differential activation of mitogen-activated protein kinase pathways after traumatic brain injury in the rat hippocampus. Journal of cerebral blood flow and metabolism : official journal of the International Society of Cerebral Blood Flow and Metabolism 22: 327-34

Ozbal S, Cankurt U, Tugyan K, Pekcetin C, Sisman AR, et al. 2015. The effects of alphalipoic acid on immature rats with traumatic brain injury. Biotech Histochem 90: 206-15

Padmaperuma B, Mark R, Dhillon HS, Mattson MP, Prasad MR. 1996. Alterations in brain protein kinase $C$ after experimental brain injury. Brain research 714: 19-26

Pandya JD, Nukala VN, Sullivan PG. 2013. Concentration dependent effect of calcium on brain mitochondrial bioenergetics and oxidative stress parameters. Frontiers in neuroenergetics 5: 10

Panzer MB, Matthews KA, Yu AW, Morrison B, 3rd, Meaney DF, Bass CR. 2012. A Multiscale Approach to Blast Neurotrauma Modeling: Part I - Development of Novel Test Devices for in vivo and in vitro Blast Injury Models. Frontiers in neurology 3: 46

Panzer MB, Wood GW, Bass CR. 2014a. Scaling in neurotrauma: how do we apply animal experiments to people? Experimental neurology 261: 120-6

Panzer MB, Wood GW, Bass CR. 2014b. Scaling in neurotrauma: How do we apply animal experiments to people? Experimental neurology

Park E, Gottlieb JJ, Cheung B, Shek PN, Baker AJ. 2011. A model of low-level primary blast brain trauma results in cytoskeletal proteolysis and chronic functional impairment in the absence of lung barotrauma. Journal of neurotrauma 28: 34357

Park S, Karunakaran U, Jeoung NH, Jeon JH, Lee IK. 2014. Physiological Effect and Therapeutic Application of Alpha Lipoic Acid. Current medicinal chemistry

Peng H, Li W, Seth DM, Nair AR, Francis J, Feng Y. 2013. (Pro)renin receptor mediates both angiotensin II-dependent and -independent oxidative stress in neuronal cells. PloS one 8: e58339 
Perez-Polo JR, Rea HC, Johnson KM, Parsley MA, Unabia GC, et al. 2013. Inflammatory consequences in a rodent model of mild traumatic brain injury. Journal of neurotrauma 30: 727-40

Peskind ER, Brody D, Cernak I, McKee A, Ruff RL. 2013. Military- and sports-related mild traumatic brain injury: clinical presentation, management, and long-term consequences. J Clin Psychiatry 74: 180-8; quiz 88

Petraglia AL, Plog BA, Dayawansa S, Chen M, Dashnaw ML, et al. 2014a. The Spectrum of Neuro-behavioral Sequelae Following Repetitive Mild Traumatic Brain Injury: A Novel Mouse Model of Chronic Traumatic Encephalopathy (CTE). Journal of neurotrauma

Petraglia AL, Plog BA, Dayawansa S, Chen M, Dashnaw ML, et al. 2014b. The spectrum of neurobehavioral sequelae after repetitive mild traumatic brain injury: a novel mouse model of chronic traumatic encephalopathy. Journal of neurotrauma 31: 1211-24

Petraglia AL, Plog BA, Dayawansa S, Dashnaw ML, Czerniecka K, et al. 2014c. The pathophysiology underlying repetitive mild traumatic brain injury in a novel mouse model of chronic traumatic encephalopathy. Surgical neurology international 5: 184

Placido AI, Oliveira CR, Moreira PI, Pereira CM. 2014. Enhanced Amyloidogenic Processing of Amyloid Precursor Protein and Cell Death Under Prolonged Endoplasmic Reticulum Stress in Brain Endothelial Cells. Molecular neurobiology

Plassman BL, Havlik RJ, Steffens DC, Helms MJ, Newman TN, et al. 2000. Documented head injury in early adulthood and risk of Alzheimer's disease and other dementias. Neurology 55: 1158-66

Plattner F, Angelo M, Giese KP. 2006. The roles of cyclin-dependent kinase 5 and glycogen synthase kinase 3 in tau hyperphosphorylation. The Journal of biological chemistry 281: 25457-65

Ponsford J, McLaren A, Schonberger M, Burke R, Rudzki D, et al. 2011. The association between apolipoprotein $\mathrm{E}$ and traumatic brain injury severity and functional outcome in a rehabilitation sample. Journal of neurotrauma 28: 1683-92

Pooler AM, Phillips EC, Lau DH, Noble W, Hanger DP. 2013. Physiological release of endogenous tau is stimulated by neuronal activity. EMBO reports 14: 389-94

Prins ML, Hales A, Reger M, Giza CC, Hovda DA. 2010. Repeat traumatic brain injury in the juvenile rat is associated with increased axonal injury and cognitive impairments. Dev Neurosci 32: 510-8

Puvenna V, Brennan C, Shaw G, Yang C, Marchi N, et al. 2014. Significance of ubiquitin carboxy-terminal hydrolase 11 elevations in athletes after sub-concussive head hits. PloS one 9: e96296

Qureshi GA, Baig S, Sarwar M, Parvez SH. 2004. Neurotoxicity, oxidative stress and cerebrovascular disorders. Neurotoxicology 25: 121-38

Raghupathi R, Mehr MF, Helfaer MA, Margulies SS. 2004. Traumatic axonal injury is exacerbated following repetitive closed head injury in the neonatal pig. Journal of neurotrauma 21: 307-16

Readnower RD, Chavko M, Adeeb S, Conroy MD, Pauly JR, et al. 2010. Increase in blood-brain barrier permeability, oxidative stress, and activated microglia in a rat 
model of blast-induced traumatic brain injury. Journal of neuroscience research 88: $3530-9$

Reljanovic M, Reichel G, Rett K, Lobisch M, Schuette K, et al. 1999. Treatment of diabetic polyneuropathy with the antioxidant thioctic acid (alpha-lipoic acid): a two year multicenter randomized double-blind placebo-controlled trial (ALADIN II). Alpha Lipoic Acid in Diabetic Neuropathy. Free radical research 31: 171-9

Reyes JF, Reynolds MR, Horowitz PM, Fu Y, Guillozet-Bongaarts AL, et al. 2008. A possible link between astrocyte activation and tau nitration in Alzheimer's disease. Neurobiology of disease 31: 198-208

Robson MJ, Turner RC, Naser ZJ, McCurdy CR, O'Callaghan JP, et al. 2014. SN79, a sigma receptor antagonist, attenuates methamphetamine-induced astrogliosis through a blockade of OSMR/gp130 signaling and STAT3 phosphorylation. Experimental neurology 254: 180-9

Rocamonde B, Paradells S, Barcia C, Garcia Esparza A, Soria JM. 2013. Lipoic acid treatment after brain injury: study of the glial reaction. Clinical \& developmental immunology 2013: 521939

Rocamonde B, Paradells S, Barcia JM, Barcia C, Garcia Verdugo JM, et al. 2012. Neuroprotection of lipoic acid treatment promotes angiogenesis and reduces the glial scar formation after brain injury. Neuroscience 224: 102-15

Roh DH, Yoon SY, Seo HS, Kang SY, Moon JY, et al. 2010. Sigma-1 receptor-induced increase in murine spinal NR1 phosphorylation is mediated by the PKCalpha and epsilon, but not the PKCzeta, isoforms. Neuroscience letters 477: 95-9

Ron D, Harding HP. 2012. Protein-folding homeostasis in the endoplasmic reticulum and nutritional regulation. Cold Spring Harbor perspectives in biology 4

Rossi G, Bastone A, Piccoli E, Morbin M, Mazzoleni G, et al. 2014. Different mutations at V363 MAPT codon are associated with atypical clinical phenotypes and show unusual structural and functional features. Neurobiology of aging 35: 408-17

Roth TL, Nayak D, Atanasijevic T, Koretsky AP, Latour LL, McGavern DB. 2014. Transcranial amelioration of inflammation and cell death after brain injury. Nature 505: 223-8

Rubenstein R, Chang B, Davies P, Wagner AMD, Robertson CS, Wang KK. 2014. A Novel, Ultrasensitive Assay for Tau: Potential for Assessing Traumatic Brain Injury in Tissues and Biofluids. Journal of neurotrauma

Rubovitch V, Shachar A, Werner H, Pick CG. 2011. Does IGF-1 administration after a mild traumatic brain injury in mice activate the adaptive arm of ER stress? Neurochemistry international 58: 443-6

Ryan NP, Catroppa C, Cooper JM, Beare R, Ditchfield M, et al. 2014. Relationships between acute imaging biomarkers and theory of mind impairment in post-acute pediatric traumatic brain injury: A prospective analysis using susceptibility weighted imaging (SWI). Neuropsychologia 66C: 32-38

Sabirzhanov B, Zhao Z, Stoica BA, Loane DJ, Wu J, et al. 2014. Downregulation of miR23a and miR-27a following experimental traumatic brain injury induces neuronal cell death through activation of proapoptotic Bcl-2 proteins. The Journal of neuroscience : the official journal of the Society for Neuroscience 34: 10055-71 
Sadik G, Tanaka T, Kato K, Yamamori H, Nessa BN, et al. 2009a. Phosphorylation of tau at Ser214 mediates its interaction with 14-3-3 protein: implications for the mechanism of tau aggregation. Journal of neurochemistry 108: 33-43

Sadik G, Tanaka T, Kato K, Yanagi K, Kudo T, Takeda M. 2009b. Differential interaction and aggregation of 3-repeat and 4-repeat tau isoforms with 14-3-3zeta protein. Biochemical and biophysical research communications 383: 37-41

Salazar AM, Grafman J. 2015. Post-traumatic epilepsy: clinical clues to pathogenesis and paths to prevention. Handbook of clinical neurology 128: 525-38

Sato T, Tsuboi K, Nomura M, Iwata M, Abe S, et al. 2014. Traumatic basal subarachnoid hemorrhage suspected to have been caused by contrecoup cerebellar contusions: a case report. Legal medicine 16: 92-4

Saulle M, Greenwald BD. 2012. Chronic traumatic encephalopathy: a review. Rehabilitation research and practice 2012: 816069

Schneider EB, Sur S, Raymont V, Duckworth J, Kowalski RG, et al. 2014. Functional recovery after moderate/severe traumatic brain injury: A role for cognitive reserve? Neurology 82: 1636-42

Shao B, Bayraktutan U. 2014. Hyperglycaemia promotes human brain microvascular endothelial cell apoptosis via induction of protein kinase C-ssI and prooxidant enzyme NADPH oxidase. Redox biology 2: 694-701

Shapira M, Licht A, Milman A, Pick CG, Shohami E, Eldar-Finkelman H. 2007. Role of glycogen synthase kinase-3beta in early depressive behavior induced by mild traumatic brain injury. Molecular and cellular neurosciences 34: 571-7

Sharma P, Su YA, Barry ES, Grunberg NE, Lei Z. 2012. Mitochondrial targeted neuron focused genes in hippocampus of rats with traumatic brain injury. International journal of critical illness and injury science 2: 172-9

Shenton ME, Hamoda HM, Schneiderman JS, Bouix S, Pasternak O, et al. 2012. A review of magnetic resonance imaging and diffusion tensor imaging findings in mild traumatic brain injury. Brain imaging and behavior 6: 137-92

Shitaka Y, Tran HT, Bennett RE, Sanchez L, Levy MA, et al. 2011. Repetitive closedskull traumatic brain injury in mice causes persistent multifocal axonal injury and microglial reactivity. Journal of neuropathology and experimental neurology 70: 551-67

Shoulders MD, Ryno LM, Genereux JC, Moresco JJ, Tu PG, et al. 2013. Stressindependent activation of XBP1s and/or ATF6 reveals three functionally diverse ER proteostasis environments. Cell reports 3: 1279-92

Sieg F, Wahle P, Pape HC. 1999. Cellular reactivity to mechanical axonal injury in an organotypic in vitro model of neurotrauma. Journal of neurotrauma 16: 1197-213

Simard JMMDPD, Pampori A, Keledjian K, Tosun C, Schwartzbauer G, et al. 2014. Exposure of the thorax to a sublethal blast wave causes a hydrodynamic pulse that leads to perivenular inflammation in the brain. Journal of neurotrauma

Simpkins JW, Green PS, Gridley KE, Singh M, de Fiebre NC, Rajakumar G. 1997. Role of estrogen replacement therapy in memory enhancement and the prevention of neuronal loss associated with Alzheimer's disease. The American journal of medicine 103: 19S-25S 
Sirko S, Behrendt G, Johansson PA, Tripathi P, Costa M, et al. 2013. Reactive glia in the injured brain acquire stem cell properties in response to sonic hedgehog. [corrected]. Cell Stem Cell 12: 426-39

Sivanandam TM, Thakur MK. 2012. Traumatic brain injury: a risk factor for Alzheimer's disease. Neuroscience and biobehavioral reviews 36: 1376-81

Slemmer JE, Weber JT. 2005. The extent of damage following repeated injury to cultured hippocampal cells is dependent on the severity of insult and inter-injury interval. Neurobiology of disease 18: 421-31

Slemmer JE, Weber JT, De Zeeuw CI. 2004. Cell death, glial protein alterations and elevated S-100 beta release in cerebellar cell cultures following mechanically induced trauma. Neurobiology of disease 15: 563-72

Small GW, Kepe V, Siddarth P, Ercoli LM, Merrill DA, et al. 2013. PET scanning of brain tau in retired national football league players: preliminary findings. The American journal of geriatric psychiatry : official journal of the American Association for Geriatric Psychiatry 21: 138-44

Smith C. 2013. Review: the long-term consequences of microglial activation following acute traumatic brain injury. Neuropathology and applied neurobiology 39: 35-44

Smith DH, Soares HD, Pierce JS, Perlman KG, Saatman KE, et al. 1995. A model of parasagittal controlled cortical impact in the mouse: cognitive and histopathologic effects. Journal of neurotrauma 12: 169-78

Smith DW, Bailes JE, Fisher JA, Robles J, Turner RC, Mills JD. 2012. Internal jugular vein compression mitigates traumatic axonal injury in a rat model by reducing the intracranial slosh effect. Neurosurgery 70: 740-6

Song J, Park KA, Lee WT, Lee JE. 2014. Apoptosis Signal Regulating Kinase 1 (ASK1): Potential as a Therapeutic Target for Alzheimer's Disease. International journal of molecular sciences 15: 2119-29

Sorce S, Krause KH. 2009. NOX enzymes in the central nervous system: from signaling to disease. Antioxidants \& redox signaling 11: 2481-504

Sosa MA, De Gasperi R, Paulino AJ, Pricop PE, Shaughness MC, et al. 2013. Blast overpressure induces shear-related injuries in the brain of rats exposed to a mild traumatic brain injury. Acta neuropathologica communications 1: 51

Spillantini MG, Iovino M, Vuono R. 2011. Release of growth factors by neuronal precursor cells as a treatment for diseases with tau pathology. Arch Ital Biol 149: 215-23

Srinivasan K, Sharma SS. 2012. 3-Bromo-7-nitroindazole attenuates brain ischemic injury in diabetic stroke via inhibition of endoplasmic reticulum stress pathway involving CHOP. Life sciences 90: 154-60

Stamatovic SM, Dimitrijevic OB, Keep RF, Andjelkovic AV. 2006. Protein kinase Calpha-RhoA cross-talk in CCL2-induced alterations in brain endothelial permeability. The Journal of biological chemistry 281: 8379-88

Steimer T, Driscoll P. 2003. Divergent stress responses and coping styles in psychogenetically selected Roman high-(RHA) and low-(RLA) avoidance rats: behavioural, neuroendocrine and developmental aspects. Stress 6: 87-100

Stein TD, Alvarez VE, McKee AC. 2014. Chronic traumatic encephalopathy: a spectrum of neuropathological changes following repetitive brain trauma in athletes and military personnel. Alzheimer's research \& therapy 6: 4 
Stern RA, Daneshvar DH, Baugh CM, Seichepine DR, Montenigro PH, et al. 2013. Clinical presentation of chronic traumatic encephalopathy. Neurology 81: 1122-9

Stern RA, Riley DO, Daneshvar DH, Nowinski CJ, Cantu RC, McKee AC. 2011. Longterm consequences of repetitive brain trauma: chronic traumatic encephalopathy. $P M \& R$ : the journal of injury, function, and rehabilitation 3: S460-7

Stutzbach LD, Xie SX, Naj AC, Albin R, Gilman S, et al. 2013. The unfolded protein response is activated in disease-affected brain regions in progressive supranuclear palsy and Alzheimer's disease. Acta neuropathologica communications 1: 31

Su E, Bell M. 2016. Diffuse Axonal Injury In Translational Research in Traumatic Brain Injury, ed. D Laskowitz, G Grant. Boca Raton (FL)

Sun MK, Alkon DL. 2006. Bryostatin-1: pharmacology and therapeutic potential as a CNS drug. CNS drug reviews 12: 1-8

Sun MK, Alkon DL. 2013. Cerebral ischemia-induced difference in sensitivity to depression and potential therapeutics in rats. Behavioural pharmacology 24: 2228

Sun MK, Alkon DL. 2014. The "Memory Kinases": Roles of PKC Isoforms in Signal Processing and Memory Formation. Progress in molecular biology and translational science 122: 31-59

Sun MK, Hongpaisan J, Alkon DL. 2009. Postischemic PKC activation rescues retrograde and anterograde long-term memory. Proceedings of the National Academy of Sciences of the United States of America 106: 14676-80

Sun MK, Hongpaisan J, Lim CS, Alkon DL. 2014. Bryostatin-1 restores hippocampal synapses and spatial learning and memory in adult fragile $\mathrm{x}$ mice. The Journal of pharmacology and experimental therapeutics 349: 393-401

Sun MK, Hongpaisan J, Nelson TJ, Alkon DL. 2008. Poststroke neuronal rescue and synaptogenesis mediated in vivo by protein kinase $\mathrm{C}$ in adult brains. Proceedings of the National Academy of Sciences of the United States of America 105: 136205

Suzuki S, Gerhold LM, Bottner M, Rau SW, Dela Cruz C, et al. 2007. Estradiol enhances neurogenesis following ischemic stroke through estrogen receptors alpha and beta. The Journal of comparative neurology 500: 1064-75

Tajes M, Eraso-Pichot A, Rubio-Moscardo F, Guivernau B, Bosch-Morato M, et al. 2014. Methylglyoxal reduces mitochondrial potential and activates Bax and caspase-3 in neurons: Implications for Alzheimer's disease. Neuroscience letters

Takuma H, Arawaka S, Mori H. 2003. Isoforms changes of tau protein during development in various species. Brain research. Developmental brain research 142: $121-7$

Talavage TM, Nauman EA, Breedlove EL, Yoruk U, Dye AE, et al. 2014. Functionallydetected cognitive impairment in high school football players without clinicallydiagnosed concussion. Journal of neurotrauma 31: 327-38

Tan Z, Turner RC, Leon RL, Li X, Hongpaisan J, et al. 2013. Bryostatin improves survival and reduces ischemic brain injury in aged rats after acute ischemic stroke. Stroke; a journal of cerebral circulation 44: 3490-7

Tartaglia MC, Hazrati LN, Davis KD, Green RE, Wennberg R, et al. 2014. Chronic traumatic encephalopathy and other neurodegenerative proteinopathies. Frontiers in human neuroscience 8: 30 
Teichert J, Tuemmers T, Achenbach H, Preiss C, Hermann R, et al. 2005. Pharmacokinetics of alpha-lipoic acid in subjects with severe kidney damage and end-stage renal disease. J Clin Pharmacol 45: 313-28

Teng JC, Kay H, Chen Q, Adams JS, Grilli C, et al. 2008. Mechanisms related to the cardioprotective effects of protein kinase $\mathrm{C}$ epsilon (PKC epsilon) peptide activator or inhibitor in rat ischemia/reperfusion injury. Naunyn-Schmiedeberg's archives of pharmacology 378: 1-15

Thal SC, Luh C, Schaible EV, Timaru-Kast R, Hedrich J, et al. 2012. Volatile anesthetics influence blood-brain barrier integrity by modulation of tight junction protein expression in traumatic brain injury. PloS one 7: e50752

Theadom A, Parmar P, Jones K, Barker-Collo S, Starkey N, et al. 2014. Frequency and impact of recurrent traumatic brain injury in a population-based sample. Journal of neurotrauma

Timaru-Kast R, Luh C, Gotthardt P, Huang C, Schafer MK, et al. 2012. Influence of age on brain edema formation, secondary brain damage and inflammatory response after brain trauma in mice. PloS one 7: e43829

Toklu HZ, Hakan T, Biber N, Solakoglu S, Ogunc AV, Sener G. 2009. The protective effect of alpha lipoic acid against traumatic brain injury in rats. Free radical research 43: 658-67

Tompkins P, Tesiram Y, Lerner M, Gonzalez LP, Lightfoot S, et al. 2013. Brain injury: neuro-inflammation, cognitive deficit, and magnetic resonance imaging in a model of blast induced traumatic brain injury. Journal of neurotrauma 30: 188897

Tran HT, LaFerla FM, Holtzman DM, Brody DL. 2011. Controlled cortical impact traumatic brain injury in 3xTg-AD mice causes acute intra-axonal amyloid-beta accumulation and independently accelerates the development of tau abnormalities. The Journal of neuroscience : the official journal of the Society for Neuroscience 31: 9513-25

Tran HT, Sanchez L, Brody DL. 2012. Inhibition of JNK by a peptide inhibitor reduces traumatic brain injury-induced tauopathy in transgenic mice. Journal of neuropathology and experimental neurology 71: 116-29

Tsujio I, Tanaka T, Kudo T, Nishikawa T, Shinozaki K, et al. 2000. Inactivation of glycogen synthase kinase-3 by protein kinase $\mathrm{C}$ delta: implications for regulation of tau phosphorylation. FEBS Lett 469: 111-7

Turner RC, Lucke-Wold BP, Logsdon AF, Robson MJ, Dashnaw ML, et al. 2015. The Quest to Model Chronic Traumatic Encephalopathy: A Multiple Model and Injury Paradigm Experience. Frontiers in neurology 6: 222

Turner RC, Lucke-Wold BP, Robson MJ, Omalu BI, Petraglia AL, Bailes JE. 2012a. Repetitive traumatic brain injury and development of chronic traumatic encephalopathy: a potential role for biomarkers in diagnosis, prognosis, and treatment? Frontiers in neurology 3: 186

Turner RC, Naser ZJ, Bailes JE, Smith DW, Fisher JA, Rosen CL. 2012b. Effect of slosh mitigation on histologic markers of traumatic brain injury: laboratory investigation. Journal of neurosurgery 117: 1110-8 
Turner RC, Naser ZJ, Logsdon AF, DiPasquale KH, Jackson GJ, et al. 2013. Modeling clinically relevant blast parameters based on scaling principles produces functional \& histological deficits in rats. Experimental neurology 248: 520-9

Turner RC, Seminerio MJ, Naser ZJ, Ford JN, Martin SJ, et al. 2012c. Effects of aging on behavioral assessment performance: implications for clinically relevant models of neurological disease. Journal of neurosurgery 117: 629-37

Turner RC, VanGilder RL, Naser ZJ, Lucke-Wold BP, Bailes JE, et al. 2014. Elucidating the severity of preclinical traumatic brain injury models: a role for functional assessment? Neurosurgery 74: 382-94; discussion 94

Uranga RM, Giusto NM, Salvador GA. 2009. Iron-induced oxidative injury differentially regulates PI3K/Akt/GSK3beta pathway in synaptic endings from adult and aged rats. Toxicological sciences : an official journal of the Society of Toxicology 111: 331-44

Uryu K, Laurer H, McIntosh T, Pratico D, Martinez D, et al. 2002. Repetitive mild brain trauma accelerates Abeta deposition, lipid peroxidation, and cognitive impairment in a transgenic mouse model of Alzheimer amyloidosis. The Journal of neuroscience : the official journal of the Society for Neuroscience 22: 446-54

Van Den Heuvel C, Thornton E, Vink R. 2007. Traumatic brain injury and Alzheimer's disease: a review. Progress in brain research 161: 303-16

Wada H, Shintani D, Ohlrogge J. 1997. Why do mitochondria synthesize fatty acids? Evidence for involvement in lipoic acid production. Proceedings of the National Academy of Sciences of the United States of America 94: 1591-6

Wagener FA, Dankers AC, van Summeren F, Scharstuhl A, van den Heuvel JJ, et al. 2013. Heme Oxygenase-1 and breast cancer resistance protein protect against heme-induced toxicity. Current pharmaceutical design 19: 2698-707

Wagner AK, Bayir H, Ren D, Puccio A, Zafonte RD, Kochanek PM. 2004a. Relationships between cerebrospinal fluid markers of excitotoxicity, ischemia, and oxidative damage after severe TBI: the impact of gender, age, and hypothermia. Journal of neurotrauma 21: 125-36

Wagner AK, Willard LA, Kline AE, Wenger MK, Bolinger BD, et al. 2004b. Evaluation of estrous cycle stage and gender on behavioral outcome after experimental traumatic brain injury. Brain research 998: 113-21

Wagner AK, Zitelli KT. 2013. A Rehabilomics focused perspective on molecular mechanisms underlying neurological injury, complications, and recovery after severe TBI. Pathophysiology : the official journal of the International Society for Pathophysiology / ISP 20: 39-48

Walf AA, Frye CA. 2007. The use of the elevated plus maze as an assay of anxietyrelated behavior in rodents. Nature protocols 2: 322-8

Wali B, Sayeed I, Stein DG. 2011. Improved behavioral outcomes after progesterone administration in aged male rats with traumatic brain injury. Restorative neurology and neuroscience 29: 61-71

Walker KR, Tesco G. 2013. Molecular mechanisms of cognitive dysfunction following traumatic brain injury. Frontiers in aging neuroscience 5: 29

Wang G, Jiang X, Pu H, Zhang W, An C, et al. 2013a. Scriptaid, a novel histone deacetylase inhibitor, protects against traumatic brain injury via modulation of PTEN and AKT pathway : scriptaid protects against TBI via AKT. 
Neurotherapeutics : the journal of the American Society for Experimental NeuroTherapeutics 10: 124-42

Wang G, Shi Y, Jiang X, Leak RK, Hu X, et al. 2015. HDAC inhibition prevents white matter injury by modulating microglia/macrophage polarization through the GSK3beta/PTEN/Akt axis. Proceedings of the National Academy of Sciences of the United States of America 112: 2853-8

Wang JZ, Xia YY, Grundke-Iqbal I, Iqbal K. 2013b. Abnormal hyperphosphorylation of tau: sites, regulation, and molecular mechanism of neurofibrillary degeneration. Journal of Alzheimer's disease : JAD 33 Suppl 1: S123-39

Wang T, Huang Y, Zhang M, Wang L, Wang Y, et al. 2013c. [Gly14]-Humanin offers neuroprotection through glycogen synthase kinase-3beta inhibition in a mouse model of intracerebral hemorrhage. Behavioural brain research 247: 132-9

Wang W, Wang W, Mei X, Huang J, Wei Y, et al. 2009. Crosstalk between spinal astrocytes and neurons in nerve injury-induced neuropathic pain. PloS one 4: e6973

Wang Y, Arun P, Wei Y, Oguntayo S, Gharavi R, et al. 2014. Repeated blast exposures cause brain DNA fragmentation in mice. Journal of neurotrauma 31: 498-504

Wang ZF, Yin J, Zhang Y, Zhu LQ, Tian Q, et al. 2010. Overexpression of tau proteins antagonizes amyloid-beta-potentiated apoptosis through mitochondria-caspase-3 pathway in N2a cells. Journal of Alzheimer's disease : JAD 20: 145-57

Wasserman L, Shaw T, Vu M, Ko C, Bollegala D, Bhalerao S. 2008. An overview of traumatic brain injury and suicide. Brain injury : [BI] 22: 811-9

Watanabe T, Elovic E, Zafonte R. 2010. Chronic traumatic encephalopathy. $P M \& R$ : the journal of injury, function, and rehabilitation 2: 671-5

Weber JT. 2007. Experimental models of repetitive brain injuries. Progress in brain research 161: 253-61

Wei W, Wang H, Wu Y, Ding K, Li T, et al. 2015. Alpha lipoic acid inhibits neural apoptosis via a mitochondrial pathway in rats following traumatic brain injury. Neurochemistry international

Weil ZM, Gaier KR, Karelina K. 2014. Injury timing alters metabolic, inflammatory and functional outcomes following repeated mild traumatic brain injury. Neurobiology of disease 70: 108-16

Weiss JM, Maruszewski JP, Smith WA. 1999. Implicit solution of preconditioned Navier-Stokes equations using algebraic multigrid. Aiaa J 37: 29-36

Whittington RA, Bretteville A, Dickler MF, Planel E. 2013. Anesthesia and tau pathology. Prog Neuropsychopharmacol Biol Psychiatry 47: 147-55

Wu A, Ying Z, Gomez-Pinilla F. 2004. Dietary omega-3 fatty acids normalize BDNF levels, reduce oxidative damage, and counteract learning disability after traumatic brain injury in rats. Journal of neurotrauma 21: 1457-67

Wu J, Pajoohesh-Ganji A, Stoica BA, Dinizo M, Guanciale K, Faden AI. 2012. Delayed expression of cell cycle proteins contributes to astroglial scar formation and chronic inflammation after rat spinal cord contusion. Journal of neuroinflammation 9: 169

Wu J, Zhao Z, Sabirzhanov B, Stoica BA, Kumar A, et al. 2014. Spinal cord injury causes brain inflammation associated with cognitive and affective changes: role of 
cell cycle pathways. The Journal of neuroscience : the official journal of the Society for Neuroscience 34: 10989-1006

Wu X, Mao H, Liu J, Xu J, Cao J, et al. 2013. Dynamic change of SGK expression and its role in neuron apoptosis after traumatic brain injury. Int J Clin Exp Pathol 6: 1282-93

Xia YP, He QW, Li YN, Chen SC, Huang M, et al. 2013. Recombinant human sonic hedgehog protein regulates the expression of $\mathrm{ZO}-1$ and occludin by activating angiopoietin-1 in stroke damage. PloS one 8: e68891

Xu C, Liu QY, Alkon DL. 2014. PKC activators enhance GABAergic neurotransmission and paired-pulse facilitation in hippocampal CA1 pyramidal neurons. Neuroscience 268: 75-86

Xu S, Zhou X, Yuan D, Xu Y, He P. 2013. Caveolin-1 scaffolding domain promotes leukocyte adhesion by reduced basal endothelial nitric oxide-mediated ICAM-1 phosphorylation in rat mesenteric venules. American journal of physiology. Heart and circulatory physiology 305: $\mathrm{H} 1484-93$

Yamashita T, Hideyama T, Hachiga K, Teramoto S, Takano J, et al. 2012. A role for calpain-dependent cleavage of TDP-43 in amyotrophic lateral sclerosis pathology. Nat Commun 3: 1307

Yang CC, Kuai XX, Li YL, Zhang L, Yu JC, et al. 2013. Cornel Iridoid Glycoside Attenuates Tau Hyperphosphorylation by Inhibition of PP2A Demethylation. Evidence-based complementary and alternative medicine : eCAM 2013: 108486

Yang K, Taft WC, Dixon CE, Todaro CA, Yu RK, Hayes RL. 1993. Alterations of protein kinase $\mathrm{C}$ in rat hippocampus following traumatic brain injury. Journal of neurotrauma 10: 287-95

Yang K, Taft WC, Dixon CE, Yu RK, Hayes RL. 1994. Endogenous phosphorylation of a 61,000 dalton hippocampal protein increases following traumatic brain injury. Journal of neurotrauma 11: 523-32

Yang L, Shah KK, Abbruscato TJ. 2012. An in vitro model of ischemic stroke. Methods in molecular biology 814: 451-66

Ye J, Rawson RB, Komuro R, Chen X, Dave UP, et al. 2000. ER stress induces cleavage of membrane-bound ATF6 by the same proteases that process SREBPs. Molecular cell 6: 1355-64

Yen LF, Wei VC, Kuo EY, Lai TW. 2013. Distinct patterns of cerebral extravasation by Evans blue and sodium fluorescein in rats. PloS one 8: e68595

Yeoh S, Bell ED, Monson KL. 2013. Distribution of blood-brain barrier disruption in primary blast injury. Annals of biomedical engineering 41: 2206-14

Yokobori S, Mazzeo AT, Hosein K, Gajavelli S, Dietrich WD, Bullock MR. 2013. Preconditioning for traumatic brain injury. Translational stroke research 4: 25-39

Yoo J, Nichols A, Mammen J, Calvo I, Song JC, et al. 2003. Bryostatin-1 enhances barrier function in T84 epithelia through PKC-dependent regulation of tight junction proteins. American journal of physiology. Cell physiology 285: C300-9

Yoshiyama Y, Uryu K, Higuchi M, Longhi L, Hoover R, et al. 2005. Enhanced neurofibrillary tangle formation, cerebral atrophy, and cognitive deficits induced by repetitive mild brain injury in a transgenic tauopathy mouse model. Journal of neurotrauma 22: 1134-41 
Yu H, Wang P, An P, Xue Y. 2012. Recombinant human angiopoietin-1 ameliorates the expressions of ZO-1, occludin, VE-cadherin, and PKCalpha signaling after focal cerebral ischemia/reperfusion in rats. Journal of molecular neuroscience : MN 46: 236-47

Zander NE, Piehler T, Boggs ME, Banton R, Benjamin R. 2015. In vitro studies of primary explosive blast loading on neurons. Journal of neuroscience research 93 : 1353-63

Zeng KW, Ko H, Yang HO, Wang XM. 2010. Icariin attenuates beta-amyloid-induced neurotoxicity by inhibition of tau protein hyperphosphorylation in PC12 cells. Neuropharmacology 59: 542-50

Zhang J, Teng Z, Song Y, Hu M, Chen C. 2015. Inhibition of monoacylglycerol lipase prevents chronic traumatic encephalopathy-like neuropathology in a mouse model of repetitive mild closed head injury. Journal of cerebral blood flow and metabolism : official journal of the International Society of Cerebral Blood Flow and Metabolism 35: 706

Zhang QG, Laird MD, Han D, Nguyen K, Scott E, et al. 2012. Critical role of NADPH oxidase in neuronal oxidative damage and microglia activation following traumatic brain injury. PloS one 7: e34504

Zhang R, Harding P, Garvin JL, Juncos R, Peterson E, et al. 2009. Isoforms and functions of NAD(P)H oxidase at the macula densa. Hypertension 53: 556-63

Zhao Y, Luo P, Guo Q, Li S, Zhang L, et al. 2012. Interactions between SIRT1 and MAPK/ERK regulate neuronal apoptosis induced by traumatic brain injury in vitro and in vivo. Experimental neurology 237: 489-98

Zhou S, Yin X, Zheng Y, Miao X, Feng W, et al. 2014. Metallothionein prevents intermittent hypoxia-induced cardiac endoplasmic reticulum stress and cell death likely via activation of Akt signaling pathway in mice. Toxicology letters

Zhu X, Zhang J, Sun H, Jiang C, Dong Y, et al. 2014. Ubiquitination of inositol-requiring enzyme 1 (IRE1) by the E3 ligase CHIP mediates the IRE1/TRAF2/JNK pathway. The Journal of biological chemistry

Zohar O, Lavy R, Zi X, Nelson TJ, Hongpaisan J, et al. 2011. PKC activator therapeutic for mild traumatic brain injury in mice. Neurobiology of disease 41: 329-37 


\title{
Curriculum Vitae
}

\author{
Brandon P. Lucke-Wold
}

Phone: (719) 459-2760

Email: Bwold@mix.wvu.edu

Home Address:

548 Morgan St.

Morgantown, WV 26505

Research Interest: Traumatic brain injury and ischemic stroke

\section{Education:}

- College: Baylor University. Major: Neuroscience. Minor: Chemistry. GPA: 3.86 (2007-2011) Graduated Magna Cum Laude

- MD/PhD Program and Global Health Track: West Virginia University Health Science Center. GPA: 4.0 (2011-Present)

- Master's Clinical and Translational Science: West Virginia University Health Science Center. GPA: 4.0 (2015-Present)

\section{College Programs:}

- Baylor Honors Program

- Baylor Interdisciplinary Core

- Fellow-The Academy of Leadership and Civic Engagement

\section{Publications:}

\section{Books/Book Chapters:}

1. Lucke-Wold, B.P. (2011) Embarking on New Paths in the World of Metallacarborane Chemistry. Germany: Lambert Academic Publishing. 1-80.

2. Turner, R.C., Lucke-Wold, B.P., Miller, D.B., O'Collaghan, J.P., Rosen, C.L., and Huber, J.D. (2012) Neuropoietic Cytokines and Neural Injury: Alterations in 
JAK2/STAT3 Signaling Associated with Aging. Neurological Disorders: New Research. Nova Publishers. 27-48.

3. Turner, R.C., Lucke-Wold, B.P., Tan, Z., Rosen, C.L., and Huber, J.D. (2012) Modulation of Protein Kinase C Isoforms: a Potential Therapeutic for Ischemic Stroke? Ischemic Stroke: Symptoms, Prevention and Recovery. Nova Publishers. 171-190.

4. Lucke-Wold, B.P., Logsdon, A.F., Turner, R.C., Rosen, C.L., and Huber, J.D. (2014) Aging, the Metabolic Syndrome, and Ischemic Stroke: Redefining the Approach for Studying the Blood Brain Barrier in a Complex Neurological Disease. Pharmacology and the Blood Brain Barrier: Targeting CNS Disorders. Advances in Pharmacology. 71:411-49.

5. Nguyen, L. Lucke-Wold, B.P., Mookerjee, S., Kaushal, N., and Matsumoto, R.R. (2016) Sigma-1 receptors and neurodegenerative diseases: Towards a hypothesis of sigma-1 receptors as amplifiers of neurodegeneration and neuroprotection. Advances in Experimental Medicine and Biology. Springer. In press

6. Logsdon, A.F., Lucke-Wold, B.P., Rosen, C.L., and Huber, J.D. (2016) Aging and Cerebrovascular Dysfunction. Primer on Cerebrovascular Diseases. Academic Press. In press

Accepted Journal Articles:

1. Lucke-Wold, B.P. (2011). Where to Draw the Line: the Balance Between Research and Medicine. The International Undergraduate Journal for Service Learning, Leadership, and Social Change. 1:11-29.

2. Lucke-Wold, B.P. (2011). The Varied Uses of Conditioned Place Preference in Behavioral Neuroscience Research: An Investigation of Alcohol Administration in Model Organisms. Impulse: Premier Undergraduate Journal for Neuroscience Research. 11:1-9.

3. Turner, R.C., Lucke-Wold, B.P., Robson, M.J., Omalu, B.I., Petraglia, A.L., and Bailes, J.E. (2012) Repetitive Traumatic Brain Injury and Development of Chronic Traumatic Encephalopathy: A Potential Role for Biomarkers in Diagnosis, Prognosis, and Treatment? Frontiers in Neurology: Frontiers in Neurotrauma. 3:186

4. Lucke-Wold, B.P., Turner, R.C., Lucke-Wold, A.N., Rosen, C.L., and Huber, J.D. (2012). Age and the Metabolic Syndrome as Risk Factors for Ischemic Stroke: Improving Preclinical Models of Ischemic Stroke. Yale Journal of Biology and Medicine. 85(4):523-39.

5. Turner R.C., Lucke-Wold, B.P., Lucke-Wold, A.N., Elliott, A.S., Logsdon, A.F., Rosen, C.L., Huber, J.D. (2013) Neuroprotection for Ischemic Stroke: Moving Past Shortcomings and Identifying Promising Directions. International Journal of Molecular Science. 14(1):1890-917.

6. Turner, R.C., VanGilder, R.L., Naser, Z.J., Lucke-Wold, B.P., Bailes, J.E., Matsumoto, R.R., Huber, J.D., Rosen, C.L. (2014) Elucidating the Severity of 
Preclinical Traumatic Brain Injury Models: A Role for Functional Assessment? Neurosurgery. 74(4):382-94.

7. Lucke-Wold, B.P., Turner, R.C., Logsdon, A.F., Bailes, J.E., Huber, J.D., Rosen, C.L. (2014) Linking Traumatic Brain Injury to Chronic Traumatic Encephalopathy: Identification of Potential Mechanisms Leading to Neurofibrillary Tangle Development. Journal of Neurotrauma. 31(13):1129-38.

8. Lucke-Wold, B.P., Turner, R.C., Logsdon, A.F., Nguyen, L., DiPasquale, K., Huber, J.D., Rosen, C.L. (2014) Metabolic Syndrome and its Profound Effect on the Development of Ischemic Stroke. American Medical Student Research Journal. 1(1):29-38

9. Lucke-Wold, B.P. (2014) Finding Balance: Running Through the Journey of Medical School. American College of Physicians: Impact. 5(14):epub

10. Tan, Z., Li, X., Turner, R.C., Logsdon, A.F., Lucke-Wold, B.P., DiPasquale, K., Chen, R., Huber, J.D., Rosen, C.L. (2014) Combination treatment of r- tPA and apyrase reduces mortality rate and hemorrhagic transformation $6 \mathrm{~h}$ after ischemic stroke in aged female rats. European Journal of Pharmacology. 738:368-73.

11. Lucke-Wold, B.P., Turner, R.C., Logsdon, A.F., Simpkins, J.W., Alkon, D.L., Smith, K.E., Chen, Y., Tan, Z., Huber, J.D., Rosen, C.L. (2014) Common Mechanisms of Alzheimer's disease and Ischemic Stroke: the Role of Protein Kinase $\mathrm{C}$ in the Progression of Age-related Neurodegeneration. Journal of Alzheimer's Disease. 43(3):711-24.

12. Logsdon, A.F., Lucke-Wold, B.P., Rosen, C.L., Huber, J.D. (2014) Disparity among Neural Injury Models and the Unfolded Protein Response. Journal of Neurological Disorders and Stroke. 2(5):1083.

13. Lucke-Wold, B.P., Logsdon, A.F., Smith, K.E., Turner, R.C., Alkon, D.L., Tan, Z., Naser, Z.J., Knotts, C.M., Huber, J.D., Rosen C.L. (2014) Bryostatin-1 Restores Blood Brain Barrier Integrity following Blast-Induced Traumatic Brain Injury. Journal of Molecular Neurobiology. 52:1119-34

14. Logsdon, A.F., Turner, R.C., Lucke-Wold, B.P., Robson, M.J., Naser, Z.J., Smith, K.E., Matsumoto, R.R., Huber, J.D., and Rosen, C.L. (2014) Altering endoplasmic reticulum stress in a model of blast-induced traumatic brain injury controls cellular fate and ameliorates neuropsychiatric symptoms. Frontiers in Cellular Neuroscience. 8:421

15. Nguyen, L., Lucke-Wold, B.P., Mookerjee, S.A., Cavendish, J.Z., Robson, M.J., Scandinaro, A.L., Matsumoto, R.R. (2014) Role of Sigma-1 Receptors in Neurodegenerative Diseases. Journal of Pharmacological Sciences. 127(1):17-29

16. Logsdon, A.F.*, Lucke-Wold, B.P.*, Huber, J.D., Rosen, C.L., Simpkins, J.W. (2015) Role of Microvascular Disruption in Brain Damage from Traumatic Brain Injury. Comprehensive Physiology. 5(3): 1147-60

17. Bonasso, P., Lucke-Wold, B.P., Jacob, G. (2015) Osteonecrosis of interphalangeal joint of thumb two months after rattlesnake bite. Hand Surgery. 20(2): 330-332

18. Bonasso, P., Lucke-Wold, B.P., Reed, Z., Bozek, J., Cottrell, S. (2015) Investigating the Impact of Preparation Strategies on USMLE Step 1 Performance. MedEdPublish. 4:5 
19. Lucke-Wold, B.P., Turner, R.C., Logsdon, A.F., Nguyen, L., Bailes, J.E., Lee, J.M., Robson, M.J., Omalu, B.I., Smith, K.E., Huber, J.D., Rosen, C.L. (2015) Endoplasmic Reticulum Stress Implicated in Chronic Traumatic Encephalopathy. Journal of Neurosurgery. 18: 1-16

20. Lucke-Wold, B.P., Smith, K.E., Nguyen, L., Turner, R.C., Logsdon, A.F., Jackson, G.J., Huber, J.D., Rosen, C.L., Miller, D.B. (2015) Sleep Disruption and the Sequelae Associated with Traumatic Brain Injury. Neuroscience and Biobehavioral Reviews. 55: 68-77

21. Lucke-Wold, B.P., Bonasso, P.C., Turner, R., Cassim, R. (2015) Adenocarcinoma of the Cecum with Rare Splenic Metastasis. West Virginia Medical Journal. Accepted

22. Tan, Z.*, Lucke-Wold, B.P.*, Logsdon, A.F., Turner, R.C., Tan, C. Li, X., Hongpaison, J., Alkon, D.L., Simpkins, J.W., Rosen, C.L., Huber J.D. (2015) Bryostatin Extends tPA Time Window to 6 Hours Following Middle Cerebral Artery Occlusion in Aged Female Rats. European Journal of Pharmacology. 764:404-412

23. Bailes, J.E., Turner, R.C., Lucke-Wold, B.P., Patel, V. Lee, J.M. (2015) Chronic Traumatic Encephalopathy: Is it Real? The Relationship Between Neurotrauma and Neurodegeneration. Neurosurgery. 1:15-24

24. Lucke-Wold, B.P., Naser, Z., Logsdon, A.F., Turner, R.C., Smith, K.E., Robson, M.J., Bailes, J.E., Lee, J.M. Rosen, C.L., Huber, J.D. (2015) Amelioration of NADPH-Mediated Stress Reduces Cell Death following Blast-induced Traumatic Brain Injury. Translational Research. 166:509-528

25. Bonasso, P., Lucke-Wold, B.P., Riffon, M., Long, D., Wilson, A., Knight, J. (2015) The Effect of Sporting Events on Medical Transport Time at a Level 1 Trauma Center: a Retrospective Cohort Study. West Virginia Medical Journal. Accepted.

26. Lucke-Wold, B.P., Nguyen, L., Turner, R.C., Logsdon, A.F., Chen, Y., Smith, K.E. Huber, J.D., Matsumoto, R. Rosen, C.L., Tucker, E. Richter, E. (2015) Traumatic Brain Injury and Epilepsy: Underlying Mechanisms Leading to Seizure. Seizure: European Journal of Epilepsy. 33: 13-23

27. Turner, R.C., Lucke-Wold, B.P., Logsdon, A.F., Robson, M.J., Lee, J.M., Bailes, J.E., Dashnaw, M.L., Huber, J.D., Petraglia, A.L., Rosen, C.L. (2015) The Quest to Model Chronic Traumatic Encephalopathy: The Way Forward for Future Discovery. Frontiers in Neurotrauma. 6:222

28. Turner, R.C.*, Lucke-Wold, B.P.* , Logsdon, A.F.*, Robson, M.J., Dashnaw, M.L., Huang, J.H., Smith, K.E., Huber, J.D., Rosen, C.L., Petraglia, A.L. (2015) Modeling Chronic Traumatic Encephalopathy: A Multiple Model \& Injury Paradigm Experience. Frontiers in Neurotrauma. 6:223

29. Nguyen, L., Thomas, K.L., Lucke-Wold, B.P., Cavendish, J.Z., Crowe, M.S., Matsumoto, R.R. (2016) Dextromethorphan: An update on its utility for neurological and neuropsychiatric disorders. Pharmacology and Therapeutics. [Epub]

Current Submissions 
1. Logsdon, A. Lucke-Wold, B.P., Nguyen, L., Matsumoto, R., Turner, R.C., Rosen, C.L., Huber, J.D. (2015) Salubrinal reduces markers of neuroinflammation and improves outcome in a rodent model of traumatic brain injury. Experimental Neurology. Under Review

2. Lucke-Wold, B.P., Bonasso, P. Cassim, R. (2015) Primary Colon Adenocarcinoma and Primary Rectal Carcinoma with Metastatic Disease to the Left Axilla. American Journal of Surgery. Under Review

3. Lucke-Wold, B.P., Phillips, M., Turner, R.C., Logsdon, A.F., Smith, K.E., Huber, J.D., Rosen, C.L., Regele, J. (2015) Elucidating the Role of Compression Waves and Impact Duration for Generating mild Traumatic Brain Injury in Rats. Brain Injury. Under Review

4. Turner, R.C., Lucke-Wold, B.P., Hwang, R., Underwood, B.D. (2015) Lung Cancer Metastasis Presenting as a Solitary Skull Mass: A Case Report and Review of the Literature. Neurological Medicine. Under Review

5. Bonasso, P., Lucke-Wold, B.P., Jacob, G. (2015) Re-fracture of Distal Radius in the Setting of Trauma. Medical Student Research Journal. Under Review

6. Turner, R.C., Lucke-Wold, B.P., Robson, M.J., Lee, J.M., Lee, J.M., Bailes, J.E. (2015) Alzheimer's disease and Chronic Traumatic Encephalopathy: Distinct but Possibly Overlapping Disease Entities. Brain Injury. Under Review.

7. Katsevman, G., Turner, R., Lucke-Wold, B.P., Sedney, C., Bhatia, S. (2016) Osteopathia Striata with Cranial Sclerosis (OSCS): Review of the Literature \& Case Report Demonstrating Challenges of Posterior Cervico-thoracic Fusion after Trauma. Acta Neurochirurgica. Under Review

8. Lucke-Wold, B.P.*, Turner, R.C., Josiah, D., Bhatia, S. (2016) Effect of Age and Anticoagulation on the Natural History of Acute Convexity Subdural Hematomas. Turkish Neurosurgery. Under Review

9. Turner, R.C., Lucke-Wold, B.P., Josiah, D., Gonzalez, J., Tarabishy, A.R., Bhatia, S. (2016). Stereotactic radiosurgery planning based on time-resolved CTA for arteriovenous malformation: A case report and review of the literature. Stereotactic and Functional Neurosurgery. Under Review

10. Ciporen, J., Lucke-Wold, B.P., Mendez, G., Cameron, W. (2016). Managing cavernous carotid surgical complications endoscopically: A simulated costeffective and reproducible training opportunity. Journal of Neurosurgery. Under Review

11. Ciporen, J., Lucke-Wold, B.P., Dogan, A., Cetas, J., Cameron, W.E. (2016). Dual endoscopic endonasal transphenoidal and precaruncular transorbital approaches for clipping the cavernous carotid artery: A cadaveric simulation. Skull Base. Under Review

12. Ciporen, J.N., Lucke-Wold, B.P., Dogan, A., Cetas, J., Cameron, W. (2016). Endoscopic endonasal transclival approach vs dual transorbital port technique for clip application to the posterior circulation: A cadaveric anatomic and cerebral circulation simulation study. Skull Base. Under Review.

\section{Poster Presentations:}


1. Logsdon, A.F., Turner, R.C., Tan, Z., DiPasquale, K., Lucke-Wold, B.P., Rosen, C.L., Huber, J.D. (2012) Alterations to the JAK2/STAT3 signaling pathway may affect ER stress levels in the aged rat stroke model. WVU Center for Neuroscience Annual Retreat.

2. Tan, Z., Li, X., Turner, R.C., Logsdon, A., Lucke-Wold, B.P., DiPasquale, K., Chen, R., Rosen, C.L., Huber, J.D. (2012) Combination treatment of r-tPA and APT 102 reduces cerebral infarct volume, improves neurological deficit score and prevents hemorrhagic transformation in embolic stroke in aged female rats. WVU Center for Neuroscience Annual Retreat.

3. Lucke-Wold, B.P., Turner, R.C., DiPasquale, K., Rosen, C.L., Huber, J.D. (2012) Pressure Wave: a Membrane-Rupture Induced Model of Traumatic Brain Injury. WVU Center for Neuroscience Annual Retreat.

4. Lucke-Wold, B.P., Bonasso, P., Riffon, M., Long, D., Wilson, A., Knight, J. (2013) County Roads Take Me Home: Population Surge and its Effect on a Rural Trauma Center. American Medical Association National Meeting.

5. Lucke-Wold, B.P., Logsdon, A.F., Turner, R.C., Huber, J.D., Rosen, C.L. (2014) Linking Acute Neurotrauma to Chronic Traumatic Encephalopathy: the Role of the ER Stress Response. WVU Van Liere Research Day.

6. Logsdon, A.F., Turner, R.C., Lucke-Wold, B.P., Robson, M.J., Naser, Z.J., Tan, Z., Matsumoto, R.R., Rosen, C.L., and Huber, J.D. (2014). Altering endoplasmic reticulum stress attenuates neural injury markers following blast-induced traumatic brain injury in male rats. WVU Van Liere Research Day.

7. Lucke-Wold, B.P., Logsdon, A.F., Turner, R.C., Huber, J.D., Rosen, C.L. (2014) PERK-mediated ER Stress: Linking Acute Blast-induced Neurotrauma with Taudependent Neurodegeneration. American College of Physicians National Meeting.

8. Lucke-Wold, B.P., Logsdon, A.F., Turner, R.C., Bailes, J.E., Lee, J., Huber, J.D., Rosen, C.L. (2014) PERK-mediated ER Stress: Linking Acute Blast-induced Traumatic Brain Injury with Chronic Traumatic Encephalopathy. American Physician Scientist Association.

9. Logsdon, A.F., Hernandez, A., Tan, C., Lucke-Wold, B.P., Turner, R.C., Tan, Z., Bibb, J.A., Rosen, C.L., Huber, J.D. (2014). Calcium-dependent mechanisms resulting from blast-induced traumatic brain injury are linked to tau hyperphosphorylation. Barriers of the CNS: Gordon Research Conference.

10. Lucke-Wold, B.P., Logsdon, A.F., Turner, R.C., Bailes, J.E., Lee, J., Huber, J.D., Rosen, C.L. (2014) Connecting Acute Neurotrauma to Chronic Traumatic Encephalopathy: the Role of the ER Stress Response. National Neurotrauma Society Meeting.

11. Lucke-Wold, B.P., Logsdon, A.F., Smith, K., Turner, R.C., Alkon, D., Huber, J.D., Rosen, C.L. (2014) Bryostatin Restores Blood Brain Barrier Integrity following Blast Injury. WVU Neuroscience Retreat.

12. Lucke-Wold, B.P., Naser, Z., Logsdon, A.F., Turner, R.C., Bailes, J.E., Lee, J., Huber, J.D., Rosen, C.L. (2014) Targeting NADPH-mediated Stress to Prevent Apoptosis following Blast Traumatic Brain Injury. Appalachian Regional Cell Conference. 
13. Lucke-Wold, B.P., Naser, Z., Logsdon, A.F., Turner, R.C., Bailes, J.E., Lee, J., Huber, J.D., Rosen, C.L. (2015) Elucidating the Role of Oxidative Stress in Blast Injury. WVU Van Liere.

14. Lucke-Wold, B.P., Naser, Z., Logsdon, A.F., Turner, R.C., Bailes, J.E., Lee, J., Huber, J.D., Rosen, C.L. (2015) Reducing Oxidative Stress Following Acute Neurotrauma Improves Behavior. ASPET Experimental Biology.

15. Lucke-Wold, B.P., Logsdon A.F., Turner, R.C., Naser, Z., Bailes, J.E., Lee, J. Rosen C.L. (2015) Elucidating the Underpinnings of Neurotrauma Related Neurodegeneration ACTS: AFMR Translational Science.

16. Lucke-Wold, B.P., Logsdon A.F., Turner, R.C., Naser, Z., Bailes, J.E., Lee, J., Rosen C.L. (2015) Amelioration of NADPH-mediated Stress Reduces Cell Death following Blast Traumatic Brain Injury. Central Society of Clinical and Translational Research

17. Lucke-Wold, B.P., Logsdon A.F., Turner, R.C., Naser, Z., Bailes, J.E., Lee, J., Rosen C.L. (2015). Lipoic Acid: the Anti-Oxidant for Neurotrauma. American Society of Clinical Investigation.

18. Lucke-Wold, B.P., Logsdon A.F., Turner, R.C., Naser, Z., Bailes, J.E., Lee, J., Rosen C.L. (2015). Finding Treatments for Athletes and Soldiers Experiencing Brain Injury. American College of Physicians.

19. Lucke-Wold, B.P., Xu, S., Logsdon, A.F., Rosen, C.L., He, P. (2015) Targeting Endothelial Cell Transcellular Diapedesis: Src Dependent Activation of ICAM-1. American Association of Pharmaceutical Sciences Research Day. West Virginia University.

20. Lucke-Wold, B.P., Turner, R., Dashnaw, M., Lee, J., Rosen, C., Petraglia, A. (2015) Modeling Chronic Neurodegeneration Following Neurotrauma: A MultiModel Experience. National Neurotrauma Meeting.

21. Lucke-Wold, B.P., Logsdon, A.F., Mohammad, A., Adkins, C., Ren, X., Lockman, P., Simpkins, J., Rosen, C. (2015) Stroke and Alzheimer's Disease: Shared Vascular Mechanisms. WV Rural Health Meeting.

22. Lucke-Wold, B.P. Logsdon, A., Turner, R.C., Lockman, P., Simpkins, J., Rosen, C. (2015) Novel Approach for Concussion Treatment: Protein Kinase C and the Blood Brain Barrier. American Association of Pharmaceutical Sciences Meeting.

23. Lucke-Wold, B.P. Logsdon, A.F., Mohammad, A., Adkins, C., Ren, X., Lockman, P., Simpkins, J., Rosen, C. (2015) Understanding the Vascular Interrelationship Between Stroke and Alzheimer's Disease. Future Tox 2015 Washington, D.C.

24. Ciporen, J., Lucke-Wold, B., Mendez, G., Cua, D.J., Chen, A., Banerjee, A., Akins, P.T., Balough, B. (2016) Single Staged Resections and 3D Reconstructions of the Nasion/Glabella/Medial Orbital Wall/Frontal Sinus and Bone: Long-Term Outcome and Review of the Literature. North American Skull Base Conference.

25. Lucke-Wold, B.P., Ciporen, J., Cetas, J., Dogan, A., Cameron, W. (2016) Dual Endoscopic Endonasal Transphenoidal and Precaruncular Transorbital Approaches for Clipping of the Cavernous Carotid Artery-Enhanced Degrees of Freedom: A Cadaveric Cerebral Perfusion Simulation. North American Skull Base Conference. 
26. Lucke-Wold, B.P., Logsdon, A., Mohammad, A., Adkins, C., Ren, X., Simpkins, J., Lockman, P., Rosen, C. (2016) The Vascular Inter-relationship between Stroke and Alzheimer's disease. International Stroke Conference.

\section{Oral Presentations:}

1. Lucke-Wold, B.P. (2011) Embarking on New Paths in the World of Metallacarborane Chemistry. Thesis Defense Baylor University.

2. Lucke-Wold, B.P. (2012) Ethanol, Zebrafish, and Conditioned Place Preference. Meeting of Behavioral and Biomedical Researchers WVU.

3. Lucke-Wold, B.P., Logsdon, A.F., Turner, R.C., Tan, Z., Huber, J.D., Rosen, C.L. (2013) PERK-mediated ER Stress: Linking Acute Blast-Induced Neurotrauma with Tau-dependent Neurodegeneration. American College of Physicians WV Meeting.

4. Lucke-Wold, B.P. (2013) Blast Exposure Induces PTSD Related Traits in a Rat Model. Meeting of Behavioral and Biomedical Researchers WVU.

5. Lucke-Wold, B.P., Tan, Z., Huber, J.D., Rosen, C.L. (2013) The Effect of Bryostatin on Blood Brain Barrier Disruption and Hemorrhagic Transformation. American Association of Pharmaceutical Sciences Regional Meeting.

6. Lucke-Wold, B.P. (2014) The ER Stress Response: Linking acute traumatic brain injury to chronic tau-dependent neurodegeneration. Neurological Surgery Society of the Virginias Meeting.

7. Lucke-Wold, B.P. (2014) Linking Acute Neurotrauma to Chronic Traumatic Encephalopathy: the Role of the ER Stress Response. Van Liere

8. Lucke-Wold, B.P. (2014) The ER Stress Response: A Bridge Between Acute Neurotrauma and Chronic Neurodegeneration. Synapse Meeting.

9. Lucke-Wold, B.P. (2014) Bryostatin Restores Blood Brain Barrier Integrity following Blast-Induced Traumatic Brain Injury. WVU Neuroscience Retreat.

10. Lucke-Wold, B.P. (2014) Blast-induced Traumatic Brain Injury, Oxidative Stress, and Apoptosis: the Beneficial Effects of Alpha Lipoic Acid. American College of Physicians WV Scientific Meeting.

11. Lucke-Wold, B.P. (2014) Amelioration of NADPH-Mediated Stress Reduces Cell Death following Blast Traumatic Brain Injury. American Association of Pharmaceutical Sciences Pittsburgh Meeting.

12. Lucke-Wold, B.P. (2014) The Effects of Sporting Events on Trauma Transport Time at a Level 1 Trauma Center. Meeting of Behavioral and Biomedical Researchers WVU.

13. Lucke-Wold, B.P. (2015) Ameliorating NADPH-stress reduces cell death following blast traumatic brain injury. Neurological Surgery Society of the Virginias Meeting.

14. Lucke-Wold, B.P. (2015) The Case for Medical Marijuana in Epilepsy. West Virginia University Neuroscience Blitz.

15. Lucke-Wold, B.P. (2015) Elucidating the Underpinnings of Neurotrauma-related Neurodegeneration. ACTS:AFMR Translational Science

16. Lucke-Wold, B.P. (2015) Progressive Tauopathy: Understanding NeurotraumaRelated Neurodegeneration. Alzheimer's Association International Conference. 
17. Lucke-Wold, B.P., Logsdon, A.F., Mohammad, A., Adkins, C., Ren, X., Lockman, P., Simpkins, J., Rosen, C. (2015) Stroke and Alzheimer's Disease: Shared Vascular Mechanisms. WV ACP Scientific Meeting.

18. Lucke-Wold, B.P. (2015) Tackling Neural Injury: the Importance of Team Science. WVU Neuroscience Research Forum.

19. Lucke-Wold, B.P., Logsdon, A.F., Mohammad, A., Adkins, C. Lockman, P., Rosen, C.L. (2015) Targeting Trauma-induced Blood Brain Barrier Disruption: an Innovative Approach for Treating Concussion. Appalachian Regional Cell Conference.

20. Lucke-Wold, B.P., Ciporen, J., Cetas, J., Dogan, A., Cameron, W. (2016) Endoscopic Endonasal Transclival Approach vs Dual Transorbital Port Technique for Clip Application to the Posterior Circulation: an Anatomic and Cadaveric Cerebral Perfusion Simulation Study. North American Skull Base Society Meeting.

\section{Invited Talks}

1. Lucke-Wold, B.P. \& Turner, R.C. (2014) Modeling Blast-induced Traumatic Brain Injury \& Solving the Chronic Traumatic Encephalopathy Crisis. NorthShore Health System.

2. Lucke-Wold, B.P. \& Logsdon, A.F. (2015) Investigating Pathophysiologic Mechanisms of Traumatic Brain Injury and Neurodegeneration. NorthShore Health System.

\section{Online Forums}

1. Lucke-Wold, B.P. (2016) Novel Approach for Concussion Treatment: Protein Kinase C and the Blood Brain Barrier. AAPS Best Abstract Presentations.

\section{Funding}

- West Virginia University Research Funding and Development Grant Coinvestigator 2013: $\$ 17000$

- West Virginia University Distinguished Doctoral Fellowship 2014: \$5000 and 2015: $\$ 5000$

- American Medical Association Foundation Seed Grant 2015: \$2500

- American Foundation of Pharmaceutical Education Pre-doctoral Fellowship 2015: $\$ 10000$ and 2016: $\$ 10000$

- Neurosurgery Research and Education Foundation Medical Student Summer Research Fellowship 2015: \$2500

- Sigma Xi Grants in Aid of Research 2016: \$500

\section{Professional Meetings:}


- Neurological Surgery Society of the Virginias Meeting, Greenbrier, WV, 2014, 2015

- American Physician Scientist Association Institutional Representative Annual Meeting Chicago, IL, 2014, 2015

- National Neurotrauma Society Meeting 2014, 2015

- American College of Physicians National Meeting 2014, 2015

- Experimental Biology Meeting 2015, 2016

- American Association of Neurological Surgeons National Meeting 2015, 2016

- American Federation for Medical Research Eastern 2015, and Central 2015

- NINDS/AUPN/ANA/CNS Combining Clinical and Research Careers in Neuroscience Symposium 2015

- Alzheimer's Association International Conference 2015

- American Association of Pharmaceutical Sciences 2015

- FutureTOX 2015

- North American Skull Base Society 2016

\section{Professional Service:}

- Poster Judging - Undergraduate scholars at the WVU Neuroscience Retreat

- Nu Rho Psi National Council - Honor Society for Neuroscience

- Neuroscience Breakthrough Blitz- Speaker Committee

- Diabetes Health Coach - School of Public Health

- Blind Reviewer-Compass Clinical Trial

\section{Certification}

- $\quad$ NIH Stroke Scale $(1 / 17 / 16)$

- Modified Rankin Scale Test (1/17/16)

\section{Editorial:}

- Reviewer, Molecular Neurobiology

- Reviewer, NeuroReport

- Reviewer, PlosOne

\section{Teaching:}

- West Virginia University Graduate Neuroanatomy: Teaching Assistant and Discussion Leader (Spring 2015)

- West Virginia University Graduate Journal Club: Ran Sessions on Neuroscience Articles (Fall 2015)

- West Virginia University Introductory Psychology: Taught Lectures on Hormones and Behavior (Fall 2015) 


\section{Professional Development:}

- $\quad$ NIH Grant Writing Workshop (Fall 2015)

- NIH Ethical and Regulatory Aspects of Clinical Research (Fall 2015)

\section{Laboratory Experience:}

- Honors Thesis: Metallacarborane research under the direction of Dr. Bruce Hodson and Dr. Gordon Stone at Baylor University. My Thesis Project involved four newly synthesized metallacarborane compounds. I used simple Schlenk line procedures, nuclear magnetic imaging, spectroscopy, and chromatography tubes to execute most of the experiments I conducted. After a substantial yield of the synthesized compound had been collected, I ran the compound through a series of reactions. I also conducted a thorough examination of structure through crystallography procedures. The work was published by Lambert Academic Publishing. (Spring 2008-2011)

- Dissertation Work: Utilizing a blast traumatic brain injury model we investigated blood brain barrier disruption, axonal shearing, endoplasmic reticulum stress activation, NADPH activity, and iron mediated toxicity. Areas of interest include chronic traumatic encephalopathy, tauopathy, blast traumatic brain injury, and neurodegeneration. In addition to mechanistic investigation, we looked for therapeutic agents targeting the aforementioned pathways. We likewise investigated blood brain barrier disruption in ischemic stroke looking at the relationship between Alzheimer's disease and ischemic infarct.

- Past Laboratory Work:

1. Neuroanatomy vascular perfusions with endoscopic skull based surgery using cadaveric models. Perfusion simulation model and microfil injections to map intracranial vasculature. Oregon Health \& Science University. (Summer 2015)

2. Neuroscience research rotation focused on Alzheimer's disease and Post Traumatic Stress Disorder using rabbit eye blink conditioning under the direction of Dr. Schreurs at Blanchette Rockefeller Neurosciences Institute. (Summer 2011)

3. Neuroscience research focused on Conditioned Place Preference for alcohol under the direction of Dr. Diaz-Granados at Baylor University. Review article published in Impulse: Premier Undergraduate Journal for Neuroscience Research (Fall 2010-Spring 2011)

4. Immunology research focused on the mechanism of neddylation in specific regards to rheumatism and Alzheimer's disease. I also looked at the role of the experimental drug MLN4924 on IL-2 production in a 2B411 cell line under the direction of Dr. Dragone at National Jewish Health in Denver, CO. Presented my work in a laboratory presentation at the end of the summer. (Summer 2010) 
5. Immunology research focused on malignant melanoma and the mechanism of toll ligand receptors under the direction of Dr. Karen Newell-Rogers and Dr. Josh Cabrera at University of Colorado, Colorado Springs. (Summer 2009)

\section{Memberships:}

- Alpha Lambda Delta Honor Society (Inducted Spring 2008)

- National Society Collegiate Scholars (Inducted Spring 2008)

- Delta Epsilon Iota Honor Society (Inducted Fall 2008)

- Alpha Epsilon Delta Pre-medical Honor Society (Inducted Spring 2009)

- Golden Key Honor Society (Inducted Fall 2009)

- Nu Rho Psi- Neuroscience Honor Society (Inducted Spring 2010)

- Alpha Chi Honor Society (Inducted Spring 2010)

- Omicron Delta Kappa Circle of Leaders (Inducted Spring 2010)

- American Medical Association (Fall 2011 to present)

- American Medical Student Association (Fall 2011 to present)

- American Physician Scientist Association (Spring 2014 to present)

- American Association of Pharmaceutical Sciences (Spring 2014 to present)

- American Academy of Neurology (Spring 2014 to present)

- National Neurotrauma Society (Spring 2014 to present)

- American Society for Pharmacology and Experimental Therapeutics (Fall 2014 to present)

- American Heart Association (Spring 2015 to present)

- American Association of Neurological Surgeons (Spring 2015 to present)

- American College of Surgeons (Spring 2015 to present)

- American Chemistry Society Division of Medicinal Chemistry (Spring 2015 to present)

- Sigma Xi Research Society Full Member (Spring 2015 to present)

- American Federation of Medical Research Member (Spring 2015 to present)

- American Society of Cell Biology (Spring 2015 to present)

- Society of Toxicology (Spring 2015 to present)

- Microcirculatory Society (Fall 2015 to present)

\section{Achievements and Awards:}

\section{College}

- Congressional Award Gold Medalist (Summer 2007)

- LeadAmerica Advanced Medicine and Healthcare (Summer 2008)

- William Carey Crane Scholar (Fall 2008)

- Integrity Scholar of National Society of Collegiate Scholars (Spring 2008)

- Summer Surgery Experience Fellow-University of Cincinnati (Summer 2009)

- Intercollegiate Studies Institute-Honors Program Fellow (Summer 2009)

- Founder's Scholarship-Baylor University (Fall 2009)

- Cullen Trust Symposium on Translational Research (Spring 2010) 
- Who's Who Among American College Students (Spring 2010)

- President's Volunteer Service Award (Spring 2010)

- Scholar of Promise (Spring 2010)

- Earhart Foundation Scholarship-The Philadelphia Society (Fall 2010)

- Goodrich Foundation Scholarship-The Philadelphia Society (Spring 2011)

- Most Outstanding Neuroscience Senior (Spring 2011)

\section{MD/PhD Program}

- John E. Pescott Award for Academic Excellence (Spring 2012)

- $1^{\text {st }}$ Place Oral Presentation American College of Physicians Scientific Meeting (Fall 2013)

- $I^{\text {st }}$ Place Oral Presentation American Association of Pharmaceutical Sciences Regional Meeting (Fall 2013)

- $I^{\text {st }}$ Place Oral Presentation Neurological Surgery Society of Virginias Meeting. The Crutchfield, Gage, and Thomson Award. (Spring 2014)

- Behavioral and Biomedical Sciences Research Scholarship (Spring 2014)

- American Physician Scientist Association Travel Award (Spring 2014)

- $2^{\text {nd }}$ Place Van Liere Oral Presentation (Spring 2014)

- Synapse Travel Award (Spring 2014)

- American Association of Clinical Investigation Travel Award (Spring 2014)

- Neurotrauma Travel Award (Summer 2014)

- WVU Innovator Award Finalist (Fall 2014)

- $I^{\text {st }}$ Place Oral Presentation American College of Physicians Scientific Meeting (Fall 2014)

- $I^{\text {st }}$ Place Oral Presentation American Association of Pharmaceutical Sciences Regional Meeting (Fall 2014)

- $I^{\text {st }}$ Place Poster Presentation Appalachian Regional Cell Conference (Fall 2014)

- American Society of Pharmacology and Experimental Therapeutics Travel Award (Spring 2015)

- American Society of Pharmacology and Experimental Therapeutics Best Abstract Competition (Spring 2015)

- Central Society for Clinical and Translational Research Travel Award (Spring 2015)

- Behavioral and Biomedical Sciences Research Scholarship (Spring 2015)

- American Federation of Medical Research Scholar (Spring 2015)

- Burroughs-Wellcome Fund Trainee Travel Award Translational Science Meeting (Spring 2015)

- $1^{\text {st }}$ Place Poster Competition National American College of Physicians Meeting (Spring 2015)

- Alzheimer's Association International Conference Travel Fellowship (Summer 2015)

- Cranial Trauma Research Award American Association of Neurological Surgeons/Congress of Neurological Surgeons Section on Neurotrauma and Critical Care (Summer 2015)

- American Association of Pharmaceutical Science Biotec Graduate Student Research Award (Fall 2015)

- WV Rural Health Conference Travel Award (Fall 2015) 
- Society of Toxicology Future Tox Silver Award (Fall 2015)

- American College of Physicians Research Meeting $2^{\text {nd }}$ Place (Fall 2015)

- Washington Fellow's Program American Society of Pharmacology and Experimental Therapeutics (2015/2016)

- American Association of Neurological Surgeons Medical Student Summer Research Fellowship Award (Spring 2016)

- American Society of Pharmacology and Experimental Therapeutics Travel Award and Best Abstract Award (Spring 2016)

\section{Business:}

- Co-founder of Wright Wold Scientific, LLC. Provisional patent filed.

\section{Leadership:}

\section{College}

- Kappa Kappa Psi National Honorary Band Fraternity: Corresponding Secretary (2008-2009) and President (2009-2010). We organized and hosted the national convention while I was president.

- Baylor Neuroscience Society: Historian (2008-2009) and Vice President (20092011). We introduced students to the field of neuroscience through a speaker series and volunteer work at the local Alzheimer's care facility.

- $\quad$ Nu Rho Psi: Vice President (2010-2011) and Regional Vice President (20112014). Through this neuroscience honor society, we started an endowment to fund travel awards for students in order for students to present at national conferences.

- Delta Epsilon Iota: Treasurer (2009-2010) and President (2010-2011). Our chapter won chapter of the year, and I was awarded most outstanding chapter president for our campus projects focused on career development and networking.

- American Medical Student Association: Treasurer (2008-2010) and Vice President (2010-2011). We hosted a speaker series, organized a medical service trip, and sent members to the national convention.

- Alpha Epsilon Delta pre-med honor society: Treasurer (2009-2010). Through fundraising, we raised $\$ 3,000$ for our organizational activities.

- National Society of Collegiate Scholars: President (2009-2011) and National Leadership Council (2010-2011). We were named a gold star chapter for our focus on community service, professional development, and campus involvement.

\section{MD/PhD Program}

- Academic Council WVU School of Medicine (2011-2013). We were student advocates for our class and presented academic concerns to the professors and associate deans.

- American Medical Student Association: Vice President (2012-2013). We hosted an equipment sale for students, and hosted a forum to expose students to different specialties. 
- Students for Global Health: Vice President (2012-2013). We hosted global health week featuring several well-known keynote speakers. The week long activities increased exposure about global health concerns campus wide.

- Student Interest Group in Surgery: Committee Leader (2012-2013). We hosted a speaker's series regarding the different subspecialties of surgery and sponsored a suturing workshop.

- Neuroscience Graduate Student Organization: Committee Chair (2014-2015). I worked closely with the academic steering committee to select high quality and engaging speakers for the neuroscience seminar series. Vice President (20152016). As vice president, I helped grow the organization and expand its influence around campus.

- American Association of Pharmaceutical Sciences: Treasurer (2014-2015). I orchestrated fundraising activities and managed a several thousand-dollar budget. President (2015-2016). We sponsored a regional meeting, participated in community service endeavors, and had multiple networking events.

- American Physician Scientist Association: Partnerships Committee (2015-2016). I orchestrated new partnerships for $\mathrm{MD} / \mathrm{PhD}$ students with the American Association of Neurological Surgeons and American College of Physicians.

- American Foundation of Pharmaceutical Education: Development Committee (2016-2017). Interacted with Pharmaceutical company leaders and investors to provide sustainable funding for the AFPE pre-doctoral fellowships.

\section{Extracurricular:}

\section{College}

- Baylor University Golden Wave Marching Band (2007-2009)

- Baylor University Concert Band (2008-2010)

- Intramural Soccer and Basketball (2007-2010)

- $I^{\text {st }}$ place Great Brazos Relay long distance run (Fall 2009)

- Age-division Champion Miracle Match 5K (Fall 2008)

\section{MD/PhD Program}

- September Stride 5K (2011)

- Run For Cover 5K, Jim Dunn 5mile Run, Ruckus Pittsburgh, Weekly Yoga Classes, Racket ball, and Turkey Trot 5K (2012)

- WVU Indoor Triathlon, Run For Cover 5K, $2^{\text {nd }}$ Place Color Run, Decker's Creek Half Marathon, Ruckus, Age-division Champion Gene's run (2013).

- $2^{\text {nd }}$ Place Cooper's Rock Run, $3^{\text {rd }}$ Place Wild Warrior Challenge, State $10 \mathrm{k}$ race, Decker's Creek Half Marathon, Jim Dunn 5mile Run, Turkey Trot 5K (2014)

- Technology Park Veteran's 5k age group winner, Decker's Creek Half Marathon, Bark in the Park 5k, Akron Blue Half Marathon, Marathon on the Mountain Half Marathon (2015)

\section{Other Life Experience:}


- Worked at Angry Squirrel Tree Company (2005-2006) and Rocky Mountain Landscaping (2006-2007)

- CPR and First Aid Certified through the American Heart Association (2010-2012) Renewed (2011-2013)

- All-State Track Athlete (2007)

- Canoed from Eli, Minnesota into Canada (2004), Backpacked in the Wemuniche Wilderness of Durango, CO (2005), Snowshoed in Cooper's Rock State Park, WV (2014)

- Worked at Nuremberg YMCA in Germany during the World Cup (2006)

- Visited 48 of the 50 United States

- Zip-Lined through the jungle in Costa Rica (2010), kayaked in bioluminescent bay in Puerto Rico (2014), and took a river cruise through Hungary, Slovakia, Austria, and Germany (2016)

- Climbed Pikes Peak, Mt. Sherman, Mt. Lincoln, Mt. Democrat, and Mt. Bros in Colorado (2010)

- Honeymooned in Iceland (2011): went deep-sea fishing, hiked along waterfalls and geysers, and visited the world-renowned Blue Lagoon.

\section{Service:}

\section{College}

- $100+$ hours of volunteer work at the GI Lab of Memorial Hospital North in Colorado Springs, CO (2008-2010)

- $60+$ hours of volunteer work in Emergency Room at Hillcrest Hospital in Waco, TX (2009-2010)

- $100+$ hours mentoring under-privileged youth at Antioch Community Center (2007-2008)

- 30+ hours of volunteer work at Wesley Woods Alzheimer Care Center (20082010)

- Two medical service trips to Belize and Mexico. In Belize, we worked in Mayan villages providing medical care via a make-shift clinic, triage, and pharmacy under the direction of two United States physicians. We also gathered population data for the Red Cross. In Mexico, we worked with a local physician to provide medical care for villagers in the mountains of central Mexico. (Spring 2009 and Spring 2010 respectively)

- Civic engagement project at J.H. Hines elementary school in poverty stricken East Waco. I set up a tutoring program and parent involvement day, raised money through box tops for education, and recruited physicians to do annual check-ups for the students. (2010-2011)

\section{MD/PhD}

- 30+ hours Mushroom Leader: Mushroom is the homeless outreach at WVU School of Medicine. We pass out food, water, and clothes and do basic medical 
work under the supervision of licensed physicians. Street Rounds are every other Thursday evening. (2011-2013)

- 20+ hours volunteer work at S.A.F.E. Clinic: Student run clinic for underinsured in Morgantown and surrounding area. (2011-2014)

- Guest Presenter WVU Neuroscience Research Day: I interacted with undergraduate students, presented my research, and encouraged pursuit of further education. (Spring 2015)

- Panel Participant WVU Neuroscience Career Development Session: I served on a panel for undergraduate neuroscience students to help encourage the pursuit of medical or graduate degrees training in neuroscience. (Spring 2015)

- 100+ hours Diabetes Health Coach: taught sessions in the local community to foster diabetes prevention and enhance knowledge about diabetes. Was mentored and supervised by Dr. Ranjita Misra in the School of Public Health. (2015-2016) 